\title{
Physical and Computational Applications of Strongly-Interacting Dynamics Beyond QCD
}

Bennett , Edward, J.

How to cite:

Bennett , Edward, J. (2013) Physical and Computational Applications of Strongly-Interacting Dynamics Beyond QCD. Doctoral thesis, Swansea University.

http://cronfa.swan.ac.uk/Record/cronfa38186

Use policy:

This item is brought to you by Swansea University. Any person downloading material is agreeing to abide by the terms of the repository licence: copies of full text items may be used or reproduced in any format or medium, without prior permission for personal research or study, educational or non-commercial purposes only. The copyright for any work remains with the original author unless otherwise specified. The full-text must not be sold in any format or medium without the formal permission of the copyright holder. Permission for multiple reproductions should be obtained from the original author.

Authors are personally responsible for adhering to copyright and publisher restrictions when uploading content to the repository.

Please link to the metadata record in the Swansea University repository, Cronfa (link given in the citation reference above.)

http://www.swansea.ac.uk/library/researchsupport/ris-support/ 


\section{Physical and Computational Applications of Strongly-Interacting Dynamics Beyond QCD}

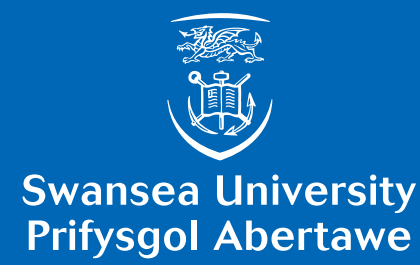

Edward Bennett

Department of Physics

Swansea University

Submitted to Swansea University in fulfilment

of the requirements for the degree of

Doctor of Philosophy

2013 


\begin{abstract}
In this thesis we investigate numerically $\mathrm{SU}(2)$ theories with Dirac - or Majorana - fermions in the adjoint representation. Majorana fermions have historically proven difficult to treat numerically; here, a change of basis is introduced that allows two Majorana fermions to be expressed in terms of one Dirac fermion. This also provides greater insight into the analysis of the properties of theories with Dirac fermions. Attention is focused on the $\mathrm{SU}(2)$ theory with a single Dirac flavour (or equivalently two Majorana flavours). Its lattice phase diagram, spectrum, and the anomalous dimension of the chiral condensate are investigated. We observe a long region of constant mass ratios and an anomalous dimension $0.9 \lesssim \gamma_{*} \lesssim 0.95$. The behaviour of the pion mass and the presence of a light scalar in particular point to behaviour that is not traditionally confining; instead the theory appears to lie in or near the conformal window. The topological susceptibility and instanton size distribution are also investigated, for the one-Dirac-flavour theory and additionally the pure-gauge and two-Dirac-flavour (Minimal Walking Technicolor) theories. The properties are found to not depend on number of flavours, indicating a quenching of the fermions in the topology, also consistent with (near-)conformal behaviour (as has previously been reported in studies of other observables for Minimal Walking Technicolor). The code used is described, and a high-performance computing benchmark developed from it is detailed. While the benchmark was originally developed to investigate the performance of different supercomputer architectures for the class of problems we are interested in. Due to the nature of the code on which it is based, it has an unusual flexibility in the demands it may place on machine's performance characteristics, which may allow it to be applicable to problems outside of lattice physics. The benchmark is used to characterise a number of machines' relative performance.
\end{abstract}




\section{Declaration}

This work has not previously been accepted in substance for any degree and is not being concurrently submitted for any degree.

Signed: (candidate)

Date:

\section{Statement 1}

This thesis is the result of my own investigations, except where otherwise stated. Where correction services have been used, the extent and nature of the correction is clearly marked in a footnote(s). Other sources are acknowledged by footnotes giving explicit references. A bibliography is appended.

Signed: (candidate)

Date:

\section{Statement 2}

I hereby give consent for my thesis, if accepted, to be available for photocopying and for inter-library loan, and for the title and summary to be made available to outside organisations.

Signed: (candidate)

Date: 


\section{Contents}

List of Figures $\quad$ v

List of Tables $\quad$ ix

1 Introduction 1

2 The Standard Model and Novel Strong Interactions 3

2.1 The Standard Model . . . . . . . . . . . . . . . . . . . . . . . 3

2.1.1 Breaking Electroweak Symmetry with a Fundamental Scalar . . 4

2.1.2 Problems with the Standard Model . . . . . . . . . . . . . . 5

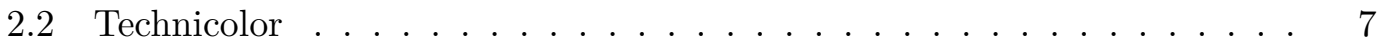

2.2.1 Beyond QCD: Phases of SU $(N)$ Gauge Theories $\ldots \ldots . . .8$

2.2 .2 Ladders and Rainbows . . . . . . . . . . . . . . . . . . . 11

2.2.3 Extended and Walking Technicolor . . . . . . . . . . . . 13

2.2 .4 Techniscalars . . . . . . . . . . . . . . . . 16

2.2 .5 Areas of research . . . . . . . . . . . . . . . . 17

2.3 Conclusions . . . . . . . . . . . . . . . . . . 18

3 Gauge Theories on the Lattice $\quad 21$

3.1 Motivation and Approach . . . . . . . . . . . . . . . 21

3.2 Algorithms . . . . . . . . . . . . . . . . . . 26

3.2 .1 RHMC algorithm . . . . . . . . . . . . . . . 26

3.2.2 Meson masses . . . . . . . . . . . . . . . . . . . . . 28

3.2.2.1 Triplet masses - the Z2SEMWall algorithm . . . . . 29

3.2.2.2 Singlet Hairpins . . . . . . . . . . . . . . . . . 32

3.2 .3 Glueballs . . . . . . . . . . . . . . . . . . . 33 
3.2.4 Anomalous Dimension . . . . . . . . . . . . . . . . . . 34

3.2.5 Wilson and Polyakov Loops . . . . . . . . . . . . . . . 36

3.2.6 Extracting Results . . . . . . . . . . . . . . . . . . . . 40

3.3 Existing Lattice Studies _ . . . . . . . . . . . . . . . . . . . . 41

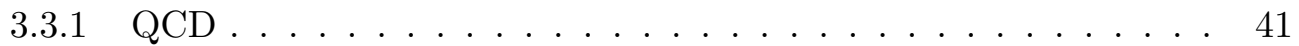

3.3 .2 Technicolor . . . . . . . . . . . . . . . . . . . 42

3.4 Theories Under Investigation . . . . . . . . . . . . . . . . 44

3.4.1 Minimal Walking Technicolor . . . . . . . . . . . . . . . 46

$3.4 .2 \quad \mathrm{SU}(2) \operatorname{Adj} \mathrm{Nf1} \ldots \ldots \ldots \ldots \ldots$

4 SU(2) with 1 Dirac (2 Majorana) Flavours 49

4.1 Methodology . . . . . . . . . . . . . . . . . . . 49

4.1 .1 Majorana decomposition . . . . . . . . . . . . . 50

$4.1 .2 \quad$ Action and $x \mathrm{SB} \ldots \ldots \ldots \ldots \ldots \ldots \ldots$

4.1 .3 Spectroscopy . . . . . . . . . . . . . . . . . . 52

4.1.4 Reexpressing spectral quantities . . . . . . . . . . . 53

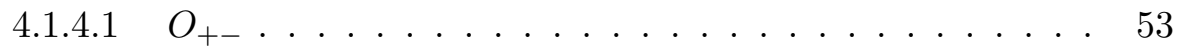

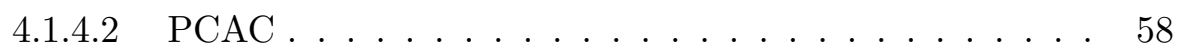

$4.1 .4 O_{++} \ldots \ldots \ldots \ldots \ldots \ldots \ldots$

4.2 Results. . . . . . . . . . . . . . . . . . . . . 60

4.2 .1 Monte Carlo . . . . . . . . . . . . . . . . . . . . . . . 61

$4.2 .2 \quad$ Spectroscopy . . . . . . . . . . . . . . . . . . . . . . 64

4.2 .3 Static potential from Wilson loops . . . . . . . . . . . . . 66

4.2.4 Anomalous dimension from the Dirac mode number . . . . . . . 69

4.3 Conclusions . . . . . . . . . . . . . . . . . . . . . . 72

5 Topology $\quad 75$

5.1 Instantons . . . . . . . . . . . . . . . . . . . . . 75

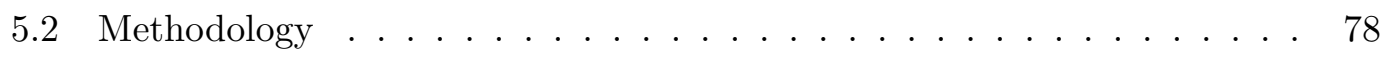

$5.2 .1 \mathrm{SU}(N)$ results . . . . . . . . . . . . . . 80

5.3 Numerical Results . . . . . . . . . . . . . . . . . . . . . . . 82

5.4 Discussion . . . . . . . . . . . . . . . . . . . . . . 91

5.5 Conclusions . . . . . . . . . . . . . . . . . . . . . 91 
6 HiRep Code and BSMBench $\quad 93$

6.1 High-Performance Computing . . . . . . . . . . . . . . . . . . 93

$6.2 \quad$ HiRep . . . . . . . . . . . . . . . . . . . . . 96

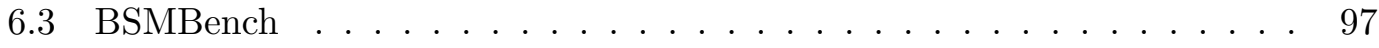

6.3.1 Why benchmark? . . . . . . . . . . . . . . 97

6.3 .2 Benchmark strategy . . . . . . . . . . . . . . . 98

6.3 .3 Tests performed . . . . . . . . . . . . . . . . 102

6.3 .4 Results . . . . . . . . . . . . . . . . . . . 104

6.3 .5 Discussion . . . . . . . . . . . . . . . . . . 104

$\begin{array}{llr}7 & \text { Conclusions } & 117\end{array}$

$\begin{array}{ll}\text { A Visualisations of Topological Charge Distributions } & 121\end{array}$

B README and LICENSE files for BSMBench $\quad 125$

B.1 README . . . . . . . . . . . . . . . . . . . 125

B.2 LICENSE . . . . . . . . . . . . . . . . . . . . . . . 129

$\begin{array}{ll}\text { References } & 131\end{array}$ 


\section{Acknowledgements}

My thanks go to all those who have helped me through this work.

Firstly to my supervisor Biagio Lucini, who has never failed to give helpful and informative advice and pointers. Thanks also to Adi Armoni, my second supervisor, for providing guidance on some of the more analytical aspects of the work I've done. I must also thank Agostino Patella for his many helpful conversations and computations. Maurizio Piai's lectures on Technicolor [1] and Thomas Pickup's DPhil thesis [2] influenced the introductory chapters to this thesis.

Thanks additionally to Claudio Pica, Antonio Rago, Luigi del Debbio, and all others involved, for the use of both the HiRep code and the two-flavour configurations, to Andreas Athinodorou and Georg Bergner for their contributions to the one-flavour work, and to Alan Irving at Liverpool for the use of the ULGQCD cluster.

Thanks also to Kirk Jordan and his team at IBM Watson Research Center in Cambridge for hosting me for a summer and for access to the Blue Gene/P and /Q machines in Yorktown.

This work was funded by STFC. Many of the computations for this work were performed on the 'pyxis' Blue Gene/P machine in Swansea University, part of the DiRAC facility, supported by STFC. Some computations were performed using the HPC Wales clusters, funded by the European Regional Development Fund through the Welsh European Funding Office, part of the Welsh Government.

Finally, thanks to Natalie for her support and encouragement. 


\section{List of Figures}

2.1 Sketches of the $\beta$ function in each of the regions discussed in the text. . 10

2.2 A cartoon showing the cases of theory one encounters as $N_{\mathrm{f}}$ is varied. $\quad 11$

2.3 Examples of rainbow and ladder diagrams, which are the basis of the rainbow/ladder approximation. These are equivalent numerically; the two shapes give rise to the common names given to the approximation. .

3.1 A three-dimensional example of a lattice setup. Lattice sites are in black; in blue are links which connect adjacent sites; the closed green paths are plaquettes. . . . . . . . . . . . . . . . . . . . 22

3.2 A sketch illustrating the truncation principle. The function of interest has a long tail before it "truly" reaches its asymptote, but rapidly reaches within $5 \%$ of it (in less than a fifth of the time); computations thus could be spared by computing to equilibrium enough to characterise a truncation length and correction, and subsequently truncating the measurements.

3.3 Smeared links are found by adding the original link (labeled as (1)) to the parallel transported nearest neighbours (labeled as (2)), weighted by a factor of $\alpha$, and the next-to-nearest-neighbours (labeled as (3)), weighted by a factor of $\beta \ldots \ldots \ldots \ldots$

3.4 Blocked links are found by summing spatial "staples" and adding the result to the direct (straight line) path; this procedure is then iterated. . 34

3.5 The histogram of the Polyakov loop of one of the data sets studied in chapter 4 ; the single peak seen shows that it is centre symmetric. . . . . 37

3.6 An effective mass plot for a meson mass; the plateau in this case was identified between $t=9$ and $t=16$. 


\section{LIST OF FIGURES}

3.7 The light meson spectrum of lattice QCD, as of 2008, from [3]. . . . . . 42

3.8 The ("gold-plated") heavy meson spectrum of lattice QCD, as of 2012, from $[4] \ldots \ldots \ldots \ldots$

3.9 The baryon spectrum from lattice QCD, as of 2009, from [5]. . . . . . 43

3.10 Comparison of various experimental methods with the lattice result for

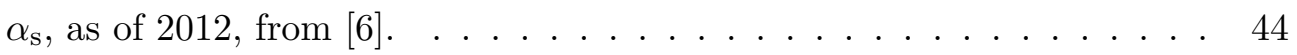

3.11 Exploration of the parameter space of candidate Technicolor theories, identifying their behaviour as conformal, confining, or indeterminate, from $[7] \ldots \ldots \ldots \ldots \ldots \ldots \ldots$

4.1 The phase diagram of the theory, showing the average plaquette across a range of value of $\beta$ and $m$; the region of interest was identified around

4.2 The run history for the D1 lattice; the plaquette is shifted vertically to improve the scaling on the $y$-axis. . . . . . . . . . . . . . . . . . . 64

4.3 Raw spectroscopic results for the pseudoscalar and vector meson masses, the $0^{++}$glueball, the spin- $\frac{1}{2}$ state, and the fundamental string tension. .

4.4 Spectroscopic results, showing the pseudoscalar, scalar, vector, and axial vector mesons, the spin- $\frac{1}{2}$ state, and the $0^{++}$glueball, normalised by the string tension, as a function of the PCAC fermion mass. At each bare fermion mass, only the data from the largest available lattice is shown (with the exception of the scalar state at $m=-1.51$, where the B3 results are used over the $\mathrm{C} 4$ results due to significantly lower errors). Approximate plateaux are also shown. . . . . . . . . . . . 66

4.5 Plateaux of various observables on the $32 \times 16^{3}$ lattice. . . . . . . . . . 67

4.6 Spectroscopic results, showing the pseudoscalar meson and $0^{++}$glueball, for the finite-volume study. . . . . . . . . . . . . . . . . . . 68

4.7 The static fermion potential, (a) for the four finite-size runs at $m=$ -1.51 (A4, B3, C4, D1), and the D2 run at $m=-1.523$, and (b) for the D2 run only, with the fitted curve included. . . . . . . . . . 68

4.8 (a) The quantity $\bar{\nu} / V$ for the B1, B2, B3, C5, C6, and D2 lattices; (b) The results for the mode number from the D2 lattice, compared with two possible fit results. . . . . . . . . . . . . . . . . . . . . . 
4.9 Plateaux for the fitted observables for the D2 data at various lengths and positions of the fitting window. The colour represents the position of the lower end of the window, and the $x$-axis the upper end. . . . . 71

5.1 Variation of the quantity $\chi^{\frac{1}{4}} / \sqrt{\sigma}$ with $\sigma$ for $\mathrm{SU}(n)$ theories, from [8]. . . 81

5.2 Instanton size distributions for $\mathrm{SU}(2)$ and $\mathrm{SU}(3)$, from [9] . . . . . . . . 81

5.3 Variation of the quantity $\chi^{\frac{1}{4}} / \sqrt{\sigma}$ with $\sigma \ldots \ldots \ldots \ldots$. . . . . . 82

5.4 Instanton size distribution for one- and two-flavour configurations on the $24 \times 12^{3}$, for one-flavour configurations on the $48 \times 24^{3}$ lattices, and for the pure gauge configurations. In all cases the distributions have been truncated at $\rho=\frac{2}{3} L_{T}$ to avoid boundary effects. . . . . . . . 83

5.5 Instanton size distribution for some of the one- and two-flavour runs on the $16 \times 8^{3}$ lattice, exhibiting severe finite-size effects, and the $20^{4}$ pure gauge data, exhibiting cooling artefacts at small instanton size. . . . . . 83

5.6 Comparison between the instanton size distributions of one- and twoflavour configurations affected and not affected by finite-size effects. . . 84

5.7 Variation of the average instanton size with $\sigma . \ldots \ldots \ldots$

5.8 Fitting the topological susceptibility for SU(2)Adj Nf1. The closest fit is found at $\gamma_{*} \approx 5.0$, which is well outside the acceptable bounds on $\gamma_{*}$, $0 \leq \gamma_{*}<2 \ldots \ldots \ldots \ldots \ldots \ldots \ldots \ldots$

5.9 Fitting the average instanton size for SU(2)Adj Nf1. Again, the closest fit is found at $\gamma_{*} \approx 5.0 \ldots \ldots \ldots \ldots$. . . . . . . . . . 88

5.10 Fitting the topological susceptibility for MWT. No good fit is apparent. 89

5.11 Fitting the average instanton size for MWT. All plots fit, since the error bars are larger than the deviation in the data; no bounds may be placed on $\gamma_{*} \ldots \ldots \ldots \ldots \ldots \ldots \ldots \ldots \ldots \ldots$

6.1 Results of the spinor field square norm test plotted for the whole ensemble.105

6.2 Results of the spinor field square norm test, plotting the average performance per MPI process. . . . . . . . . . . . . . . . . 106

6.3 Results of the spinor field multiply-add, plotted for the whole ensemble. Symbols as on page 105 . . . . . . . . . . . . . . . . . 107

6.4 Results of the spinor field multiply-add, plotting the average performance per MPI process. Symbols as on page 105 . . . . . . . . . . . . . 108 


\section{LIST OF FIGURES}

6.5 Results of the Dirac operator application test, plotted for the whole ensemble. Symbols as on page 105. . . . . . . . . . . . . . . . 109

6.6 Results of the Dirac operator application test, plotting the average performance per MPI process. Symbols as on page 105. . . . . . . . . . . 110

6.7 Total combined results of all three tests, plotted for the whole ensemble. Symbols as on page 105. . . . . . . . . . . . . . . . . . . 111

6.8 Total combined results of all three tests, plotting the average performance per MPI process. Symbols as on page 105 . . . . . . . . . . . . 112

6.9 Rack-for-rack comparison between Blue Gene/P and Blue Gene/Q performance, using a larger lattice size in the case of the Blue Gene/Q to allow use of a full rack. Symbols as on page 105. . . . . . . . . . . . 113

6.10 Performance comparison of a single Blue Gene/Q partition at various subdivisions; from one process per CPU through to 64 processes per CPU (four per core). Symbols as on page 105 . . . . . . . . . . . . . . 113

A.1 The topological charge distribution of a single timeslice, before and after cooling. Rendering was performed using a Mac Pro machine in Swansea University as the frontend, and the BlueIce2 cluster in Swansea University as the backend, using 480 compute cores across 40 nodes. . . . . . 122

A.2 The topological charge distribution of a single timeslice. Moving anticlockwise around the ring takes us from the uncooled case to 10 cools. Rendering was performed using the Mac Pro used in figure A.1 as both front and backend. . . . . . . . . . . . . . . . . . . . . . . . 122

A.3 The topological charge distribution of all 64 timeslices of the configuration. Moving around the ring takes us around the time dimension, while moving down the columns takes us from the uncooled state to 20 cools. This gives a total of 1344 cubes. Rendering was performed using the machine combination used for figure A.1. . . . . . . . . . . . . . 123 


\section{List of Tables}

4.1 The terms in equation (4.24) that do not cancel are marked with a $\checkmark . \quad 54$

4.2 A summary of the important results of section 4.1 . . . . . . . . . 60

4.3 Lattices considered and parameters used. In the Machine column, SC refers to an x86-64 cluster in Swansea University, UL to the ULGQCD machine in Liverpool, and BGP to the 'pyxis' Blue Gene/P machine (part of the STFC DiRAC facility) in Swansea University. . . . . . . . . 62

4.4 PCAC and pseudoscalar meson mass. . . . . . . . . . . . . 62

4.5 Glueball masses and string tension, as computed by Biagio Lucini and Andreas Athenodorou. . . . . . . . . . . . . . . . . 63

4.6 Masses of the spin- $\frac{1}{2}$ particle and of the scalar, vector, and axial vector mesons (including the disconnected contribution where appropriate), as computed by Georg Bergner. . . . . . . . . . . . . . . . . . . . 63

4.7 Results of the fit of the static potential from Wilson loops as $V(r)=$ $a+b / r+\sigma r$, with $\sigma$ set to the value found from Polyakov loop correlators. 63

5.1 Numerical results for $m_{P C A C}, \chi$ and $\bar{\rho}$ for $N_{\mathrm{f}}=1$ as a function of $m_{\mathrm{PCAC}}$ on lattices of size $2 L \times L^{3}$. Lattice names correspond with those in chapter $4 \ldots \ldots \ldots \ldots \ldots$. . . . . . . . . . . . 86

5.2 Numerical results for $m_{P C A C}, \chi$ and $\bar{\rho}$ for MWT as a function of $m_{\mathrm{PCAC}}$ on lattices of size $2 L \times L^{3}$. Lattice names correspond with those in [10]. 86

6.1 The seven machines tested by BSMBench; the lines divide the machines into the groups in which they were plotted. . . . . . . . . . . . . 103 


\section{Chapter 1}

\section{Introduction}

It is an exciting time to be working in particle physics. With the results from the LHC flowing in, and revealing that nature has a scalar state with a mass of $125 \mathrm{GeV}$, there is a focus within the theoretical community to explain these results, beyond the predictions of Higgs $[11,12]$. Many tools are used for this, including analytic (but often approximate) methods such as gauge-gravity duality, and first-principles computational methods like the lattice approach. The work presented in this thesis looks at a family of theories known as Technicolor, which makes use of strong dynamics to break the electroweak symmetry, using the lattice approach. Related to this, it also looks at ways of measuring the performance of supercomputers at performing lattice computations for these theories; this is an important step when procuring machines to do work such as that presented in this thesis.

In chapter 2, I will motivate and describe Technicolor as a family of theories. Then in chapter 3, I will introduce the framework of lattice gauge theory, and the algorithms therein that have been used, review recent results, and describe what is known of the two theories to be studied in the following chapters. Chapter 4 describes the investigation of a particular theory of relevance for Technicolor models, namely the SU(2) gauge theory with one adjoint Dirac flavour (which I will refer to as SU(2)Adj Nf1), the first lattice investigation of this theory. The study is exploratory in nature, looking at the spectroscopy of the theory as well as the static potential and string tension from Wilson and Polyakov loops, and the anomalous dimension of the chiral condensate from the Dirac mode number. The results tentatively point at the theory not being traditionally 


\section{INTRODUCTION}

confining; instead lying within or near the conformal window. Some of the work in this chapter was previously presented in a poster at the SEWM conference [13].

Chapter 5, meanwhile, after reviewing some aspects of instantons and topology, looks at the topology of this theory, and that of another $\mathrm{SU}(2)$ gauge theory: that with two adjoint Dirac flavours, known as Minimal Walking Technicolor. Previous studies have looked at the spectroscopy and running of the coupling of this theory; the topology observed is found to be consistent with that expected from previous observations (based on other observables) - in both theories it is consistent with conformal or near-conformal behaviour - and it is also found that the instanton size distribution may be useful as an indicator of the onset of finite-volume effects. Scaling behaviour found for other observables in previous work is however not observed in the topological observables considered here. Some of the work in this chapter was previously published in [14].

Next, chapter 6, after reviewing some relevant aspects of high-performance computers, briefly describes the HiRep code, which has been used extensively throughout this work, and then details BSMBench, a supercomputing benchmark tool based on HiRep to support the investigation of new physics by allowing an informed choice of equipment purchase, as well as having applications beyond physics. Some of the work in this chapter was previously presented in a poster at ISC'12 [15]. Finally, chapter 7 sums up the findings of the work presented in the preceding chapters, and gives an outlook for potential future work in this field.

Two appendices are also included: the first includes visualisations of topological charge distributions based on the work of chapters 4 and 5, which were submitted for Swansea University's Research as Art and the Royal Society's Picturing Science competitions. The second appendix includes the README and LICENSE text files for the BSMBench software. 


\section{Chapter 2}

\section{The Standard Model and Novel Strong Interactions}

The Standard Model accurately describes almost all known interactions of elementary particles (with the notable exception of gravity, treated separately) to an exquisitely high precision - for example, the anomalous magnetic moment of the electron, $(g-2) / 2$, has been calculated analytically to 3-loop order [16], and numerically to ten-loop order to have a value of $1.15965218178(77) \times 10^{-3}$ [17], which is in exact agreement with the experimental value of $1.15965218076(27) \times 10^{-3}$ [18] (itself one of the most precise experimental measurements ever made). Its most recent success was the prediction of the Higgs particle; in July 2012 the ATLAS and CMS teams at the Large Hadron Collider at CERN announced the discovery of a particle matching the description of the Higgs - that is, a scalar boson, found to have a mass of $125 \mathrm{GeV}[19,20]$.

\subsection{The Standard Model}

The (minimal) standard model (MSM) is formulated in the framework of Quantum Field Theory. It has a $\mathrm{SU}(3) \times \mathrm{SU}(2) \times \mathrm{U}(1)$ gauge symmetry, and the following field content: 


\begin{tabular}{|c|c|c|c|c|c|}
\hline Field & Description & $\begin{array}{l}\text { Gauge } \\
\text { boson }\end{array}$ & $\begin{array}{c}\mathrm{SU}(3) \\
\text { representation }\end{array}$ & $\begin{array}{c}\mathrm{SU}(2) \\
\text { representation }\end{array}$ & $\begin{array}{c}\mathrm{U}(1) \\
\text { Hypercharge }\end{array}$ \\
\hline$B$ & Weak hypercharge & $\mathrm{B}^{0}$ & 1 & 1 & 0 \\
\hline$W$ & Weak isospin & $\mathrm{W}^{ \pm}, \mathrm{W}^{0}$ & 1 & 3 & 0 \\
\hline$G$ & Colour & Gluons & 8 & 1 & 0 \\
\hline$Q_{\mathrm{L}}$ & Left-handed quark & & 3 & 2 & $\frac{1}{6}$ \\
\hline$u_{\mathrm{R}}$ & Left-handed up quark & & 3 & 1 & $\frac{2}{3}$ \\
\hline$d_{\mathrm{R}}$ & Left-handed down quark & & 3 & 1 & $-\frac{1}{3}$ \\
\hline$L_{\mathrm{L}}$ & Left-handed lepton & & 1 & 2 & $-\frac{1}{2}$ \\
\hline$E_{\mathrm{R}}$ & Right-handed electron & & 1 & 1 & -1 \\
\hline$H$ & Elementary Higgs $^{1}$ & & 1 & 2 & $\frac{1}{2}$ \\
\hline
\end{tabular}

Its Lagrangian takes the general form ${ }^{2}$

$$
\mathscr{L}_{\mathrm{SM}}=-\frac{1}{2} \operatorname{tr} F^{2}+\bar{\psi} i \not D \psi+(D H)^{\dagger} D H-Y \bar{\psi} H \psi-V\left(H^{\dagger} H\right),
$$

with the terms representing, in turn:

- the kinetic contribution of the spin-1 gauge fields,

- the kinetic contribution of the fermion fields, and interactions described by the $\mathrm{SU}(3) \times \mathrm{SU}(2) \times \mathrm{U}(1)$ group,

- the kinetic contribution of the Higgs and its interactions with the gauge fields,

- the Yukawa interactions between the Higgs and the fermions, and

- the self-interaction of the Higgs.

\subsubsection{Breaking Electroweak Symmetry with a Fundamental Scalar}

Noticeable in the field composition of the MSM is the lack of a photon; this is due to the fact that the electroweak symmetry $\mathrm{SU}(2) \times \mathrm{U}(1)$ is broken at low energies to the U(1) symmetry of QED. In the MSM, this breaking takes place via the elementary

\footnotetext{
${ }^{1}$ It is the introduction of the elementary Higgs (also known as the Standard Model Higgs) here which takes us from the Standard Model to the Minimal Standard Model

${ }^{2}$ This is a schematic rather than quantitative representation, since for instance the left- and righthanded fermions are treated separately, and the quark states seen by the strong interaction are different from those acted on by the weak interaction. However, reducing to the simplest form in this way will aid the discussion, since there will be fewer distractions from unnecessary details.
} 
Higgs, through a mechanism proposed by Weinberg and Salam [21, 22], who made use of prior work by Glashow [23], and the Higgs mechanism, developed by various authors $[11,12,24,25]$. Its potential takes the form of a complex scalar $\phi^{4}$ interaction:

$$
V(H)=-\mu^{2} H^{\dagger} H+\frac{1}{4} \lambda\left(H^{\dagger} H\right)^{2}
$$

This potential has the familiar "Mexican hat" shape, and thus fails to have a minimum at $H=0$; thus $H$ obtains a non-zero vacuum expectation value (VEV). Since $H$ transforms under the $\mathrm{SU}(2)$ symmetry, this symmetry is spontaneously broken: the breaking pattern is $\mathrm{SU}(2)_{L} \times \mathrm{U}(1)_{Y} \rightarrow \mathrm{U}(1)_{Q}$ (where subscripts have been added to clarify that both hypercharge and weak isospin are broken, leaving electric charge).

Goldstone's theorem [26] tells us that this breaking will reward us with three Goldstone bosons, which via the Higgs mechanism are consumed by the $\mathrm{W}^{ \pm}$and $\mathrm{Z}$ bosons to become their longitudinal degrees of freedom; this then allows the gauge bosons to become massive. Looking also at the Yukawa interactions, we can see that once the broken symmetry has given $H$ a non-zero $\mathrm{VEV}$, then these interactions give a mass term to the Standard Model fermion fields. A detailed treatment of why this is so is deferred to a textbook on QFT (for example [27]).

Looking back at $H$, we notice that of the four degrees of freedom it had, only three have been absorbed by $\mathrm{W}^{ \pm}$and $\mathrm{Z}$; this leaves one fundamental massive scalar waiting to be detected. It is this prediction of an additional boson that led to the search for one at the LHC; the discovery of a particle consistent with these properties is a strong indication that the theory is either correct or very close to the mark. Additionally, the work of the Particle Data group [18] collates over 27,000 independent measurements from experiments across the planet, all of which are in agreement with the predictions of the Standard Model, many with extremely high precision.

\subsubsection{Problems with the Standard Model}

If we have a theory as good as is suggested by the end of the last section, then we face an obvious question: why would we be in need of a replacement? The most obvious omission from the Standard Model is the lack of any treatment of gravity. Although this is an issue for a fundamental theory of nature, in practice, this has not proved too large a problem for understanding experimental results. All accessible physics can 


\section{THE STANDARD MODEL AND NOVEL STRONG INTERACTIONS}

be explained by considering the SM with no gravity, and then using classical gravity or general relativity (and macroscopic SM results) at larger scales. The problems remaining are exotic situations which we cannot (currently) probe experimentally, and a rather more fundamental problem known as the hierarchy problem.

Working on the assumption that there is a consistent theory explaining all fundamental forces (even though we don't know it), we expect it to "switch on" at some scale. We know that this must be at or below the Planck scale $\left(\sim 10^{19} \mathrm{GeV}\right)$, since this is where quantum field theory in general breaks down. If we assume that no new physics occurs between EWSB and the Planck scale, then we find ourselves with a problem: the mass of the Higgs required to make the Standard Model consistent with observed results (not to mention the one observed) is many orders of magnitude smaller than the only scale we have to work with, the Planck scale. Such a problem is referred to as a hierarchy problem. Historically, when inconsistencies in a working theory have been found, they have been solved by conjecturing (and, in general, subsequently discovering) new physics (with associated new scales), and it seems reasonable that in the many orders of magnitude that are unexplored, some new physics lies waiting to be found.

The other remaining issue is that of the five terms in eq. (2.1), the 27,000 measurements mentioned above all relate to the first two. The remaining three - all relating to the Higgs sector, which is not represented in the two terms that have been probedare essentially untested. With the discovery of the Higgs at the LHC, experiment will hopefully be able to begin putting bounds on them. To this end, it is prudent to develop a variety of alternative theories (that leave the observed results unchanged, or at least within error bars), and experimental signatures for their correctness, so that they may be distinguished.

For these reasons it is important to look beyond the theory of the Standard Model to explore the space of possible theories, to find models that will solve these problems. There are a number of popular candidates, which have developed over the last few decades and inspired whole fields of research. They include supersymmetry the introduction of a new boson-fermion symmetry, creating "superpartners" for all SM particles), a theory which has proven a useful tool in building toy models outside of its potential for being found as new physics, and extra-dimensional models such as Randall-Sundrum (where the universe as we observe it is localised on a 3+1-dimensional 
slice of a higher-dimensional space, whose dynamics account for unexplained phenomena). The work presented in this thesis looks at two theories falling in a class that have the potential to solve many of the Standard Model's shortcomings-known in general as Technicolor ${ }^{1}$, which is described in the remainder of this chapter - and also looks at technology being developed for the investigation of new theories.

\subsection{Technicolor}

Technicolor (sometimes abbreviated to TC) is an alternative theory of EWSB proposed independently by Weinberg $[28,29]$ and Susskind [30] in 1979. The central idea is that rather than breaking via a fundamental scalar Higgs, the electroweak symmetry is broken dynamically by the generation of a VEV for a chiral condensate $\langle\bar{\psi} \psi\rangle$ through chiral symmetry breaking.

This behaviour can be demonstrated even in QCD, as was noted in [31]. The QCD Lagrangian takes the form

$$
\mathscr{L}_{\mathrm{QCD}}=-\frac{1}{4} F_{\mu \nu}^{a} F_{a}^{\mu \nu}+\bar{\psi}_{i}\left(\not_{i j}^{m=0}-m \delta_{i j}\right) \psi_{j}
$$

where $F_{\mu \nu}^{a}$ are the field strengths of the gluon fields, which lie in the adjoint representation of QCD's SU(3) gauge group, $\psi_{i}$ are quark fields, Dirac fermions in the fundamental representation of the gauge group, $\not D^{m=0}$ is the massless Dirac operator, characterising both the kinetic behaviour of the quarks and their interactions with the gluons, and $m$ is the quark mass (which is set to be equal for all flavours of quarks considered).

If we decompose the quark fields into left- and right-handed chirality components using the projection operators $P_{\mathrm{R}(\mathrm{L})}=\frac{1}{2}\left(1 \pm \gamma_{5}\right)$, defining $\psi_{\mathrm{R}(\mathrm{L})}=P_{\mathrm{R}(\mathrm{L})} \psi$, where $\psi=\psi_{\mathrm{L}}+\psi_{\mathrm{R}}$, then the Lagrangian becomes

$$
\mathscr{L}_{\mathrm{QCD}}=-\frac{1}{4} F_{\mu \nu}^{a} F_{a}^{\mu \nu}+\bar{\psi}_{\mathrm{L} i} \not D_{i j}^{m=0} \psi_{\mathrm{L} j}+\bar{\psi}_{\mathrm{R} i} \not D_{i j}^{m=0} \psi_{\mathrm{R} j}-m\left(\bar{\psi}_{\mathrm{L} i} \psi_{\mathrm{R} i}+\bar{\psi}_{\mathrm{R} i} \psi_{\mathrm{L} i}\right)
$$

Note that the only term that mixes left- and right-handed states is the mass; in the limit of massless quarks then the states are independent, and the Lagrangian is invariant

\footnotetext{
${ }^{1}$ Strictly speaking, the name "Technicolor" applies only to the first theory described in the next section, the related family of theories being properly called "strongly interacting BSM dynamics" or similar; however, the name Technicolor has also stuck as a slightly unfortunate name for the family, due to its shorter length than the alternative, and it will be used as such throughout this work.
} 


\section{THE STANDARD MODEL AND NOVEL STRONG INTERACTIONS}

under $\mathrm{U}\left(N_{\mathrm{f}}\right)$ flavour transformations applying independently to left- and right-handed components:

$$
\psi_{\mathrm{L}(\mathrm{R}) i} \mapsto \psi_{\mathrm{L}(\mathrm{R}) i}^{\prime}=U_{i j}^{\mathrm{L}(\mathrm{R})} \psi_{\mathrm{L}(\mathrm{R}) j}, \quad U^{\mathrm{L}(\mathrm{R})} \in \mathrm{U}\left(N_{\mathrm{f}}\right)
$$

which gives an overall symmetry group $\mathrm{U}\left(N_{\mathrm{f}}\right) \times \mathrm{U}\left(N_{\mathrm{f}}\right)$. We may split out $\mathrm{U}(1)$ components from the groups, and then take linear combinations $\mathrm{V}=\mathrm{R}+\mathrm{L}, \mathrm{A}=\mathrm{R}-\mathrm{L}$, to give a total global symmetry group of $\mathrm{SU}(2)_{\mathrm{L}} \times \mathrm{SU}(2)_{\mathrm{R}} \times \mathrm{U}(1)_{\mathrm{V}} \times \mathrm{U}(1)_{\mathrm{A}}$, where we have now restricted ourselves to considering two quark flavours.

At a quantum level, the $\mathrm{U}(1)_{\mathrm{A}}$ is subject to the well-known axial anomaly (see, for example, $[32,33]$, or any recent QFT textbook, for instance [34]), reducing the symmetry to $\mathrm{SU}(2)_{\mathrm{L}} \times \mathrm{SU}(2)_{\mathrm{R}} \times \mathrm{U}(1)_{\mathrm{V}}$. But if we then move to strong coupling, then the vacuum will spontaneously produce quark-antiquark pairs, giving a VEV to the fermionic condensate - which by comparison with equation (2.4) we see breaks the chiral symmetry: only the vectorial subgroup remains, as $\mathrm{SU}(2)_{\mathrm{L}} \times \mathrm{SU}(2)_{\mathrm{R}} \rightarrow \mathrm{SU}(2)_{\mathrm{V}}$.

To connect this with EWSB, we gauge the $\mathrm{SU}(2)_{\mathrm{L}} \times \mathrm{U}(1)_{Y}$ subgroup of $\mathrm{SU}(2)_{\mathrm{L}} \times$ $\mathrm{SU}(2)_{\mathrm{R}}{ }^{1}$; now the chiral symmetry breaking pattern is $\mathrm{SU}(2)_{\mathrm{L}} \times \mathrm{U}(1)_{Y} \rightarrow \mathrm{U}(1)_{Q}$. Since we have broken a gauge rather than a global symmetry, the Goldstone bosons are absorbed into the gauge bosons as in the fundamental Higgs case.

In fact, QCD does couple to the electroweak sector in this manner, and its chiral symmetry breaking gives a contribution to the masses of $\mathrm{W}^{ \pm}$and $\mathrm{Z}$; unfortunately this contribution is decidedly small, so we must look to other theories to break EWSB and generate the remainder of the mass.

Conspicuous by its absence here is the residual scalar state seen in the case of EWSB by an elementary Higgs; given the recent discovery of such a state at the LHC we are prompted to question the value of Technicolor as a theory, since it appears incompatible with this discovery. We will see in the coming sections how this particular bullet is dodged.

\subsubsection{Beyond QCD: Phases of SU $(N)$ Gauge Theories}

Looking back at the QCD Lagrangian (equation (2.3)), we can see a number of parameters that seem have been "set" to get this theory, which could be altered to give a theory

\footnotetext{
${ }^{1} \mathrm{The} \mathrm{U}(1)_{Y}$ is generated by the third generator of $\mathrm{SU}(2)_{\mathrm{R}}$, to connect to the electroweak chiral Lagrangian $[1,35,36]$.
} 
lying "beyond" QCD; the most apparent are the gauge group (which could be altered to another $\mathrm{SU}(N)$ group, or some other group entirely), the number of fermions, and the fermion representation (why not a "higher" representation?). It is this parameter space that is being explored to look for potential Technicolor theories; to this end, it is important to understand the properties of gauge theories in various areas of this space.

This phase space was explored perturbatively, at least for $\mathrm{SU}(N)$ theories, by Dietrich and Sannino in [37], of whose results I will summarise some here. The explanation relies upon the Renormalisation Group (RG) beta-function $\beta(g)$, and the anomalous dimension of the chiral condensate $\gamma_{*}$, which are defined as:

$$
\begin{aligned}
\beta(g) & =\frac{\partial g}{\partial \ln (\mu)} \equiv \mu \frac{\partial g}{\partial \mu} \\
\gamma_{*} & =\frac{\partial \ln \langle\bar{\psi} \psi\rangle_{\mu}}{\partial \ln \mu} \equiv \mu \frac{\partial \ln \langle\bar{\psi} \psi\rangle_{\mu}}{\partial \mu}
\end{aligned}
$$

where $\mu$ is the RG scale. At $\beta(g)=0$, the theory has an RG fixed point.

The two-loop beta-function of a general non-Abelian gauge theory, with fermionic matter in some representation of $\mathrm{SU}(N)$ labeled $\mathrm{R}$ takes the form [38]:

$$
\beta(g)=-\beta_{0} \frac{g^{3}}{(4 \pi)^{2}}-\beta_{1} \frac{g^{5}}{(4 \pi)^{4}},
$$

where

$$
\begin{aligned}
2 N \beta_{0} & =\frac{11}{3} C_{2}(\mathrm{G})-\frac{4}{3} T(\mathrm{R}) \\
(2 N)^{2} \beta_{1} & =\frac{34}{3}\left(C_{2}(\mathrm{G})\right)^{2}-\frac{20}{3} C_{2}(\mathrm{G}) T(\mathrm{R})-4 C_{2}(\mathrm{R}) T(\mathrm{R}),
\end{aligned}
$$

where in turn, $C_{2}(\mathrm{R})$ is the quadratic Casimir operator of the representation $\mathrm{R}$ (with $\mathrm{G}$ referring to the adjoint), and $T(\mathrm{R})$ is the trace normalisation factor for that representation; for $N_{\mathrm{f}}$ flavours,

$$
\begin{aligned}
2 N X_{\mathrm{R}}^{a} X_{\mathrm{R}}^{a} & =C_{2}(\mathrm{R}) \\
N_{\mathrm{f}} C_{2}(\mathrm{R}) \operatorname{dim}(\mathrm{R}) & =T(\mathrm{R}) \operatorname{dim}(\mathrm{G}) .
\end{aligned}
$$

$X_{\mathrm{R}}^{a}$ refers to the the generators of the group in the representation R; equation (2.12) is derived in Jones [39], and $\operatorname{dim}(\mathrm{R})$ refers to the dimension of the representation. 


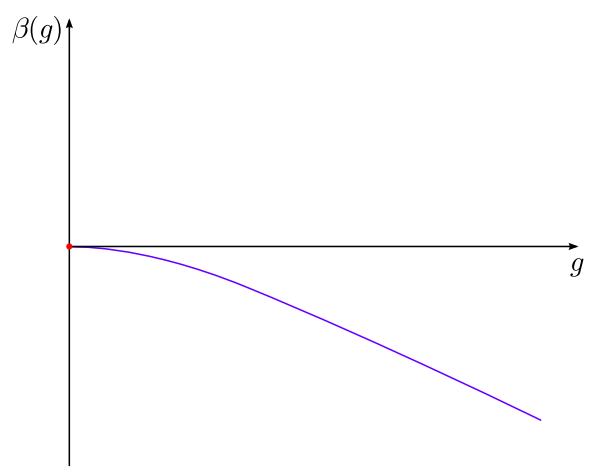

(a) Confining

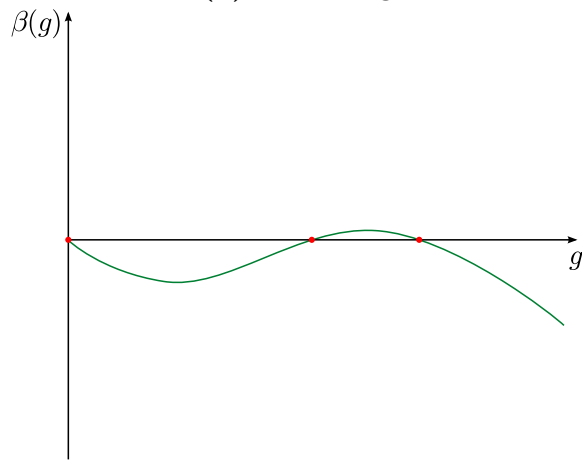

(c) Conformal

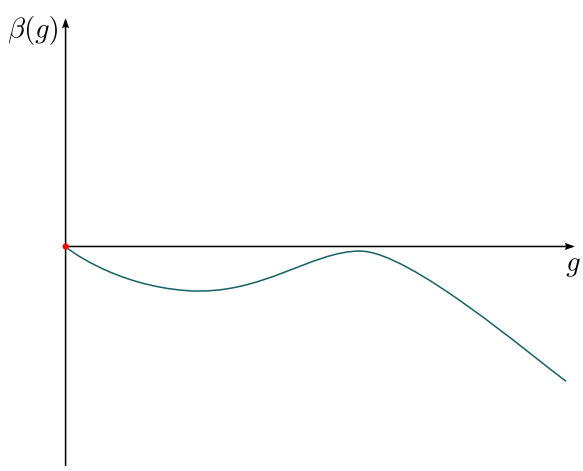

(b) Near-conformal

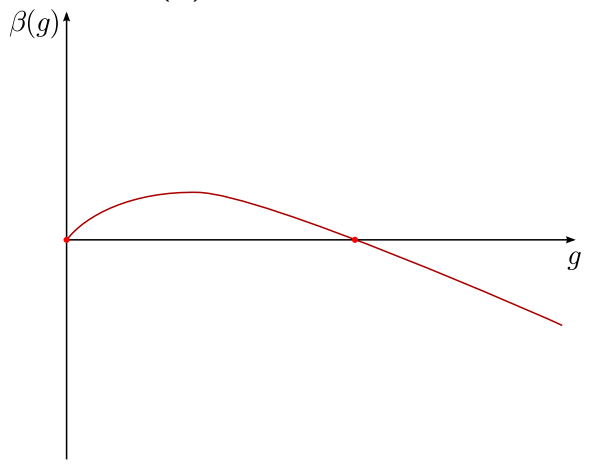

(d) Asymptotic freedom lost

Figure 2.1: Sketches of the $\beta$ function in each of the regions discussed in the text.

Thus as the number of flavours is raised, the shape of the $\beta$ function changes. Above some value of $N_{\mathrm{f}}$, which Dietrich and Sannino refer to as $N_{\mathrm{f}}^{\mathrm{I}}[\mathrm{R}]$, the sign of $\beta_{0}$ changes from negative to positive, giving a theory which no longer has asymptotic freedom. By rearranging $(2.9)$, we see

$$
N_{\mathrm{f}}^{\mathrm{I}}[\mathrm{R}]=\frac{11}{4} \frac{\operatorname{dim}(\mathrm{G}) C_{2}(\mathrm{G})}{\operatorname{dim}(\mathrm{R}) C_{2}(\mathrm{R})}
$$

which can be immediately seen to have a fixed value of $11 / 4$ for the adjoint representation. Meanwhile moving from the other direction, we can see that at another value of $N_{\mathrm{f}}$, which Dietrich and Sannino call $N_{\mathrm{f}}^{\mathrm{III}}[\mathrm{R}]$, the $\beta_{0}$ and $\beta_{1}$ terms will cancel, resulting in a conformal fixed point first discussed by Caswell [38], and later by Banks and Zaks [40].

However, whether this conformal fixed point can be reached depends on another factor, pointed out by $[41,42]$ and discussed in the next section: the relative order of 
$\alpha_{*}$ - the value of the coupling constant where the fixed point is encountered - and $\alpha_{\mathrm{c}}-$ where chiral symmetry breaking occurs. Looking at the RG flow to the IR, if the fixed point at $\alpha_{*}$ is reached first, then the coupling freezes, so $\alpha_{\mathrm{c}}$ will never be reached, and chiral symmetry will not break. Meanwhile, if $\alpha_{\mathrm{c}}$ is reached first, then chiral symmetry breaks and the fermions decouple; the lack of screening by fermions to counteract the antiscreening of gauge bosons prevents the fixed point from being reached. The point at which the relative order changes is referred to by Dietrich and Sannino as $N_{\mathrm{f}}^{\mathrm{II}}[\mathrm{R}]$.

To summarise, working from high to low $N_{\mathrm{f}}$, we have a region without asymptotic freedom, then an IR-conformal region with a BZ fixed point, then a confining region, which initially has a BZ fixed point that is almost reached; the closer $N_{\mathrm{f}}$ is to $N_{\mathrm{f}}^{\mathrm{II}}[\mathrm{R}]$, the closer the theory gets to the fixed point. This is illustrated in figure 2.2. The nearconformal region is, as it might appear, the region that is close to the conformal window but not inside it; this will come into play in the discussion of Walking Technicolor in a later next section.

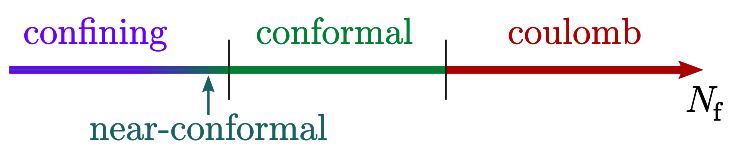

Figure 2.2: A cartoon showing the cases of theory one encounters as $N_{\mathrm{f}}$ is varied.

\subsubsection{Ladders and Rainbows}

In the previous section I discussed the need to ascertain where $\alpha_{*}=\alpha_{\mathrm{c}}$ in order to find $N_{\mathrm{f}}^{\mathrm{II}}[\mathrm{R}]$. At two-loop order in perturbation theory, $\alpha_{*}$ is trivial to find from equation 2.8 above:

$$
\alpha_{*}=\left.\frac{g^{2}}{4 \pi}\right|_{\beta(g)=0}=-\frac{\beta_{0}}{\beta_{1}} .
$$

$\alpha_{\mathrm{c}}$, meanwhile, requires a little more work. We can find it by making use of the Schwinger-Dyson (SD) equation for the fermion propagator, which for massless QCD has the form [42]

$$
S^{-1}(p)=i \not p+\int^{\Lambda} \frac{d^{4} q}{(2 \pi)^{4}} g^{2} D_{\mu \nu}(p-q) \frac{\lambda^{a}}{2} \gamma_{\mu} S(q) \Gamma_{\nu}^{a}(q ; p),
$$

where $\Lambda$ is the regularisation scale, $D_{\mu \nu}(k)$ the dressed renormalised gluon propagator, and $\Gamma_{\nu}^{a}(q ; p)$ is the renormalised, dressed vertex term. As is always the case in 


\section{THE STANDARD MODEL AND NOVEL STRONG INTERACTIONS}

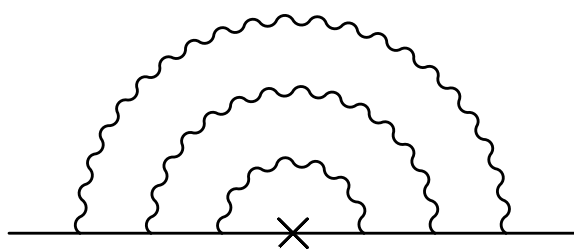

(a) Rainbow

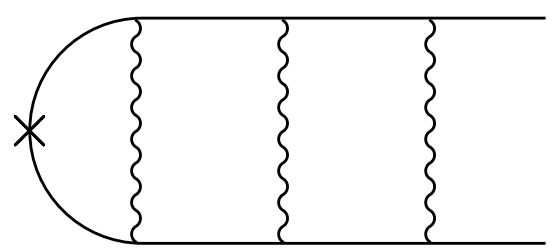

(b) Ladder

Figure 2.3: Examples of rainbow and ladder diagrams, which are the basis of the rainbow/ladder approximation. These are equivalent numerically; the two shapes give rise to the common names given to the approximation.

perturbation theory, the propagator is dressed by loop corrections, which are themselves dressed, in a recursive manner. The $i \not p$ contribution is from the bare fermion propagator, and the second term adds the dressing; here we see the recursion in $S$.

When dressed, the fermion propagator may be written as:

$$
S(p)=\frac{i}{Z(p) \not p-\Sigma(p)},
$$

where $\Sigma(p)$ is the self-energy of the fermion, and $Z(p)$ the wave function renormalisation; however, inserting this into (2.15) does not solve the recursion problem. The dressing must be approximated to make the analysis tractable, whilst retaining the interesting physics. This can be done by making use of by the "rainbow" or "ladder" approximation; these refer to the same approximation, drawn in two different ways, as shown in figure 2.3, which was first used in the context of QED in [43]. This approximation replaces the dressed gluon propagator and vertex term by their tree-level values, which when combined with (2.16) allows coupled equations for $Z(p)$ and $\Sigma(p)$ to be written. From this, [42] shows that $Z(p)=1$ for all $\Sigma$; this then allows us to write

$$
\Sigma(p)=3 g^{2} C_{2}(\mathrm{R}) \int \frac{d^{4} k}{(2 \pi)^{4}} \frac{1}{(p-k)^{2}} \frac{\Sigma(k)}{k^{2}+\Sigma(k)^{2}} .
$$

This is obviously still recursive, but only in $\Sigma$, for which we may solve analytically. The critical case is taken to be that where the anomalous dimension of the condensate $\gamma_{*}=1 ;$ this is the case for

$$
\alpha_{\mathrm{c}}=\frac{\pi}{3 C_{2}(\mathrm{R})}
$$


Now by equating equations (2.14) and (2.18) we arrive at an estimate for the lower end of the conformal window, $N_{\mathrm{f}}^{\mathrm{II}}[\mathrm{R}]$ :

$$
N_{\mathrm{f}}^{\mathrm{II}}[\mathrm{R}]=\frac{\operatorname{dim}(\mathrm{G}) C_{2}(\mathrm{G})}{\operatorname{dim}(\mathrm{R}) C_{2}(\mathrm{R})} \frac{17 C_{2}(\mathrm{G})+66 C_{2}(\mathrm{R})}{10 C_{2}(\mathrm{G})+30 C_{2}(\mathrm{R})} .
$$

Note that this estimate has been obtained by combining two different approximations: perturbation theory, and a truncation of the Schwinger-Dyson equation; therefore, it should only be taken as an qualitative indication. A quantitative, precise prediction would require a first-principles non-perturbative approach: this is one of the subjects of current research in the field, as will be outlined below.

\subsubsection{Extended and Walking Technicolor}

We noticed previously that the most basic Technicolor theories fail to give a scalar state corresponding with the Higgs boson; however, there is also another problem that needs addressing: unlike the elementary Higgs, the techniquarks do not have any couplings to the SM fermions, and so EWSB does not generate any masses for them. This problem can be solved by introducing a new gauge group, with both SM fermions and techniquarks charged under it; this is referred to as Extended Technicolor (ETC), proposed in $[44,45]$. Looking at the resulting four-fermion vertices,

$$
\alpha_{a b} \frac{\bar{\Psi} T^{a} \Psi \bar{\Psi} T^{b} \Psi}{\Lambda_{\mathrm{ETC}}^{2}}+\beta_{a b} \frac{\bar{\Psi} T^{a} \Psi \bar{\psi} T^{b} \psi}{\Lambda_{\mathrm{ETC}}^{2}}+\gamma_{a b} \frac{\bar{\psi} T^{a} \psi \bar{\psi} T^{b} \psi}{\Lambda_{\mathrm{ETC}}^{2}}+\ldots
$$

where for the purposes of clarity in this section, $\Psi$ represents the technifermions, and $\psi$ the Standard Model fermions; $T^{a}$ are the generators of the flavour symmetries, and $a, b$ indices refer to the flavour sector, determining the precise nature of the ETC theory in question. Looking at the second term, it evidently mixes SM and technifermions; at $\Lambda_{\mathrm{TC}}$ the technifermions will form a condensate, giving an interaction of the form:

$$
\mathscr{L}_{\text {int }}=\beta_{a} \frac{\langle\bar{\Psi} \Psi\rangle_{\mathrm{ETC}} \bar{\psi}_{a} \psi_{a}}{\Lambda_{\mathrm{ETC}}^{2}},
$$

which provides the fermions with a mass. Now, since the $\beta$ coefficient results from two underlying vertices in the ETC theory, and that $\langle\bar{\Psi} \Psi\rangle \sim \Lambda_{\mathrm{TC}}^{3}$, the resulting SM 


\section{THE STANDARD MODEL AND NOVEL STRONG INTERACTIONS}

fermion masses will be of the order

$$
m_{\mathrm{f}} \sim \frac{g_{\mathrm{ETC}}^{2} \Lambda_{\mathrm{TC}}^{3}}{\Lambda_{\mathrm{ETC}}^{2}}
$$

Additionally, if we consider the possibility that rather than one ETC breaking scale, there are instead three, then we can see a natural way in which the three generations of the Standard Model fermions might arise - the three generations of quarks and leptons each have successively higher masses, which are now each associated with a separate breaking scale. This mechanism is referred to as tumbling [46].

The $\alpha$ terms, meanwhile, give masses to Goldstone bosons arising from the breaking that aren't consumed by the gauge bosons; in the absence of this term, their masses would be sufficiently small that we would expect to have observed them [45, 47] which we have not [18] - and the theory would be ruled out. However, looking at the $\gamma$ term we find we have introduced almost as many problems as we've solved, since we've added a new set of couplings between the SM fermions, which allows for flavourchanging neutral currents (FCNCs). FCNCs are highly constrained in observations; a sector that provides definitive limits is that of the neutral kaons, where the $\mathrm{K}^{0}$ and $\overline{\mathrm{K}^{0}}$ states mix, giving mass eigenstates $\mathrm{K}_{\mathrm{S}}^{0}$ and $\mathrm{K}_{\mathrm{L}}^{0}$. The mass splitting is an indicator of the degree to which FCNCs are permitted, since the mixing of states is suppressed by a cancellation between generations of quarks. If we take ETC as the cause of any splitting, we get a lower bound on $\Lambda_{\mathrm{ETC}}$ :

$$
\frac{\Delta m_{\mathrm{K}}}{m_{\mathrm{K}}} \sim \frac{f_{\mathrm{K}}^{2}}{2 \Lambda_{\mathrm{ETC}}^{2}},
$$

where $f_{\mathrm{K}} \approx 113 \mathrm{MeV}$ is the kaon decay constant [18, 48]. Experimentally the splitting is observed to be $\Delta m_{\mathrm{K}}=(3.484 \pm 0.006) \times 10^{-12}$, which puts the lower bound on $\Lambda_{\mathrm{ETC}}$ at

$$
\Lambda_{\mathrm{ETC}} \gtrsim 10^{3} \mathrm{TeV}
$$

The $\mathrm{K}^{0}$ meson consists of strange and anti strange, so $\overline{\mathrm{K}}^{0}-\mathrm{K}^{0}$ system places limits on generation of the strange quark mass; however, the most recent experimental data also allows a similar bound to arise for the $\overline{\mathrm{D}}^{0}-\mathrm{D}^{0}$ system. Since the $\mathrm{D}^{0}$ consists of charm and anticharm, the bound now applies to the generation of the charm mass; and as the charm is much heavier than the strange, the problem is enhanced [49]. 
This constrains the fermion masses to be of the order $1 \mathrm{keV}$, which is far smaller than any of the SM fermion masses save for the neutrinos. We have thus created a tension between two different observations: that the SM fermions have masses well above $1 \mathrm{keV}$, and that FCNCs are highly constrained by, for instance, the neutral kaon system; we would therefore like to find some way of decoupling the mechanisms controlling these two phenomena. This mechanism turns out to be one known as walking, introduced in [50-52], where the coupling runs very slowly in the region of a fixed point that is not quite reached. This allows the ETC scale (and hence the suppression of FCNCs) to be tuned independently of the SM fermion masses.

To see this, look back at equations (2.21) and (2.22). In the latter, we have calculated the chiral condensate at the TC scale; however, in fact we need to do so at the ETC scale; this adds a multiplicative correction dependent on the anomalous dimension $\gamma_{*}(g)$

$$
\langle\bar{\Psi} \Psi\rangle_{\mathrm{ETC}}=\langle\bar{\Psi} \Psi\rangle_{\mathrm{TC}} \exp \left[\int_{\Lambda_{\mathrm{TC}}}^{\Lambda_{\mathrm{ETC}}} \frac{d \mu}{\mu} \gamma_{*}(g(\mu))\right]
$$

Now, QCD-like theories have small $\gamma_{*}$, since their couplings run quickly to zero in the UV-leaving us with the same problems we had two paragraphs ago. However, we mentioned in the previous section for that theories at or near the lower edge of the conformal window, the anomalous dimension of the chiral condensate is $\gamma_{*} \approx 1$; if we look for a theory where the coupling is roughly constant (i.e. running very slowly, or "walking") between $\Lambda_{\mathrm{TC}}$ and $\Lambda_{\mathrm{ETC}}$, then $\gamma_{*}$ will be constant over this range, and (2.25) becomes

$$
\langle\bar{\Psi} \Psi\rangle_{\mathrm{ETC}}=\langle\bar{\Psi} \Psi\rangle_{\mathrm{TC}}\left(\frac{\Lambda_{\mathrm{ETC}}}{\Lambda_{\mathrm{TC}}}\right)^{\gamma_{*}}
$$

and we see that we have achieved our aim: while the FCNCs are still controlled by $\Lambda_{\mathrm{ETC}}$, the fermion mass is controlled by $\frac{\Lambda_{\mathrm{ETC}}}{\Lambda_{\mathrm{TC}}}$; by choosing a large $\Lambda_{\mathrm{ETC}}$ and a small $\Lambda_{\mathrm{TC}}$, we may satisfy both constraints.

Walking is conjectured to occur in theories lying immediately below the lower end of the conformal window (the same region as we expect $\gamma_{*} \approx 1$ ), indicated by the arrow in figure 2.2. As one can see in figure 2.1b, the infrared fixed point of the conformal theory is approached but not quite reached, leading to the walking behaviour. 


\section{THE STANDARD MODEL AND NOVEL STRONG INTERACTIONS}

\subsubsection{Techniscalars}

In contrast to the elementary Higgs theory, where EWSB leaves a single scalar state (the Higgs) behind, we have yet to see any light scalars emerge from Technicolor. We of course expect scalars from EWSB, however, in general there will be more than one unbroken generator, and these resulting scalars acquire a large mass from the $\alpha$ term in equation (2.20) - which is convenient, since we don't observe a large number of scalars in the physical spectrum. We do, however, observe one, which has been mentioned above and whose discovery was sought after with great enthusiasm: the boson found at the LHC which matches the expected properties of the Higgs.

One might reasonably ask, in the light of this, what reason we have to continue studying Technicolor; surely the discovery of a state not observed in its spectrum has put the nails in its coffin? Of course, theories are harder to kill than that, and there are a number of different explanations of states which could match the properties of the observed Higgs within the Technicolor framework, of which two of the most promising are technipions and technidilatons.

Firstly, consider a Technicolor theory where EWSB produces four scalars; there three are Higgsed into the gauge bosons, leaving a single scalar behind. The large mass enhancement mentioned previously is not compulsory, so the remaining technipion may become the light scalar observed experimentally. In principle, a theory giving a larger number of technipions could equally well have this property; however, only one such scalar has been observed. The discovery of a second Higgs-like scalar would be a strong indicator that the MSM was inadequate.

Secondly, consider again the Lagrangian of the MSM,

$$
\mathscr{L}_{\mathrm{SM}}=-\frac{1}{2} \operatorname{tr} F^{2}+\bar{\psi} i \not D \psi+(D H)^{\dagger} D H-Y \bar{\psi} H \psi-V\left(H^{\dagger} H\right),
$$

where the Higgs potential is now factorized as:

$$
V\left(H^{\dagger} H\right)=\lambda^{\prime}\left(H^{\dagger} H-\frac{v_{\mathrm{W}}^{2}}{2}\right)^{2} .
$$

In the limit $\lambda^{\prime}=0$, this is entirely scale-invariant; it is broken by the minimum of the Higgs potential at a scale $v_{\mathrm{W}}$. The breaking of this dilatation symmetry gives rise to a 
dilaton; in the case of the MSM this turns out to be the Higgs, acquiring a mass

$$
m_{\mathrm{h}}^{2}=2 \lambda^{\prime} v_{\mathrm{W}}^{2}
$$

Most of the calculations to study such a state (provided it is, as is required, light) can be carried out at tree level, with perturbation theory providing small corrections; thus any theory with a similar dilaton in its spectrum will have similar properties - not only quantum numbers, but also its couplings and phenomenology. The differences would appear at high energy and at high precision in the sub-leading corrections. However, since QCD does not, one might reasonably ask why we expect a Technicolor theory to provide us with a dilaton. One possible answer comes from the walking dynamics: we expect an IR near-fixed-point in the near-conformal theories of interest for walking behaviour; if the IR is sufficiently "near" conformality, then we would expect a dilaton to appear from the breaking of this symmetry. Recent work includes [53], in which the authors find a light scalar emerging from a string dual (although the theory falls short of being a Walking Technicolor candidate for other reasons), [54], where the authors show another gravity theory dual to a potential walking Technicolor theory with a light technidilaton, and [55], which again uses a gravity dual, showing that the technidilaton it admits is consistent with a physical mass of $125 \mathrm{GeV}$.

The most recent results from the LHC experiments [56] more firmly suggest that the observed state at $125 \mathrm{GeV}$ is a scalar rather than a pseudoscalar; if correct, this would eliminate technipions as a candidate explanation. Further experimental study of the state's properties is necessary to fully evaluate Technicolor as an explanation for EWSB.

\subsubsection{Areas of research}

Current research on Technicolor spans a vast area; relevant reviews summarising their findings, as well as going into background beyond the scope of this work, include $[1,57-$ $61]$.

As discussed in section 2.2.1, Dietrich and Sannino [37] narrowed the parameter space of available $\mathrm{SU}(N)$ theories somewhat; they showed that for $N_{\mathrm{f}}>1$ flavours in a representation $\mathrm{R}$, there is a limited set of $N_{\mathrm{f}}, \mathrm{R}$ which have asymptotic freedom: for the fundamental, adjoint, and two-index symmetric and antisymmetric representations, all 


\section{THE STANDARD MODEL AND NOVEL STRONG INTERACTIONS}

$N$ are allowed, and for $N \leq 9$ there are a few other special cases, namely the three-index antisymmetric representation for $N_{\mathrm{f}}=2,6 \leq N \leq 9$, and the four-index antisymmetric representation for $N_{\mathrm{f}}=2, N=8$. If one allows for $N_{\mathrm{f}} \geq 1$ flavours, then the set of exceptions grows, but still only the fundamental, adjoint, and two-index symmetric and antisymmetric representations remain for $N \geq 16$.

The methods in use complement each other: field theoretical methods, such as Seiberg duality [62] and the analyses quoted in section 2.2.2, are semi-analytic. In the case of section 2.2.2, an uncontrolled approximation is made. It is sufficient to show that the various regions seen exist; however, the boundaries it gives on these regions are by no means precise. Other techniques are needed to look non-perturbatively and in a more controlled manner; two such techniques are holography and the lattice.

The holographic method divides into bottom-up approaches, where the $4 \mathrm{D}$ theory has an additional dimension added, working in the direction of finding a gravity dual with as little intervention as possible, and top-down approaches, where one works from full string theory to find the properties of the Technicolor theory. The latter - in the case of Technicolor, rather than in the wider field where the opposite holds - is the more recent, powerful, and difficult approach. Recent bottom-up results include [53, 55, 6374], whilst recent phenomenological findings of the top-down approach include [75-81], and recent theoretical developments include [82-87]. Holography captures the broad features of the theories it looks at well, but is a large- $N$ method, meaning it becomes less trustworthy at smaller $N$.

In order to verify the results from the large- $N$ approach at small $N$, and to explore theories that cannot be reached holographically, we look to the best known firstprinciples technique: the lattice, where the space-time is quantised to a discrete lattice. This approach is the method used for the work in this thesis, and is described in the next chapter, with results from the literature quoted afterwards.

\subsection{Conclusions}

In this chapter I have outlined in brief the important features of Technicolor, in particular those that put the rest of this project in context. Technicolor offers a neat alternative to the elementary Higgs for EWSB, using phenomena already demonstrable in $\mathrm{QCD}$, and by extending it with an additional gauge boson and allowing it to walk 
we find that it explains problems that the Standard Model Higgs fails to explain. I have also mentioned alternative explanations for the observation of a scalar at $125 \mathrm{GeV}$ at the LHC.

Some examples have been quoted from the literature of theories that have been studied analytically in perturbation theory using the gauge-gravity duality approach. However, analytic technology is insufficient to probe many aspects of the theory, specifically non-perturbative effects at finite number of colors. For this reason we need a fully first-principles, non-perturbative method for studying theories; to this end in the next chapter we introduce the lattice, to discretise the theory and analyse it numerically. 
2. THE STANDARD MODEL AND NOVEL STRONG INTERACTIONS 


\section{Chapter 3}

\section{Gauge Theories on the Lattice}

In this chapter, I will motivate and describe the set of techniques known as Lattice Gauge Theory (LGT). Since a complete treatment could easily fill multiple textbooks, the scope of this chapter will be limited to considering those techniques used in carrying out the work described in chapters 4-6. In section 3.1 I will describe the necessity for LGT to investigate strongly-coupled non-Abelian gauge theories, and describe its basic principles. In section 3.2, I will describe the algorithms and specific techniques used for the computations carried out for this project (excluding those developed specifically to deal with the theory discussed in chapter 4 , and those looking at topological observables, which are treated separately in chapter 5). Then in sections 3.3 and 3.4, I will look at existing lattice results in the literature, both for QCD and for candidate Technicolor theories, and outline the two theories that will be studied in this work, respectively.

\subsection{Motivation and Approach}

Computations in gauge theory require calculations of functional integrals across field space, which are analytically tractable only in special cases. Around these cases, we may use perturbation theory when there is a suitable small parameter to expand in (i.e. at weak coupling). Additionally, the functional integrals found in gauge theory diverge, and must be regulated (and renormalised) for results to be meaningful.

Approaching a problem that is not analytically tractable, an instinct that has existed since the time of Newton is to discretise the problem: what was a continuum problem then becomes a discrete problem. In the case of lattice gauge theory, the four- 


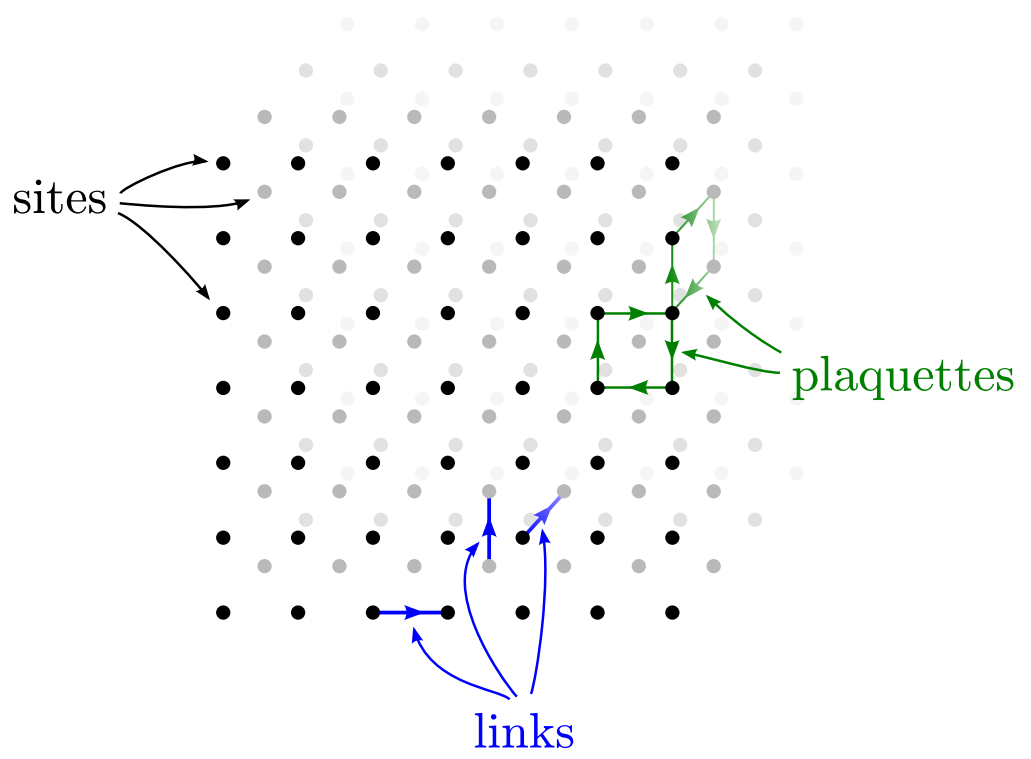

Figure 3.1: A three-dimensional example of a lattice setup. Lattice sites are in black; in blue are links which connect adjacent sites; the closed green paths are plaquettes.

dimensional space-time is discretised into a lattice (a term borrowed from condensed matter theory, since many early techniques were inspired by and borrowed from the techniques for looking at atomic lattices).

Since high energy is equivalent to small wavelength, this has the secondary effect of acting as a regulator: wavelengths smaller than the lattice spacing (traditionally referred to as $a$ ) will not be represented, giving an energy cut-off $\Lambda$ which rises as the lattice spacing is decreased. Since the aim of any discrete calculation is to be able to approximate to the "true" case that would be found in the absence of discretisation (the continuum limit), we will always aim to take (or at least estimate) the limit $a \rightarrow 0$, i.e. $\Lambda \rightarrow \infty$, as must be done with a regulator.

The lattice is Wick-rotated to have imaginary time - that is, it is a Euclidean lattice, with all-positive metric. The reasoning for this will become clear below. The space-time is generally given boundary conditions that are either periodic or antiperiodic (where fermionic variables change sign across the boundary); the calculations for the work of this thesis use periodic boundary conditions in the spatial directions and antiperiodic in time. (One can also use other boundary conditions such as twisted periodic; these are outside the scope of this work and will not be discussed further.) 
A three-dimensional example of such a space-time lattice is shown in figure 3.1. As one might expect from a discretised space-time, the lattice consists of a regular array of points, referred to as sites, and labeled with a position vector. In principle this can be any regular pattern, but in practise most simulations have a hypercubic lattice ${ }^{1}$ (although studies at high temperature use an "anisotropic" lattice with a different spacing in the temporal direction). Fermion fields have values defined on each lattice site. Running between adjacent lattice sites are links, which are labeled by the position of the site on which they start, and an index denoting their direction. The gauge fields live on the links, and are represented as link variables, which are exponentiations of the components of the gauge potential at the start of the link:

$$
U_{\mu}(\mathbf{x})=e^{i g a A_{\mu}(\mathbf{x})}
$$

Only positive directions are considered for link variables; the equivalent in the reverse direction may be obtained by taking the hermitian conjugate of the variable on the desired link. The smallest square that can be defined on the lattice, from four adjacent lattice sites, is referred to as the plaquette, and the path-ordered product of link variables around the plaquette is the plaquette variable (which is also frequently referred to as a plaquette). It shares the letter $U$ with the link variable, but carries two indices denoting its orientation. The plaquette variable is used to form the gauge action, being as it is the exponentiation of the field strength tensor.

In principle the lattice can be used to perform analytical calculations and form new perturbative approximations, and much early work was done this way; for example, the strong coupling expansion uses the lattice formulation to give insights that could not be previously seen. But as in the continuum if not moreso, this approach is limited to a handful of special cases. The discrete lattice, however, like most discretised problems, lends itself to being probed using computers. The most naïve approaches to discretisation, with finite difference iteration methods, break down rapidly as the problem size increases, making them unsuitable for even the smallest lattice problems; statistical methods must instead be used. Even with these methods, calculations will exhaust the highest-performance machines that can be sourced; a large zoology of algorithms has

\footnotetext{
${ }^{1}$ Hypercubic here is used in the crystallographic sense-i.e., the unit cell is a hypercube, with the lattice sites lying on it corners. The extent of the lattice is thus a hypercuboid; as is mentioned later, in this work the volume is $L^{3} \times 2 L$.
} 


\section{GAUGE THEORIES ON THE LATTICE}

been developed over the years to eke the maximum performance out of the machines that can be afforded; the ones used in this project are described in section 3.2.

Most current algorithms make use of importance sampling; this is where the Euclidean lattice becomes invaluable: rather than the path integral including a factor $e^{i S}$, it instead has a factor $e^{-S}$, thus provided the action is real and positive definite (which will be the case for this project), the path integral has a weight function allowing importance sampling to be used.

The gauge action takes the form [88]:

$$
S_{\mathrm{g}}=\beta \sum_{p}\left(1-\frac{1}{N} \operatorname{Re} \operatorname{tr} U(p)\right)
$$

with $N$ that of the $\mathrm{SU}(N)$ gauge group, and the sum over $p$ representing all plaquettesimplying a sum over orientations and sites.

Much has been written in the literature about the various methods of putting fermions on the lattice. In the most naïve discretisation, the problem that is encountered is that for each fermion field added, an additional 15 "doublers" also appear. Later analysis by Nielsen and Ninomiya showed that in fact the absence of doublers and preservation of chiral symmetry in the simulation are mutually exclusive [89], given the constraint that ultralocality is preserved (i.e. that interactions only couple neighbours at finite distance). The formulation used in this work is the Wilson formulation [90], whose (fermionic) action takes the form

$$
S_{\mathrm{F}}=\sum_{x, y} \bar{\psi}(x) D(x, y) \psi(y)
$$

where the discretised Dirac operator $D(x, y)$ has the form

$$
D(x, y)=\delta_{x, y}-\frac{\kappa}{2}\left[\left(1-\gamma_{\mu}\right) U_{\mu}(x) \delta_{y, x+\mu}+\left(1+\gamma_{\mu}\right) U_{\mu}^{\dagger}(x-\mu) \delta_{y, x-\mu}\right] .
$$

$\kappa$ here is referred to as the hopping parameter, and is related to the bare fermion mass; $\beta$ is related to the gauge coupling (and controls the lattice spacing)

$$
\begin{aligned}
\kappa & =\frac{1}{8+2 m} \\
\beta & =\frac{2 N}{g^{2}} .
\end{aligned}
$$


This explicitly breaks chiral symmetry in the action, removing all doublers in the continuum limit; chiral symmetry can also be restored (again in the continuum limit) by tuning the fermion mass. This method was chosen due to its ease of use and its maturity, as well as its ability to eliminate all doublers. (An alternative approach known as staggered fermions, introduced by Kogut and Susskind in [91], keeps three doublers, but also preserves some chiral symmetry [92, 93]. Non-ultralocal choices are also possible, but become very difficult to simulate, due to their high computational cost.)

A lattice computation can be broken down into three sections, each requiring different algorithms.

- Firstly, a statistical ensemble of gauge field configurations is generated using an appropriate algorithm. Since anticommuting numbers cannot be stored on a computer, fermion fields are integrated out, leaving a fermionic determinant. Calculation of this is computationally expensive, especially close to the chiral limit, and so historically this was sometimes set to 1 , in what is called the quenched approximation. The physical interpretation of this approximation is that contributions from sea quarks are ignored. Advances in computing power mean that this approximation is rarely used now without another reason. When the full determinant is calculated, the fermions are referred to as dynamical; all of the work in this project uses dynamical fermions. For most physics of interest, this stage requires either a cluster or a supercomputer.

- Once an ensemble of gauge fields is prepared, observables are calculated by taking appropriate correlation functions on the gauge field background. By analogy with experimental physics, where the background of reality is taken and observations are made, the calculation of observables are frequently referred to as "measurements". This stage also requires a considerable amount of computing power, but generally less than the generation of configurations.

- With the output from calculations of correlation functions in hand, the data must then be analysed to give meaningful results for the observables of interest. This is the least computationally demanding aspect, and can usually be reasonably performed on a workstation, or on the front-end node of a HPC facility. 


\section{GAUGE THEORIES ON THE LATTICE}

Methods used for all three aspects are described in the next section.

In QCD, research collaborations frequently specialise in either the generation of configurations (and performing a small number of measurements to demonstrate their utility), or in performing measurements on configurations generated by other collaborations. This is due to the high demands on compute power and on algorithm development for both sides, and also due to the large number of groups working on lattice QCD: reusing configurations avoids reduplication of effort. Where lattice gauge theory is used as a tool outside of QCD, for example in BSM research, collaborations more often generate configurations and then perform measurements of interest on them (although the configurations can obviously then be reused for other purposes). The techniques used therefore generally lag those in lattice QCD a little: techniques are developed and proved by specialists working on QCD, and those that prove themselves are adopted by the wider community. However, there are also some specific techniques that are developed mainly to study the physics of theories showing (near-)conformal behaviour. The work described in chapter 4 includes generation of gauge configurations, measurement of observables, and analysis of the resulting data, while that in chapter 5 makes use of configurations previously generated on another related project.

\subsection{Algorithms}

\subsubsection{RHMC algorithm}

The Rational Hybrid Monte Carlo (RHMC) algorithm (first described in 1998 by Kennedy, Horváth, and Sint [94], and summarised nicely by Clark [95]) is an extension of the Hybrid Monte Carlo (HMC) algorithm [96-98], allowing for a non-even number of dynamical fermions. The HMC algorithm itself is a combination of three other algorithms: the Langevin algorithm [99-101], based on Markov chains, the Molecular Dynamics (MD) algorithm [102, 103], which time-evolves configurations in a deterministic manner (when combined, these two methods become the Hybrid Molecular Dynamics algorithm), and the Metropolis algorithm [104], which ensures a correctlysampled distribution by introducing an accept-reject step.

Starting with the lattice path integral for Wilson fermions:

$$
\langle O\rangle=\frac{1}{Z} \int[d U] e^{-S_{\mathrm{g}}(U)}[\operatorname{det} \mathscr{M}(U)]^{N_{\mathrm{f}} / 2} O(U),
$$


where $\mathscr{M}=M^{\dagger} M$, and $M$ is the discretised Dirac operator, we then (taking $N_{\mathrm{f}}=2$ for simplicity) rewrite the fermionic determinant in terms of pseudofermions (bosonic fields capturing the fermionic dynamics):

$$
\operatorname{det} \mathscr{M}=\int D \phi^{\dagger} D \phi e^{-\phi^{\dagger} \mathscr{M}^{-1} \phi}=\int D \phi^{\dagger} D \phi e^{-S_{\mathrm{f}}} .
$$

The steps the HMC algorithm takes to update a configuration are then:

1. The momentum heat bath, where random conjugate momenta for the gauge field are chosen as $P(\pi) \propto e^{-\pi^{*} \pi / 2}$,

2. The pseudofermion heat bath, where pseudofermion fields are randomly chosen as $\phi \propto D \xi ; \xi$ is a random noise field, $P(\xi)=e^{-\xi^{*} \xi}$,

3. The Molecular Dynamics trajectory, where the link variables and momenta are evolved in an appropriate deterministic way,

4. Accept or discard the new configuration with an appropriate probability.

In the case of the RHMC algorithm, however, we keep $N_{\mathrm{f}}$ arbitrary, and make a rational approximation for the negative power of the fermion matrix:

$$
\operatorname{det} \mathscr{M}=\int D \phi^{\dagger} D \phi e^{-\phi^{\dagger} \mathscr{M}^{-N_{\mathrm{f}} / 2} \phi}=\int D \phi^{\dagger} D \phi e^{-\phi^{\dagger} r^{2}(\mathscr{M}) \phi},
$$

where $r(x)=x^{-N_{\mathrm{f}} / 4}$. Step 2 then generates fields chosen as $\phi \propto r(\mathscr{M})^{-1} \xi$. Suitable rational approximations may be generated by the Remez algorithm [105]. We then proceed as we would with the HMC.

Since the fermionic determinant contains the square of the rational function, evaluating the evolution equations for the MD trajectory in step 3 would cause a double inversion; this is avoided by using an altered rational approximation for the MD: $\bar{r} \approx \mathscr{M}^{-N_{\mathrm{f}} / 2} \approx r^{2}$. The pseudofermionic contribution then becomes a sum of HMC-like terms:

$$
S_{\mathrm{pf}}^{\prime}=-\sum_{i=1}^{m} \alpha_{i} \phi^{\dagger}\left(\mathscr{M}+\beta_{i}\right)^{-1} \mathscr{M}^{\prime}\left(\mathscr{M}+\beta_{i}\right)^{-1} \phi
$$




\section{GAUGE THEORIES ON THE LATTICE}

\subsubsection{Meson masses}

Masses of states are found by calculating the correlation functions of appropriate quantities. These quantities take the form $O^{\dagger}(x) O(y)$, where $O$ is an operator carrying the quantum numbers of the state in question; in the case of mesons, this is

$$
O(x)=\bar{\psi}_{a}(x) \Gamma \psi_{b}(x)
$$

where $a, b$ refer to fermion flavours, and $\Gamma$ is the combination of $\gamma$ matrices carrying appropriate quantum numbers. The correlation functions are then written in the pathintegral formulation as:

$$
C(x, y)=\frac{\int D U D \bar{\psi} D \psi O^{\dagger}(x) O(y) e^{-S}}{\int D U D \bar{\psi} D \psi e^{-S}}=\frac{I(x, y)}{Z}
$$

where $Z$ refers to the partition function, by analogy with statistical mechanics.

Since fermions are Grassman-valued fields, they can’t be represented directly numerically, so we must integrate them out by hand. We use Wick's theorem for this, where each fermion contraction gives a factor of the fermion propagator. If we mark a contraction as $\bar{\psi}_{a} \psi_{b}$, then we see that there is one set of contractions available for $a \neq b$, but two for $a=b$. In the first case, we have

$$
I(x, y)=\int D U D \bar{\psi} D \psi \bar{\psi}_{b} \bar{\Gamma} \bar{\psi}_{a}(x) \bar{\psi}_{a} \Gamma \psi_{b}(y) e^{-S}
$$

where $\bar{\Gamma}=\gamma_{0} \Gamma^{\dagger} \gamma_{0}$ has been introduced (simplifying some other algebra that has been done), since the propagator from a fermion of one species to one of another is zero. This case is referred to as a flavour non-singlet (and is often called a triplet, from the isospin triplet that appears in QCD). In the second case, the flavour singlet, however, we instead have

$$
I(x, y)=\int D U D \bar{\psi} D \psi \bar{\psi}_{a} \bar{\Gamma} \bar{\psi}_{a}(x) \bar{\psi}_{a} \Gamma \psi_{a}(y) e^{-S}
$$

where the two sets of contractions are added together. The upper contraction is the same in both the $a=b$ and $a \neq b$ cases; when the contractions are replaced with propagators, this gives a term of the form $-\operatorname{tr} \bar{\Gamma} D^{-1}(x ; y) \Gamma D^{-1}(y ; x)$, whose calculation 
is well-explored and is described in the next section. The lower contraction, however, which is unique to the $a=b$ case, takes the form $\operatorname{tr} \bar{\Gamma} D^{-1}(x ; x) \Gamma D^{-1}(y ; y)$ : here, the propagators lie on the diagonals of the inverse Dirac operator, and represent disconnected (or "hairpin") terms. The calculation of disconnected diagrams is harder than connected terms, since large cancellations mean that statistical noise in the terms being subtracted overwhelms any signal. For this reason, lattice QCD has historically focused on states accessible with only triplet diagrams; it is only recently that advances in algorithms and compute power have allowed the disconnected diagrams contributing to singlet states to be calculated, and such calculations still require more specialist techniques and greater care than triplet calculations. Some of these techniques are described in section 3.2.2.2.

\subsubsection{Triplet masses - the Z2SEMWall algorithm}

Whilst the previous section mentions the ease of calculation of triplet masses, this ease is relative, and some care is still necessary to obtain results with good precision in a practicable amount of time. The Dirac operator is too large and sparse to invert efficiently and in reasonable time in its entirety; instead, the inverse is only calculated in the case of its application to a small set of fields, referred to as source fields. The result of the application is then referred to as a sink field. For a given source field $\eta$, the corresponding sink field $\psi$ may be found as

$$
\psi(\mathbf{y}, t)=\sum_{\substack{\text { spin } \\ \text { color } \\ \mathbf{x}, t}} D^{-1}(\mathbf{y}, t ; \mathbf{x}, \tau) \eta(\mathbf{x}, \tau)
$$

The simplest and most common way of implementing this is using point sources; that is, sources which take a value of 1 at one point in parameter space and 0 everywhere else: $\eta_{A \alpha}^{(B \beta)}=\delta_{A B} \delta_{\alpha \beta} \delta_{\mathbf{x y}} \delta_{\tau t_{0}}$. Here $A, \alpha$ are color and spin indices, but $B, \beta$ are labels distinguishing between different source fields. To incorporate this into an algorithm, 


\section{GAUGE THEORIES ON THE LATTICE}

we rearrange to find an expression for $D^{-1}$, by substituting into (3.15):

$$
\begin{aligned}
\psi_{A \alpha}^{(B \beta)} & =\sum_{\mathbf{x}, \tau, C, \gamma} D_{A \alpha, C \gamma}^{-1}(\mathbf{y}, t ; \mathbf{x}, \tau) \eta_{C \gamma}^{(B \beta)}(\mathbf{x}, \tau) \\
& =\sum_{\mathbf{x}, \tau, C, \gamma} D_{A \alpha, C \gamma}^{-1}(\mathbf{y}, t ; \mathbf{x}, \tau) \delta_{A B} \delta_{\alpha \beta} \delta_{\mathbf{x y}} \delta_{\tau t_{0}} \\
& =D_{A \alpha, C \gamma}^{-1}\left(\mathbf{y}, t ; \mathbf{x}_{0}, t_{0}\right) .
\end{aligned}
$$

Thus any occurrence of $D^{-1}$ may be replaced by a combination of two sink fields, one to and one from $\left(\mathbf{x}_{0}, t_{0}\right)$. However, this product introduces a sensitivity to local fluctuations in the gauge field configuration, which means more configurations must be sampled to give good statistics.

This problem may be overcome by use of stochastic sources [106-108] (also known as the Stochastic Estimator Technique), which are defined probabilistically rather than absolutely as was the case for the point sources. To illustrate this we look at the example of Z2SEMWall sources.

First consider the quantity $Z_{2}(\mathbf{x}, t) \in\left\{\frac{ \pm 1 \pm i}{\sqrt{2}}\right\}$, with uniformly and independently distributed random values on each point in the parameter space. Then

$$
\left\langle Z_{2}(\mathbf{x}, A) Z_{2}^{*}(\mathbf{y}, B)\right\rangle=\delta_{A B} \delta \mathbf{x y}
$$

If we now defined the Z2SEMWall source (with Z2 referring to the $Z_{2}$ quantity mentioned above, SEM referring to the Spin Explicit method-i.e. one generator per spin component - and Wall referring to the presence of the source on a single timeslice-i.e. a 3D "wall" on the $4 \mathrm{D}$ lattice) as:

$$
\eta_{A \alpha}^{(\beta)}=\delta_{\alpha \beta} \delta_{t t_{0}} Z_{2}(\mathbf{x}, A)
$$

where $\beta$ is a label running from 1 to 4 , and $A, \alpha$ are color and spin indices respectively, then we can find the expectation value of:

$$
\sum_{\gamma}\left\langle\eta_{A \alpha}^{(\gamma)} \eta_{B \beta}^{(\gamma) *}\right\rangle=\delta_{A B} \delta_{\alpha \beta} \delta_{\mathbf{x y}} \delta_{t t_{0}} \delta_{s s_{0}}
$$

Incorporating the Z2SEMWall source into an algorithm requires a little more work 
than the point source. If we wish to calculate a correlation function of the form:

$$
\begin{aligned}
C & =\left\langle\left(\bar{\psi}_{1} \Gamma \psi_{2}\right)^{\dagger}(x) \bar{\psi}_{1} \Gamma \psi_{2}(0)\right\rangle \\
& =-\frac{1}{V_{3}} \sum_{\mathbf{x}} \operatorname{tr} \bar{\Gamma} D^{-1}(\mathbf{x}, t ; \mathbf{0}, 0) \Gamma D^{-1}(\mathbf{0}, 0 ; \mathbf{x}, t)
\end{aligned}
$$

where $V_{3}=L^{3}$ is the spatial lattice volume. Since $\mathbf{x}$ is a dummy variable, we can rename it at will, and we also introduce a second summation variable $\mathbf{y}$, which introduces another factor of $V_{3}\left(\sum_{\mathbf{y}} 1=V_{3}\right)$

$$
\begin{aligned}
C & =-\frac{1}{V_{3}^{2}} \sum_{\mathbf{z y}} \operatorname{tr} \bar{\Gamma} D^{-1}(\mathbf{z}, t ; \mathbf{0}, 0) \Gamma D^{-1}(\mathbf{0}, 0 ; \mathbf{z}, t) \\
& =-\frac{1}{V_{3}^{2}} \sum_{\mathbf{x y}} \operatorname{tr} \bar{\Gamma} D^{-1}(\mathbf{x}-\mathbf{y}, t ; \mathbf{0}, 0) \Gamma D^{-1}(\mathbf{0}, 0 ; \mathbf{x}-\mathbf{y}, t)
\end{aligned}
$$

where in the second line we have substituted $\mathbf{z}=\mathbf{x}-\mathbf{y}$. Now, making use of the relation $D^{-1}=\gamma_{5} D^{-1 \dagger} \gamma_{5}$, the cyclicity of the trace operator, and the boundary conditions, this may be reexpressed as:

$$
\begin{aligned}
& C=-\frac{1}{V_{3}^{2}} \sum_{\mathbf{x y}} \operatorname{tr} \gamma_{5} \bar{\Gamma} D^{-1}\left(\mathbf{x}, t+t_{0} ; \mathbf{y}, t_{0}\right) \Gamma \gamma_{5} D^{-1}\left(\mathbf{y}, t_{0} ; \mathbf{x}, t+t_{0}\right)^{\dagger} \\
&=-\frac{1}{V_{3}^{2}} \sum_{\mathbf{x y}} \operatorname{tr}\left(\gamma_{5} \bar{\Gamma}\right)_{\alpha \beta} D_{A \beta, B \gamma}^{-1}\left(\mathbf{x}, t+t_{0} ; \mathbf{y}, t_{0}\right)\left(\Gamma \gamma_{5}\right)_{\gamma \delta} D_{A \alpha, B \delta}^{-1}\left(\mathbf{x}, t+t_{0} ; \mathbf{y}, t_{0}\right)^{*} \\
&=-\frac{1}{V_{3}^{2}} \sum_{\mathbf{x y} \cdots} \operatorname{tr}\left(\gamma_{5} \bar{\Gamma}\right)_{\alpha \beta} D_{A \beta, B \gamma}^{-1}\left(\mathbf{x}, t+t_{0} ; \mathbf{y}, t_{1}\right) \delta_{\gamma \gamma^{\prime}} \delta_{B B^{\prime}} \delta_{\mathbf{y y}^{\prime}} \delta_{t_{1} t_{0}} \delta_{t_{2} t_{0}} \times \\
&\left(\Gamma \gamma_{5}\right)_{\gamma^{\prime} \delta} D_{A \alpha, B^{\prime} \delta}^{-1}\left(\mathbf{x}, t+t_{0} ; \mathbf{y}^{\prime}, t_{2}\right)^{*}
\end{aligned}
$$

The delta functions have been introduced so that we can now replace them with the 


\section{GAUGE THEORIES ON THE LATTICE}

stochastic sources, which have the same behaviour under the summation over $\mathbf{y}$ :

$$
\begin{aligned}
& C=-\frac{1}{V_{3}^{2}} \sum_{\mathbf{x y} \cdots} \operatorname{tr}\left(\gamma_{5} \bar{\Gamma}\right)_{\alpha \beta} D_{A \beta, B \gamma}^{-1}\left(\mathbf{x}, t+t_{0} ; \mathbf{y}, t_{0}\right) \eta_{B \gamma}^{(\omega)}\left(\mathbf{y}, t_{1}\right) \times \\
& \eta_{B^{\prime} \gamma^{\prime}}^{(\omega)}\left(\mathbf{y}^{\prime}, t_{2}\right)\left(\Gamma \gamma_{5}\right)_{\gamma \delta} D_{A \alpha, B \delta}^{-1}\left(\mathbf{x}, t+t_{0} ; \mathbf{y}, t_{0}\right)^{*} \\
& =-\frac{1}{V_{3}^{3}} \sum_{\mathbf{x y} \cdots}\left[D_{A \alpha, B^{\prime} \delta}^{-1}\left(\mathbf{x}, t+t_{0} ; \mathbf{y}^{\prime}, t_{2}\right)\left[\left(\Gamma \gamma_{5}\right)^{\dagger}\right]_{\delta \gamma^{\prime}} \eta_{B^{\prime} \gamma^{\prime}}^{(\omega)}\left(\mathbf{y}^{\prime}, t_{2}\right)\right]^{*} \times \\
& {\left[\left(\gamma_{5} \bar{\Gamma}\right)_{\alpha \beta} D_{A \beta, B \gamma}^{-1}\left(\mathbf{x}, t+t_{0} ; \mathbf{y}, t_{1}\right) \eta_{B \gamma}^{(\omega)}\left(\mathbf{y}, t_{1}\right)\right]} \\
& =-\frac{1}{V_{3}^{3}} \sum_{\mathbf{x y} \omega}\left[D^{-1}\left(\Gamma \gamma_{5}\right)^{\dagger} \eta^{(\omega)}\right]^{\dagger} \gamma_{5} \bar{\Gamma} D^{-1} \eta^{(\omega)}\left(\mathbf{x}, t+t_{0}\right)
\end{aligned}
$$

where we have now removed the dummy co-ordinate fields again. Now we can substitute the sink fields back in:

$$
C=-\frac{1}{V_{3}^{3}} \sum_{\mathbf{x y} \omega} \psi_{\Gamma}^{(\omega) \dagger}\left(\mathbf{x}, t+t_{0}\right) \gamma_{5} \bar{\Gamma} \psi^{(\omega)}\left(\mathbf{x}, t+t_{0}\right)
$$

where the set of sink fields is

$$
\psi^{(\omega)}(\mathbf{x}, t)=D^{-1} \eta^{(\omega)}(\mathbf{x}, t), \quad \psi_{\Gamma}^{(\omega)}(\mathbf{x}, t)=D^{-1}\left(\Gamma \gamma_{5}\right)^{\dagger} \eta^{(\omega)}(\mathbf{x}, t)
$$

Here, $\psi_{\gamma_{5}}^{(\omega)}=\psi^{(\omega)}$. In the case of point sources, $4 d_{\mathrm{R}}$ sink fields (and therefore inversions) were required; here, $4 N_{\text {channels }}$ are required, where $N_{\text {channels }}$ is the number of different $\Gamma$ s that are used (which must include $\gamma_{5}$ ). For SU(3) and 16 channels, 32 inversions would be necessary for point sources, but 64 would be necessary for Z2SEMWall sources. For SU(2) on the other hand, point sources would only require 12 inversions, but Z2SEMWall sources would still require 64. This ratio does not, however, correlate directly to the required computing time for a given precision, since point sources require more configurations to be sampled, increasing the computing time requirement.

\subsubsection{Singlet Hairpins}

Disconnected diagrams, as was mentioned previously, are problematic due to a cancellation of two relatively noisy terms, where the noise is larger than the difference. The techniques to overcome this, therefore, comprise a variety of individual methods to 
reduce noise in such calculations, described for instance in $[109,110]$. In this project, the Truncated Solver Method (TSM) $[109,111]$ was used, as well as stochastic sources.

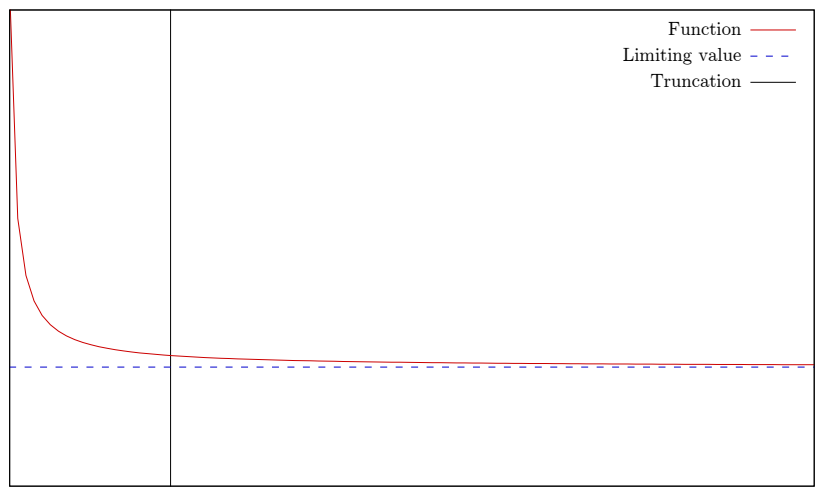

Figure 3.2: A sketch illustrating the truncation principle. The function of interest has a long tail before it "truly" reaches its asymptote, but rapidly reaches within $5 \%$ of it (in less than a fifth of the time); computations thus could be spared by computing to equilibrium enough to characterise a truncation length and correction, and subsequently truncating the measurements.

The truncated solver method is based on the knowledge that while the convergence of an observable to its "true" value becomes slower as it becomes close, it does so in a consistent manner (as can be seen in figure 3.2). Thus one can use a small number of expensive "pilot" measurements, letting them fully converge, and then use their behaviour to calculate two parameters: the optimum point at which to truncate the observation, and the correction that must be made from the truncated value to the estimated true value. With that in place, a larger number of truncated estimates can be made in a much smaller time, giving rise to greatly improved statistics.

\subsubsection{Glueballs}

Glueballs are bound states of pure glue. Their masses are found in a similar way to mesons; however, since they have no fermion content, there is no requirement to integrate out the fermion fields (and no fermionic determinant to calculate), and so the operators consist only of products of traced Wilson loops. However, since the glueball is an extended object, individual plaquettes (and operators constructed of a small number of them) have a very small overlap with the state of interest, and the signal becomes drowned by noise from contamination by other states. 


\section{GAUGE THEORIES ON THE LATTICE}

This problem is overcome by smearing and blocking of the gauge links forming the operators. The link smearing used in this work is based on the APE smearing method $[112,113]$, and is described in $[114,115]$; in brief, to each link, the nearest, and next-tonearest, parallel-transported neighbours are added, with appropriately-chosen weight factors. A more helpful diagrammatic explanation is given in figure 3.3. The blocking procedure, as described in $[116,117]$, forms a blocked link of length $2 a$ by taking the direct path, and adding to it the spatial "staples" - that is, routes of length $4 a$ via the nearest parallel transported direct paths. Another useful graphical representation is shown in figure 3.4 .

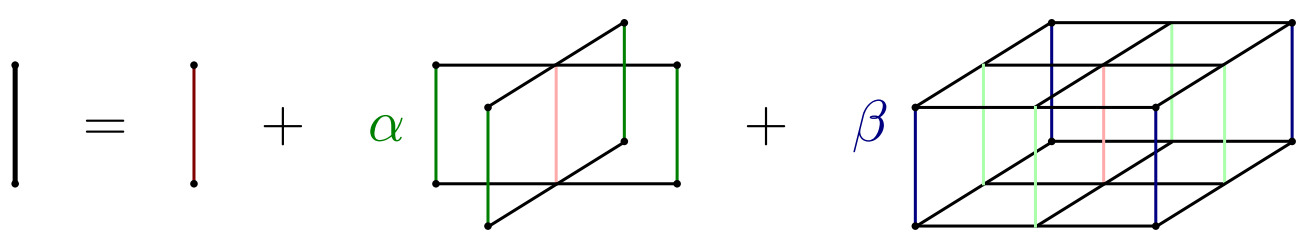

$(2)$

(3)

Figure 3.3: Smeared links are found by adding the original link (labeled as (1)) to the parallel transported nearest neighbours (labeled as (2)), weighted by a factor of $\alpha$, and the next-to-nearest-neighbours (labeled as (3)), weighted by a factor of $\beta$.

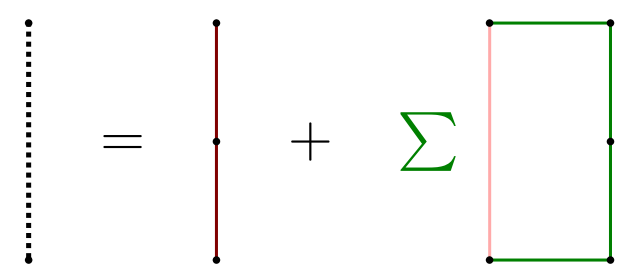

$(1)$

$(2)$

Figure 3.4: Blocked links are found by summing spatial "staples" and adding the result to the direct (straight line) path; this procedure is then iterated.

\subsubsection{Anomalous Dimension}

The anomalous dimension of a theory with a conformal fixed point, characterising as it does the scaling behaviour of the observables in the theory, should be visible from fitting of any of the quantities in the theory. However, there are some observables that allow easier observation than others, and in particular a method recently described by 
Patella [118] allows a much preciser determination of $\gamma_{*}$ than was previously possible. This method makes use of the spectrum of eigenvalues of the Dirac operator, and is the method used to determine $\gamma_{*}$ for the theory discussed in chapter 4 . The basis of the method is the observation that the spectral density $\rho$ of the Dirac operator is related to the eigenvalue $\omega$ by a power law for small eigenvalues; to leading order this is

$$
\rho(\omega)=\hat{\rho}_{0} \mu^{\frac{4 \gamma_{*}}{1+\gamma_{*}}} \omega^{\frac{3-\gamma_{*}}{1+\gamma_{*}}}+\ldots,
$$

where $\hat{\rho}_{0}$ is a dimensionless constant, and $\mu$ is the renormalisation scale. In the case of a mass-deformed theory, this relation becomes valid only in an intermediate range of eigenvalues.

Consider the positive definite operator defined by

$$
M=(\not D+m)^{\dagger}(\not D+m)=m^{2}-\not D^{2} .
$$

Then define the mode number per unit volume $\bar{\nu}(\Omega)$ as the number of eigenvalues of $M$ below the cutoff $\Omega^{2}$, divided by the volume

$$
\bar{\nu}(\Omega)=\int_{-\sqrt{\Omega^{2}-m^{2}}}^{\sqrt{\Omega^{2}-m^{2}}} \rho(\omega) d \omega=2 \int_{0}^{\sqrt{\Omega^{2}-m^{2}}} \rho(\omega) d \omega .
$$

For the intermediate region of interest in a mass-deformed theory, we cut off the lower end and get

$$
\bar{\nu}(\Omega)=\bar{\nu}_{0}(m)+2 \int_{\bar{\omega}_{\mathrm{IR}}}^{\sqrt{\Omega^{2}-m^{2}}} \rho(\omega) d \omega,
$$

where $\bar{\nu}_{0}(m)$ is a constant to be determined. $\rho(\omega)$ in the second equation can be replaced by equation (3.37), giving

$$
\bar{\nu}(\Omega)=\bar{\nu}_{0}(m)+\frac{1}{2}\left(1+\gamma_{*}\right) \hat{\rho}_{0} \mu^{\frac{4 \gamma_{*}}{1+\gamma_{*}}}\left(\Omega^{2}-m^{2}\right)^{\frac{2}{1+\gamma_{*}}} .
$$

$\bar{\nu}_{0}(m)$ depends on the fermion mass, but in a predictable manner: since $\bar{\nu}(\Omega)$ is renormalisation group invariant (per [119]), and has dimensions [mass] $]^{4}$, then

$$
\bar{\nu}_{0}(m) \propto m_{\mathrm{PS}}^{4},
$$

where $m_{\mathrm{PS}}$ is the mass of the pseudoscalar meson (although any other particle mass 


\section{GAUGE THEORIES ON THE LATTICE}

could be used; the PS is chosen due to its general ease of access).

To relate equation (3.41) to lattice output, it must be reexpressed in terms of bare lattice quantities rather than continuum renormalised ones, this gives

$$
a^{-4} \bar{\nu}(\Omega) \approx a^{-4} \bar{\nu}_{0}(m)+A\left[(a \Omega)^{2}-(a m)^{2}\right]^{\frac{2}{1+\gamma_{*}}}
$$

where $A$ is a dimensionless constant, and $m$ is proportional to the PCAC mass.

For the purposes of this work, the method of projectors is used, as per [119]. A projector $\mathbb{P}(\Omega)$ is defined over the eigenspaces of $M$ corresponding to eigenvalues below $\Omega^{2}$. Armed with this, the mode number per unit volume becomes

$$
\bar{\nu}(\Omega)=\lim _{V \rightarrow \infty} \frac{1}{V}\langle\operatorname{tr} \mathbb{P}(\Omega)\rangle
$$

The projector is approximated using an appropriate rational function

$$
\mathbb{P}(\Omega) \approx h(\mathbb{X})^{4} ; \quad \mathbb{X}=1-\frac{2 \Omega_{*}^{2}}{M+\Omega_{*}^{2}},
$$

where $h(x)$ is a polynomial approximating $\theta(-x)$ in the range $\sqrt{\epsilon} \leq|x| \leq 1$ (and $\epsilon$ here characterises the precision of the approximation, and conversely the degree of the polynomial), and $\Omega_{*}$ is a parameter of order $\Omega$, defined as

$$
\frac{\Omega}{\Omega_{*}}=\left(\frac{1-\sqrt{\epsilon}}{1+\sqrt{\epsilon}}\right)^{\frac{1}{2}}+\int_{-\sqrt{\epsilon}}^{\sqrt{\epsilon}} d x \frac{1+x}{\left(1-x^{2}\right)^{3 / 2}} h(x)^{4} .
$$

The trace may be estimated stochastically, and the error due to making the polynomial approximation for the projector may be estimated from the fit of the spectral density.

\subsubsection{Wilson and Polyakov Loops}

The static potential for a fermion and antifermion may be calculated using correlators of either Wilson or Polyakov loops. Historically the Wilson loop method was developed first and so is more widely used in the literature; however, the Polyakov loop method is simpler to perform and gives improved results. The Polyakov loop method is mostly used in this work, with the Wilson loop approach being used as a check of the consistency in a selection of cases. In addition, histograms of Polyakov loops allow the status of the centre symmetry to be checked. 


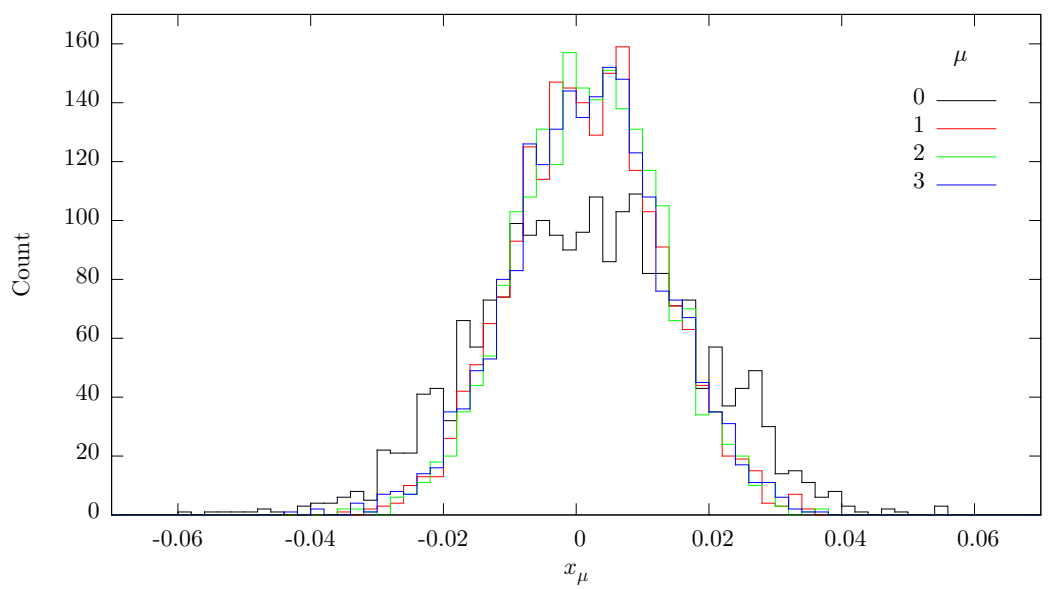

Figure 3.5: The histogram of the Polyakov loop of one of the data sets studied in chapter 4 ; the single peak seen shows that it is centre symmetric.

The Polyakov loop is the product of gauge links in a single direction, closed due to the (anti)periodic boundaries of the space. In the $\hat{\rho}$ direction, the Polyakov loop is given by

$$
\bar{P}_{\rho}=\sum_{\{x \mid \rho=0} \prod_{n=0}^{N_{\rho}-1} U(x+n \rho, \hat{\rho}),
$$

where the sum runs over the lattice slice normal to $\hat{\rho}$. $\mathrm{SU}(N)$ theories have a $\mathbb{Z}_{N}$ centre symmetry, so in the case of $\mathrm{SU}(2)$ we expect a $\mathbb{Z}_{2}$ symmetry, which we expect the Polyakov loop to obey. Thus it is trivial to see whether or not the centre symmetry is broken: if it is preserved, then the average Polyakov loop is always consistent with zero (and the system is confined); if not, then it acquires a value of $\pm a$, where $a$ is some constant (and the system has become deconfined). However, since the average Polyakov loop may be stable at $+a$ for some number of configurations, but then switch to $-a$, and back again, the average over all configurations may still be consistent with zero. We therefore look at the histogram of the average Polyakov loop, considering each spatial and time dimension separately. In the centre symmetric phase, we will see a single-peaked distribution about 0 ; in the centre broken phase, we see a double-peaked distribution at $\pm a$. An example of such a histogram is shown in figure 3.5.

Consider a loop in the $x_{0}$ direction, at a fixed $x_{1}$, and define the operator $P_{0}\left(x_{1}\right)$ 


\section{GAUGE THEORIES ON THE LATTICE}

to be the average in the other two directions

$$
P_{0}\left(x_{1}\right)=\frac{1}{N_{\mathrm{s}}^{2}} \sum_{x_{2}, x_{3}} \frac{1}{N} \operatorname{tr}\left(\prod_{n_{0}=0}^{N_{\mathrm{t}}-1} U_{0}\left(a n_{0}, \mathbf{r}\right)\right)
$$

where $\mathbf{r}$ is the 3 -vector $\left(x_{1}, x_{2}, x_{3}\right), N_{\mathrm{s}}, N_{\mathrm{t}}$ are the number of sites in the spatial and temporal directions respectively, and $N$ is that of $\mathrm{SU}(N)$. If we then take a vacuumsubtracted correlator, we see

$$
\left\langle P_{0}\left(x_{1}\right)^{\dagger} P_{0}\left(x_{1}+X_{1}\right)\right\rangle-\left|\left\langle P_{0}\left(x_{1}\right)\right\rangle\right|^{2}=\sum_{n=0}^{\infty}\left|c_{n}\left(a N_{\mathrm{t}}\right)\right|^{2} e^{-E_{n}\left(a N_{\mathrm{t}}\right) X_{1}}, \quad E_{n}>0 \forall n,
$$

where $c_{n}$ is the overlap $c_{n}=\left\langle 0\left|P_{0}\left(x_{1}\right)\right| n\right\rangle$, and $|n\rangle$ is the $n$th eigenstate of the Hamiltonian. Then for large $X_{1}$, the exponential decays leaving the ground state energy of the fundamental string $E_{0}\left(a N_{\mathrm{t}}\right)$. In the case of a confining theory in $4 \mathrm{D}$, this should fit

$$
E_{0}\left(a N_{\mathrm{t}}\right)=\sigma a N_{\mathrm{t}}-\frac{c \pi}{3 a N_{\mathrm{t}}}
$$

up to subleading corrections, where $\sigma$ is the temporal fundamental string tension. We can define similar operators $P_{i}$ wrapping the spatial directions, and by fitting their correlators in the temporal direction obtain the spatial string tension. At zero temperature in the confined phase, in which the theory has an unbroken centre symmetry, then the spatial and temporal string tensions should be in agreement. As is the case with glueballs, these basic Polyakov operators have a low overlap with the physics of interest; the solution to this is again to smear the links.

The Wilson loop, meanwhile, is a product of gauge links, again around a closed loop, but now closed within the lattice rather than winding around it. Although in general a Wilson loop can refer to any such closed loop, in the context of this work we restrict ourselves to rectangular Wilson loops of spatial extent $R$ and temporal extent $T$. We may interpret this as an infinitely-heavy (i.e. static) fermion-antifermion pair being created at a distance $R$ apart, and annihilating a time $T$ later.

For convenience, we can decompose the loop into spatial and temporal components, 
as

$W(T, \mathbf{R})=\operatorname{tr}\left\{U_{\mathrm{t}}[t \rightarrow t+T ; \mathbf{r}]^{\dagger} U_{\mathrm{s}}[t ; \mathbf{r} \rightarrow \mathbf{r}+\mathbf{R}] U_{\mathrm{t}}[t \rightarrow t+T ; \mathbf{r}+\mathbf{R}] U_{\mathrm{s}}[t+T ; \mathbf{r} \rightarrow \mathbf{r}+\mathbf{R}]^{\dagger}\right\}$

Since $\mathbf{R}$ needn't be on-axis (and indeed allowing it to be off-axis allows a larger range of $R$ values to be probed), the procedure of [120] is used. In the Hamiltonian gauge, the expectation value $\langle W(T, \mathbf{R})\rangle$ becomes the time-correlator for the operator creating a heavy fermion-antifermion pair connected by a string, $M(\mathbf{R})=\bar{\psi}(\mathbf{r}) U_{\mathrm{s}}[\mathbf{r} \rightarrow \mathbf{r}+\mathbf{R}] \psi(\mathbf{r}+$ $\mathbf{R})$,

$$
\begin{aligned}
\langle W(T, \mathbf{R})\rangle & =\frac{1}{Z\left(a N_{\mathrm{t}}\right)} \operatorname{tr}\left[e^{-\left(a N_{\mathrm{t}}-T\right) H} M(\mathbf{R})^{\dagger} e^{-T H} M(\mathbf{R})\right] \\
& =\frac{1}{Z\left(a N_{\mathrm{t}}\right)} \sum_{n m}|\langle n, \mathbf{R}|M(\mathbf{R})| m\rangle|^{2} e^{-a N_{\mathrm{t}} E_{m}} e^{-T\left[V_{n}(R)-E_{m}\right]}
\end{aligned}
$$

where $|m\rangle$ are the vacuum eigenstates of energy $E_{m}$, and $|n, \mathbf{R}\rangle$ are the states with energy $V_{n}(R)$ in the presence of the fermion-antifermion pair. The lowest-lying such energy $V_{0}(R)$ is the static potential.

To extract the string tension, we look in an intermediate region, since at $T$ near 0 and near $a N_{\mathrm{t}}$, one of the sets of states collapses to a single state. In the intermediary region we expect to see a good fit to a straight line, with the gradient being the string tension, as was the case in the Polyakov loop calculation.

Once again, smearing is used to improve the statistics; ${ }^{1}$ in this case, however, the full variational procedure is not applied. Instead, the full variational procedure is applied only to a small (but well-decorellated) sample of configurations, to find the smearing parameters that give the optimal results for the reduced ensemble. These parameters are then taken as the smearing values for the full ensemble. While not as effective as the full variational method, this approximation gives a significant improvement in computation time, allowing the analysis on more modest hardware than would otherwise be required. While the smearing will distort the potential at small $R$, and add a constant additive factor, neither of these affects our fit for $\sigma$, since we are looking at gradients in the intermediate- $R$ region.

\footnotetext{
${ }^{1}$ For the Wilson loops, HYP smearing (introduced in [121]), a method based on and ultimately similar to the previously-discussed APE smearing, is used.
} 


\section{GAUGE THEORIES ON THE LATTICE}

\subsubsection{Extracting Results}

For most observables, the output from the measurement code is a set of correlation functions, one per measurement per configuration analysed (although some codes will make multiple measurements of the same observable on the same gauge configuration, since the gauge configuration contains more data than is used by the single "hit").

For a state with a given set of quantum numbers, an operator carrying the same quantum numbers is constructed; let us say that this is called $O$. The correlation function that is calculated by the code then takes the form

$$
\left\langle O^{\dagger}(\mathbf{x}, 0) O(\mathbf{y}, t)\right\rangle=\int \mathcal{D} U \mathcal{D} \psi \mathcal{D} \bar{\psi} O^{\dagger}(\mathbf{x}, 0) O(\mathbf{y}, t) e^{-S[U, \psi, \bar{\psi}]}
$$

where the state has been allowed to move in space and time. We can take a time evolution factor $e^{-H t}$ out, and by inserting complete sets of states, reduce the righthand side to

$$
\left\langle O^{\dagger}(\mathbf{x}, 0) O(\mathbf{y}, t)\right\rangle=\sum_{n} \alpha_{n} e^{-E_{n} t}
$$

Taking the long-time limit of this, we see

$$
\left\langle O^{\dagger}(\mathbf{x}, 0) O(\mathbf{y}, t)\right\rangle \stackrel{t \rightarrow \infty}{\longrightarrow} \alpha_{0} e^{-m t}
$$

On a finite lattice, we unfortunately do not have the luxury of taking an infinitetime limit; however, in many cases it is still possible to extract a reasonable estimate for the mass, as the decay is rapid enough to see the ground state emerge in finite time. To extract the mass, firstly an effective mass plot is produced from the average correlation function. The effective mass is the mass that is found if the limiting value of the correlation function is taken to be at the current data point. (In the case of mesonic correlation functions, the data are first "folded over" in the temporal direction since the correlation function is symmetric in this direction.) This plot will obviously tend towards the limiting value of the mass in the long-time limit; thus if a stabilisation (or plateau) is observed, then that is taken to be the limiting value. Averages are then taken over the respective ranges identified as being part of the plateaux. An example of an effective mass plot with plateau marked is shown in figure 3.6.

In the simplest case one can use an arithmetic mean and bootstrap sampling [122124] — where random samples are taken from the data and averaged (or fitted), with 


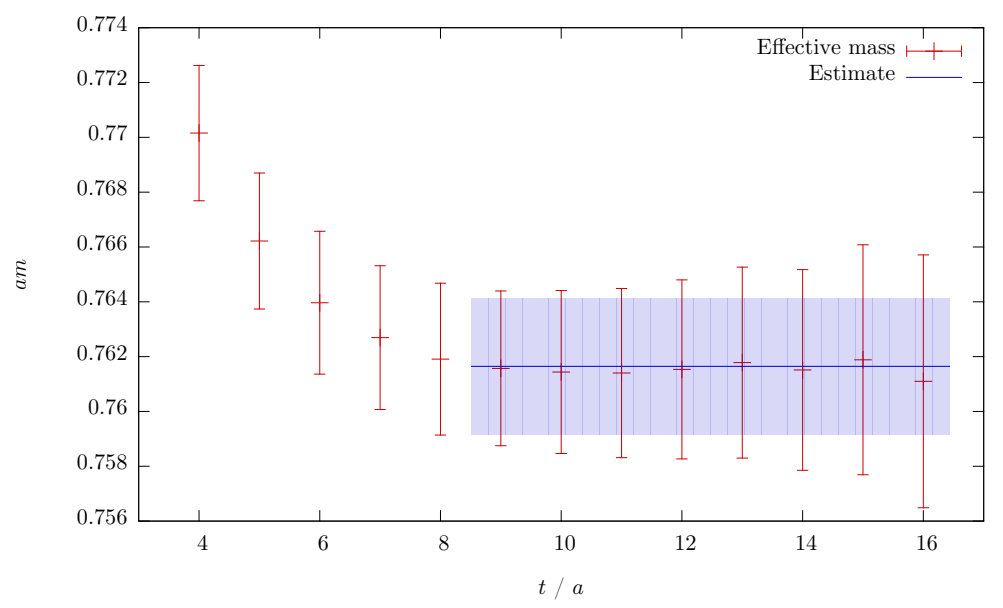

Figure 3.6: An effective mass plot for a meson mass; the plateau in this case was identified between $t=9$ and $t=16$.

the set of sample averages giving a new data set - to give an estimate of the statistical error for the masses. However, there are more sophisticated techniques that can be used to give tighter bounds. For instance, for calculating meson masses, the noise in the correlation function tends to dominate in the centre of the lattice; thus if the bootstrap efficiency - the proportion of the bootstrap samples taken which can be successfully fitted - is below $100 \%$, then the noisy middle can be slowly removed from the sample until the efficiency reaches $100 \%$. This avoids both the bias that would be introduced by allowing a sub-100\% efficiency, and the additional computer time that would be necessary to improve the sample size to give $100 \%$ efficiency with all points included.

\subsection{Existing Lattice Studies}

To put the numerical study performed in this work into a broader context, in this section I will briefly review the state of the art of lattice simulations, beginning with the more familiar example of QCD.

\subsubsection{QCD}

Pure QCD studies are sufficiently numerous that even beginning to enumerate them would be impracticable; fortunately there exist studies dedicated to collating results from other studies in lattice QCD. Lattice QCD gives the correct spectrum for mesonic 


\section{GAUGE THEORIES ON THE LATTICE}

states, including many states which were predicted by the lattice results before they were observed experimentally (at the predicted masses); results for the light mesons from [3] are shown in figure 3.7, and of the results from [4] in figure 3.8. Similarly the baryon spectrum, illustrated in figure 3.9 from [5], also agrees with experiment. Lattice results have become the accepted values for some quantities, or make a significant contribution to them. For example, the strong coupling strength $\alpha_{\mathrm{s}}$, shown in figure 3.10 as collated in [6], has its most precise estimate from lattice results, and the estimate is found to be in good agreement with all experimental methods. The CKM matrix can also be calculated on the lattice (reviewed in [125]), and shows good agreement in all but one element; work is ongoing to ascertain why that is the case.

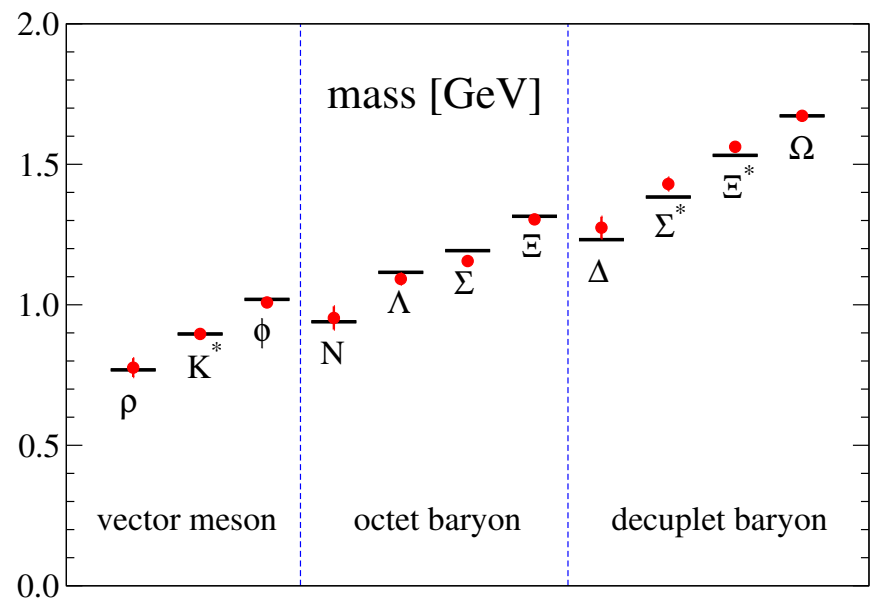

Figure 3.7: The light meson spectrum of lattice QCD, as of 2008, from [3].

\subsubsection{Technicolor}

Since Dietrich and Sannino's perturbative exploration of where we expect to see conformal and near-conformal theories that was reviewed in chapter 2 [37], a number of lattice groups have taken an interest into testing their predictions. There are two main areas of focus: the first at theories with fundamental quarks (as in QCD), where large $N_{\mathrm{f}}$ is needed to approach the conformal window; this is done using the staggered fermion approach, since it lends itself naturally to large $N_{\mathrm{f}}$. The second approach is to explore higher representations, where fewer flavours are needed to approach the conformal window. 


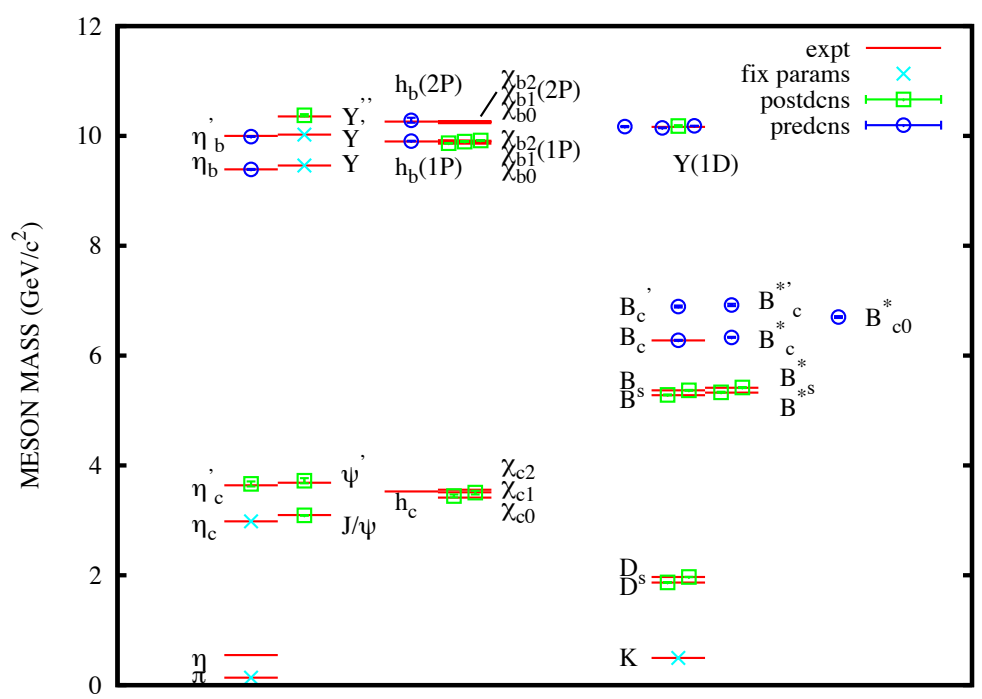

Figure 3.8: The ("gold-plated") heavy meson spectrum of lattice QCD, as of 2012, from [4].

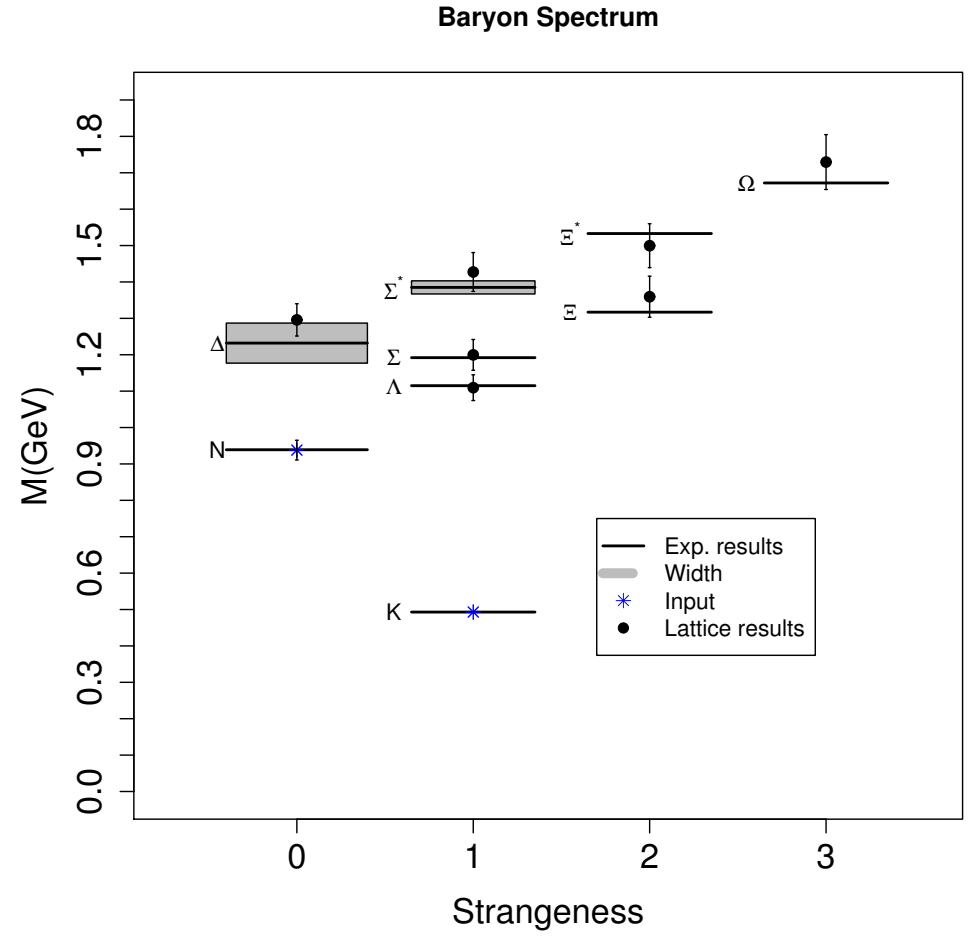

Figure 3.9: The baryon spectrum from lattice QCD, as of 2009, from [5]. 


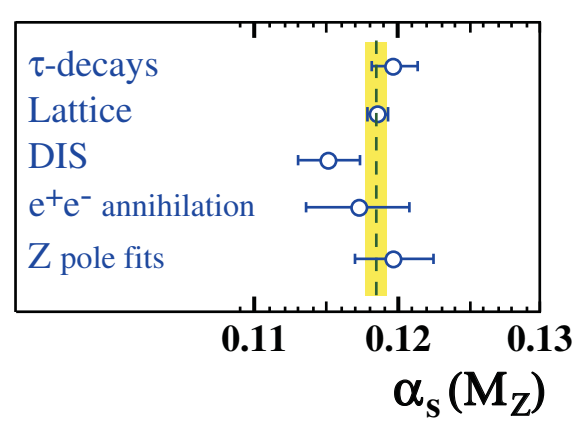

Figure 3.10: Comparison of various experimental methods with the lattice result for $\alpha_{\mathrm{s}}$, as of 2012, from [6].

Recent results of both approaches are reviewed in [7], with a helpful diagram (reproduced in figure 3.11) summarising the findings; the theories with the most data are $\mathrm{SU}(3)$ theories with (unsurprisingly, given the limitations of the staggered approach) multiples of 4 flavours. The 4- and 8-flavour cases are found to be confining [126-132], and the 16-flavour case conformal [127, 133-136], while the 12-flavour case is marginal [126, 130, 135-137], either lying just within the conformal window or just below its lower edge. For $\mathrm{SU}(2)$ with fundamental flavours, the data is more sparse, but shows 8 and 10-flavour theories are conformal [138-140] the 2-flavour case is confining [141], and the cases in between are marginal [139, 140, 142-144]. For the higher representation, meanwhile, studies have been inconclusive for the SU(3) [145-153] and SU(4) [147, 154] theories with symmetric fermions, while for $\mathrm{SU}(2)$ with adjoint fermions a number of studies indicate that $N_{\mathrm{f}}=2$ is conformal. This theory will be elaborated upon more in the next section.

Current work is looking to further pin down the lower end of the conformal window, and also to look for walking signals in theories which are believed to be near-conformal (for example, [155] shows signs that 8-flavour fundamental SU(3) may be walking).

\subsection{Theories Under Investigation}

The two theories under investigation in this work are both $\mathrm{SU}(2)$ theories with adjoint fermions. The adjoint representation of $\mathrm{SU}(2)$ is real, leading $\psi_{\mathrm{L}}$ and $\bar{\psi}_{\mathrm{R}}$ to both have left chirality, and to transform under the same representation of the gauge group. This allows the combination of the fields into a single multiplet; if chiral symmetry breaks, 


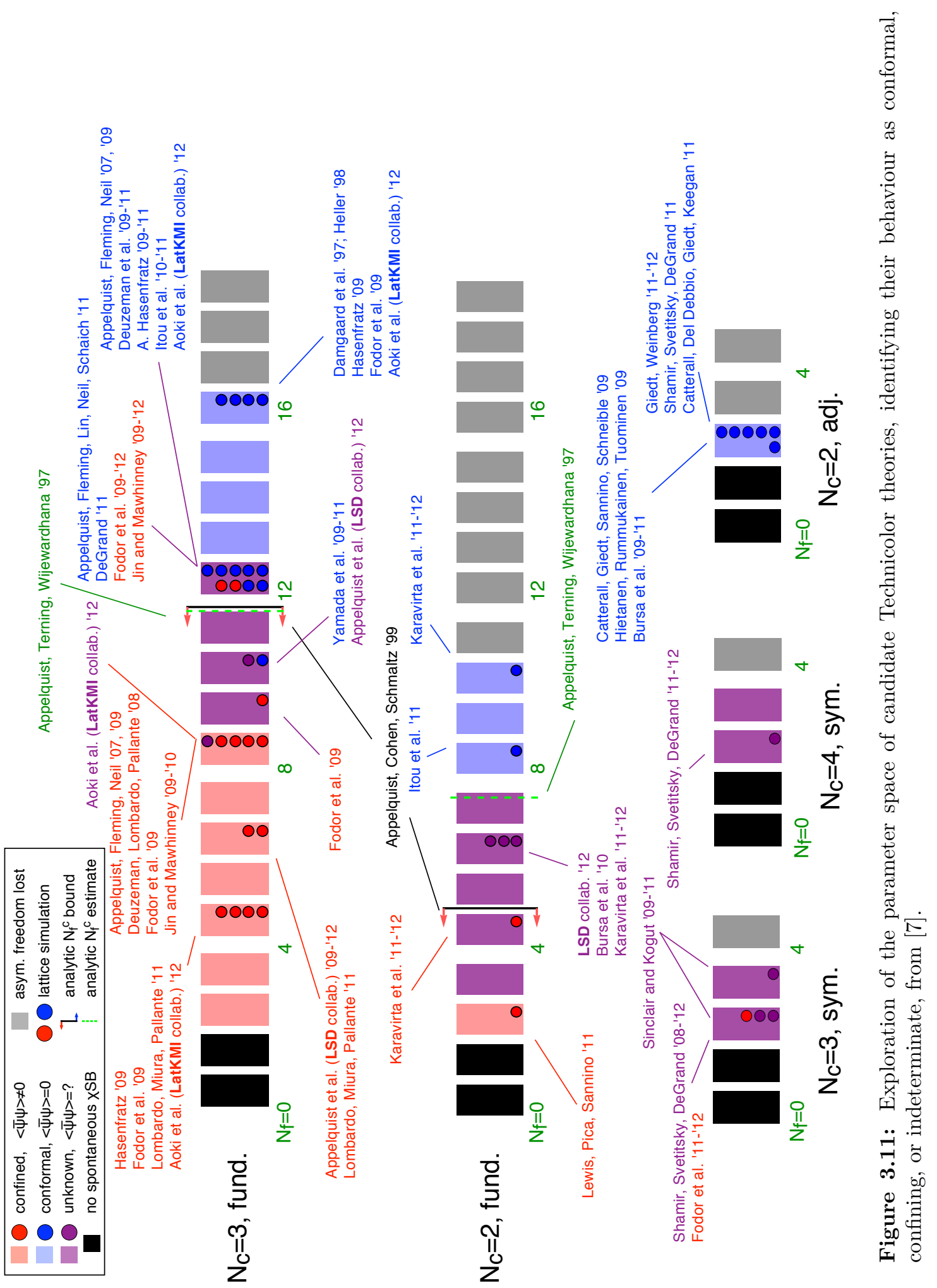




\section{GAUGE THEORIES ON THE LATTICE}

this gives a breaking pattern of

$$
\mathrm{SU}\left(2 N_{\mathrm{f}}\right) \rightarrow \mathrm{SO}\left(2 N_{\mathrm{f}}\right)
$$

leading to the production of $\left(N_{\mathrm{f}}+1\right)\left(2 N_{\mathrm{f}}-1\right)$ Goldstone bosons, of which three (if available) are eaten by $\mathrm{W}^{ \pm}$and $\mathrm{Z}$.

From the discussion in the previous chapter (in particular equations (2.13) and (2.19)), the analysis of Dietrich and Sannino [37] places perturbative bounds on the conformal window. For the adjoint representation, the Casimir $\left(2 N^{2}\right)$ and group dimension $\left(N^{2}-1\right)$ factors cancel individually, leaving the bounds fixed for all $N$. As the energy scale is lowered, the upper end is found at $N_{\mathrm{f}}^{\mathrm{I}}[\mathrm{R}]=2 \frac{3}{4}$, while the lower end (after which chiral symmetry breaks before the fixed point is reached) is at $N_{\mathrm{f}}^{\mathrm{II}}[\mathrm{R}]=2 \frac{3}{40}$, and the fixed point no is no longer found below $N_{\mathrm{f}}^{\mathrm{III}}[\mathrm{R}]=1 \frac{1}{16}$.

\subsubsection{Minimal Walking Technicolor}

$\mathrm{SU}(2)$ with two adjoint Dirac flavours is referred to as Minimal Walking Technicolor (MWT); the limits above suggest that it should be near-conformal. However, more recent lattice results [156] suggest that it is in fact IR-conformal; this is not completely unexpected, since the methods used in [37] were inexact, relying on the two-loop beta function. The name MWT has stuck, however. A study of its spectrum [156] shows scaling of its observables: mass ratios remain approximately constant through the range studied. (This range is approximately a factor of 6 in the quark mass.) This means that unlike in a confining theory, the pion mass cannot become parametrically small, and the pion a Goldstone of the theory, since its mass is constant relative to other masses in the theory.

Previous lattice studies such as [156] have looked at the spectroscopy of the theory and the running of the coupling. In chapter 5 I will present an investigation of the topology of this theory on the lattice, showing results that are consistent with the existing observation of IR-conformality - specifically, the topology is found not to differ from that of pure gauge $\mathrm{SU}(2)$. A fit for the anomalous dimension $\gamma_{*}$ is attempted but is unsuccessful.

A later lattice study of the theory [157] reveals that smaller lattice volumes simulated are affected by finite-size artefacts. The conformal nature of the theory makes 
such effects particularly severe, and so their identification is an important step in any lattice computation in a conformal or near-conformal theory. In the case of [157], the effects were revealed by checking consistency with larger lattices, and by using wallsmeared sources, which showed that the seemingly consistent results were an artefact of two effects cancelling each other. It is found that the instanton size distribution may be used as an additional indicator of finite-size effects.

\subsubsection{SU(2)Adj Nf1}

The theory that forms the main bulk of the work of this thesis is $\mathrm{SU}(2)$ with one adjoint Dirac flavour, for which I will use the name SU(2)Adj Nf1. Its chiral symmetry breaking $(\chi \mathrm{SB})$ produces only two Goldstone bosons; this is insufficient to give mass to the $\mathrm{W}^{ \pm}$and $\mathrm{Z}$ bosons. However, it is still worthy of study: while the 2-loop perturbative bounds suggest that the theory should be confining, since MWT has been found to be IR-conformal, knowing the IR behaviour of SU(2)Adj Nf1 will help to pin down the lower end of the conformal window non-perturbatively. It also allows development of a number of methods which may be applicable to other walking theories.

In chapter 4 a first look at this theory on the lattice will be presented, including studies of the meson and glueball spectrum, the string tension from Wilson and Polyakov loop correlators, and the anomalous dimension from the Dirac operator eigenvalue distribution. In chapter 5, the topology of the theory is considered and compared with that of MWT and pure-gauge SU(2). Although the results of [37] show this theory to not have an IRFP, the results found are tentatively indicative of a theory that is IR-conformal or near-conformal; the difference in IR behaviour between the analytical study and the lattice simulation is most likely again due to the approximate nature of Dietrich and Sannino's approach. 
3. GAUGE THEORIES ON THE LATTICE 


\section{Chapter 4}

\section{SU(2) with 1 Dirac (2 Majorana) Flavours}

In this chapter we will look at the results of an exploratory study of the $\mathrm{SU}(2)$ theory with one adjoint Dirac flavour (SU(2)Adj Nf1). By a change of basis, we will show in section 4.1.1 how this can also be viewed as the same theory but with two Majorana flavours. Spectroscopic results are presented in section 4.2, while topological observables are deferred until the next chapter.

\subsection{Methodology}

The spectroscopy of this theory is most readily understood in terms of Majorana flavours (of which, from degrees of freedom arguments, there are two); however, most existing lattice code (including HiRep, discussed in chapter 6) are designed to model Dirac fermions. This leaves us with a choice: we may spend a lot of time adapting the code to work with Majorana fields (along with the requisite debugging and testing), or we may adapt our requirements so that we can find properties of Majorana fields in terms of Dirac equivalents, so that our existing code can be used unmodified, with only the interpretation of its output being altered. For this work it was decided that the latter approach would be less time-consuming, and so in this section I will show the change of basis that was found. 


\section{SU(2) WITH 1 DIRAC (2 MAJORANA) FLAVOURS}

\subsubsection{Majorana decomposition}

Before moving on to the decomposition proper, let us first fix the notation for this section. We choose to work in the chiral representation of the Dirac algebra, for which

$$
\gamma^{\mu}=\left(\begin{array}{cc}
0 & \bar{\sigma}_{\mu} \\
\sigma_{\mu} & 0
\end{array}\right)
$$

where

$$
\sigma^{\mu}=(1, \boldsymbol{\sigma}), \quad \bar{\sigma}^{\mu}=(1,-\boldsymbol{\sigma}) .
$$

$\boldsymbol{\sigma}$ is the 3-vector formed from the Pauli matrices:

$$
\boldsymbol{\sigma}=\left(\sigma_{1}, \sigma_{2}, \sigma_{3}\right) ; \quad \sigma_{1}=\left(\begin{array}{cc}
0 & 1 \\
1 & 0
\end{array}\right), \sigma_{2}=\left(\begin{array}{cc}
0 & -i \\
i & 0
\end{array}\right), \sigma_{3}=\left(\begin{array}{cc}
1 & 0 \\
0 & -1
\end{array}\right)
$$

It is convenient to now define

$$
\gamma_{5}=i \gamma^{0} \gamma^{1} \gamma^{2} \gamma^{3}=\left(\begin{array}{cc}
1 & 0 \\
0 & -1
\end{array}\right)
$$

Spinors may be charge conjugated by

$$
\psi_{\mathrm{C}}=C \bar{\psi}^{\mathrm{T}}=C_{0} \psi^{*}
$$

where

$$
C=-i \gamma^{2} \gamma^{0}=\left(\begin{array}{cc}
i \sigma_{2} & 0 \\
0 & -i \sigma_{2}
\end{array}\right), C_{0}=-i \gamma^{2}=\left(\begin{array}{cc}
0 & i \sigma_{2} \\
-i \sigma_{2} & 0
\end{array}\right)
$$

Majorana spinors are the subset of spinors which are invariant under charge conjugation; i.e.

$$
\psi_{\mathrm{MC}}=\psi_{\mathrm{M}}
$$

They have half the degrees of freedom of a Dirac spinor.

Now, for the previously-promised decomposition, consider the two fermions

$$
\psi_{\mathrm{M}+}=\frac{\psi+C \bar{\psi}^{\mathrm{T}}}{2} \quad \psi_{\mathrm{M}-}=\frac{\psi-C \bar{\psi}^{\mathrm{T}}}{2 i},
$$

which obviously combine to form the Dirac spinor

$$
\psi=\psi_{\mathrm{M}+}+i \psi_{\mathrm{M}-} .
$$


This decomposition requires that $\psi$ and its charge-conjugate lie in the same representation of the gauge group, thus is only valid in real (or pseudoreal) representations; while the adjoint representation of $\mathrm{SU}(N)$ is permitted, the fundamental is not, so QCD with Majorana quarks could not be explored with this method. It is applicable for any $N$, and any number of Dirac flavours; it is currently unclear if or how it could extend to odd numbers of Majorana flavours, or whether that would introduce a sign problem.

To verify that these are Majorana spinors, we can charge-conjugate them:

$$
\begin{aligned}
& \bar{\psi}_{\mathrm{M}+}=\frac{\bar{\psi}+\psi^{\mathrm{T}} C}{2} \Rightarrow C \bar{\psi}_{\mathrm{M}+}^{\mathrm{T}}=\frac{\psi+C \bar{\psi}^{\mathrm{T}}}{2}=\psi_{\mathrm{M}+} \\
& \bar{\psi}_{\mathrm{M}-}=\frac{\psi^{\mathrm{T}} C-\bar{\psi}}{2 i} \Rightarrow C \bar{\psi}_{\mathrm{M}-}^{\mathrm{T}}=\frac{\psi-C \bar{\psi}^{\mathrm{T}}}{2 i}=\psi_{\mathrm{M}-}
\end{aligned}
$$

i.e. the charge conjugates of $\psi_{\mathrm{M} \pm}$ are $\psi_{\mathrm{M} \pm}$, so $\psi_{\mathrm{M} \pm}$ are indeed Majorana spinors.

\subsubsection{Action and $\chi \mathrm{SB}$}

The fermionic action of the one-flavour theory takes the form

$$
S_{\mathrm{f}}=\bar{\psi} \not \partial \psi+m \bar{\psi} \psi
$$

Armed with the reexpression from the previous section, we would like to find an equivalent link between the Dirac and Majorana actions

$$
\begin{aligned}
S_{\text {Dirac }} & \leftrightarrow S_{\text {Majorana }} \\
\bar{\psi} \not \partial \psi+m \bar{\psi} \psi & \leftrightarrow \bar{\psi}_{\mathrm{M}+} \not \partial \psi_{\mathrm{M}+}+m \bar{\psi}_{\mathrm{M}+} \psi_{\mathrm{M}+}+\bar{\psi}_{\mathrm{M}-} \not \partial \psi_{\mathrm{M}-}+m \bar{\psi}_{\mathrm{M}-} \psi_{\mathrm{M}-} \cdot
\end{aligned}
$$

Looking at the mass term first, we see

$$
\begin{aligned}
& \bar{\psi}_{\mathrm{M}+} \psi_{\mathrm{M}+}=\frac{1}{4}\left[\bar{\psi} \psi+\psi^{\mathrm{T}} C \psi+\bar{\psi} C \bar{\psi}^{\mathrm{T}}+\psi^{\mathrm{T}} C C \bar{\psi}^{\mathrm{T}}\right]=\frac{1}{4}\left[2 \bar{\psi} \psi+\psi^{\mathrm{T}} C \psi+\bar{\psi} C \bar{\psi}^{\mathrm{T}}\right] \\
& \bar{\psi}_{\mathrm{M}-} \psi_{\mathrm{M}-}=-\frac{1}{4}\left[-\bar{\psi} \psi+\psi^{\mathrm{T}} C \psi+\bar{\psi} C \bar{\psi}^{\mathrm{T}}-\psi^{\mathrm{T}} C C \bar{\psi}^{\mathrm{T}}\right]=\frac{1}{4}\left[2 \bar{\psi} \psi-\psi^{\mathrm{T}} C \psi-\bar{\psi} C \bar{\psi}^{\mathrm{T}}\right]
\end{aligned}
$$

since $C C=-1$ and $\bar{\psi} \psi=(\bar{\psi} \psi)^{\mathrm{T}}=-\psi^{\mathrm{T}} \bar{\psi}^{\mathrm{T}}$. Then

$$
\bar{\psi} \psi=\bar{\psi}_{\mathrm{M}+} \psi_{\mathrm{M}+}+\bar{\psi}_{\mathrm{M}-} \psi_{\mathrm{M}-} \cdot
$$




\section{SU(2) WITH 1 DIRAC (2 MAJORANA) FLAVOURS}

Similarly for the kinetic term,

$$
\begin{aligned}
& \bar{\psi}_{\mathrm{M}+} \not \partial \psi_{\mathrm{M}+}=\frac{1}{4}\left[\bar{\psi} \not \partial \psi+\psi^{\mathrm{T}} C \not \partial \psi+\bar{\psi} \not \partial C \bar{\psi}^{\mathrm{T}}+\psi^{\mathrm{T}} C \not \partial C \bar{\psi}^{\mathrm{T}}\right] \\
& \bar{\psi}_{\mathrm{M}-} \not \partial \psi_{\mathrm{M}-}=\frac{1}{4}\left[\bar{\psi} \not \partial \psi-\psi^{\mathrm{T}} C \not \partial \psi-\bar{\psi} \not \partial C \bar{\psi}^{\mathrm{T}}+\psi^{\mathrm{T}} C \not \partial C \bar{\psi}^{\mathrm{T}}\right],
\end{aligned}
$$

and so

$$
\bar{\psi}_{\mathrm{M}+} \not \partial \psi_{\mathrm{M}+}+\bar{\psi}_{\mathrm{M}-} \not \partial \psi_{\mathrm{M}-}=\frac{1}{2}\left[\bar{\psi} \not \partial \psi+\psi^{\mathrm{T}} C \not \partial C \bar{\psi}^{\mathrm{T}}\right]=\bar{\psi} \not \partial \psi,
$$

since $\gamma^{0}, \gamma^{2}$ anticommute with $C$ (because $C=-i \gamma^{2} \gamma^{0}$ ), $\gamma^{1}, \gamma^{3}$ commute with $C$ (for the same reason), but $\gamma^{1}, \gamma^{3}$ gain a minus sign on taking the transpose, whilst $\gamma^{0}, \gamma^{2}$ do not; additionally, a partial integral has been taken to turn the $\overleftarrow{\not}$ into a $\vec{\not}$.

We now have

$$
S_{\mathrm{f}}=\bar{\psi} \not \partial \psi+m \bar{\psi} \psi=\bar{\psi}_{\mathrm{M}+} \not \partial \psi_{\mathrm{M}+}+\bar{\psi}_{\mathrm{M}-} \not \partial \psi_{\mathrm{M}-}+m\left(\bar{\psi}_{\mathrm{M}+} \psi_{\mathrm{M}+}+\bar{\psi}_{\mathrm{M}-} \psi_{\mathrm{M}-}\right) .
$$

While this appears to admit an $\mathrm{SU}(2)$ global chiral symmetry, which would not break with a fermion mass, this is in fact not the case, since rotating between the Majorana flavours is not permitted. The $\chi \mathrm{SB}$ is best understood in terms of the Weyl basis, where it becomes evident that there is indeed a global $\mathrm{SU}(2)$ chiral symmetry, but in the presence of a nonzero fermionic condensate it breaks to $\mathrm{SO}(2)$.

\subsubsection{Spectroscopy}

As discussed in chapter 3 , to do spectroscopy we need to form operators of the correct quantum numbers. For a meson consisting of two Majorana fermions, one $\psi_{\mathrm{M}+}$ and one $\psi_{\mathrm{M}_{-}}$, an appropriate operator would take the form

$$
O_{+-}(x)=\bar{\psi}_{\mathrm{M}+} \Gamma \psi_{\mathrm{M}-},
$$

where $\Gamma$ is a combination of $\gamma_{i}$ s giving the requisite quantum numbers. We may also consider a meson consisting of two of the same Majorana flavour, for example, $\psi_{\mathrm{M}+}$. An appropriate operator is then

$$
O_{++}(x)=\bar{\psi}_{\mathrm{M}+} \Gamma \psi_{\mathrm{M}+}
$$


To find the masses of these states, as described in chapter 3, we must take correlation functions of these operators. The correlator in question takes the form $\left\langle O^{\dagger}(x) O(0)\right\rangle$.

\subsubsection{Reexpressing spectral quantities}

As discussed in the introduction to this section, we now want to find a way of expressing these specral quantities in terms of their Dirac equivalents.

\subsubsection{1 $O_{+-}$}

$$
\begin{aligned}
O_{+-} & =\frac{1}{4 i}\left(\bar{\psi} \Gamma \psi+\psi^{\mathrm{T}} C \Gamma \psi-\bar{\psi} \Gamma C \bar{\psi}^{\mathrm{T}}-\psi^{\mathrm{T}} C \Gamma C \bar{\psi}^{\mathrm{T}}\right) \\
& =\frac{1}{4 i}((1)+(2)+(3)+(4))
\end{aligned}
$$

We would like to reduce this to be in a more familiar form; to do so, we can find a few useful identities. Looking first at term (2), we notice that since it is a number, we expect it to equal its transpose. Applying this,

$$
\psi^{\mathrm{T}} C \Gamma \psi=-\psi^{\mathrm{T}}(C \Gamma)^{\mathrm{T}} \psi
$$

(where the - sign on the right-hand side has come from swapping the order of the spinors in the transpose to make the comparison clear). Comparing the two expressions, we see that either $C \Gamma=-(C \Gamma)^{\mathrm{T}}$, or $(2)=0$. Thus we may "switch off" the term if testing the first relation shows it is false. The same applies to term (3), with the same condition.

Meanwhile for term (4), using the same idea as above that the transpose of the term should equal the term itself, we see

$$
-\psi^{\mathrm{T}} C \Gamma C \bar{\psi}^{\mathrm{T}}=\bar{\psi} C \Gamma^{\mathrm{T}} C \psi= \pm \bar{\psi} \Gamma \psi
$$

(since $C$ is self-transpose) where the sign of the \pm is the same as that in the relation $C \Gamma^{\mathrm{T}} C= \pm \Gamma$. This relation will always hold, since $\Gamma$ is a combination of $\gamma_{i}$, which each pick up at most a change of sign on transposing, and $C$ (also being composed of $\gamma_{i} \mathrm{~s}$ ) 


\section{SU(2) WITH 1 DIRAC (2 MAJORANA) FLAVOURS}

will commute or anticommute with $\Gamma$. Thus $(4)= \pm(1)$; i.e.

$$
(1)+(4)= \begin{cases}0 & \text { if }(4)=-(1), \\ 2 \times(1) & \text { if }(4)=(1) .\end{cases}
$$

Thus by testing two relations, the expression may be cut down by at least one (potentially three) terms. In fact a minimum of two terms are culled, with either terms $(2)+(3)$ or $(1)+(4)$ dropping out, as is shown in table 4.1 .

\begin{tabular}{|c|c|c|c|c|}
\hline$\Gamma$ & $(C \Gamma)^{\mathrm{T}}$ & $\Rightarrow(2)+(3)$ & $\left(C \Gamma^{\mathrm{T}} C\right)$ & $\Rightarrow(1)+(4)$ \\
\hline \hline $\mathbb{1}$ & $-(C \Gamma)$ & $\checkmark$ & $-\Gamma$ & \\
\hline$\gamma_{0}$ & $+(C \Gamma)$ & & $+\Gamma$ & $\checkmark$ \\
\hline$\gamma$ & $+(C \Gamma)$ & & $+\Gamma$ & $\checkmark$ \\
\hline$\gamma_{0} \gamma$ & $+(C \Gamma)$ & & $+\Gamma$ & $\checkmark$ \\
\hline$\gamma_{5} \gamma_{0}$ & $-(C \Gamma)$ & $\checkmark$ & $-\Gamma$ & \\
\hline$\gamma_{5} \gamma$ & $-(C \Gamma)$ & $\checkmark$ & $-\Gamma$ & \\
\hline$\gamma_{0} \gamma_{5} \gamma$ & $+(C \Gamma)$ & & $+\Gamma$ & $\checkmark$ \\
\hline$\gamma_{5}$ & $-(C \Gamma)$ & $\checkmark$ & $-\Gamma$ & \\
\hline
\end{tabular}

Table 4.1: The terms in equation (4.24) that do not cancel are marked with a $\checkmark$.

This can be expressed more succinctly as:

$$
\bar{\psi}_{\mathrm{M}+} \Gamma \psi_{\mathrm{M}-}= \begin{cases}\frac{1}{4 i}\left(\psi^{\mathrm{T}} C \Gamma \psi-\bar{\psi} \Gamma C \bar{\psi}^{\mathrm{T}}\right) & \Gamma=\mathbb{1}, \gamma_{5} \gamma_{\mu}, \gamma_{5} \\ \frac{1}{2 i} \bar{\psi} \Gamma \psi & \Gamma=\gamma_{\mu}, \gamma_{0} \gamma, \gamma_{0} \gamma_{5} \gamma\end{cases}
$$

With the simplest forms of the meson operators in hand, we now look to calculate correlation functions of them. Let us take a detour via Dirac fermions: in the case of two Dirac flavours, the operator takes the form $O(x)=\bar{\psi}_{1} \Gamma \psi_{2}$, and the correlator:

$$
\begin{aligned}
\left\langle\left(\bar{\psi}_{1} \Gamma \psi_{2}\right)^{\dagger}(x)\left(\bar{\psi}_{1} \Gamma \psi_{2}\right)(0)\right\rangle & =\left\langle\psi_{2}^{\dagger}(x) \Gamma^{\dagger} \gamma_{0} \psi_{1}(x) \bar{\psi}_{1}(0) \Gamma \psi_{2}(0)\right\rangle \\
& =\left\langle\bar{\psi}_{2}(x) \gamma_{0} \Gamma^{\dagger} \gamma_{0} \psi_{1}(x) \bar{\psi}_{1}(0) \Gamma \psi_{2}(0)\right\rangle \\
& =\left\langle\bar{\psi}_{2}(x) \bar{\Gamma} \psi_{1}(x) \bar{\psi}_{1}(0) \Gamma \psi_{2}(0)\right\rangle \quad\left(\bar{\Gamma}=\gamma_{0} \Gamma^{\dagger} \gamma_{0}\right)
\end{aligned}
$$

with $\bar{\psi}_{a} \psi_{b}$ indicating a Wick contraction. We have defined $\bar{\Gamma}=\gamma_{0} \Gamma^{\dagger} \gamma_{0}$, and only one possible set of contractions is available, since $\bar{\psi}_{1} \psi_{2}$ is not permitted. Rearranging and 
performing the contraction,

$$
\begin{aligned}
\left\langle\left(\bar{\psi}_{1} \Gamma \psi_{2}\right)^{\dagger}(x)\left(\bar{\psi}_{1} \Gamma \psi_{2}\right)(0)\right\rangle & =-\left\langle\bar{\Gamma} \vec{\psi}_{1}(x) \bar{\psi}_{1}(0) \Gamma \stackrel{\psi_{2}(0) \bar{\psi}_{2}}{ }(x)\right\rangle \\
& =-\operatorname{tr} \bar{\Gamma} D^{-1}(x ; 0) \Gamma D^{-1}(0 ; x)
\end{aligned}
$$

Now if we turn our attention back to the two-Majorana case, we see that one of the cases appears to have a similar form, $O(x)=\frac{1}{2 i} \bar{\psi} \Gamma \psi$. However, since there is only one Dirac flavour present, an additional set of contractions is available:

$$
\begin{aligned}
\left\langle(\bar{\psi} \Gamma \psi)^{\dagger}(x)(\bar{\psi} \Gamma \psi)(0)\right\rangle & =\left\langle\psi^{\dagger}(x) \Gamma^{\dagger} \gamma_{0} \psi(x) \bar{\psi}(0) \Gamma \psi(0)\right\rangle \\
& =\left\langle\bar{\psi}(x) \gamma_{0} \Gamma^{\dagger} \gamma_{0} \psi(x) \bar{\psi}(0) \Gamma \psi(0)\right\rangle \\
& =\langle\bar{\psi}(x) \bar{\Gamma} \bar{\psi}(x) \bar{\psi}(0) \Gamma \psi(0)\rangle \\
& =\frac{1}{4}(-\langle\bar{\Gamma} \psi(x) \bar{\psi}(0) \Gamma \bar{\psi}(0) \bar{\psi}(x)\rangle+\langle\bar{\Gamma} \overrightarrow{\psi(x) \bar{\psi}}(x) \Gamma \bar{\psi}(0) \bar{\psi}(0)\rangle) \\
& =\frac{1}{4}\left(\operatorname{tr} \bar{\Gamma} D^{-1}(x ; x) \Gamma D^{-1}(0 ; 0)-\operatorname{tr} \bar{\Gamma} D^{-1}(x ; 0) \Gamma D^{-1}(0 ; x)\right)
\end{aligned}
$$

The second term here looks like the familiar one above, and lends itself to analysis using Z2SEMWall sources as described in section 3.2.2:, but the first term is more problematic. The propagators represent disconnected diagrams, and they entail cancellations which result in any signal being swamped by statistical noise. Since the techniques for dealing with these terms (discussed in section 3.2.2.2) are more demanding both computationally and analytically, the channels without such a term were considered first. Preliminary results from the study including the disconnected channels are included below.

Moving on to the other set of channels, the operator $O(x)=\frac{1}{4 i}\left(\psi^{\mathrm{T}} C \Gamma \psi-\bar{\psi} \Gamma C \bar{\psi}^{\mathrm{T}}\right)$ multiplies out to four terms; however two immediately drop out since the $\psi$ and $\bar{\psi}$ are unmatched; this leaves:

$$
\begin{aligned}
& \left\langle\left(\psi^{\mathrm{T}} C \Gamma \psi-\bar{\psi} \Gamma C \bar{\psi}^{\mathrm{T}}\right)^{\dagger}(x)\left(\psi^{\mathrm{T}} C \Gamma \psi-\bar{\psi} \Gamma C \bar{\psi}^{\mathrm{T}}\right)(0)\right\rangle \\
& =\left\langle\left(\psi^{\mathrm{T}} C \Gamma \psi\right)^{\dagger}(x)\left(\psi^{\mathrm{T}} C \Gamma \psi\right)(0)\right\rangle+\left\langle\left(\bar{\psi} \Gamma C \bar{\psi}^{\mathrm{T}}\right)^{\dagger}(x)\left(\bar{\psi} \Gamma C \bar{\psi}^{\mathrm{T}}\right)(0)\right\rangle .
\end{aligned}
$$


Evaluating these two terms in turn; for the first term:

$$
\begin{aligned}
& \left\langle\left(\psi^{\mathrm{T}} C \Gamma \psi\right)^{\dagger}(x)\left(\psi^{\mathrm{T}} C \Gamma \psi\right)(0)\right\rangle \\
& =\left\langle\psi^{\dagger}(x) \Gamma^{\dagger} C \psi^{*}(x) \psi^{\mathrm{T}}(0) C \Gamma \psi(0)\right\rangle \\
& =\left\langle\bar{\psi}(x) \gamma_{0} \Gamma^{\dagger} C \gamma_{0} \bar{\psi}^{\mathrm{T}}(x) \psi^{\mathrm{T}}(0) C \Gamma \psi(0)\right\rangle \\
& =-\left\langle\bar{\psi}(x) \bar{\Gamma} C \bar{\psi}^{\mathrm{T}}(x) \psi^{\mathrm{T}}(0) C \Gamma \psi(0)\right\rangle \\
& =-\frac{1}{Z} \int \mathcal{D} \bar{\psi} \mathcal{D} \psi \bar{\psi}_{a A}(x) \bar{\Gamma}_{a b} C_{b c} \bar{\psi}_{c B}^{\mathrm{T}}(x) \psi_{d A}^{\mathrm{T}}(0) C_{d e} \Gamma_{e f} \psi_{f b}(0) e^{-S} \\
& =-\frac{1}{Z} \int \mathcal{D} \bar{\psi} \mathcal{D} \psi\left[\operatorname{tr} \bar{\Gamma}_{a b} C_{b c} \psi_{d A}^{\mathrm{T}}(0) \bar{\psi}_{c B}^{\mathrm{T}}(x) C_{d e} \Gamma_{e f} \psi_{f B}(0) \bar{\psi}_{a A}(x)\right. \\
& \left.\quad-\operatorname{tr} \bar{\Gamma}_{a b} C_{b c} \psi_{d A}^{\mathrm{T}}(0) \bar{\psi}_{a A}(x) C_{d e} \Gamma_{e f} \psi_{f B}(0) \bar{\psi}_{c B}^{\mathrm{T}}(x)\right] e^{-S} \\
& =-\left[\operatorname{tr} \bar{\Gamma}_{a b} C_{b c} D_{d B, c A}^{-1}(0 ; x) C_{d e} \Gamma_{e f} D_{f A, a B}^{-1}(0 ; x)\right. \\
& \left.\quad-\operatorname{tr} \bar{\Gamma}_{a b} C_{b c} D_{d A, a A}^{-1}(0 ; x) C_{d e} \Gamma_{e f} D_{f B, c B}(0 ; x)\right] \\
& =-\operatorname{tr} \bar{\Gamma} C D_{A B}^{-1 \mathrm{~T}}(0 ; x) C \Gamma D_{B A}^{-1}(0 ; x)+\operatorname{tr}(\bar{\Gamma} C)^{\mathrm{T}} D_{A A}^{-1 \mathrm{~T}}(0 ; x) C \Gamma D_{B B}^{-1}(0 ; x),
\end{aligned}
$$

and since $(\bar{\Gamma} C)^{\mathrm{T}}=-\bar{\Gamma} C$ follows from the condition defining where this case is used,

$$
\left\langle\left(\psi^{\mathrm{T}} C \Gamma \psi\right)^{\dagger}(x)\left(\psi^{\mathrm{T}} C \Gamma \psi\right)(0)\right\rangle=-2 \operatorname{tr} \bar{\Gamma} C D^{-1 \mathrm{~T}}(0 ; x) C \Gamma D^{-1}(0 ; x)
$$

For the second term:

$$
\begin{aligned}
& \left\langle\left(\bar{\psi} \Gamma C \bar{\psi}^{\mathrm{T}}\right)^{\dagger}(x)\left(\bar{\psi} \Gamma C \bar{\psi}^{\mathrm{T}}\right)(0)\right\rangle \\
& =\left\langle\bar{\psi}^{*}(x) C \Gamma^{\dagger} \bar{\psi}^{\dagger}(x) \bar{\psi}(0) \Gamma C \bar{\psi}^{\mathrm{T}}(0)\right\rangle \\
& =\left\langle\psi^{\mathrm{T}}(x) \gamma_{0} C \Gamma^{\dagger} \gamma_{0} \psi(x) \bar{\psi}(0) \Gamma C \bar{\psi}^{\mathrm{T}}(0)\right\rangle \\
& =-\left\langle\bar{\psi}^{\mathrm{T}}(x) C \bar{\Gamma} \bar{\psi}(x) \bar{\psi}(0) \Gamma C \bar{\psi}^{\mathrm{T}}(0)\right\rangle \\
& =-\frac{1}{Z} \int D \bar{\psi} D \psi \psi_{a A} C_{a b} \bar{\Gamma}_{b c} \psi_{c A}(x) \bar{\psi}_{d B} \Gamma_{d e} C_{e f} \bar{\psi}_{f B}(0) e^{-S}
\end{aligned}
$$




$$
\begin{aligned}
=-\frac{1}{Z} \int D \bar{\psi} D \psi\left[C_{a b} \bar{\Gamma}_{b c} \psi_{c A}(x) \bar{\psi}_{d B}(0) \Gamma_{d e} C_{e f} \psi_{a A}(x) \bar{\psi}_{f B}(0)\right. \\
\left.\quad-C_{a b} \bar{\Gamma}_{b c} \psi_{a A}(x) \bar{\psi}_{d B}(0) \Gamma_{d e} C_{e f} \psi_{c A}(x) \bar{\psi}_{f B}(0)\right] e^{-S} \\
=-\left[\operatorname{tr} C_{a b} \bar{\Gamma}_{b c} D_{c A, d B}^{-1}(x ; 0) \Gamma_{d e} C_{e f} D_{a A, f B}^{-1}(x ; 0)\right. \\
\left.\quad \quad \operatorname{tr} C_{a b} \bar{\Gamma}_{b c} D_{a A, d B}^{-1}(x ; 0) \Gamma_{d e} C_{e f} D_{c A, f B}^{-1}(x ; 0)\right] \\
=-\operatorname{tr} C \bar{\Gamma} D^{-1}(x ; 0) \Gamma C D^{-1 \mathrm{~T}}(x ; 0)+\operatorname{tr}(C \bar{\Gamma})^{\mathrm{T}} D^{-1}(x ; 0) \Gamma C D^{-1 \mathrm{~T}}(x ; 0) \\
=-2 \operatorname{tr} C \bar{\Gamma} D^{-1}(x ; 0) \Gamma C D^{-1 \mathrm{~T}}(x ; 0) \\
=-2 \operatorname{tr} C \bar{\Gamma} \gamma_{5} D^{-1 \dagger}(0 ; x) \gamma_{5} \Gamma C \gamma_{5} D^{-1 *}(0 ; x) \gamma_{5} \\
=-2 \operatorname{tr} D^{-1 \dagger}(0 ; x) \Gamma C D^{-1 *}(0 ; x) C \bar{\Gamma} \\
=-2 \operatorname{tr}\left(\bar{\Gamma}^{\dagger} C D^{-1 \mathrm{~T}}(0 ; x) C \Gamma^{\dagger} D^{-1}(0 ; x)\right)^{\dagger} \\
=-2 \operatorname{tr}\left(\bar{\Gamma} C D^{-1 \mathrm{~T}}(0 ; x) C \Gamma D^{-1}(0 ; x)\right)^{\dagger},
\end{aligned}
$$

where we have used:

- the cyclicity of the trace

- $\gamma_{5} \Gamma \gamma_{5}= \pm \Gamma \Leftrightarrow \gamma_{5} \bar{\Gamma} \gamma_{5}= \pm \bar{\Gamma}$

- $\Gamma^{\dagger}= \pm \Gamma \Leftrightarrow \bar{\Gamma}^{\dagger}= \pm \bar{\Gamma}$

- $\gamma_{5} D^{-1 \dagger}(y ; x) \gamma_{5}=D^{-1}(x ; y)$

Summing these contributions, the meson correlator for $O(x)=\bar{\psi}_{\mathrm{M}+}(x) \Gamma \psi_{\mathrm{M}_{-}}(x)$, $\Gamma=\mathbb{1}, \gamma_{5} \gamma_{\mu}, \gamma_{5}$ is

$$
\left\langle O^{\dagger}(x) O(0)\right\rangle=-\frac{1}{8}\left[F(0 ; x)+F^{\dagger}(0 ; x)\right]
$$

where

$$
F(0 ; x)=\operatorname{tr} \bar{\Gamma} C D^{-1 \mathrm{~T}}(0 ; x) C \Gamma D^{-1}(0 ; x)
$$

We may permute the $C$ s out to give

$$
F(0 ; x)=\operatorname{tr} \bar{\Gamma} D^{-1}(x ; 0) \Gamma D^{-1}(0 ; x)
$$

which is the same form as the two-flavour Dirac correlator of equation (4.34). Taking 


\section{SU(2) WITH 1 DIRAC (2 MAJORANA) FLAVOURS}

the Hermitian conjugate of this,

$$
\begin{aligned}
F^{\dagger}(0 ; x) & =\operatorname{tr} D^{-1 \dagger}(0 ; x) \Gamma^{\dagger} D^{-1 \dagger}(x ; 0) \bar{\Gamma}^{\dagger} \\
& =\operatorname{tr} \bar{\Gamma} D^{-1 \mathrm{~T}}(0 ; x) \Gamma D^{-1 \mathrm{~T}}(x ; 0) \\
& =\operatorname{tr} C \bar{\Gamma} C D^{-1}(x ; 0) C \Gamma C D^{-1}(0 ; x) \\
& =\operatorname{tr} \bar{\Gamma} D^{-1}(x ; 0) \Gamma D^{-1}(0 ; x) ;
\end{aligned}
$$

i.e. we have ended back up at the same form as $F(0 ; x)$. Thus,

$$
\left\langle O_{+-}^{\dagger}(x) O_{+-}(0)\right\rangle=-\frac{1}{4} \operatorname{tr} \bar{\Gamma} D^{-1}(x ; 0) \Gamma D^{-1}(0 ; x) .
$$

We now have expressions for $O_{+-}^{\dagger} O_{+-}$for all possible $\Gamma$ s:

$$
\left\langle O_{+-}^{\dagger}(x) O_{+-}(0)\right\rangle=\left\{\begin{aligned}
-\frac{1}{4} \operatorname{tr} \bar{\Gamma} D^{-1}(x ; 0) \Gamma D^{-1}(0 ; x) & \Gamma=\mathbb{1}, \gamma_{5} \gamma_{\mu}, \gamma_{5} \\
\frac{1}{4}\left(\operatorname{tr} \bar{\Gamma} D^{-1}(x ; x) \Gamma D^{-1}(0 ; 0)\right. & \\
\left.-\operatorname{tr} \bar{\Gamma} D^{-1}(x ; 0) \Gamma D^{-1}(0 ; x)\right) & \Gamma=\gamma_{\mu}, \gamma_{0} \gamma, \gamma_{0} \gamma_{5} \gamma
\end{aligned}\right.
$$

\subsubsection{PCAC}

Recall from chapter 3 that the Wilson fermion formulation requires tuning of the bare fermion mass to restore chiral symmetry, which was explicitly broken. To do this we make use of the axial anomaly, or partially conserved axial current (PCAC). This is fully conserved in the presence of chiral symmetry, so we look for its derivative to be zero to indicate the correct bare mass. The PCAC mass is defined as

$$
m_{\mathrm{PCAC}}=\frac{\sum_{\mathbf{x}}\left\langle\partial_{0} A_{0}(x) P(0)\right\rangle}{2 \sum_{\mathbf{x}}\langle P(x) P(0)\rangle},
$$

where

$$
\begin{aligned}
A_{0}(x) & =\bar{\psi}_{\mathrm{M}+}(x) \gamma_{0} \gamma_{5} \psi_{\mathrm{M}-}(x) \\
P(x) & =\bar{\psi}_{\mathrm{M}+}(x) \gamma_{5} \psi_{\mathrm{M}-}(x)
\end{aligned}
$$

The denominator is simply the meson correlator above with $\Gamma=\gamma_{5}$; the numerator meanwhile is in effect a "mixed" correlator and requires special attention. Going 
through the same procedure as above shows:

$$
\left\langle\left(\bar{\psi}_{\mathrm{M}+} \gamma_{5} \psi_{\mathrm{M}-}\right)^{\dagger}(x)\left(\bar{\psi}_{\mathrm{M}+} \gamma_{0} \gamma_{5} \psi_{\mathrm{M}-}\right)(0)\right\rangle=-\frac{1}{4} \operatorname{tr} \overline{\gamma_{5}} D^{-1}(x ; 0) \gamma_{0} \gamma_{5} D^{-1}(0 ; x) .
$$

Happily, all of the results found thus far equate (up to multiplicative constants, which drop out when ratios are taken) to 1-flavour Dirac correlators; thus both code for and results from simulations of 1 Dirac flavour also apply to and give results for 2 Majorana flavours.

\subsubsection{3 $O_{++}$}

This case has not been looked at in detail for this project; however, if one were interested, the cancellations would work differently. The operator would take the form

$$
\bar{\psi}_{\mathrm{M}+} \Gamma \psi_{\mathrm{M}+}= \begin{cases}\frac{1}{4}\left(2 \bar{\psi} \Gamma \psi+\psi^{\mathrm{T}} C \Gamma \psi+\bar{\psi} \Gamma C \bar{\psi}^{\mathrm{T}}\right) & \Gamma=\mathbb{1}, \gamma_{5} \gamma_{\mu}, \gamma_{5} \\ 0 & \Gamma=\gamma_{\mu}, \gamma_{0} \gamma, \gamma_{0} \gamma_{5} \gamma\end{cases}
$$

and then the correlator

$$
\left\langle O_{++}^{\dagger}(x) O_{++}(0)\right\rangle=\left\{\begin{aligned}
\frac{1}{4}\left[\operatorname{tr}\left(\bar{\Gamma} D^{-1}(x ; x)\right) \operatorname{tr}\left(\Gamma D^{-1}(0 ; 0)\right)\right. & \\
\left.-2 \operatorname{tr}\left(\bar{\Gamma} D^{-1}(x ; 0) \Gamma D^{-1}(0 ; x)\right)\right] & \Gamma=\mathbb{1}, \gamma_{5} \gamma_{\mu}, \gamma_{5} \\
0 & \Gamma=\gamma_{\mu}, \gamma_{0} \gamma, \gamma_{0} \gamma_{5} \gamma
\end{aligned}\right.
$$

Although the overall form is different to the one Dirac flavour case, the two terms are both of the same form as their Dirac counterparts, thus they would be accessible from the same code and output. 


\begin{tabular}{|c|c|}
\hline 1 & \\
\hline $\mathbb{1}, \gamma_{5} \gamma_{\mu}, \gamma_{5}$ & $\begin{aligned} O_{+-} & =\frac{1}{4 i}\left(\psi^{\mathrm{T}} C \Gamma \psi-\bar{\psi} \Gamma C \bar{\psi}^{\mathrm{T}}\right) \\
\left\langle O_{+-}^{\dagger}(x) O_{+-}(0)\right\rangle= & -\frac{1}{4} \operatorname{tr} \bar{\Gamma} D^{-1}(x ; 0) \Gamma D^{-1}(0 ; x) \\
O_{++}= & \frac{1}{4}\left(2 \bar{\psi} \Gamma \psi+\psi^{\mathrm{T}} C \Gamma \psi+\bar{\psi} \Gamma C \bar{\psi}^{\mathrm{T}}\right) \\
\left\langle O_{++}^{\dagger}(x) O_{++}(0)\right\rangle= & \frac{1}{4}\left[\operatorname{tr}\left(\bar{\Gamma} D^{-1}(x ; x)\right) \operatorname{tr}\left(\Gamma D^{-1}(0 ; 0)\right)\right. \\
& \left.-2 \operatorname{tr}\left(\bar{\Gamma} D^{-1}(x ; 0) \Gamma D^{-1}(0 ; x)\right)\right]\end{aligned}$ \\
\hline$\gamma_{\mu}, \gamma_{0} \gamma, \gamma_{0} \gamma_{5} \gamma$ & $\begin{aligned} O_{+-} & =\frac{1}{2 i} \bar{\psi} \Gamma \psi \\
\left\langle O_{+-}^{\dagger}(x) O_{+-}(0)\right\rangle= & \frac{1}{4}\left(\operatorname{tr} \bar{\Gamma} D^{-1}(x ; x) \Gamma D^{-1}(0 ; 0)\right. \\
& \left.-\operatorname{tr} \bar{\Gamma} D^{-1}(x ; 0) \Gamma D^{-1}(0 ; x)\right) \\
O_{++} & =\left\langle O_{++}^{\dagger}(x) O_{++}(0)\right\rangle=0\end{aligned}$ \\
\hline
\end{tabular}

Table 4.2: A summary of the important results of section 4.1.

\subsection{Results}

Since the theory has not been investigated on the lattice before, we are somewhat "shooting in the dark" with regards to the parameters to use. Based on related theories, we may make an educated guess as to where the region of interest will be, but a preliminary study is necessary to narrow down this range to make simulation of interesting physics feasible. If $\beta$ is taken too small, i.e. large $a$, then all that is observed is a theory of lattice artefacts (the "bulk"), since the lattice becomes too coarse to resolve physics. However, the larger $\beta$ is taken, the larger lattices must be to achieve the same physical size, which increases simulation time and expense rapidly. Meanwhile if $m$ is taken too small, then we in effect have a negative fermion mass, and so end up simulating numerical artefacts; if $m$ is very large, then the fermions become heavy and might as well be quenched, not to mention that we will be distant from the chiral region of interest.

To this end the pilot study sought to map the phase diagram, by looking at the average plaquette on a $4^{4}$ lattice; the plaquette is a cheap observable to calculate and is not severely afflicted by finite-size effects. We expect to see a phase transition due to the bulk effects, visible as a sharp jump in the average plaquette. We choose a value of $\beta$ lying above this, where the transition has turned into a crossover, but as 
close as possible without risking entering the bulk phase, to avoid having too small a lattice spacing. Once the bulk transition has been avoided, any remaining changes in the plaquette are likely to be due to a physical chiral transition.

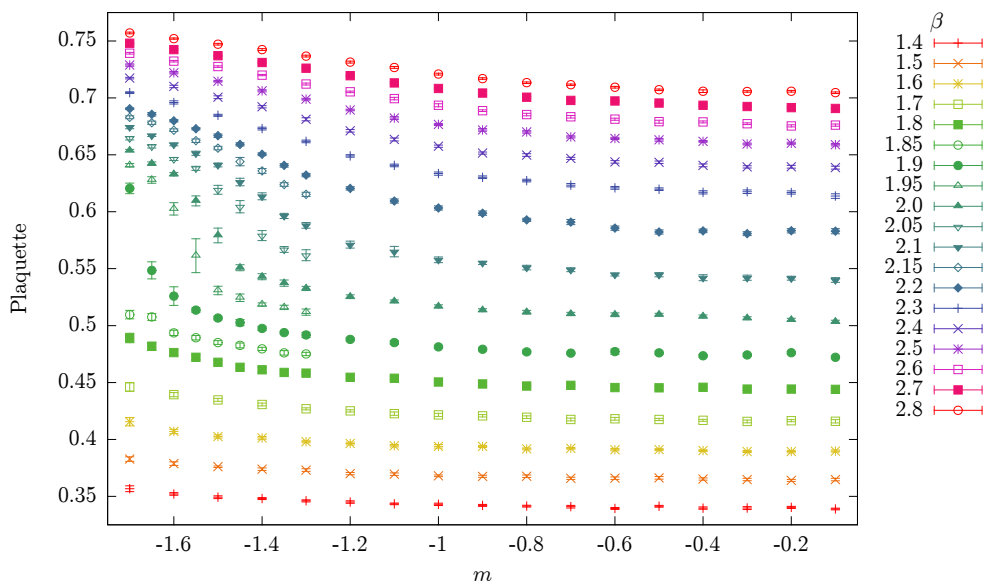

Figure 4.1: The phase diagram of the theory, showing the average plaquette across a range of value of $\beta$ and $m$; the region of interest was identified around $\beta=2.05, m=-1.5$.

Runs were performed between $1.4 \leq \beta \leq 2.8$ and $-1.7 \leq m \leq 0.1$, with a resolution of 0.1 in each direction; once the region of interest was narrowed down, additional runs were taken every 0.05 in each direction for a closer look. The results of this study are shown in figure 4.1; the bulk phase transition is visible, for example, at $\beta=1.9$, $m=-1.65$. The region of interest was found to lie at around $\beta=2.05, m=-1.5$.

\subsubsection{Monte Carlo}

To ensure that the results are on the firmest possible footing, we chose now to narrow our focus to a single $\beta$, namely $\beta=2.05$, with the intention of revisiting other values of $\beta$ once the theory is better-explored. At this $\beta$ value, simulations were performed at lattice sizes of $16 \times 8^{3}, 24 \times 12^{3}, 32 \times 16^{3}$, and $48 \times 24^{3},{ }^{1}$ at values of the bare fermion mass between $-1.523 \leq m \leq-1.475$, with $m=-1.523$ being the closest point obtainable to the chiral limit; going beyond this required larger lattices than could be reasonably simulated with available resources. The parameters used for each run are shown in table 4.3 .

\footnotetext{
${ }^{1}$ An exploratory run was perfomed at a lattice size of $64 \times 32^{3}$; with the results used only for the visualisations discussed in appendix A.
} 


\begin{tabular}{c|cc|cc|ccc|c}
\hline Lattice & $V$ & $-a m_{0}$ & $N_{\text {conf }}$ & Acceptance & $N_{\text {pf }}$ & $t_{\text {len }}$ & $n_{\text {steps }}$ & Machine \\
\hline A1 & $16 \times 8^{3}$ & 1.475 & 2400 & $91.4 \%$ & 1 & 1.0 & 10 & SC \\
A2 & $16 \times 8^{3}$ & 1.500 & 2200 & $90.9 \%$ & 1 & 1.0 & 10 & SC, UL \\
A3 & $16 \times 8^{3}$ & 1.510 & 2400 & $89.8 \%$ & 1 & 1.0 & 10 & SC, UL \\
A4 & $16 \times 8^{3}$ & 1.510 & 4000 & $92.4 \%$ & 2 & 1.0 & 8 & SC \\
\hline B1 & $24 \times 12^{3}$ & 1.475 & 2400 & $79.9 \%$ & 1 & 1.0 & 10 & SC, UL \\
B2 & $24 \times 12^{3}$ & 1.500 & 2300 & $78.7 \%$ & 1 & 1.0 & 10 & SC, UL \\
B3 & $24 \times 12^{3}$ & 1.510 & 4000 & $88.5 \%$ & 2 & 1.0 & 10 & SC, UL \\
\hline C1 & $32 \times 16^{3}$ & 1.475 & 2100 & $90.6 \%$ & 1 & 1.0 & 20 & SC \\
C2 & $32 \times 16^{3}$ & 1.490 & 2300 & $90.0 \%$ & 1 & 1.0 & 20 & SC, UL \\
C3 & $32 \times 16^{3}$ & 1.510 & 2200 & $89.4 \%$ & 1 & 1.0 & 20 & UL \\
C4 & $32 \times 16^{3}$ & 1.510 & 2300 & $83.2 \%$ & 2 & 4.0 & 45 & BGP \\
C5 & $32 \times 16^{3}$ & 1.514 & 2300 & $89.8 \%$ & 1 & 1.0 & 20 & UL, BGP \\
C6 & $32 \times 16^{3}$ & 1.519 & 2300 & $81.8 \%$ & 1 & 1.0 & 20 & UL, BGP \\
C7 & $32 \times 16^{3}$ & 1.523 & 2200 & $88.0 \%$ & 1 & 1.0 & 20 & SC \\
\hline D1 & $48 \times 24^{3}$ & 1.510 & 1534 & $80.5 \%$ & 2 & 4.0 & 65 & BGP \\
D2 & $48 \times 24^{3}$ & 1.523 & 2168 & $91.4 \%$ & 1 & 1.0 & 40 & BGP \\
\hline
\end{tabular}

Table 4.3: Lattices considered and parameters used. In the Machine column, SC refers to an x86-64 cluster in Swansea University, UL to the ULGQCD machine in Liverpool, and BGP to the 'pyxis' Blue Gene/P machine (part of the STFC DiRAC facility) in Swansea University.

\begin{tabular}{ccc|c|cc}
\hline Lattice & $V$ & $-a m_{0}$ & Block size & $a m_{\mathrm{PCAC}}$ & $a m_{\gamma_{5}}$ \\
\hline $\mathrm{A} 1$ & $16 \times 8^{3}$ & 1.475 & 30 & $0.1489(9)$ & $0.9718(31)$ \\
$\mathrm{A} 2$ & $16 \times 8^{3}$ & 1.500 & 30 & $0.1101(12)$ & $0.8408(47)$ \\
$\mathrm{A} 3$ & $16 \times 8^{3}$ & 1.510 & 30 & $0.0904(14)$ & $0.7618(64)$ \\
$\mathrm{A} 4$ & $16 \times 8^{3}$ & 1.510 & 30 & $0.0872(22)$ & $0.747(10)$ \\
\hline $\mathrm{B} 1$ & $24 \times 12^{3}$ & 1.475 & 30 & $0.1493(5)$ & $0.9728(18)$ \\
$\mathrm{B} 2$ & $24 \times 12^{3}$ & 1.500 & 30 & $0.1113(8)$ & $0.8449(31)$ \\
$\mathrm{B} 3$ & $24 \times 12^{3}$ & 1.510 & 40 & $0.09226(92)$ & $0.7694(40)$ \\
\hline $\mathrm{C} 1$ & $32 \times 16^{3}$ & 1.475 & 30 & $0.1485(4)$ & $0.9700(14)$ \\
$\mathrm{C} 2$ & $32 \times 16^{3}$ & 1.490 & 30 & $0.1279(2)$ & $0.9039(9)$ \\
$\mathrm{C} 3$ & $32 \times 16^{3}$ & 1.510 & 30 & $0.09111(31)$ & $0.7646(15)$ \\
$\mathrm{C} 4$ & $32 \times 16^{3}$ & 1.510 & 40 & $0.09048(52)$ & $0.7616(25)$ \\
$\mathrm{C} 5$ & $32 \times 16^{3}$ & 1.514 & 30 & $0.08223(34)$ & $0.7256(18)$ \\
$\mathrm{C} 6$ & $32 \times 16^{3}$ & 1.519 & 30 & $0.06587(37)$ & $0.6468(22)$ \\
$\mathrm{C} 7$ & $32 \times 16^{3}$ & 1.523 & 30 & $0.04840(54)$ & $0.5480(36)$ \\
\hline D1 & $48 \times 24^{3}$ & 1.510 & 40 & $0.09130(27)$ & $0.7653(14)$ \\
$\mathrm{D} 2$ & $48 \times 24^{3}$ & 1.523 & 30 & $0.04722(43)$ & $0.5412(29)$ \\
\hline
\end{tabular}

Table 4.4: PCAC and pseudoscalar meson mass. 


\begin{tabular}{ccc|c|cc}
\hline Lattice & $V$ & $-a m_{0}$ & Block size & $a \sqrt{\sigma}$ & $a m_{0^{++}}$ \\
\hline A1 & $16 \times 8^{3}$ & 1.475 & - & $0.424(13)$ & $0.8422 \pm 0.0968$ \\
A2 & $16 \times 8^{3}$ & 1.500 & - & $0.335(10)$ & $0.7320 \pm 0.0885$ \\
A3 & $16 \times 8^{3}$ & 1.510 & - & $0.299(12)$ & $0.5690 \pm 0.0585$ \\
A4 & $16 \times 8^{3}$ & 1.510 & 160 & - & $0.5873 \pm 0.0553$ \\
\hline B1 & $24 \times 12^{3}$ & 1.475 & - & $0.378(19)$ & $0.9582 \pm 0.1174$ \\
B2 & $24 \times 12^{3}$ & 1.500 & 60 & - & $0.7296 \pm 0.1092$ \\
B3 & $24 \times 12^{3}$ & 1.510 & 191 & $0.322(10)$ & $0.5284 \pm 0.1494$ \\
\hline C1 & $32 \times 16^{3}$ & 1.475 & - & $0.436(60)$ & $0.9654 \pm 0.1057$ \\
C2 & $32 \times 16^{3}$ & 1.490 & - & $0.379(44)$ & $0.8265 \pm 0.0644$ \\
C3 & $32 \times 16^{3}$ & 1.510 & - & $0.318(11)$ & $0.5985 \pm 0.0573$ \\
C4 & $32 \times 16^{3}$ & 1.510 & 103 & - & $0.5901 \pm 0.0438$ \\
C5 & $32 \times 16^{3}$ & 1.514 & - & $0.322(13)$ & $0.5530 \pm 0.0415$ \\
C6 & $32 \times 16^{3}$ & 1.519 & - & $0.2859(75)$ & $0.3689 \pm 0.0437$ \\
C7 & $32 \times 16^{3}$ & 1.523 & - & $0.2368(84)$ & $0.3146 \pm 0.0278$ \\
\hline D1 & $48 \times 24^{3}$ & 1.510 & 59 & - & $0.4609 \pm 0.0553$ \\
D2 & $48 \times 24^{3}$ & 1.523 & - & $0.2354(56)$ & $0.3595 \pm 0.0219$ \\
\hline
\end{tabular}

Table 4.5: Glueball masses and string tension, as computed by Biagio Lucini and Andreas Athenodorou.

\begin{tabular}{c|cc|cccc}
\hline Lattice & $V$ & $-a m_{0}$ & $m_{\frac{1}{2}}$ & $m_{\mathbb{1}}$ & $m_{\gamma_{\mu}}$ & $m_{\gamma_{5} \gamma_{\mu}}$ \\
\hline B1 & $24 \times 12^{3}$ & 1.475 & $1.707(20)$ & - & $1.1602(11)$ & $2.303(50)$ \\
B2 & $24 \times 12^{3}$ & 1.500 & $1.579(54)$ & $0.64(20)$ & $1.0504(18)$ & $2.063(40)$ \\
B3 & $24 \times 12^{3}$ & 1.510 & $1.3524(81)$ & $0.48(20)$ & - & - \\
\hline C4 & $32 \times 16^{3}$ & 1.510 & $1.334(18)$ & $0.65(21)$ & - & - \\
C5 & $32 \times 16^{3}$ & 1.514 & $1.242(55)$ & $0.403(80)$ & $0.9426(28)$ & $1.678(73)$ \\
C6 & $32 \times 16^{3}$ & 1.519 & $1.136(45)$ & $0.296(96)$ & $0.8639(41)$ & $1.683(62)$ \\
\hline D2 & $48 \times 24^{3}$ & 1.523 & $0.900(41)$ & $0.28(12)$ & $0.759(24)$ & $1.21(51)$ \\
\hline
\end{tabular}

Table 4.6: Masses of the spin- $\frac{1}{2}$ particle and of the scalar, vector, and axial vector mesons (including the disconnected contribution where appropriate), as computed by Georg Bergner.

\begin{tabular}{c|cc|cc}
\hline Lattice & $V$ & $-a m_{0}$ & $a$ & $b$ \\
\hline A4 & $16 \times 8^{3}$ & 1.510 & $0.06535(6)$ & $-0.08876(7)$ \\
\hline B3 & $24 \times 12^{3}$ & 1.510 & $0.45572(2)$ & $-0.25534(3)$ \\
\hline C4 & $32 \times 16^{3}$ & 1.510 & $0.07630(4)$ & $-0.08542(5)$ \\
\hline D1 & $48 \times 24^{3}$ & 1.510 & $0.02322(2)$ & $-0.05136(2)$ \\
D2 & $48 \times 24^{3}$ & 1.523 & $0.17588(2)$ & $-0.14952(2)$ \\
\hline
\end{tabular}

Table 4.7: Results of the fit of the static potential from Wilson loops as $V(r)=a+b / r+\sigma r$, with $\sigma$ set to the value found from Polyakov loop correlators. 


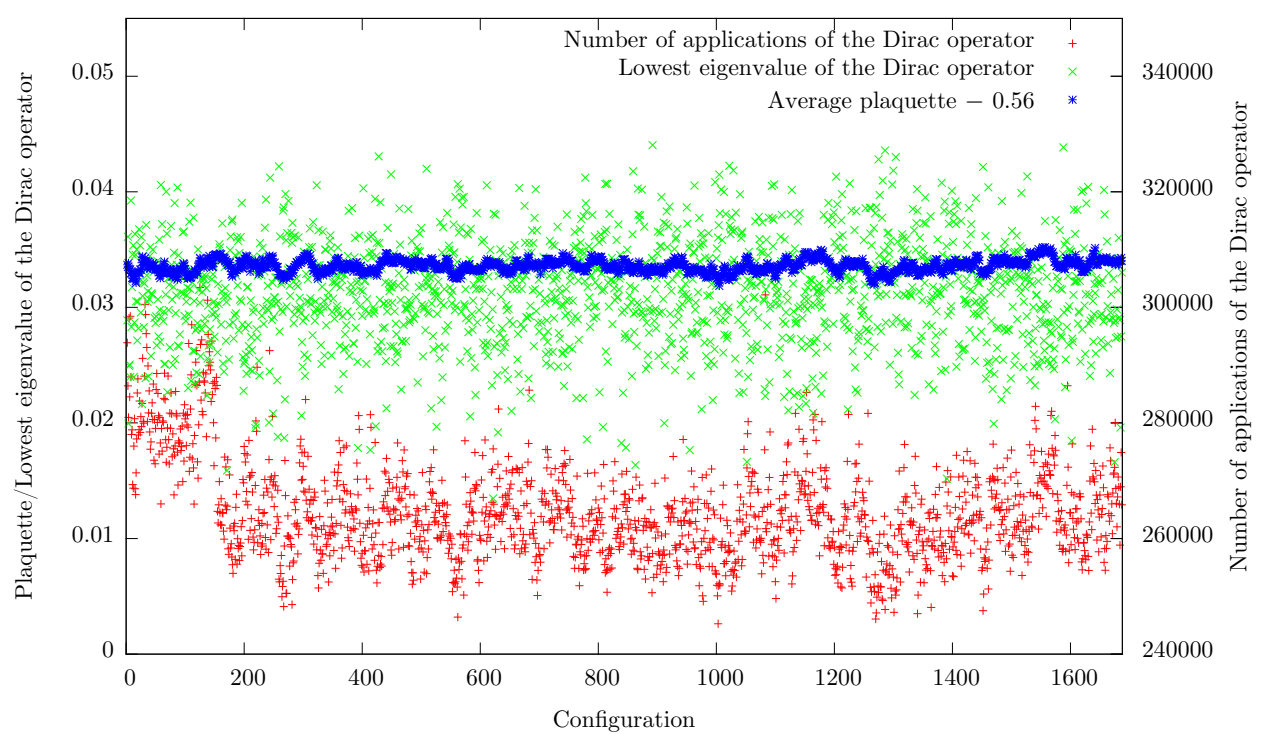

Figure 4.2: The run history for the D1 lattice; the plaquette is shifted vertically to improve the scaling on the $y$-axis.

An example run history for the RHMC is shown in figure 4.2. As would be expected, after an initial thermalisation period, the average plaquette, number of applications of the Dirac operator required, and the lowest eigenvalue of the Dirac operator all fluctuate around equilibrium values.

\subsubsection{Spectroscopy}

Spectroscopic observables measured were the masses of the pseudoscalar, scalar, vector, and axial vector mesons ${ }^{1}$, the spin- $\frac{1}{2}$ state $^{2}$, of the $0^{++}$glueball ${ }^{3}$; other mesonic states were found to be too noisy to get results from, and were deferred to a later study. Higher glueball states were found to have very poor statistics; this is not surprising, since glueballs are in general noisy observables. The spectroscopic results are summarised in figures 4.3 , where they are shown in lattice units, and 4.4, where they are given as

\footnotetext{
${ }^{1}$ The vector and axial vector were measured using a code that deals directly with Majorana fermions rather than translating from Dirac results.

${ }^{2}$ The spin-half state is a bound state of a gluon and a Majorana fermion; in supersymmetric theories it is referred to as the gluino-glue state. It is measured with an operator of the form $\sum_{\mu \nu} \sigma_{\mu \nu} \operatorname{tr}\left[F^{\mu \nu} \psi_{\mathrm{M} \pm}\right]$, where $\sigma_{\mu \nu}=\frac{1}{2}\left[\gamma_{\mu}, \gamma_{\nu}\right]$. It is also measured with the aforementioned code that works directly with Majorana fermions. [158]

${ }^{3}$ We expect that, having as they do the same quantum numbers, the $0^{++}$glueball and the scalar state will be the same asymptotic state; the two terms are used here to refer to the gluonic and mesonic operators used to measure them.
} 


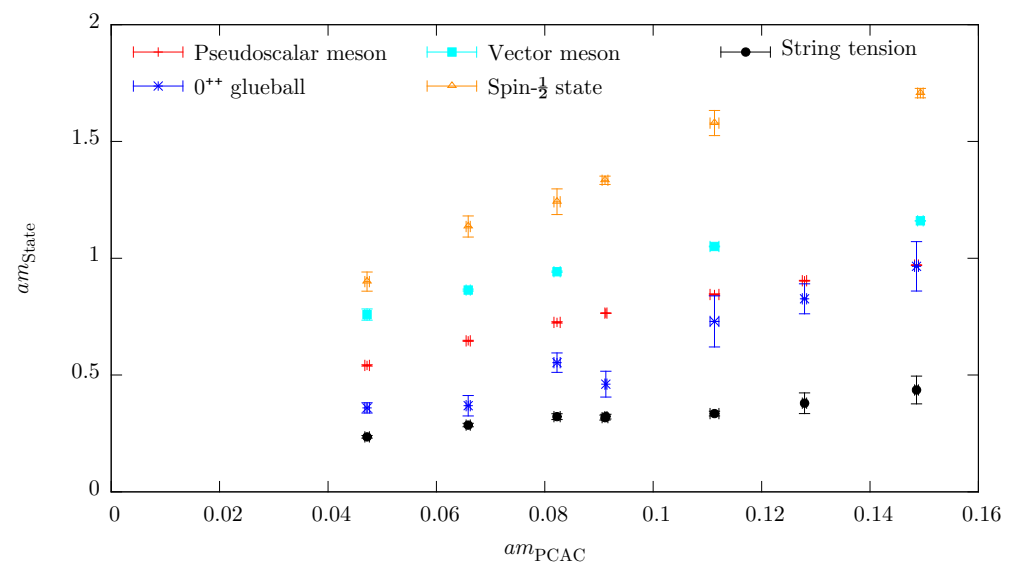

Figure 4.3: Raw spectroscopic results for the pseudoscalar and vector meson masses, the $0^{++}$glueball, the spin- $\frac{1}{2}$ state, and the fundamental string tension.

ratios with respect to the string tension; the raw data are shown in tables 4.4, 4.5, and 4.6. The string tension was calculated from correlators of Polyakov loops (also shown in table 4.5). Examples of the plateaux and cuts made for these variables are shown in figure 4.5. Topological observables were also considered; these are discussed in detail in chapter 5 .

The ratio of the pseudoscalar, vector, and axial vector meson masses to the string tension remain constant within $2 \sigma$ for the entire range observed. The spin- $\frac{1}{2}$ state behaves similarly, albeit with a slight rise at higher fermion masses. The $0^{++}$glueball has a mass comparable with the scalar meson near the chiral limit, while again lying slightly higher at higher fermion masses. The scalar meson is consistent with being the lightest state in the region in which it was calculated: the error bars are sufficiently large that the pseudoscalar is within two standard errors (or one, at higher fermion mass), so in principle one or two points could lie higher; however, the errors are statistical in nature, so the likelihood of all points lying at their current locations while having a "true" value above that of the pseudoscalar is extremely small.

To ensure that our study is not afflicted with finite-size artefacts, a set of runs were performed at all lattice sizes used, at $m=-1.51$, and their properties compared. The spectroscopy of this set of runs is shown in figure 4.6; we observe an overlap of the results from the three largest lattices, with the smallest lying a way away, indicating finite-size effects at this lattice size. 


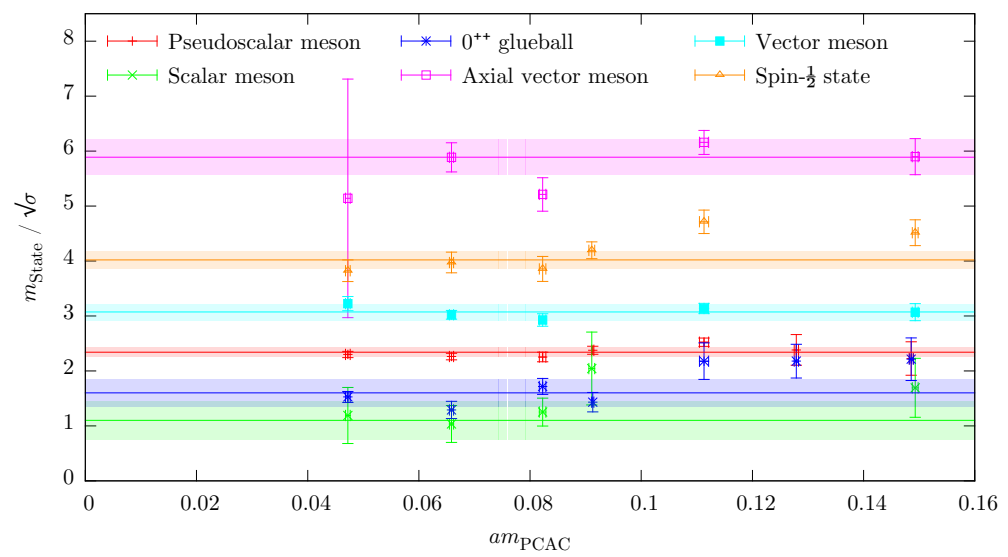

Figure 4.4: Spectroscopic results, showing the pseudoscalar, scalar, vector, and axial vector mesons, the spin- $\frac{1}{2}$ state, and the $0^{++}$glueball, normalised by the string tension, as a function of the PCAC fermion mass. At each bare fermion mass, only the data from the largest available lattice is shown (with the exception of the scalar state at $m=-1.51$, where the $\mathrm{B} 3$ results are used over the $\mathrm{C} 4$ results due to significantly lower errors). Approximate plateaux are also shown.

Since we now know where finite-volume effects begin to exhibit themselves in the case of one $m$ (and hence $\sigma$ ), we may use the condition that $L \sqrt{\sigma}$ (the normalised lattice extent) remains constant to constrain our lattice volumes at other values of $m$. This constraint excludes all of the $16 \times 8^{3}$ lattices from consideration, since they all fall in the region affected by finite-volume artefacts.

\subsubsection{Static potential from Wilson loops}

The configurations from this set of runs were also used (along with the D2 data) to analyse the static fermion potential, via Wilson loop calculations; the set of potentials found is shown in figure $4.7 \mathrm{a}$, with an example of a fit of the data shown in figure $4.7 \mathrm{~b}$. The results for the A4, C4, and D1 data overlap; the B3 data meanwhile are displaced by $\sim 0.2$. Since the $16 \times 8^{3}$ data are believed to be afflicted by finite size artefacts, the displaced case is an edge case so a disagreement is less worrying than it would be in, for example, the $\mathrm{C} 4$ data. However, the displacement can be entirely explained by the smearing used: since the smearing procedure was used on all links, rather than spacelike links only as some simulations use, the value for the potential obtained is 


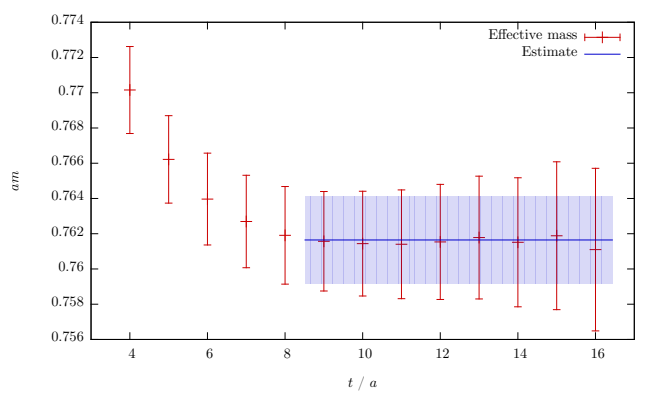

(a) The pseudoscalar meson.

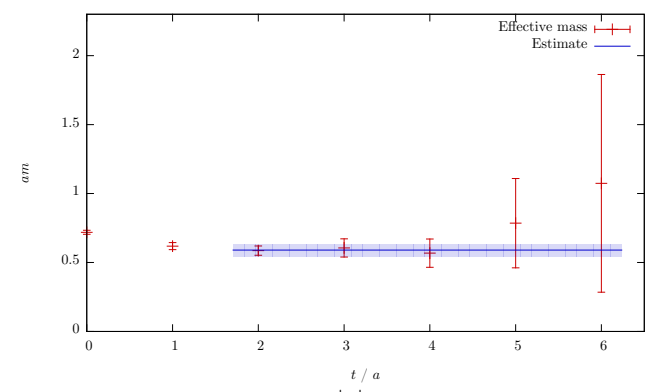

(b) The $0^{++}$glueball.

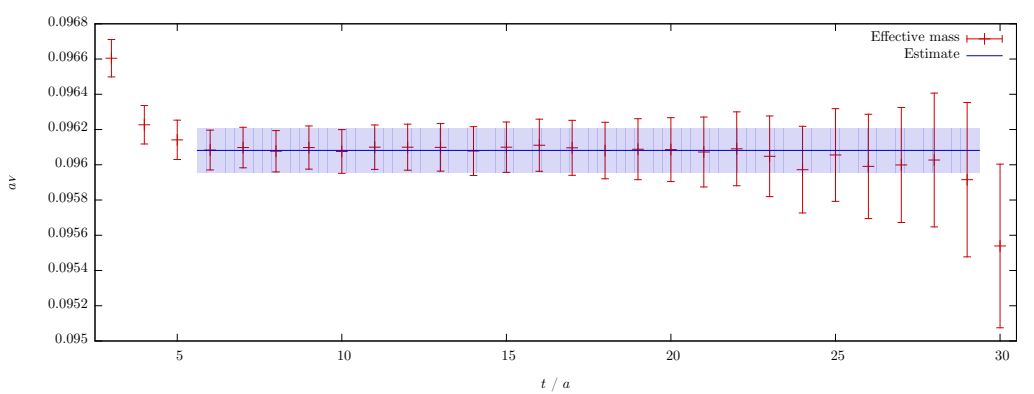

(c) The fermion potential in the $(1,0,0)$ direction.

Figure 4.5: Plateaux of various observables on the $32 \times 16^{3}$ lattice.

shifted. The data take the form

$$
V(r)=\alpha+\frac{\beta}{r}+\sigma r
$$

The factors $\alpha$ and $\beta$ are dependent on the smearing parameters chosen, causing a vertical shift and a distortion at the smallest $r$ values respectively; $\sigma$, however, is independent of the smearing. The $\mathrm{A} 4, \mathrm{C} 4$, and $\mathrm{D} 1$ data converged to similar values during the tuning process for the smearing parameters; the B3 data meanwhile ended in a different region. The output of the tuning program, and a comparison with another run using the smearing parameters of the $\mathrm{C} 4$ data (in which the results are no longer vertically displaced, and the errors are of a comparable size), suggest that there is a wide flexibility in choosing the tuning parameters with little change in the efficiency; this gives a solid reason for the different smearing results.

By fitting equation (4.84), the potentials may be used to extract an independent estimate for the string tension from that obtained via Polyakov loops; these are found to agree. This estimate is not affected by smearing artefacts, since the smearing only shifts 


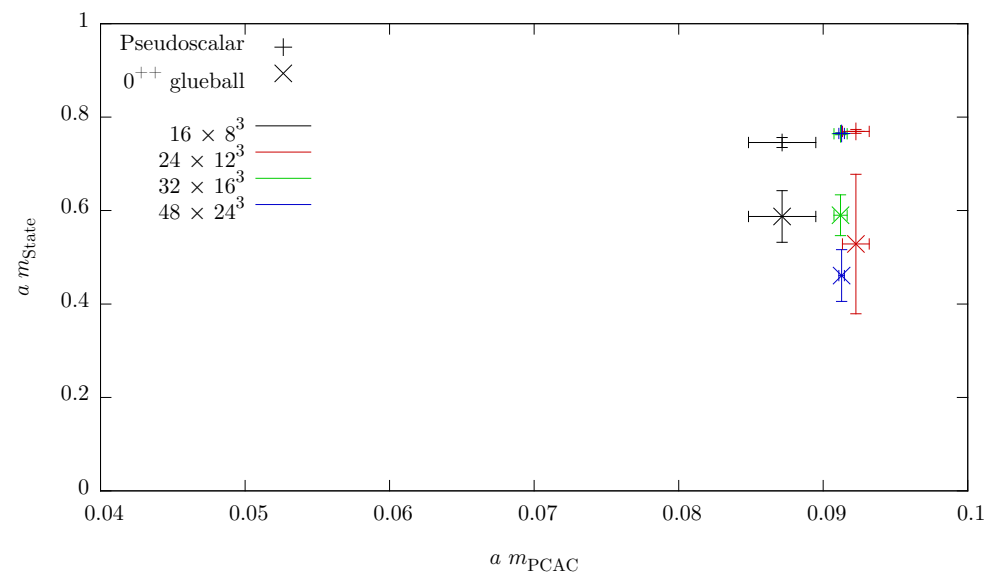

Figure 4.6: Spectroscopic results, showing the pseudoscalar meson and $0^{++}$glueball, for the finite-volume study.

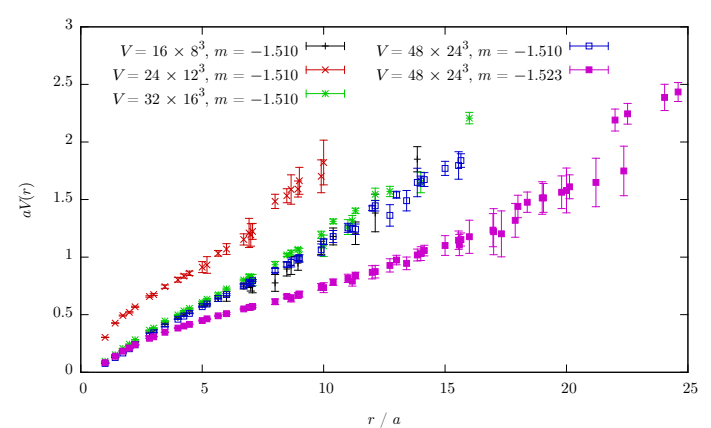

(a)

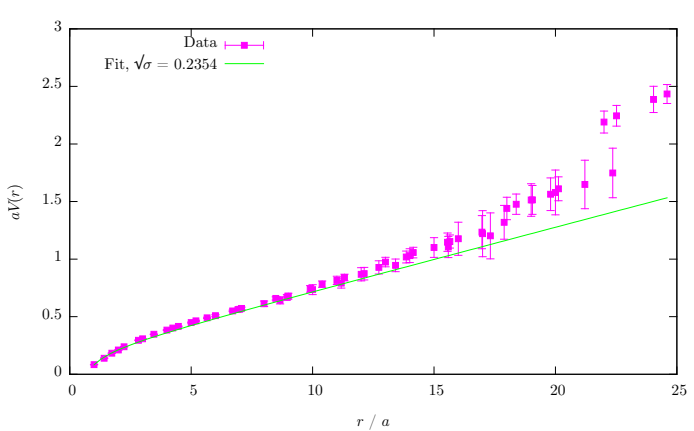

(b)

Figure 4.7: The static fermion potential, (a) for the four finite-size runs at $m=-1.51$ (A4, B3, C4, D1), and the D2 run at $m=-1.523$, and (b) for the D2 run only, with the fitted curve included.

the results vertically. Note in figure 4.7 that at high $r$ (in particular $r>L / 2$ ), the data cease to lie on the line suggested by the previous points. When fitting the effective mass at these points the plateaux are particularly short (often with two points and relatively large error bars), and so the result found may not be the true value. These points are included for information, but for fitting purposes the data were truncated to $r<L / 2$.

Adopting the results for the string tension from Polyakov correlators, and fitting the parameters $a$ and $b$ based on this value of $\sigma$, allows for an additional consistency check, and for stabilisation of the fits. The parameters obtained by fitting in this way are shown in table 4.7 , and an example is plotted in figure $4.7 \mathrm{~b}$. While the functional form 
is very close, the fit tends to lie below the data, reflecting a slight difference between the Wilson and Polyakov loop data. The systematic errors on the Wilson loop fits are yet to be fully quantified, so whether this discrepancy is within errors is unclear.

\subsubsection{Anomalous dimension from the Dirac mode number}

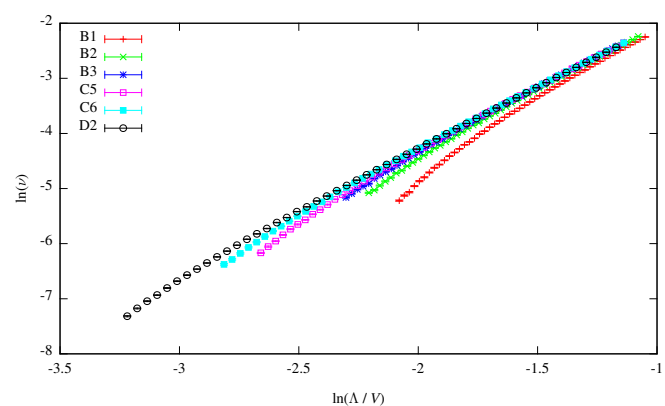

(a)

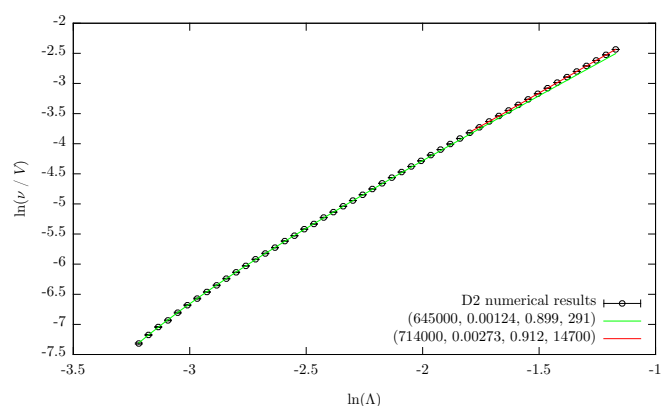

(b)

Figure 4.8: (a) The quantity $\bar{\nu} / V$ for the B1, B2, B3, C5, C6, and D2 lattices; (b) The results for the mode number from the D2 lattice, compared with two possible fit results.

The raw data for the mode number are shown in figure 4.8a. The results are consistent at high $\Omega$, but diverge at low $\Omega$, moving further from a straight line as the lattice volume is reduced. Whilst the mode number is a relatively quick observable to calculate, fitting it to obtain a value for the anomalous dimension $\gamma_{*}$ is less simple. Recall from section 3.2.4 that the mode number (in lattice units) takes the form

$$
a^{-4} \bar{\nu}(\Omega) \approx a^{-4} \bar{\nu}_{0}(m)+A\left[(a \Omega)^{2}-(a m)^{2}\right]^{\frac{2}{1+\gamma_{*}}}
$$

only in an intermediary region of $\Omega$. To account for this, we must consider all possible locations of the upper and lower end of this window when fitting the data. Additionally, the function is relatively insensitive to large correlated changes in the four fitted parameters $\bar{\nu}_{0}, A, m$, and $\gamma_{*}$-although $m$ is proportional to $m_{\mathrm{PCAC}}$, it must be fitted since we do not a priori know the constant of proportionality. This leads the fit to have a high sensitivity on initial conditions creating a systematic uncertainty on the fit parameters; the fitting algorithm will return a vastly underestimated value for the error on its output.

To overcome these problems, the first attempted fitting procedure takes the following approach. Each window of length 6 or more points (four parameters cannot 


\section{SU(2) WITH 1 DIRAC (2 MAJORANA) FLAVOURS}

reasonably be fitted with fewer than four points; four and five points were found to be sufficiently unstable that they were not considered) is considered in turn. For each window, an initial Levenberg-Marquardt fit $[159,160]$ is performed across the basic data set, with $\bar{\nu}_{0}=0$ set to allow convergence more easily. (Since convergence is still difficult even with this restriction, multiple attempts are made; the window is discarded after fifty unsuccessful attempts.)

The results of this fit are used as the basis for the initial conditions for the main fit. Then a set of 1000 bootstrap samples is created - each sample considers a random subset (with repetition permitted) of configurations, with the same subset considered for each point. Each sample is fitted, again with the Levenberg-Marquardt algorithm, with initial conditions given by an up to $10 \%$ uniform random offset from those given by the initial fit, and $\nu_{0} \in[0,1000)$ another random choice. If the fit fails to converge, then an inefficiency counter is incremented so that the quality of the fit can be judged. If the fit converges to an unreasonable value of $m$ (which must lie below the lower end of the window), the window is discarded - even if only one of the thousand samples behaves in this way, it is still indicative of the fit driving to the wrong regime. Once all 1000 samples have been considered, then the parameters for that window are reported as the means and standard errors of the sample fits. The inefficiency is also output as a percentage, as is the $\chi^{2}$ deviation per point.

We now have an output file with estimates of the fit parameters for each window. The number of possible windows is quite large, which leaves us in the unusual situation of needing to fit our fit results. While a colour map could be used to give an overall impression of the quality of the data an an initial impression of where the best regions lie and what the fitted parameters will be, they are poor presentational tools due to the difficulty that can be had in interpreting them, and they are not sufficiently quantitative for the data we are working with here.

To obtain quantitative estimates of the parameters, we must fix the upper and lower ends of the window in turn, and look for plateaux in their values. Plots are created for each fitted observable $\left(A, m^{2}, \gamma_{*}\right.$, and $\left.\bar{\nu}_{0}\right)$; a set of such plots is shown in figure 4.9. In each plot, a variety of possible lower ends of the window are considered, represented by different colours. (The lowest-lying data have been omitted here since they add only noise.) Then the upper end of the window is used to position the points on the $x$-axis, with the observable on the $y$-axis. 
Lower end of window:
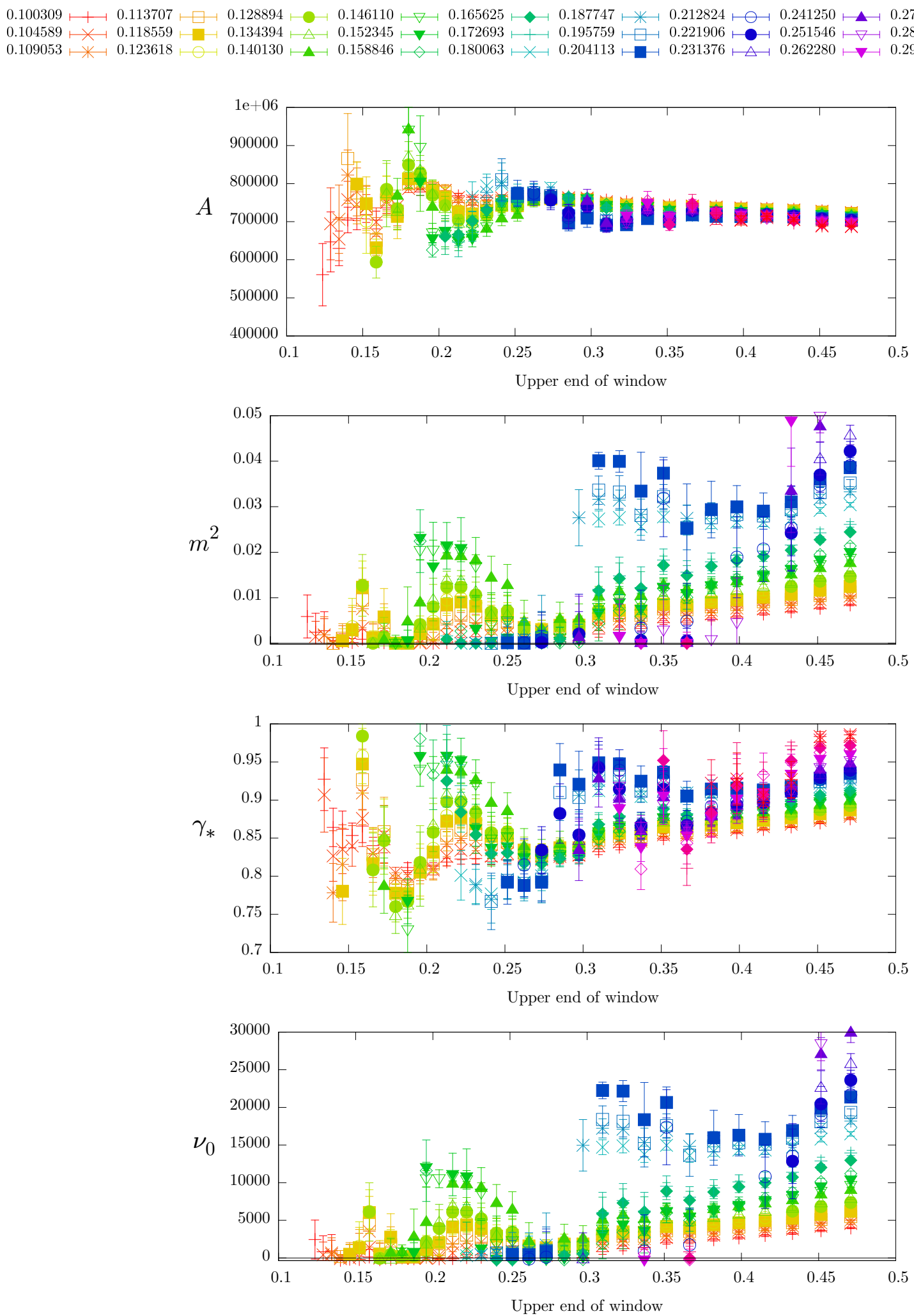

Figure 4.9: Plateaux for the fitted observables for the D2 data at various lengths and positions of the fitting window. The colour represents the position of the lower end of the window, and the $x$-axis the upper end. 


\section{SU(2) WITH 1 DIRAC (2 MAJORANA) FLAVOURS}

In the region of the physical values, we expect to see plateaux in many nearby upper and lower ends of the window (since a slight adjustment of the window position or length should not affect the fit), and in the same region in all observables. On the plot, this corresponds to plateaux in nearby colours, at similar vertical positions within the individual plots, and at the same horizontal ranges on all plots.

When this is done, plateaux are seen for upper ends between $0.3 \lesssim \Omega \lesssim 0.5$, with the lower end in the range $0.2 \lesssim \Omega \lesssim 0.25$ (the blue region of the spectrum). This gives an anomalous dimension in the range $0.9 \lesssim \gamma_{*} \lesssim 0.95$. However, it is possible that the Levenberg-Marquadt algorithm has been biased in some way due to the difficulty of the data. To verify whether this is the case, it seems prudent to attempt a second, independent fitting method to cross-check the consistency of the results found.

A suitable alternative, which is well-suited to situations where the system may converge to a local minimum, is known as simulated annealing [161, 162]. This method is functionally the same as a Monte Carlo simulation, where the action is replaced by $\chi^{2}$ for the parameters. The use of the Metropolis algorithm allows the fit to move away from local minima in $\chi^{2}$ to find the global minimum. The "annealing" occurs when the temperature of the system is gradually reduced, slowly reducing the Metropolis's ability to move away from peaks. This forces the fit to converge on the global minimum, rather than continuing to sample the whole distribution. As in the previous approach, this is done for each possible window in turn. This was done by a collaborator, and the results were found to be consistent with the results from Levenberg-Marquadt.

\subsection{Conclusions}

We have carried out the first look at SU(2)Adj Nf1 on the lattice. After an initial exploratory study of the phase structure in the $\beta-m$ plane, quantitative studies were made at a variety of lattice sizes (with a single lattice spacing), looking at the meson and glueball spectrum, the static potential and string tension from Wilson and Polyakov loop correlators, and the anomalous dimension of the chiral condensate. (The topology is also considered in the next chapter.) In the spectrum we observe that ratios of spectral quantities are constant over a wide range of fermion mass, which is consistent with behaviour that is not "traditionally" confining (i.e. either conformal, or confining but near the lower edge of the confomal window) - in particular, the non-vanishing of 
the pion mass as $m_{\mathrm{PCAC}} \rightarrow 0$. The rise in non-mesonic state masses at higher fermion mass may be a statistical anomaly, or a large-mass effect.

The theory also shows signs of admitting a light scalar state, supporting the finding of non-QCD-like behaviour. While a confining scenario with adjoint matter could account for the observation that the mesons lie above the lightest glueball state, we would still expect the pseudoscalar to be the lightest mesonic state, in contrast to the observation here that the scalar meson lies below the pseudoscalar. While the errors are sufficiently large that a single data point could be conjectured to be misordered, the presence of multiple data points two standard errors below the pseudoscalar makes such a conclusion unsupportable.

The anomalous dimension is tentatively found to be in the region $0.9 \lesssim \gamma_{*} \lesssim 0.95$, which is one of the highest anomalous dimensions found in a lattice gauge theory. Were it not for the insufficient number of Goldstone states from $\chi \mathrm{SB}$ discussed in section 3.4, this would be an excellent candidate theory for Technicolor phenomenology; as it stands it is still very interesting, since it implies the conformal window for $\mathrm{SU}(2)$ with adjoint matter may extend lower than is expected from approximate analytic methods.

It would be interesting to extend the study to lower masses (via larger lattice sizes), to probe the behaviour closer to the chiral limit. Probing higher mass to investigate whether the rise in non-mesonic spectral quantities is a statistical artefact requires smaller lattice spacing; this is also necessary to find the continuum limit of these results, which is another important next step. This will also allow us to be $100 \%$ certain that we are not simulating lattice artefacts - as we have simulated only a single value of $\beta$, although we have been careful to avoid the bulk phase, the possibility remains that lattice artefacts remain. Finally, since $N_{\mathrm{f}}=1$ (two Majorana flavours) has too few and $N_{\mathrm{f}}=2$ (four Majorana flavours) too many Goldstone states, and both lie within or near the conformal window, it seems a good idea to perform a similar study of the theory with three Majorana flavours (which lies between the two). 
4. SU(2) WITH 1 DIRAC (2 MAJORANA) FLAVOURS 


\section{Chapter 5}

\section{Topology}

There exist topological objects in classical and quantum field theories called instantons. They are non-perturbative gauge field configurations, which correspond to tunnelling events between vacua. Their interactions with quarks have been found to result in chiral symmetry breaking, and it has therefore been suggested that instantons play some rôle in deconfinement.

In this chapter I will present the results of a study of the topology of two theories: SU(2)Adj Nf1 and Minimal Walking Technicolor (MWT). Both theories are outlined in section 3.4, and more details of the study of SU(2)Adj Nf1 are in chapter 4. In section $5.1 \mathrm{I}$ will describe instantons and their properties, while in section $5.2 \mathrm{I}$ will outline the apparatus for observing and calculating properties of instantons on the lattice. In section $5.3 \mathrm{I}$ will present the results of studies of instantons in the two $\mathrm{SU}(2)$ theories mentioned, and in the $\mathrm{SU}(2)$ pure gauge theory for comparison, which are then discussed in section 5.4. Concluding remarks are given in section 5.5.

\subsection{Instantons}

In this section I will follow the presentation of Schäfer and Shuryak's review [9]. We start by looking at the classical vacua of Yang-Mills theory. The Hamiltonian under consideration is

$$
H=\frac{1}{2 g^{2}} \int d^{3} x\left(E_{i}^{2}+B_{i}^{2}\right),
$$

where the electric field $E=\partial_{0} A_{i}$ forms the kinetic term, and the magnetic field $B$ gives the potential term. The classical vacua have zero field strength, and can be obtained 


\section{TOPOLOGY}

by a gauge transformation from the trivial vacuum, giving

$$
A_{i}=i U(\mathbf{x}) \partial_{i} U(\mathbf{x})^{\dagger}
$$

Enumerating the vacua involves classifying all possible gauge transformations $U(\mathbf{x})$, which in turn requires studying equivalence classes of maps from $\mathbb{R}^{3}$ into $\mathrm{SU}(N)$. We can limit this set of maps to those obeying $U(\mathbf{x}) \rightarrow 1$ as $x \rightarrow \infty$ [163]. These mappings can be classified by their winding number, an integer defined as

$$
n_{\mathrm{W}}=\frac{1}{24 \pi^{3}} \int d^{3} x \epsilon^{i j k} \operatorname{tr}\left[\left(U^{\dagger} \partial_{i} U\right)\left(U^{\dagger} \partial_{j} U\right)\left(U^{\dagger} \partial_{k} U\right)\right]
$$

This is none other than the Chern-Simons characteristic:

$$
n_{\mathrm{CS}}=\frac{1}{16 \pi^{2}} \int d^{3} x \epsilon^{i j k}\left(A_{i}^{a} \partial_{j} A_{k}^{a}+\frac{1}{3} f^{a b c} A_{i}^{a} A_{j}^{b} A_{k}^{c}\right) .
$$

In the case of $\mathrm{SU}(2)$, if we wish to find an example of a mapping with a given winding number $n$, we can make use of the hedgehog ansatz, which states

$$
U(\mathbf{x})=\exp \left(i f(r) \tau^{a} \hat{x}^{a}\right)
$$

where $r=|\mathbf{x}|, \hat{x}^{a}=x^{a} / r$. Substituting this in shows

$$
n_{\mathrm{W}}=\frac{1}{\pi}\left[f(r)-\frac{\sin (2 f(r))}{2}\right]_{0}^{\infty} .
$$

If we constrain $U(\mathbf{x})$ to be uniquely-defined, then $f(r)$ must be an integer multiple of $\pi$ at $r=0, \infty$, so we can see trivially from equation (5.6) that $n_{\mathrm{W}}$ is then an integer. Then any smooth $f(r)$ with $f(r \rightarrow \infty)=0, f(0)=n \pi$ provides an example of a function with winding number $n_{\mathrm{W}}=n$.

We have thus found an infinite number of classical vacua labelled by an integer $n$, which are topologically distinct: we cannot go from one to another by a continuous gauge transformation, and there is no path from one to another such that the energy remains zero all the way along. Quantum mechanically, though, we are aware that we may tunnel between vacua. To see this here, we look for paths with minimum Euclidean action connecting vacua with different winding number. Starting with the action in the 
form:

$$
\begin{aligned}
S & =\frac{1}{4 g^{2}} \int d^{4} x F_{\mu \nu}^{a} F_{\mu \nu}^{a} \\
& =\frac{1}{4 g^{2}} \int d^{4} x\left[ \pm F_{\mu \nu}^{a} \tilde{F}_{\mu \nu}^{a}+\frac{1}{2}\left(F_{\mu \nu}^{a} \mp \tilde{F}_{\mu \nu}^{a}\right)^{2}\right]
\end{aligned}
$$

where $\tilde{F}_{\mu \nu}=\frac{1}{2} \epsilon_{\mu \nu \rho \sigma} F_{\rho \sigma}$ is the dual field strength tensor, in which the rôles of electric and magnetic field have been reversed. It is evident that $S$ is extremised when the field is either self-dual or anti-self-dual:

$$
F_{\mu \nu}^{a}= \pm \tilde{F}_{\mu \nu}^{a}
$$

From this we define the topological charge

$$
Q=\frac{1}{32 \pi^{2}} \int d^{4} x F_{\mu \nu}^{a} \tilde{F}_{\mu \nu}^{a}
$$

which we may rewrite in the form

$$
Q=\int d^{4} x \partial_{\mu} K_{\mu}=\int d \sigma_{\mu} K_{\mu}
$$

where

$$
K_{\mu}=\frac{1}{16 \pi^{2}} \epsilon_{\mu \alpha \beta \gamma}\left(A_{\alpha}^{a} \partial_{\beta} A_{\gamma}^{a}+\frac{1}{3} A_{\alpha}^{a} A_{\beta}^{b} A_{\gamma}^{c}\right) .
$$

Provided that $A_{\mu}$ drops off sufficiently rapidly at spatial infinity, then

$$
Q=\int d t \frac{d}{d t} \int d^{3} x K_{0}=n_{\mathrm{CS}}(t=\infty)-n_{\mathrm{CS}}(t=-\infty) .
$$

Thus if $Q \neq 0$ then the field configuration connects topologically distinct vacua at different times. Such a solution is referred to as an instanton.

The first such solution reported was found by Belavin, Polyakov, Schwartz, and Tyupkin [164], and is hence called the BPST instanton. BPST referred to it as a "pseudoparticle", with the name "instanton" only being coined later. Its gauge potential takes the form

$$
A_{\mu}^{a}(x)=\frac{2 \eta_{a \mu \nu} x_{\nu}}{x^{2}+\rho^{2}}
$$


where $\eta_{a \mu \nu}$ is the 't Hooft symbol

$$
\eta_{a \mu \nu}= \begin{cases}\epsilon_{a \mu \nu} & \mu \nu=1,2,3 \\ \delta_{a \mu} & \nu=4 \\ -\delta_{a \nu} & \mu=4\end{cases}
$$

and $\rho$ is an arbitrary parameter characterising the instanton size. This instanton carries topological charge $Q=1$. A variant with $Q=-1$ is found by replacing $\eta$ with $\bar{\eta}$, which swaps $\delta \leftrightarrow-\delta$.

The form of the instanton in equation (5.14) proves inconvenient to work with; we may make a gauge transformation to singular gauge to give a gauge potential of the form:

$$
A_{\mu}^{a}(x)=2 \frac{x_{\nu}}{x^{2}} \frac{\bar{\eta}_{a \mu \nu} \rho^{2}}{x^{2}+\rho^{2}} .
$$

This is singular at the origin, but the singularity is not physical. It does however localise the topology to the origin, which allows easier study of instanton-anti-instanton configurations.

Instantons also play a rôle in chiral symmetry breaking; they solve the $\mathrm{U}(1)$ problem, by breaking the chiral U(1) symmetry, which would otherwise break spontaneously to give additional pions not observed in nature [165]. It is possible to model the vacuum of QCD (or another gauge theory) as a dense liquid of instantons, in what is aptly referred to as the "instanton liquid model". This framework allows computation of some non-perturbative observables (within the limits placed by the oversimplifications of the model, which neglect all other degrees of freedom) beyond perturbation theory without the need for lattice computations; the lattice is still however necessary both to confirm the results of at least some of these computations (to verify the method) and to go beyond the limitations of the model. Recent examples of instanton liquid model calculations include studies of the QCD magnetic susceptibility [166] and of the QCD electrical conductivity [167].

\subsection{Methodology}

The lattice is set up as described in section 3.1. The observables studied are described in detail in [168]; here, I will summarise the main points. In the continuum, from 
equation (5.10) the topological charge density can be expressed as

$$
Q(x)=\frac{1}{32 \pi^{2}} \epsilon_{\mu \nu \rho \sigma} \operatorname{tr}\left\{F_{\mu \nu}(x) F_{\rho \sigma}(x)\right\}
$$

The total topological charge of a configuration can then be expressed as the space-time integral of this quantity.

The equivalent lattice topological charge density is then given by:

$$
Q_{\mathrm{L}}(x)=\frac{1}{32 \pi^{2}} \epsilon_{\mu \nu \rho \sigma} \operatorname{tr}\left\{U_{\mu \nu}(x) U_{\rho \sigma}(x)\right\}
$$

For a smooth gauge field, this would appear to have fluctuations of order $a^{2}$ as the lattice spacing $a$ is sent to zero; however, realistic fields have ultraviolet fluctuations which will completely dominate over the physics of interest in the continuum limit. To mitigate this, a cooling process is introduced [169]. The cooling process operates by minimising the local action for each lattice site in turn.

Successive cooling sweeps will "smooth out" the fluctuations such that the semiclassical physics may be observed. A side effect however is that instantons may be shrunk or may annihilate in instanton-anti-instanton pairs, thus excessive cooling is to be avoided. The number of cooling sweeps used in a calculation is chosen as a compromise between the need to smooth out the configurations and the requirement to not annihilate physical instantons. There is however a wide plateau where the physics can be observed without cooling artefacts becoming problematic, so the number of cools need not be finely tuned.

Once the topological charge density is known, various observables may be calculated. $Q_{\mathrm{T}}$ is the total topological charge, defined by

$$
Q_{\mathrm{T}}=\sum_{i} Q_{\mathrm{L}}(i)
$$

with $i$ running over all lattice points. Since the continuum topological charge is an integer, the lattice $Q_{\mathrm{T}}$ is often rounded to the nearest integer. Lattice studies (e.g. [170]) show that when working close to the continuum limit, the particular choice of definition for $Q_{\mathrm{T}}$ does not affect the results obtained - as we would expect, since any differences should be lattice artefacts, which will disappear in powers of the lattice spacing. Armed with the total topological charge, we may then proceed to find the 


\section{TOPOLOGY}

topological susceptibility, which is defined as

$$
\chi_{\mathrm{T}}=\frac{\left\langle Q_{\mathrm{T}}^{2}\right\rangle}{V}
$$

where $V$ is the lattice volume. This form, however, introduces systematic errors which may be removed by instead calculating

$$
\chi_{\mathrm{T}}=\frac{\left\langle Q_{\mathrm{T}}^{2}\right\rangle-\left\langle Q_{\mathrm{T}}\right\rangle^{2}}{V},
$$

since $\left\langle Q_{\mathrm{T}}\right\rangle$ is expected to be zero, so any difference from zero would be due to an additive constant.

The size of a given instanton may be calculated from the local maxima and minima of the topological charge density from the relation

$$
q_{\text {peak }}=\frac{6}{\pi^{2} \rho^{4}},
$$

which can be derived by substituting the gauge potential for the BPST instanton (equation (5.16)) into the definition of the topological charge density (equation (5.17)). This is the method that was used to obtain the results presented below. It is possible to introduce lattice corrections to this form, and to account for the effect that the overlap between instantons will have on the peak topological charge density; these methods have not been used for this work.

\subsection{1 $\mathrm{SU}(N)$ results}

To allow an informed comparison between the results found during this research and prior work, some examples from the literature are quoted here. Figure 5.1 shows the topological susceptibility for $\mathrm{SU}(N)$ pure gauge theories, $N=2,3,4,5$ (plotted as $\chi^{\frac{1}{4}} / \sqrt{\sigma}$ against $a^{2} \sigma$ ), with straight line behaviour observed for each case as $a \rightarrow 0$. Figure 5.2 shows the instanton size distributions for $\mathrm{SU}(2)$ and $\mathrm{SU}(3)$, showing a singlepeaked distribution in all cases, falling to zero at $\rho \rightarrow 0$ and $\rho \rightarrow \infty$. We will look for similar patterns in our data, and try to explain any observed differences. 


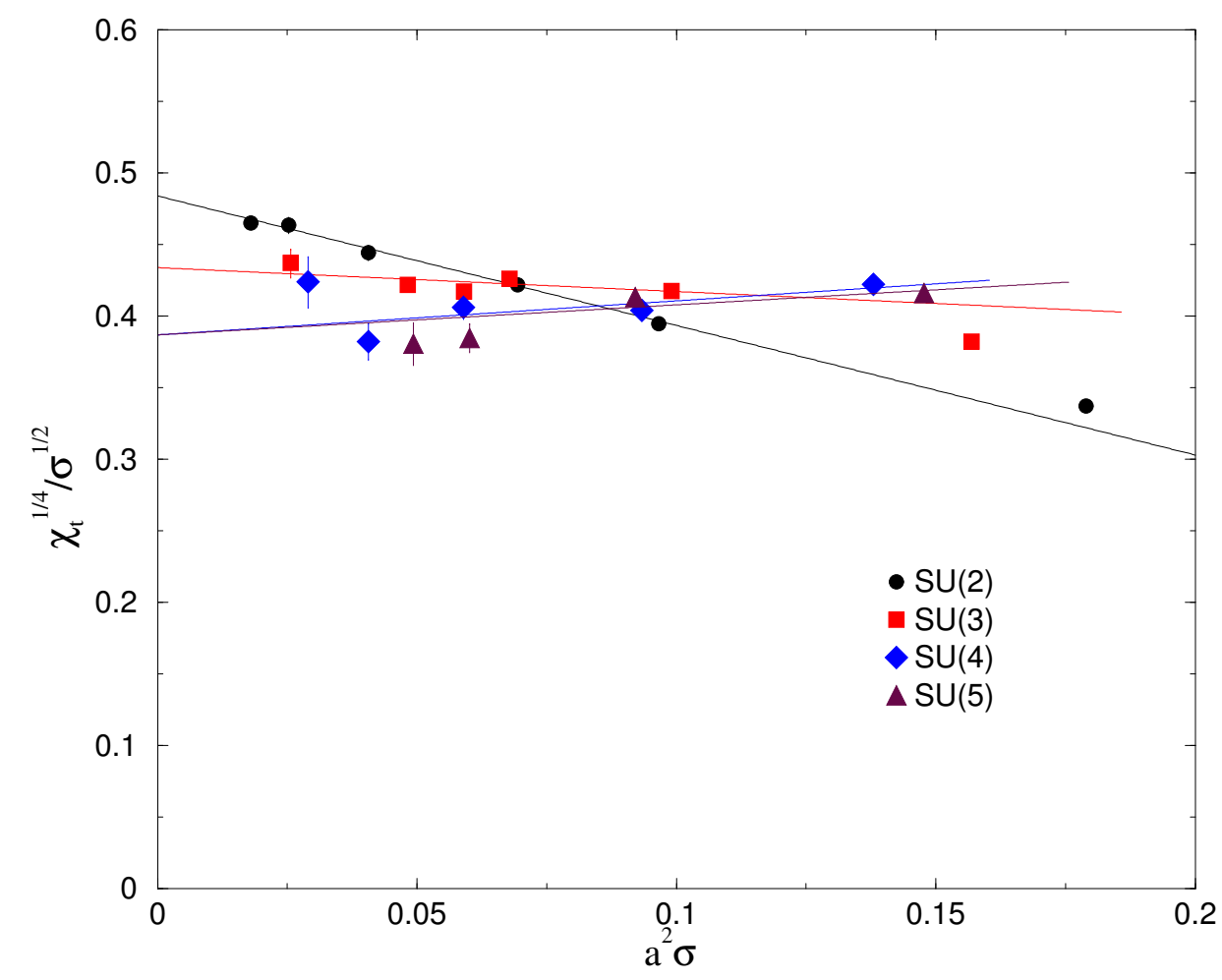

Figure 5.1: Variation of the quantity $\chi^{\frac{1}{4}} / \sqrt{\sigma}$ with $\sigma$ for $\mathrm{SU}(n)$ theories, from [8].
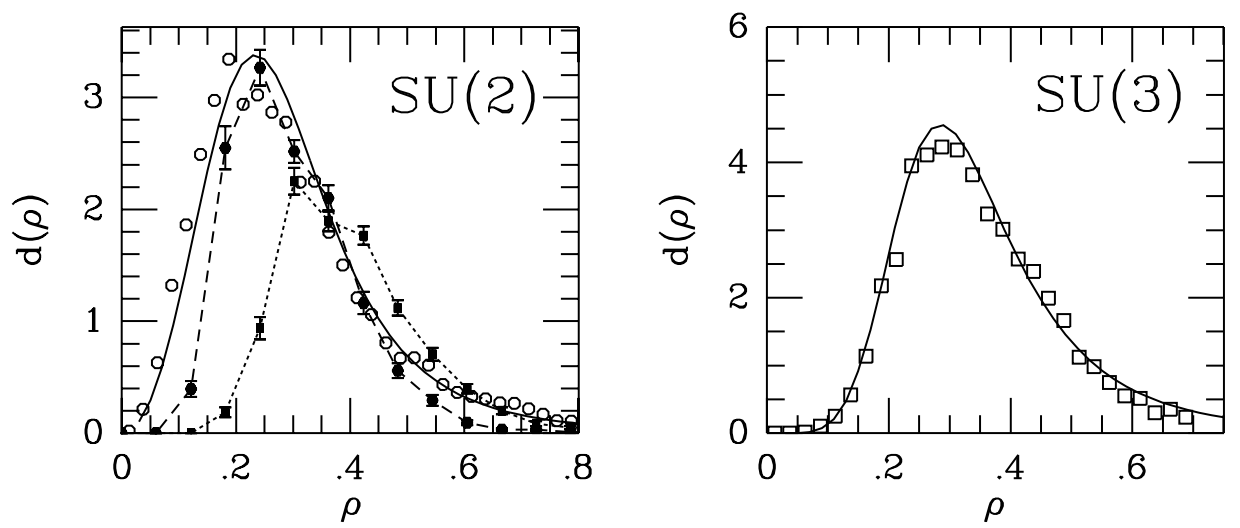

Figure 5.2: Instanton size distributions for $\mathrm{SU}(2)$ and $\mathrm{SU}(3)$, from [9]. 


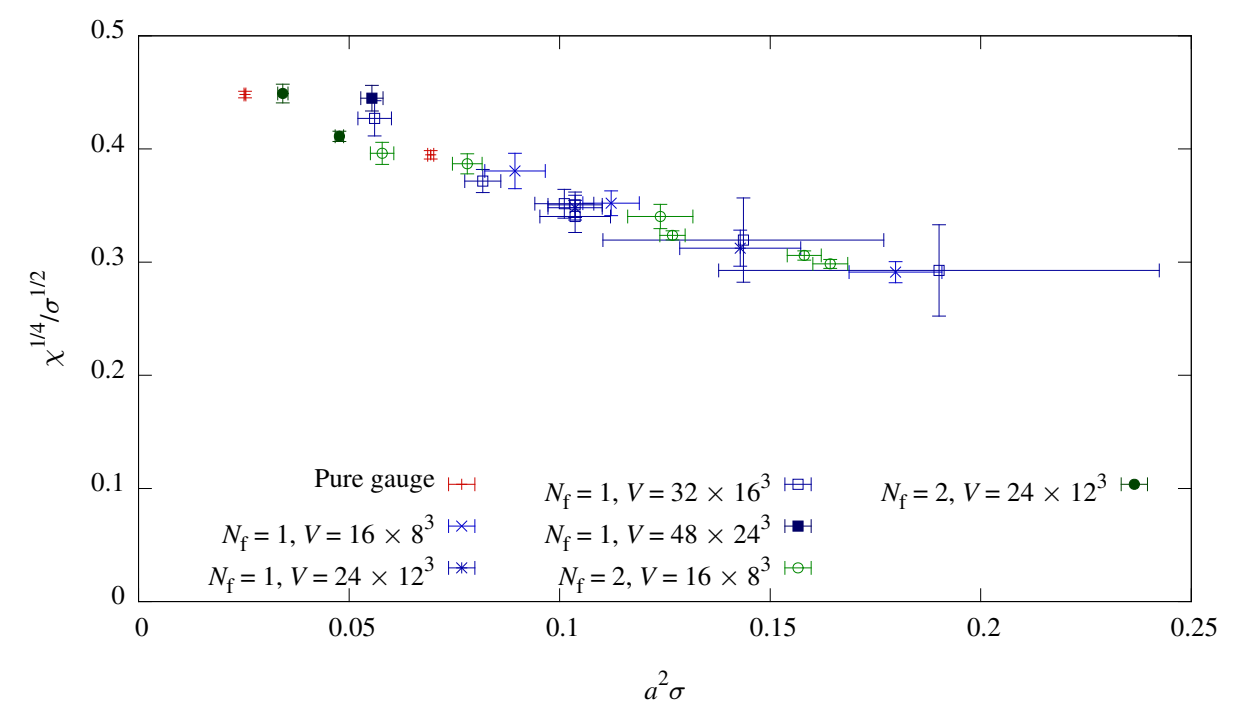

Figure 5.3: Variation of the quantity $\chi^{\frac{1}{4}} / \sqrt{\sigma}$ with $\sigma$.

\subsection{Numerical Results}

Computations were carried out on an x86-64 cluster and a Mac Pro, both in Swansea University. Two-flavour configurations prepared for ongoing work (discussed in [10], where measurements of spectral quantities, and analysis of finite volume artefacts, may be found) were used, at $\beta=2.25$, with $V=16 \times 8^{3}, m=-0.90,-0.75,-0.50,-0.25$, $0.00,0.25,0.50$ and $V=24 \times 12^{3}, m=-1.00,-0.95$. The one-flavour configurations discussed in Chapter 4 were also used; details of the combinations of parameters and lattices used are given in that chapter.

To allow comparison with the pure Yang-Mills case, pure gauge configurations were generated at $\beta=2.4, V=12^{4}$ and $\beta=2.55, V=20^{4}$. Each update consisted of 1 heat bath and 4 over-relaxed steps, and measurements were taken every 10th and 50th configuration on the smaller and larger lattice respectively. For each $\beta$, the lattice size was chosen such that finite size effects were negligible. 20 cooling sweeps were used for all configurations.

The findings (shown in figure 5.3) show consistency between the three cases, and both qualitatively and quantitatively with Lucini and Teper's results in figure 5.1 [8].

The topological susceptibility shows no sign of finite-size effects. However, the instanton size distribution shows a different picture. In the case of the pure gauge theory at $V=12^{4}$ and the 1- and 2-flavour cases at $V=24 \times 12^{3}$ and larger (shown in figure 


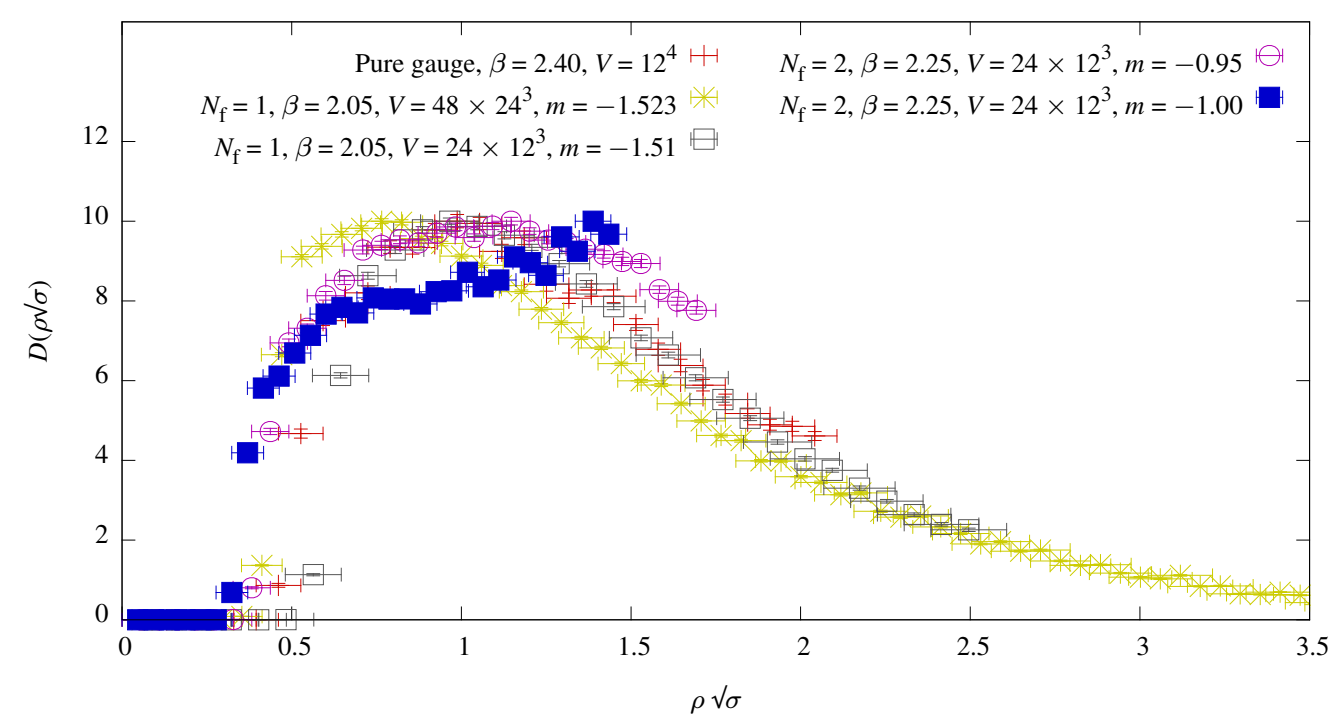

Figure 5.4: Instanton size distribution for one- and two-flavour configurations on the $24 \times 12^{3}$, for one-flavour configurations on the $48 \times 24^{3}$ lattices, and for the pure gauge configurations. In all cases the distributions have been truncated at $\rho=\frac{2}{3} L_{T}$ to avoid boundary effects.

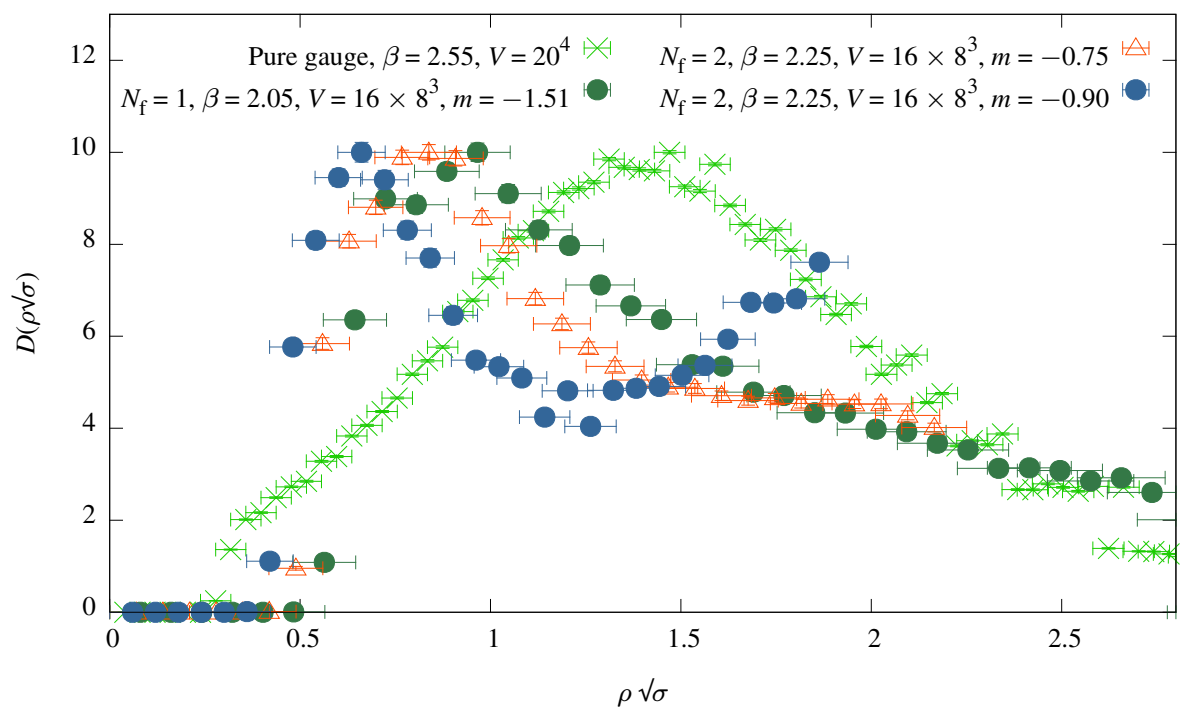

Figure 5.5: Instanton size distribution for some of the one- and two-flavour runs on the $16 \times 8^{3}$ lattice, exhibiting severe finite-size effects, and the $20^{4}$ pure gauge data, exhibiting cooling artefacts at small instanton size. 


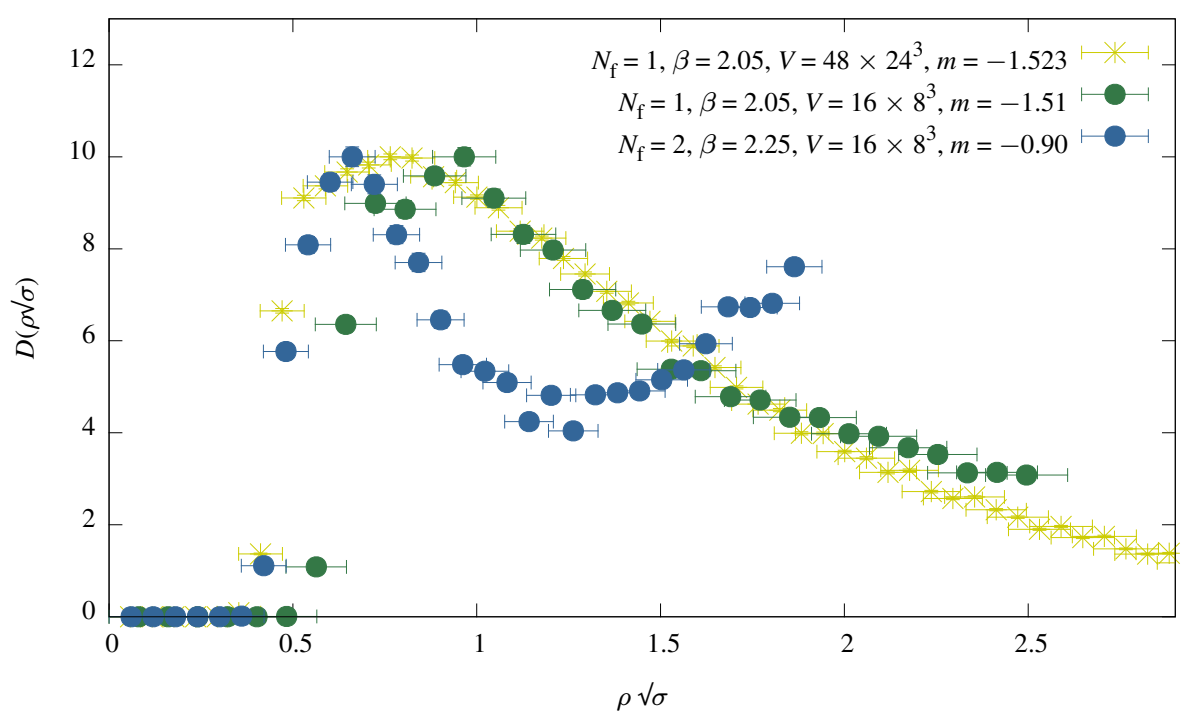

Figure 5.6: Comparison between the instanton size distributions of one- and two-flavour configurations affected and not affected by finite-size effects.

5.4), the distributions are consistent both with each other and with the distribution found in similar pure-gauge studies [171-173] (beyond, that is, the start of the distribution, which is controlled by the lattice spacing). However, looking at the $16 \times 8^{3}$ lattice, the two-flavour theory shows a long tail in the instanton size distribution, which grows into a second peak in the region where finite-size effects are encountered. The initial peak is also distorted. The distortion in the tail is best seen in the region cut off in the previous plot (where we expect finite-size effects to be strongest); this region is included in figures 5.5 and 5.6. In figure 5.5, some examples of finite-size-effect-afflicted distributions are shown; in figure 5.6, some examples are compared to a non-afflicted distribution.

All else being equal, this would cause the average instanton size to lie above the true value; however, in figure 5.7 we see that on the smaller lattices $\bar{\rho}$ lies below the value on larger lattices. This is due to the tail of the distribution being truncated. Two estimates for the average instanton size are made: one considering only $\rho<\frac{2}{3} L$ (since instantons larger than this may be distorted by being close to the box size), and one considering all $\rho<L$ (since $\rho>L$ is obviously spurious, since such an instanton could not fit on the lattice). Since the former approximation truncates a significant amount of the tail of the distribution in most cases, an estimate of the error this introduces is made by 


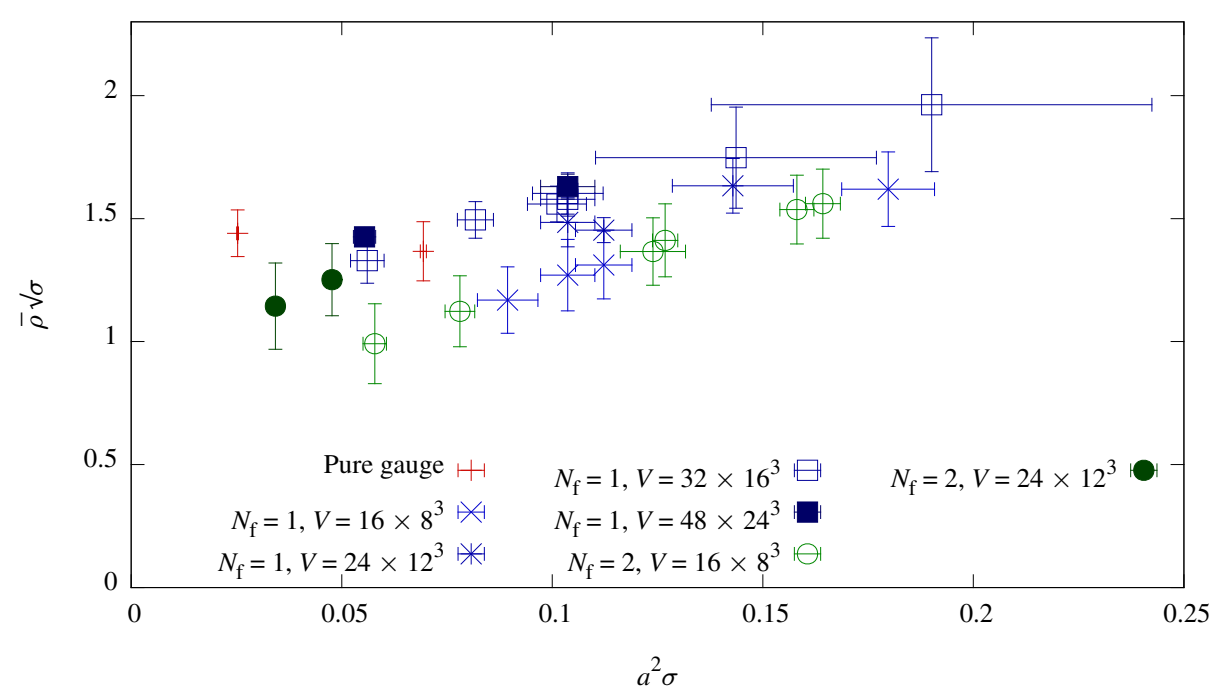

Figure 5.7: Variation of the average instanton size with $\sigma$.

placing the point with sufficiently large errors that both estimates are included. In the smallest lattices, even the latter truncation still removes much of the tail, which when combined with the aforementioned finite-size distortions to the distribution, cause the observed reduction of the average size. Whilst it might be possible to correct for this, the other distortions lead it to be not worthwhile.

The topological charge distribution was also computed for a single configuration of a $64 \times 32^{3}$ lattice for $\mathrm{SU}(2) \operatorname{Adj} \mathrm{Nf1}$, with $\beta=2.05, m=-1.51$, and visualised in three dimensions using the VisIt visualisation tool $[174,175]$. The resulting graphics are shown in appendix A.

For a sufficiently large lattice, we expect that the topological susceptibility and average instanton size should scale as

$$
\begin{aligned}
& \left.\chi=L^{-4} F_{\chi}\left(L m^{1 /\left(1+\gamma_{*}\right)}\right)\right), \\
& \bar{\rho}=L F_{\bar{\rho}}\left(L m^{1 /\left(1+\gamma_{*}\right)}\right),
\end{aligned}
$$

where $\gamma_{*}$ is the anomalous dimension of the (chiral) condensate. Attempts were made to fit both the SU(2)Adj Nf1 and the MWT results to these relation by hand (as described in [10]), without success. A representative sample of the plots generated for this fit are shown in figures 5.8-5.11. 


\begin{tabular}{cccccc}
\hline Lattice & $a L$ & $a m_{0}$ & $a m_{\mathrm{PCAC}}$ & $a^{4} \chi$ & $\bar{\rho} / a$ \\
\hline $\mathrm{A} 1$ & 8 & 1.475 & $0.1489(9)$ & $2.321(85) \times 10^{-4}$ & $3.82(34)$ \\
$\mathrm{A} 2$ & 8 & 1.500 & $0.1101(12)$ & $1.935(69) \times 10^{-4}$ & $3.91(39)$ \\
$\mathrm{A} 3$ & 8 & 1.510 & $0.0904(14)$ & $1.676(64) \times 10^{-4}$ & $3.91(42)$ \\
$\mathrm{A} 4$ & 8 & 1.510 & $0.0872(22)$ & $1.648(37) \times 10^{-4}$ & $3.95(43)$ \\
\hline $\mathrm{B} 1$ & 12 & 1.475 & $0.1493(5)$ & $1.942(74) \times 10^{-4}$ & $4.32(20)$ \\
$\mathrm{B} 2$ & 12 & 1.500 & $0.1113(8)$ & $1.868(71) \times 10^{-4}$ & $4.337(77)$ \\
$\mathrm{B} 3$ & 12 & 1.510 & $0.09226(92)$ & $1.569(34) \times 10^{-4}$ & $4.61(27)$ \\
\hline $\mathrm{C} 1$ & 16 & 1.475 & $0.1485(4)$ & $2.651(94) \times 10^{-4}$ & $4.503(73)$ \\
$\mathrm{C} 2$ & 16 & 1.490 & $0.1279(2)$ & $2.150(74) \times 10^{-4}$ & $4.612(88)$ \\
$\mathrm{C} 3$ & 16 & 1.510 & $0.09111(31)$ & $1.563(65) \times 10^{-4}$ & $4.90(15)$ \\
$\mathrm{C} 4$ & 16 & 1.510 & $0.09048(52)$ & $1.607(54) \times 10^{-4}$ & $4.90(13)$ \\
$\mathrm{C} 5$ & 16 & 1.514 & $0.08223(34)$ & $1.442(62) \times 10^{-4}$ & $4.98(17)$ \\
$\mathrm{C} 6$ & 16 & 1.519 & $0.06587(37)$ & $1.274(43) \times 10^{-4}$ & $5.23(22)$ \\
$\mathrm{C} 7$ & 16 & 1.523 & $0.04840(54)$ & $1.046(37) \times 10^{-4}$ & $5.61(33)$ \\
\hline $\mathrm{D} 1$ & 24 & 1.510 & $0.09130(27)$ & $1.979(73) \times 10^{-4}$ & $5.061(17)$ \\
$\mathrm{D} 2$ & 24 & 1.523 & $0.04722(43)$ & $1.204(43) \times 10^{-4}$ & $6.058(73)$ \\
\hline
\end{tabular}

Table 5.1: Numerical results for $m_{P C A C}, \chi$ and $\bar{\rho}$ for $N_{\mathrm{f}}=1$ as a function of $m_{\mathrm{PCAC}}$ on lattices of size $2 L \times L^{3}$. Lattice names correspond with those in chapter 4 .

\begin{tabular}{cccccc}
\hline Lattice & $a L$ & $-a m_{0}$ & $a m_{\mathrm{PCAC}}$ & $a^{4} \chi$ & $\bar{\rho} / a$ \\
\hline S0 & 8 & -0.50 & $1.16353(73)$ & $2.198(36) \times 10^{-4}$ & $3.84(34)$ \\
S1 & 8 & -0.25 & $1.07205(97)$ & $2.141(35) \times 10^{-4}$ & $3.85(34)$ \\
S2 & 8 & 0.00 & $0.9706(11)$ & $2.188(37) \times 10^{-4}$ & $3.86(35)$ \\
S3 & 8 & 0.25 & $0.8552(11)$ & $2.059(33) \times 10^{-4}$ & $3.88(37)$ \\
S4 & 8 & 0.50 & $0.7224(13)$ & $1.764(28) \times 10^{-4}$ & $3.96(41)$ \\
S5 & 8 & 0.75 & $0.5607(18)$ & $1.365(23) \times 10^{-4}$ & $4.02(51)$ \\
S6 & 8 & 0.90 & $0.4330(18)$ & $8.23(17) \times 10^{-5}$ & $4.12(67)$ \\
\hline B0 & 12 & 0.95 & $0.39017(68)$ & $4.772(81) \times 10^{-5}$ & $5.73(67)$ \\
B1 & 12 & 1.00 & $0.33623(82)$ & $6.502(93) \times 10^{-5}$ & $6.18(94)$ \\
\hline
\end{tabular}

Table 5.2: Numerical results for $m_{P C A C}, \chi$ and $\bar{\rho}$ for MWT as a function of $m_{\mathrm{PCAC}}$ on lattices of size $2 L \times L^{3}$. Lattice names correspond with those in [10]. 


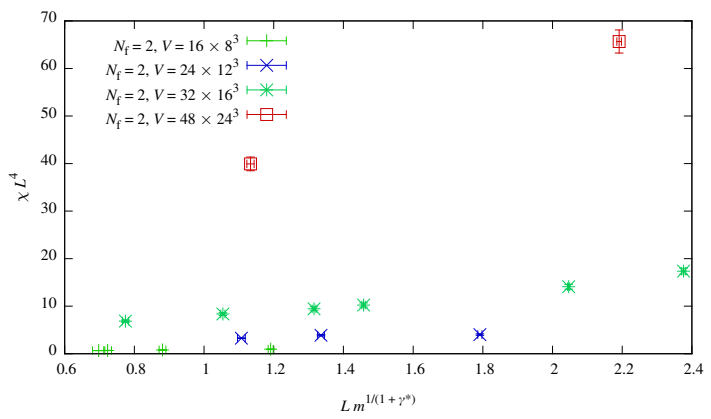

(a) $\gamma_{*}=0.0$

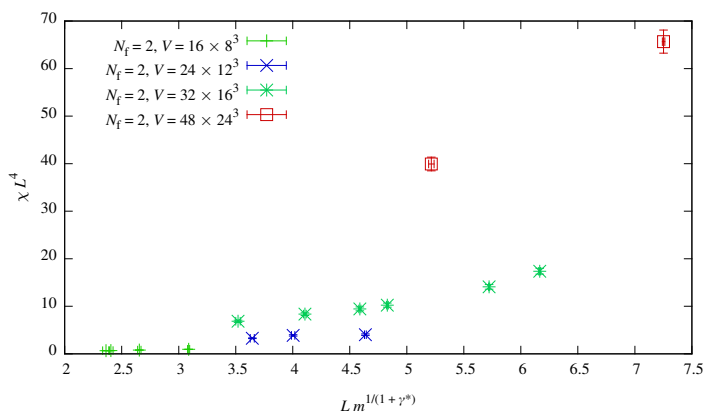

(b) $\gamma_{*}=1.0$

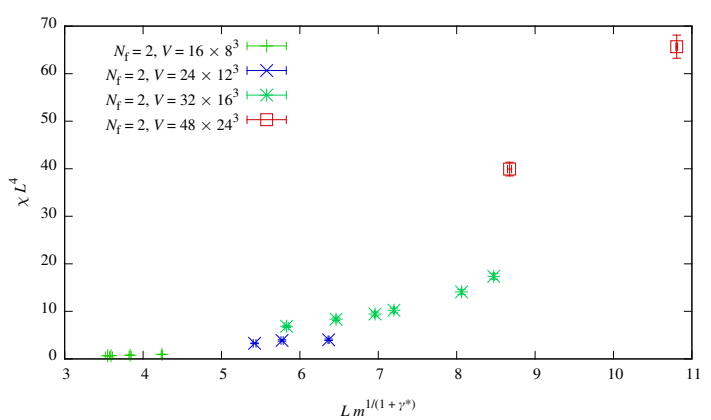

(c) $\gamma_{*}=2.0$

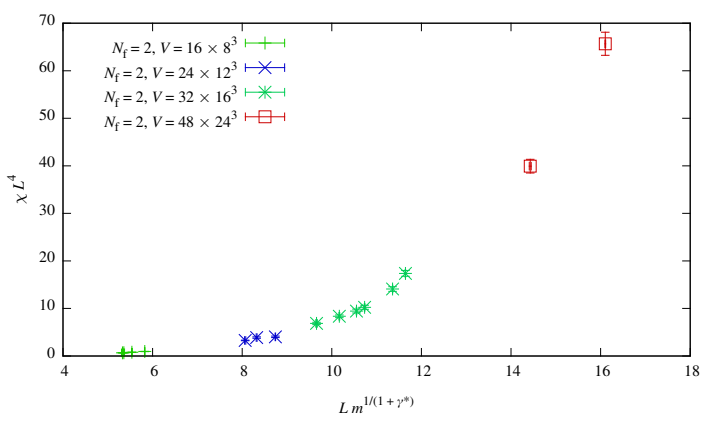

(d) $\gamma_{*}=5.0$

Figure 5.8: Fitting the topological susceptibility for SU(2)Adj Nf1. The closest fit is found at $\gamma_{*} \approx 5.0$, which is well outside the acceptable bounds on $\gamma_{*}, 0 \leq \gamma_{*}<2$. 


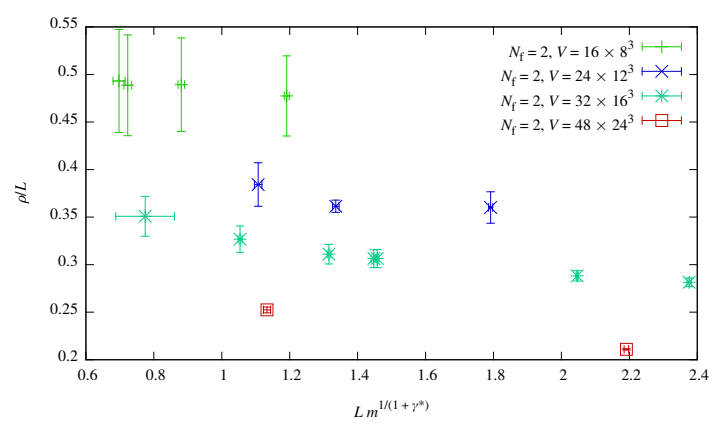

(a) $\gamma_{*}=0.0$

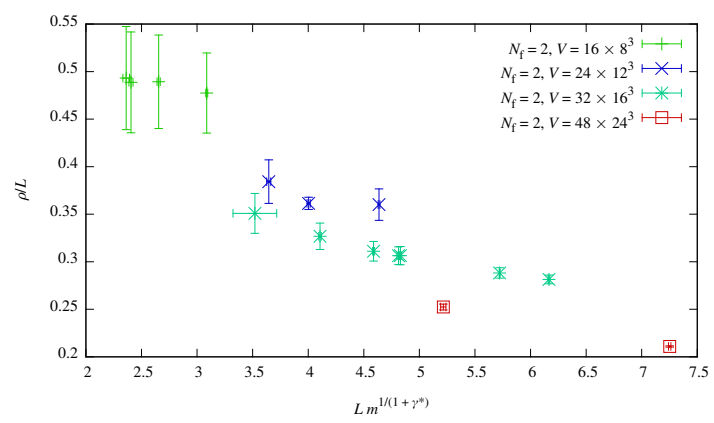

(b) $\gamma_{*}=1.0$

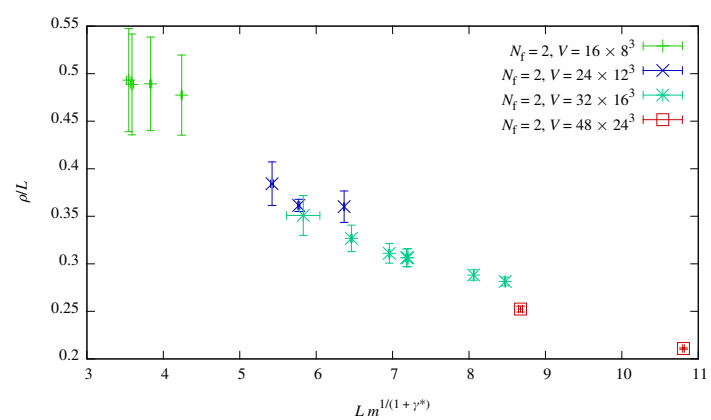

(c) $\gamma_{*}=2.0$

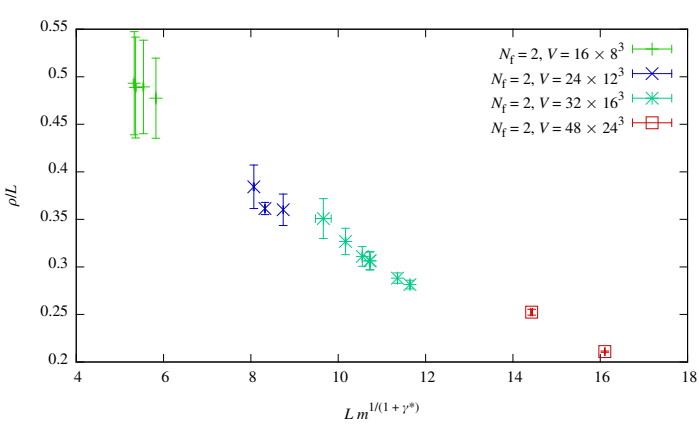

(d) $\gamma_{*}=5.0$

Figure 5.9: Fitting the average instanton size for SU(2)Adj Nf1. Again, the closest fit is found at $\gamma_{*} \approx 5.0$. 


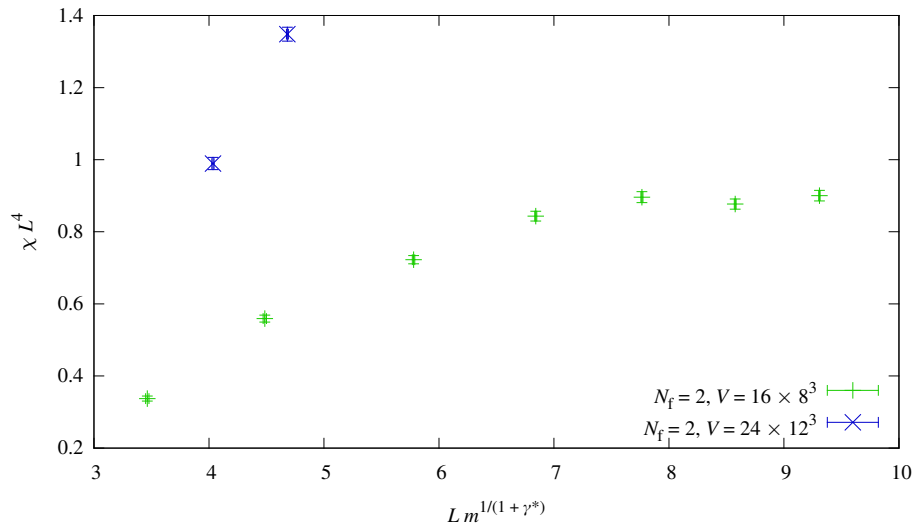

(a) $\gamma_{*}=0.0$

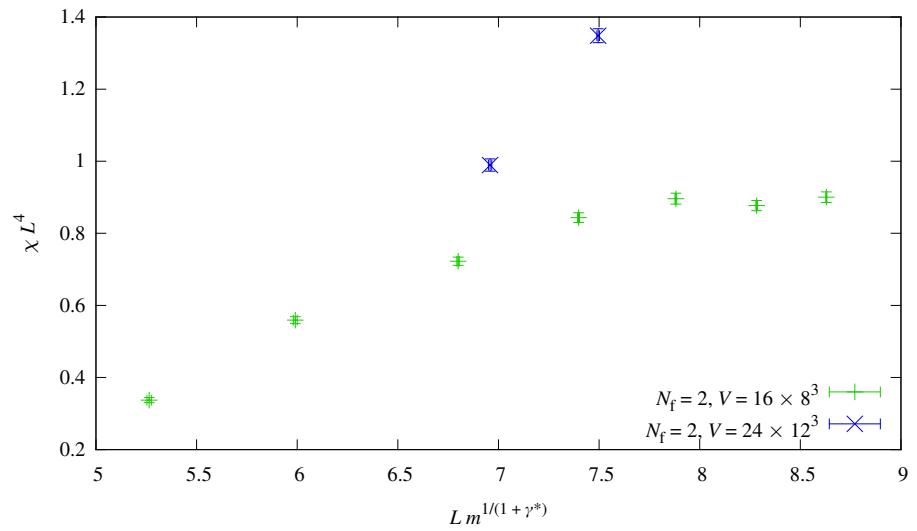

(b) $\gamma_{*}=1.0$

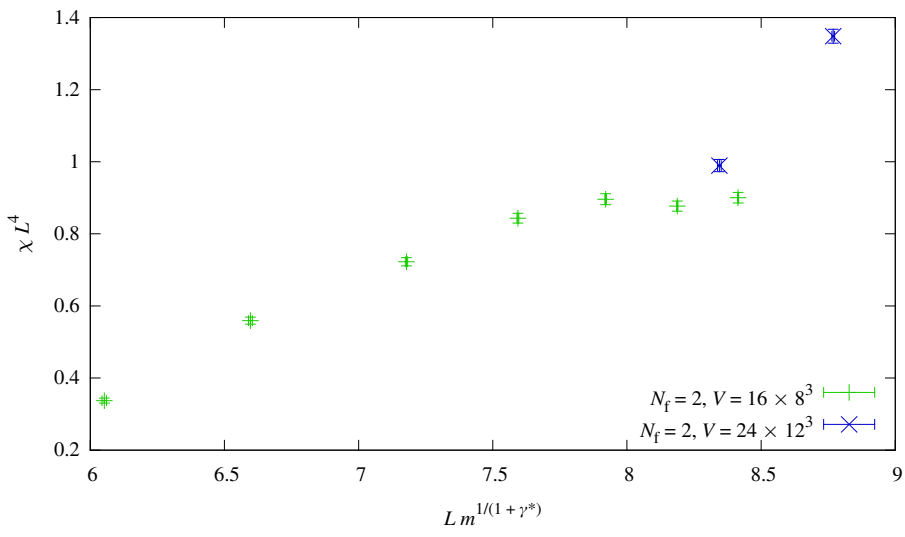

(c) $\gamma_{*}=2.0$

Figure 5.10: Fitting the topological susceptibility for MWT. No good fit is apparent. 


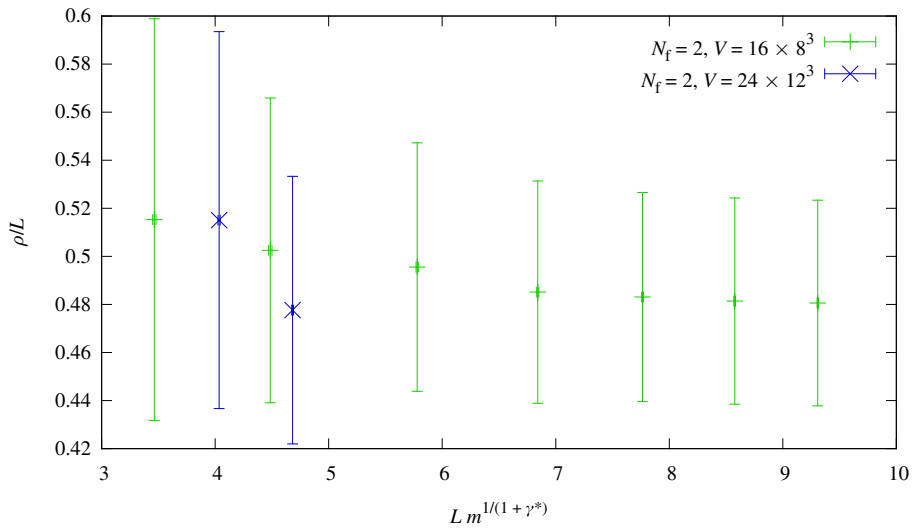

(a) $\gamma_{*}=0.0$

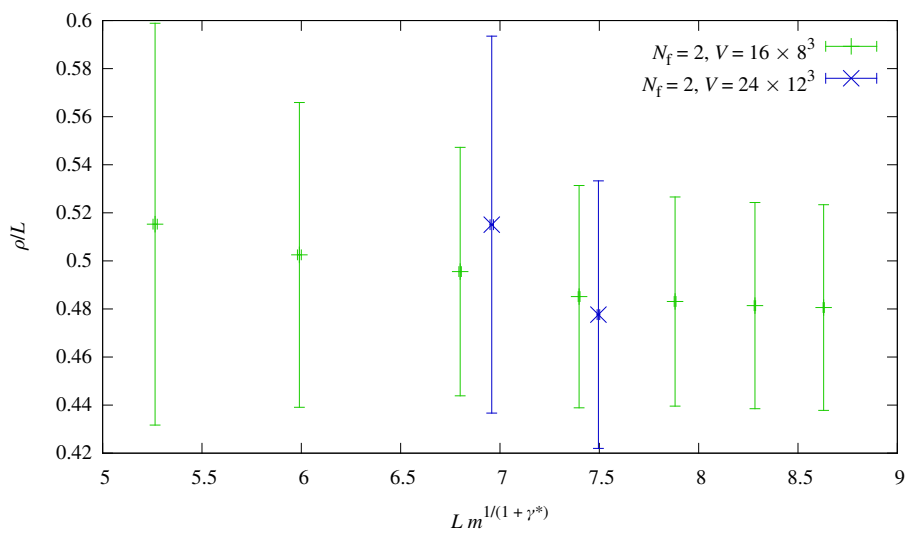

(b) $\gamma_{*}=1.0$

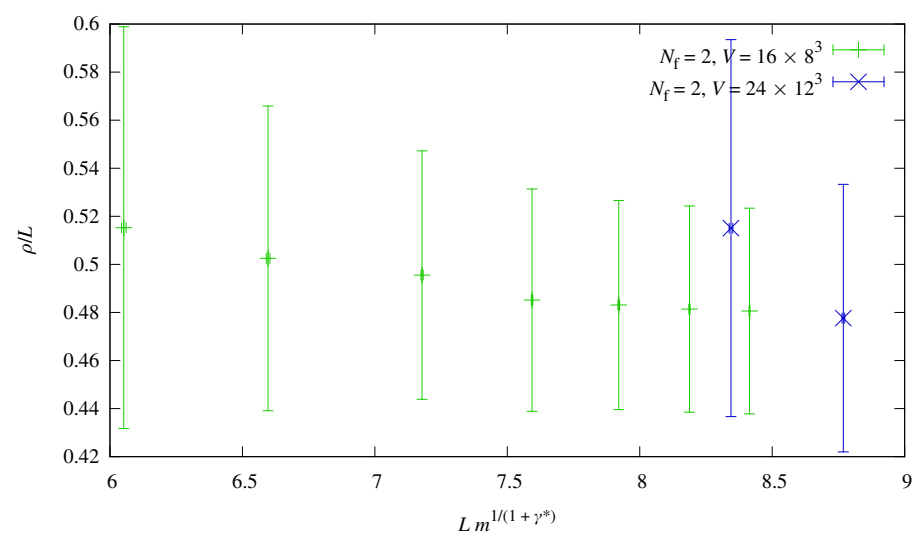

(c) $\gamma_{*}=2.0$

Figure 5.11: Fitting the average instanton size for MWT. All plots fit, since the error bars are larger than the deviation in the data; no bounds may be placed on $\gamma_{*}$. 


\subsection{Discussion}

As was discussed in chapter 2, since MWT is a conformal theory, the fermions are expected to be dynamically quenched, leaving the topological observables unaffected; we therefore expect the MWT and pure gauge results to be equivalent to each other. This is indeed what is seen. Our results from chapter 4 are tentatively indicative of conformality or near-conformality; the consistency seen between the 1-flavour results and the MWT and pure gauge results reinforce these findings.

The distortion of the $20^{4}$ pure gauge distribution is surprising; the values of $\beta$ were chosen to avoid finite-size effects at each lattice volume. It is possible that instantons are more sensitive to finite-volume effects than other objects, but it is more likely that the cooling has caused the smallest instantons to shrink (more so than in the theories with matter) and "fall through" the lattice, distorting the distribution. The SU(2)Adj Nf1 curve at $16 \times 8^{3}$ is a lot closer to the large-volume curve than the MWT curve is; this is most likely because while the MWT study pushed $m$ as low as possible and looked for finite-size effects later, the SU(2)Adj Nf1 study abandoned runs earlier when they showed signs of finite-volume effects, meaning the remaining runs are less afflicted.

For the fits, in the case of $\mathrm{SU}(2) \operatorname{Adj} \mathrm{Nf1}$, the fits only appear reasonable at $\gamma_{*} \approx 5.0$, which is well outside the range of acceptable values for $\gamma_{*}, 0 \leq \gamma_{*}<2.0$. For MWT meanwhile, in the case of $\chi$, no scaling behaviour is observable, and in the case of $\bar{\rho}$, the results are compatible, but their behaviour is such that no bounds can be placed on $\gamma_{*}$.

\subsection{Conclusions}

In this chapter I have presented a first look at the topological susceptibility and instanton size distribution for both SU(2)Adj Nf1 and MWT. Both theories demonstrate behaviour that is compatible with IR conformality in both the instanton size distribution and the topological susceptibility. Both also show finite-size effects in the instanton size distribution at small lattice size, with MWT showing them most severely. Using topological observables, the anomalous dimension of the chiral condensate could not be extracted in either theory.

Extending the MWT study to larger lattices would allow a fuller look at whether or not scaling of topological observables with the anomalous dimension could be observed 
in that theory. It would also be of interest to apply the same method to other candidate walking theories. 


\section{Chapter 6}

\section{HiRep Code and BSMBench}

Having discussed the physical applications of this work in some detail, I will in this chapter present the computational side to the work I have done. First in section $6.1 \mathrm{I}$ will review some of the technical aspects of high-performance computing (HPC). Then in section $6.2 \mathrm{I}$ will outline the HiRep code, which was used to obtain most of the results presented in the previous two chapters. In section 6.3 I will present BSMBench, a new benchmark tool for HPC systems based on the HiRep code.

\subsection{High-Performance Computing}

Before diving into detailed discussions of HPC code and its performance characteristics, it is worth overviewing some of the technical aspects of HPC systems, in order to put the discussion in context.

All modern high-performance (and increasingly non-high-performance) computers operate on the principle of parallelism; that is, running multiple threads of code on distinct processing units. Historically supercomputers were vector processors; that is, they operated on chunks of data in parallel but with scalar instructions. These machines are now mostly confined to museums, ${ }^{1}$ and instruction parallelism is the state of the art. There are two primary methods of execution which are in use, CPUs (Central Processing Units) and Graphics Processing Units (GPUs). CPUs are the older technology and are easier to program for, with each unit being able to execute independently. GPUs, as

\footnotetext{
${ }^{1}$ Note that while machines whose primary method of performance increase is increasing degrees of vectorization are outmoded, the vector execution model is itself present in all modern processors in some respect in the form of SIMD instructions (such as SSE and AVX in x86 processors).
} 


\section{HIREP CODE AND BSMBENCH}

the name suggests, were initially developed to process the display of 3D graphics, but have in the last decade been adapted to run general-purpose code. They offer higher performance than comparably-priced CPUs, but are massively parallel with a limited instruction set, and work in conjunction with a CPU to feed it instructions and process non-parallel sections. Because of this, they are more difficult to program for than CPUs, and since HiRep and BSMBench currently make no use of GPUs, will not be discussed any further.

Worth discussing at this point is the various subdivisions of machines that may be considered. In general large machines consist of a number of nodes (compute cards in Blue Gene parlance), each of which is more or less equivalent to a workstation computer. There is a soft distinction between clusters and superclusters, which use (high-end) commodity processors and have nodes which provide a workstation-like environment, and "true" supercomputers (most notably IBM's Blue Gene architecture ${ }^{1}$ ) which use specialist CPUs and have stripped-down nodes. (Historically the word "supercomputer" was reserved for machines with huge amounts of memory shared between a large number of execution cores and/or vector units, and even a Blue Gene-like machine would be referred to as a supercluster; this nomenclature is now limited to a handful of traditionalists, since such machines have been largely discontinued in favour of the simpler node-based parallelism.) For code that is not parallelised, clusters generally offer better performance for job-level parallelism (sometimes referred to as High-Throughput Computing (HTC), running multiple scalar codes simultaneously to take advantage of the machine's parallelism); while this is sometimes possible on Blue Gene-like machines, it is generally less efficient than using a cluster environment.

Each execution core is effectively a CPU in its own right, having dedicated caches, and being able to execute instructions independently of its siblings; the cores frequently also share access to a common cache. For the purposes of running code, the divisions between processors are not normally considered, and the node is considered in terms of its number of cores only. All the cores on a node share access to the same memory,

\footnotetext{
${ }^{1}$ For completeness, the Blue Gene architecture also has higher-level structure above the node level: the compute cards are arranged into blocks of 16 called node cards, which are in turn arranged into midplanes. Two midplanes placed one atop the other (possibly with some additional I/O hardware) form a rack, which is the smallest unit of Blue Gene available for purchase. The machine then comprises an appropriate number of racks.
} 


\subsection{High-Performance Computing}

thus the node (as with the entire historical supercomputers discussed above) is a sharedmemory environment.

Since the memory is not shared between the nodes (rather being distributed between them), is is almost always the case that the contents of memory will need to be transferred between nodes (since it is rare that a parallelised code will only work with the portion of the data set that it is allocated, and not depend on any updated data from other nodes). Because of this, it is important to have high-speed interconnects between the nodes. Lattice codes in particular, even with ultralocal updates, need to synchronise large amounts of data between nodes very often; it is therefore important that the interconnects have both high speed and low latency. Speed here refers to the amount of data transferred in a given time, while latency refers to the minimum delay between making a request and receiving the resulting data.

To illustrate the difference, a frequently-quoted example of a high-speed, highlatency connection is a high-speed train loaded with flash memory - the train moves slowly compared to electrical signals, but the data density makes the overall transfer rate better than electrical signals. (A numerical discussion of this type of scenario may be found in, for example, [176].) Meanwhile a Morse code operator on a radio frequency would have a lower throughput speed (limited by the human finger), but much lower latency (the speed of light providing the lowest possible latency). A high latency would leave a lattice code frequently waiting for a transfer to commence so it can continue, while a slow speed would leave it waiting for the transfer to complete.

An execution core may also support multiple concurrent threads - in Intel chips this is referred to as HyperThreading. This was originally introduced as a "poor person's multi-core", since for commodity usage it provides advantages similar to a multi-core chip on a single-core processor. The processor contains multiple sets of state registers (or caches for them); however, only one thread executes at a time. The speed improvement comes from the vastly increased speed of context switching, for which efficient instructions are added. This means that when a program needs to wait (for network traffic, for example), the CPU may switch to a different process that is ready to continue, without the "thrashing" that occurs in the absence of concurrent threads.

There are two primary methods of parallelising applications: message-passing methods such as MPI (Message Passing Interface), and thread-based shared-memory approaches such as OpenMP and Pthreads. In the case of MPI, $N$ copies of the same 


\section{HIREP CODE AND BSMBENCH}

executable are run, where $N$ is the degree of parallelisation. It allows parallelisation both on single nodes and across multiple nodes, since the messages can be passed locally or over a network; however, the cost incurred is duplication of data between the processes, and some message-passing overhead when running in a shared-memory environment. Multithreaded approaches meanwhile run only one executable, spawning threads sharing the same execution space to share the work; this avoids the overheads associated with MPI, but are limited to running on a single node since multiple nodes do not (in general) share memory.

It is possible to combine these approaches into "hybrid" MPI-OpenMP programs, with multithreading on the node and message passing between the nodes; this avoids as many message-passing overheads as possible, but two sets of parallelisation requires twice as much debugging, and care must be taken to ensure that the two don't interfere with each other. Some compilers will attempt to automatically implement multithreading optimisations; however, with current non-trivial code, there is not a measurable improvement in performance from these optimisations.

\subsection{HiRep}

The HiRep code has been written over the last six years by a team headed by Claudio Pica. Much work has been published incorporating results produced by HiRep and code built upon it $[10,14,156,157,177-187]$. Its operation is described in detail by Del Debbio, Patella, and Pica [178]; the algorithms used are reviewed in chapter 3.

The HiRep code comprises:

- A set of C modules containing shared functions

- A set of $\mathrm{C}$ and Fortran programs for specific computations, making use of the above libraries

- $\mathrm{C}++$ code to generate header files for a particular fermion representation and gauge group at compile time

- Makefiles to link the above together

- Additional statistical analysis tools $(\mathrm{C}++$ code with bash wrappers) to extract spectroscopic quantities from the program output 


\subsection{BSMBench}

Compile-time parameters (e.g. gauge group, fermion representation, boundary conditions, use of MPI) are specified in a flags file. Runtime parameters (e.g. lattice volume and parallelisation, coupling and bare mass, directory for writing configurations, algorithmic parameters) are set in an input file, which takes the form of key = value pairs, one per line. A few parameters (e.g. run log file location) are set as command-line arguments.

As is common for lattice codes, parallelisation occurs using MPI, with the division splitting the lattice volume into chunks, one per process. In the absence of compilerspecific automatic multithreading optimisations, each MPI process is single-threaded.

\subsection{BSMBench}

BSMBench is a HPC benchmark tool developed from the HiRep code. In this section I will explain the need for benchmarks in HPC, outline the procedure adopted for BSMBench, and then present some results the benchmark gives on various machines, with accompanying discussion. An earlier version of this work was presented as a poster [15] at the International Supercomputing Conference (ISC'12) [188] in Hamburg.

\subsubsection{Why benchmark?}

Every application written for a system makes different demands on its hardware. For example, one application may deal exclusively with integers, while another makes heavy use of floating-point numbers. So-called "big data" applications, which are becoming of increasing importance in HPC with the rise of large datasets from social networking applications, require vast amounts of working memory accessible to a single process; other applications (including most lattice gauge theory computations) may be parallelised such that on-node memory is not a major constraint.

This diversity of applications means that it is impossible for a supplier of a machine to quote a single performance rating that fits all applications. This has led to the creation and standardisation of benchmarks. A benchmark is a reference program, having the same characteristics as a production program in a particular area (ideally from the same codebase), which behaves in a predictable way; when run on an ensemble of machines, the output may then be used to compare the machine's suitability for that task. Having this measure is invaluable when making procurement decisions for dedicated 


\section{HIREP CODE AND BSMBENCH}

facilities: if a machine with characteristics designed to suit a graph application were bought to run physics applications, for example, performance would be far lower than would be had from a similarly-priced machine that was better-suited to the task.

The LINPACK benchmark [189] is the most commonly-cited benchmark for HPC, and is the basis of the TOP500 [190] ranking; there is, however, increasing debate over its continued relevance ([191], for instance, suggests the LINPACK benchmark as one of a suite stressing different areas, and [192] warns of the danger in designing new large machines to run LINPACK when they will be used for other purposes). The Graph 500 [193] list in 2010 introduced a new benchmark for Big Data-style applications; this has illustrated the disparity in demands, with the rankings of machines shuffling greatly compared to their TOP500 places. QCD has been used to both benchmark supercomputers [194] and smaller machines [195]; however, these benchmarks have not produced ranking tables in the same vein as the TOP500 and Graph 500.

The highest-performance machines available are generally the newest to market and most bespoke, and thus do not benefit from the accumulated years of testing an older or more commonly-used system will have. This means that when running a benchmark on a new machine, the test should include a consistency check to verify that the code is functioning correctly. Failing to do this could result in procuring a machine that appears blisteringly fast compared to the competition, only to find that it fails to run the main body of the code. BSM code in general and HiRep in particular, unlike most code, has the flexibility to adjust its ratio of communications to compute demands, based on the fermion representation and the gauge group. This allows a benchmark derived from it to give results applicable to more applications than existing benchmarks.

\subsubsection{Benchmark strategy}

To summarise the relevant points from the previous subsection, we have two main aims for the benchmark code [196]:

1. To give a characteristic rating of the machine's performance, and

2. To check that the machine executes the code correctly.

The benchmark should fulfil these two tasks within certain constants: 
- It should use code that is representative of the main body of the computation being benchmarked.

- It should give output that can be read by people who are not knowledgeable in the area of the benchmark, so that system builders can run the benchmark internally and understand its output.

- It should be sufficiently large that the machine in question can demonstrate its power (i.e. not so short that initialisation times dominate, or so small that only a small portion of a machine can be used)

- It should run in reasonable time (30 minutes - 1 hour is ideal, although due to its age and design LINPACK can take over 24 hours to run on the largest current machines; there is active research into how to reduce this time whilst still maintaining a comparable output)

- It should be reliable and repeatable (if one machine is rated as having better performance than another, it should always do so for that metric, and repeated runnings of a test should give the same score)

Performance is often measured in FLOP/s (Floating-Point OPerations per second) for numerical applications; obviously for other applications this would be a less relevant rating. When a benchmark is being used for a specific application rather than in an attempt to give a general overall picture of a machine's speed, any unit that can be consistently compared could be used - for example, "time to complete one Monte Carlo update", or "number of Dirac operator applications in ten minutes" would be suitable measures for a lattice gauge theory benchmark.

As was mentioned in the previous subsection, whilst QCD has already been used as a benchmark for machines being considered for QCD applications, looking at BSM theories changes the ratio of compute to communications demands - which in the case of QCD are roughly balanced. We therefore need a benchmark to look at the classes of BSM theories we are interested in using a machine for; a QCD benchmark would be insufficient, since a deficiency in the area necessary for the BSM theory could be masked by a good performance in another area. Additionally, developing such a benchmark will allow application to areas outside of lattice physics, since the flexibility in the choice of theory allows altering the demands of the benchmark. 


\section{HIREP CODE AND BSMBENCH}

We choose therefore to make three different regimes available to benchmark:

- A communications-intensive regime, $\mathrm{SU}(2)$ with two adjoint flavours, referred to as the "comms" test,

- A balanced regime, $\mathrm{SU}(3)$ with two fundamental flavours, referred to as the "balance" test, and

- A compute-intensive regime, $\mathrm{SU}(6)$ with two fundamental flavours, referred to as the "compute" test.

More tests can be easily added by anyone with access to the HiRep code.

The strategy adopted for the benchmark is adapted from that of Lüscher [195]. The program's preparation and execution occur as follows:

- Preparation:

- The user prepares or selects a machine configuration file specifying their C compiler and appropriate flags; some such files are included with the distribution for machines with which BSMBench has been tested.

- (Optional) The user may set a flag to indicate that they do not require a parallel version of the code.

- The user calls the compile script with the parallel (and optionally the nonparallel) machine configuration file as argument(s).

- The compilation script copies the machine configuration file into the appropriate place and sets appropriate flags in the HiRep configuration files.

- The compilation script then calls the make command to generate the three versions of the benchmark listed above, in parallel (unless told otherwise) and (optionally) non-parallel versions.

- Execution:

- The user (or job control system) calls (via mpirun if appropriate) the appropriate benchmark executable, pointing it at one of the supplied parameter sets

- The code reads in the parameter set, defining the geometry appropriately. 


\subsection{BSMBench}

- The code allocates a random gauge field and as many random fermion fields as are specified in the parameter set.

- As a consistency check (item 2 above), the code inverts the Dirac operator on the first random spinor field and ensures that the residual drops below a given threshold. The input and output spinors are then re-randomised. This test is only carried out if requested in the parameter set, since it would dominate the execution time on smaller machines (e.g. workstations), where it will generally be unnecessary. The total number of iterations and overall time taken is recorded.

- The code calculates the square norm of each random spinor field in turn a given number of times, repeating until a time threshold is passed. The number of iterations starts at one, and doubles each time the process repeats, to avoid spending excessive time checking the progress.

- The code performs the operation $\psi_{2}=\psi_{2}+c \psi_{1}$, where $c$ is a complex scalar constant, on successive pairs of random spinor fields, repeating in the same manner as above.

- The code performs a Dirac operator application $\psi_{2}=D \psi_{1}$ on successive pairs of random spinor fields, repeating in the same manner as above.

- The code uses its results and reference measurements from a Blue Gene/P to calculate the FLOP/s rating, and output absolute performance information as well as a relative comparison to a reference Blue Gene/P machine.

For the purposes of comparing machines' performance, the Dirac operator application test is the most revealing.

The structure of the code is as follows. In the source distribution, there is:

- A directory containing a stripped-down HiRep distribution, with the addition of the benchmark code and comments on the licensing terms,

- A directory containing sample machine configuration files,

- A directory containing parameter sets for the default set of tests,

- An empty directory to hold output files, 


\section{HIREP CODE AND BSMBENCH}

- The README and LICENSE text files (see appendix B), and

- The compilation script.

The supplied tests work with a lattice size of $64 \times 32^{3}$, to allow for comparison from workstation-class machines up to small supercomputers. Comparing results of tests at different lattice sizes is not recommended, since the associated overheads change. Additionally, the maximum parallelisation recommended is that giving a local lattice of $8 \times 4^{3}$; further parallelisation unreasonably increases the overheads. This limits the stock tests to 4096 MPI processes; equivalent to a single Blue Gene/P rack in Virtual Node mode. This limit could be removed by choosing a larger lattice size, as is done later in this section for the extended tests of Blue Gene/Q; however, this would make running the tests impractical on workstations. 4096 MPI processes is sufficient as a proof-of-concept and a test of moderately-sized machines.

\subsubsection{Tests performed}

The code has been used to systematically test a number of machines:

- Two clusters run by HPC Wales, one in Cardiff and one in Swansea,

- The BlueIce2 cluster in Swansea,

- An IBM Blue Gene/P machine (which was used as the reference configuration); runs were split between the UKQCD machine in Swansea and a machine operated by IBM Research in Yorktown Heights, New York,

- An IBM Blue Gene/Q machine, also at IBM Research in Yorktown Heights,

- The ULGQCD cluster in Liverpool, and

- A Mac Pro in Swansea.

Relevant details of the machines are listed in table 6.1.

Most queue managers ignore any concurrent thread support of the CPUs, since MPI rarely gains an advantage by running multiple concurrent threads; multithreaded code must be written or compiled in to use this ability. Blue Gene/Q, however, does provide for this, thus up to 64 MPI processes may be run on a single processor. 


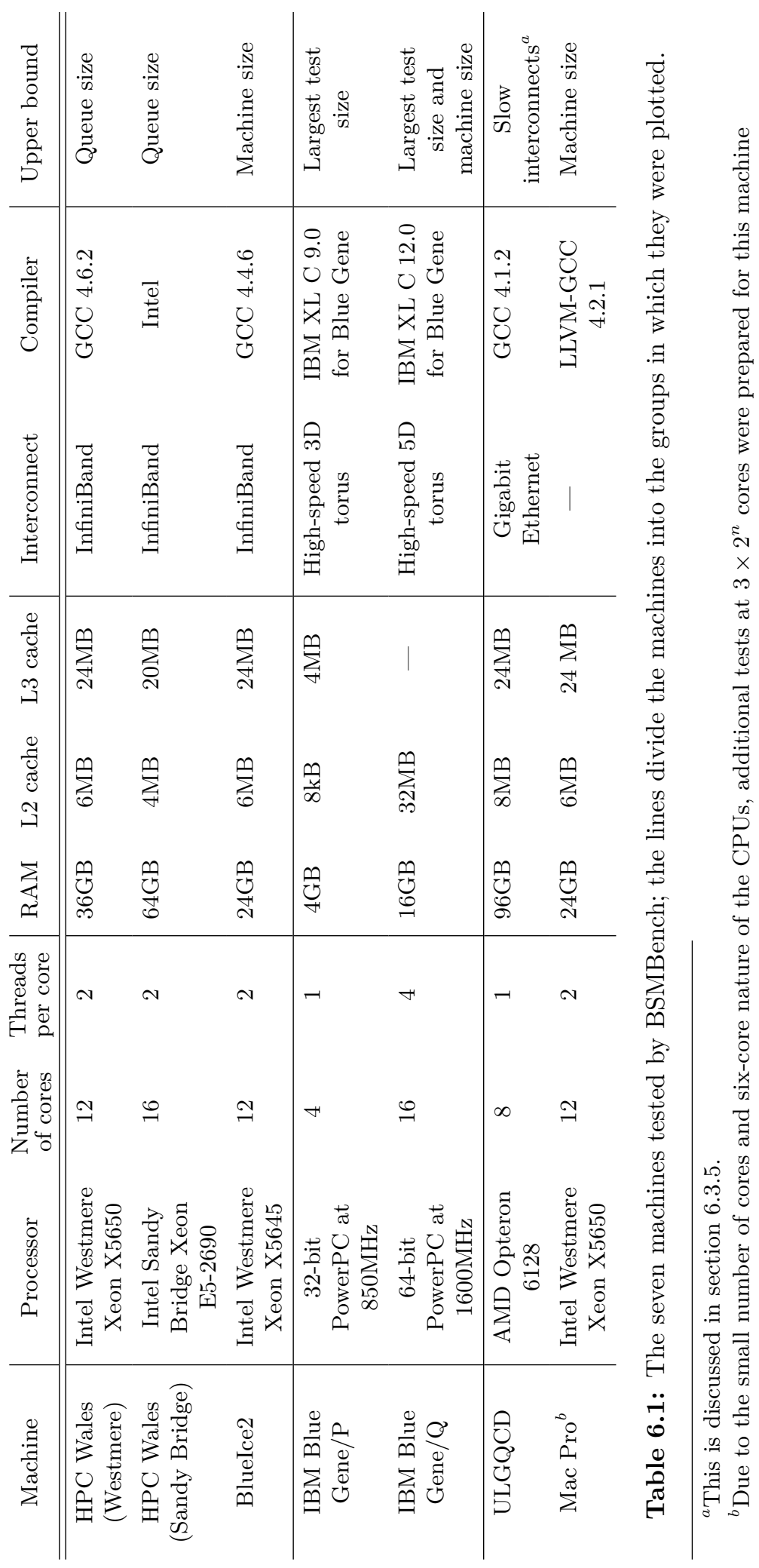




\section{HIREP CODE AND BSMBENCH}

The primary test performed was running each of the benchmark tests on each machine at increasing partition sizes, from the minimum on which the tests would run (with node memory being the primary constraint) up to an upper limit determined by the factors listed in table 6.1. All tests were run with one MPI process per processor core - sometimes referred to as Virtual Node (VN) mode - since HiRep and BSMBench are not currently multithreaded.

Two other tests were run: firstly, a customised set of tests with a $128 \times 64^{3}$ lattice on Blue Gene/Q to allow a rack-for-rack comparison with a Blue Gene/P; this was again run in VN mode. Finally, a set of tests were run on a single Blue Gene/Q partition, varying the number of MPI processes per node from 1 to 64 , to test the performance of the multiple hardware threads of the Blue Gene/Q processor.

\subsubsection{Results}

The plots are divided into groups by machine class to avoid overcrowding them. The primary battery of tests, shown in figures $6.1-6.8$ shows a roughly linear relationship between FLOP/s performance and number of MPI processes (i.e. processor cores in use) in all cases. All machines show a slow drop in performance per node as the number of nodes is increased, associated with the increased overheads. The ULGQCD cluster shows a rapid tail-off in performance once the parallelisation exceeds the size of one node; for the largest size tested not only does the per-core performance drop, but the overall performance of the partition drops.

The rack-for-rack comparison, shown in figure 6.9 shows that for larger lattices Blue Gene/Q continues to scale as we expect. The comparison of different processor subdivisions, shown in figure 6.10 shows linear scaling up to 1 process per core. When further subdividing the cores, the performance plateaus, showing little to no gain to using more than one thread per core.

\subsubsection{Discussion}

It is worth drawing attention to some features of the data that are particularly interesting. For this discussion I will concentrate on the Dphi test, since it is most representative of "real-world" performance.

For the Blue Gene/P, unexpected upticks in performance were seen at 512 and 4096 cores (128 and 1024 nodes, or $\frac{1}{4}$ and 1 rack respectively) that were not seen on the Blue 


\subsection{BSMBench}

Key to symbols:

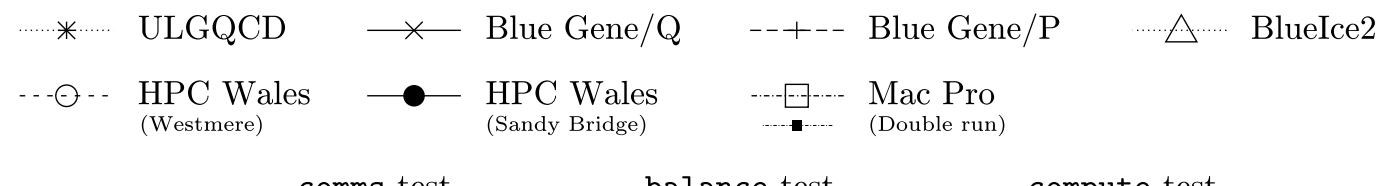

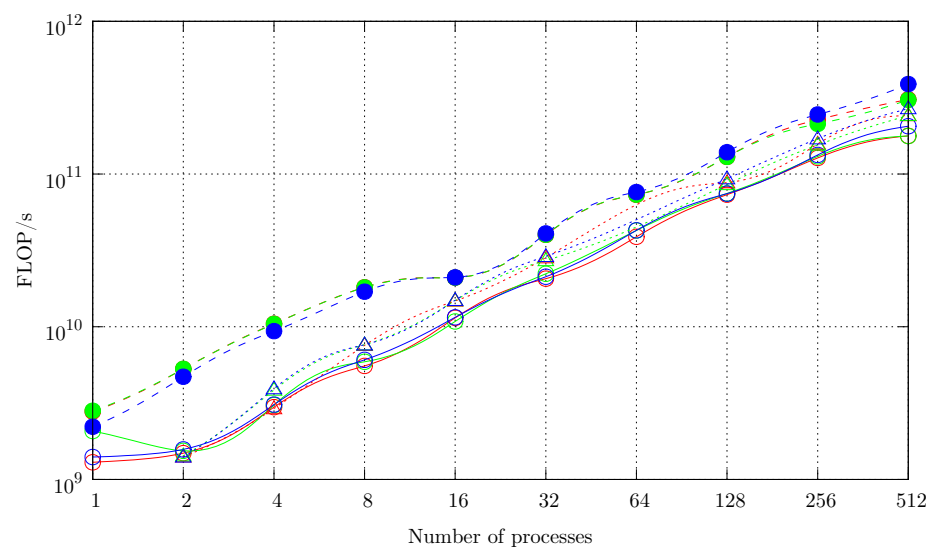

(a) HPC Wales and BlueIce2

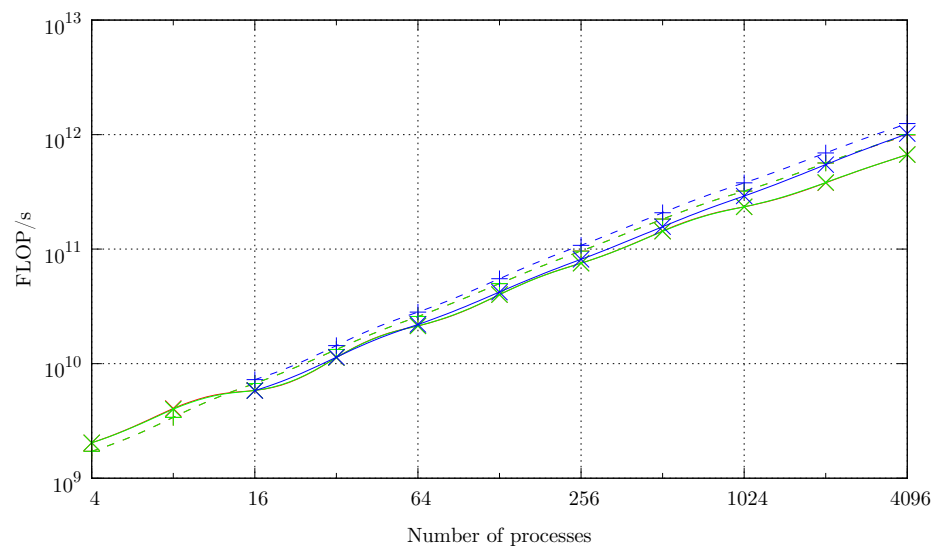

(b) Blue Gene

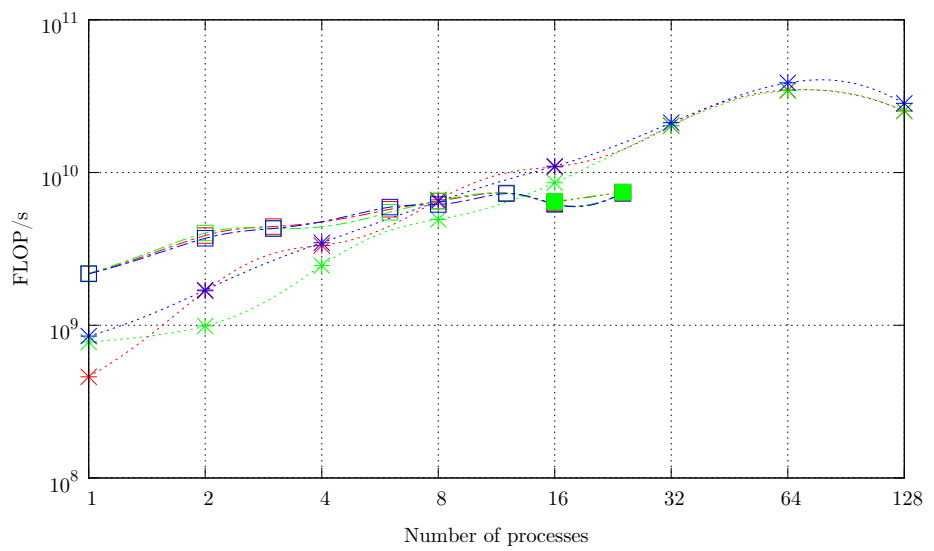

(c) Others

Figure 6.1: Results of the spinor field square norm test plotted for the whole ensemble. 


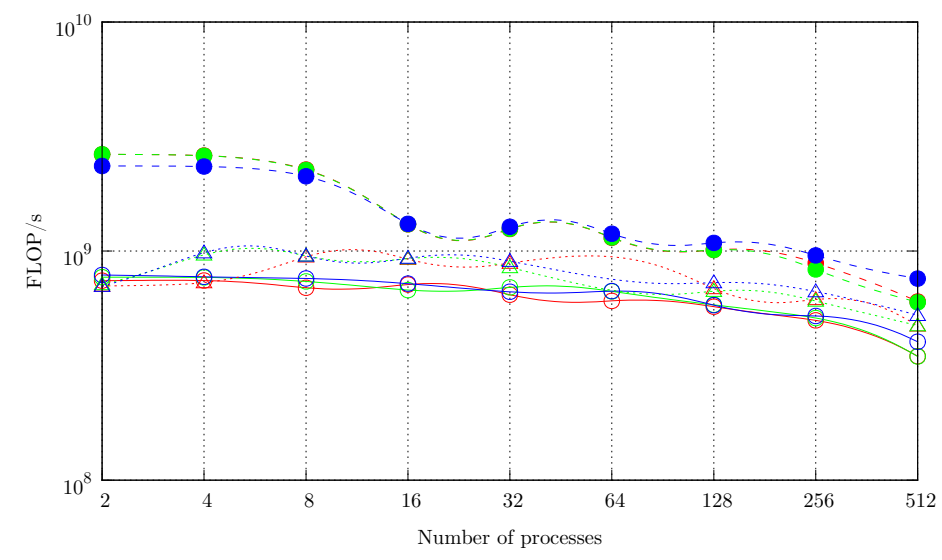

(a) HPC Wales and BlueIce2

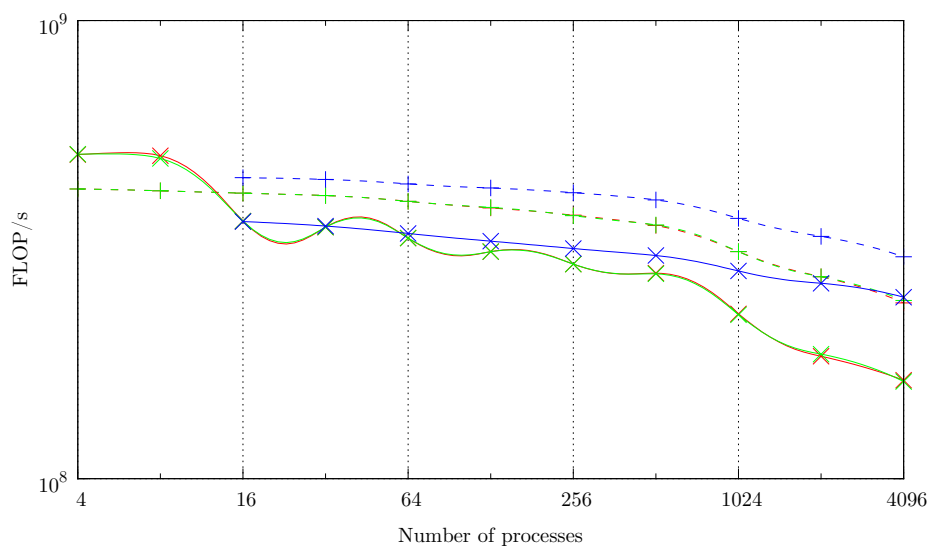

(b) Blue Gene

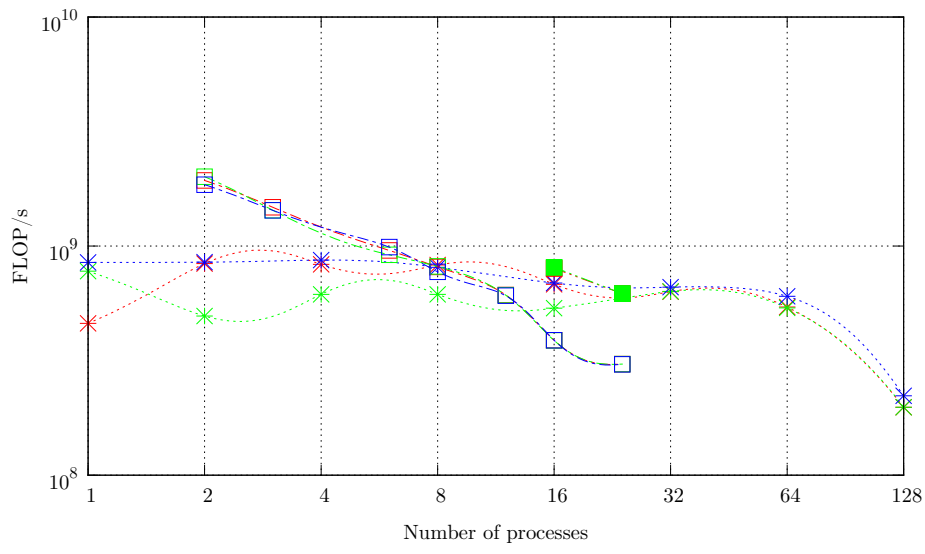

(c) Others

Figure 6.2: Results of the spinor field square norm test, plotting the average performance per MPI process. 


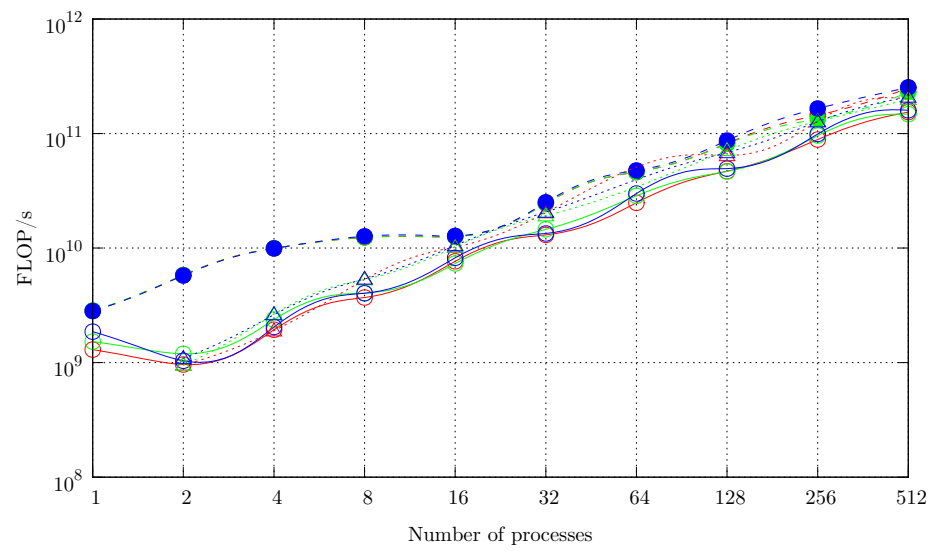

(a) HPC Wales and BlueIce2

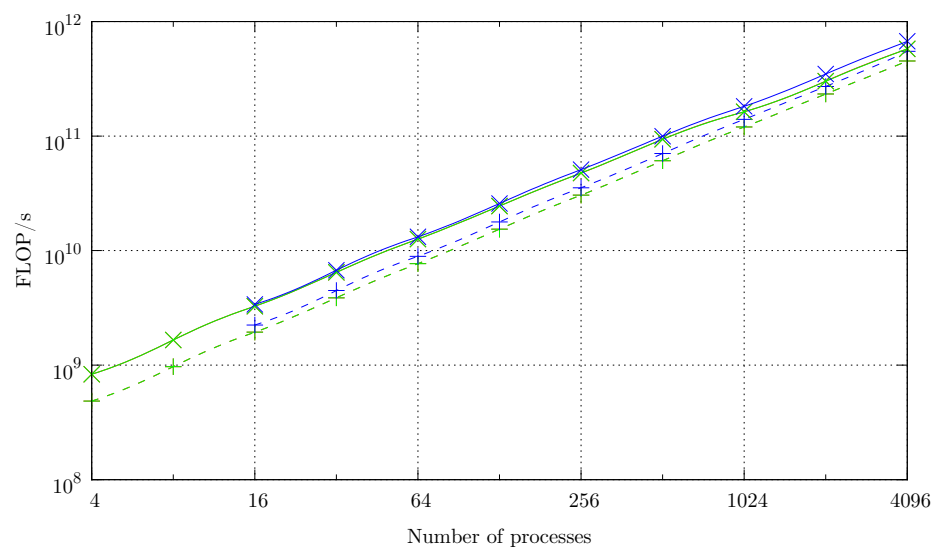

(b) Blue Gene

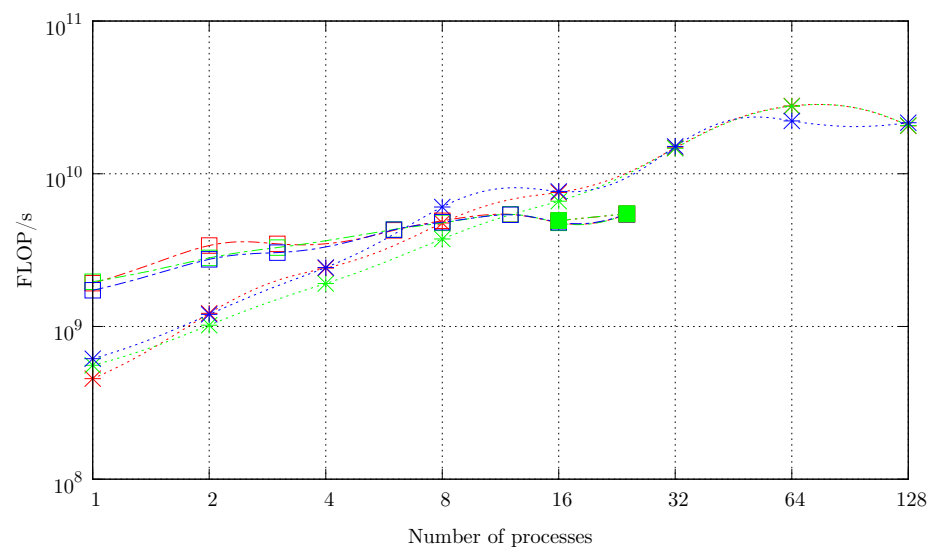

(c) Others

Figure 6.3: Results of the spinor field multiply-add, plotted for the whole ensemble. Symbols as on page 105 . 


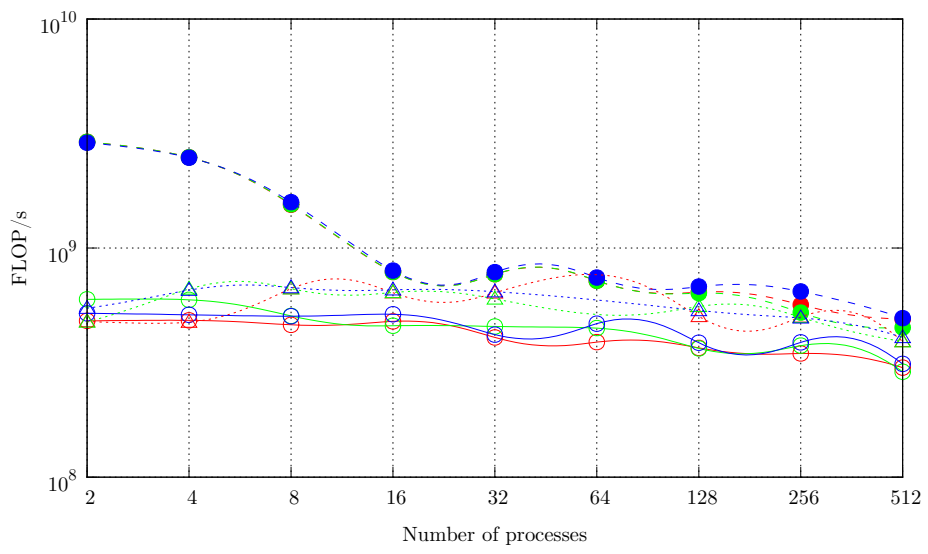

(a) HPC Wales and BlueIce2

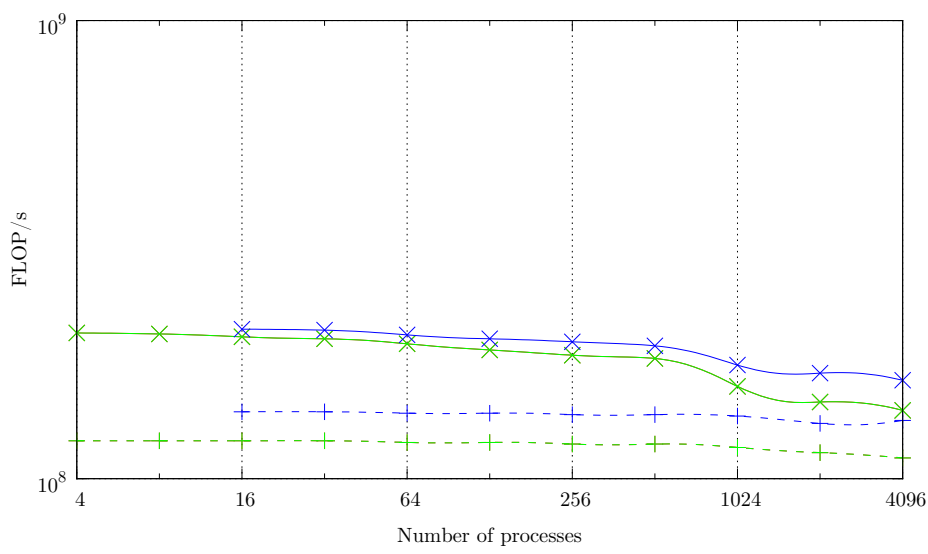

(b) Blue Gene

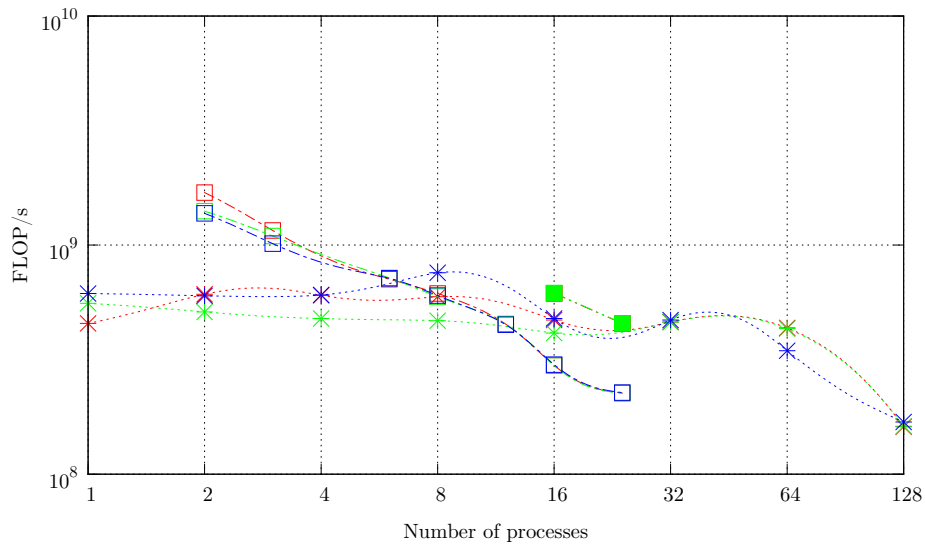

(c) Others

Figure 6.4: Results of the spinor field multiply-add, plotting the average performance per MPI process. Symbols as on page 105. 


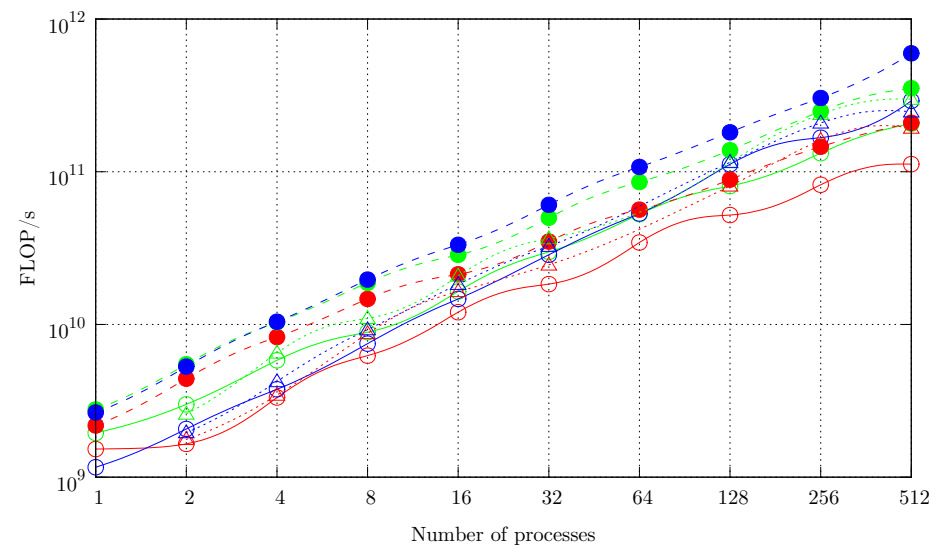

(a) HPC Wales and BlueIce2

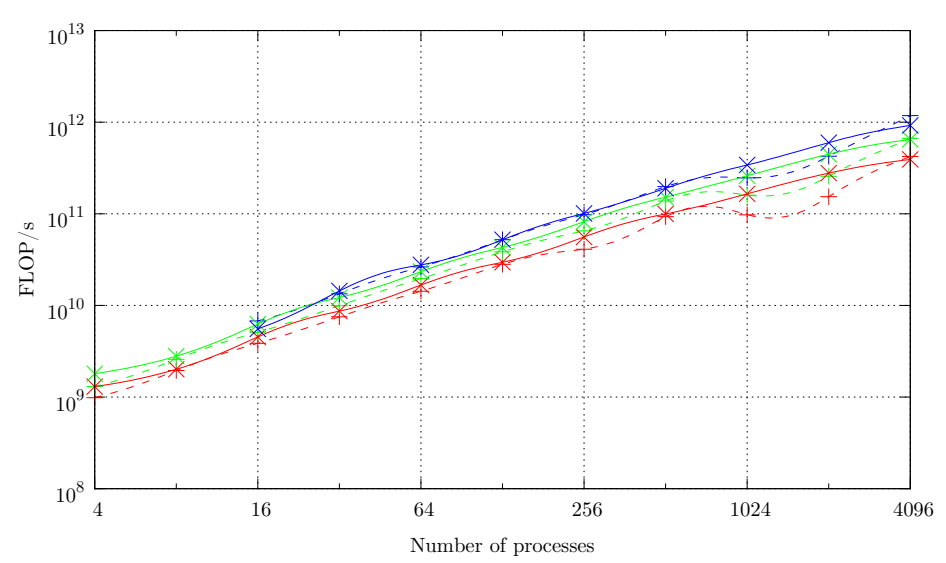

(b) Blue Gene

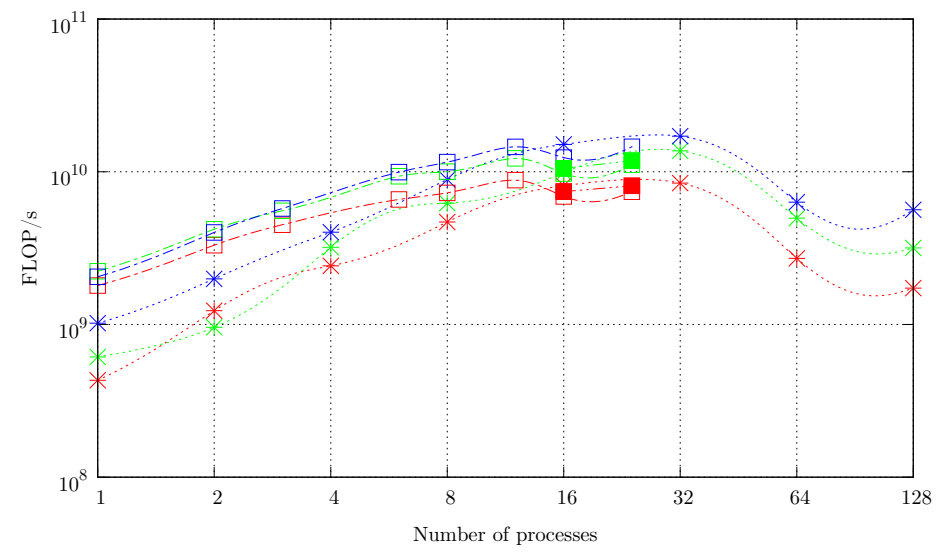

(c) Others

Figure 6.5: Results of the Dirac operator application test, plotted for the whole ensemble. Symbols as on page 105 . 


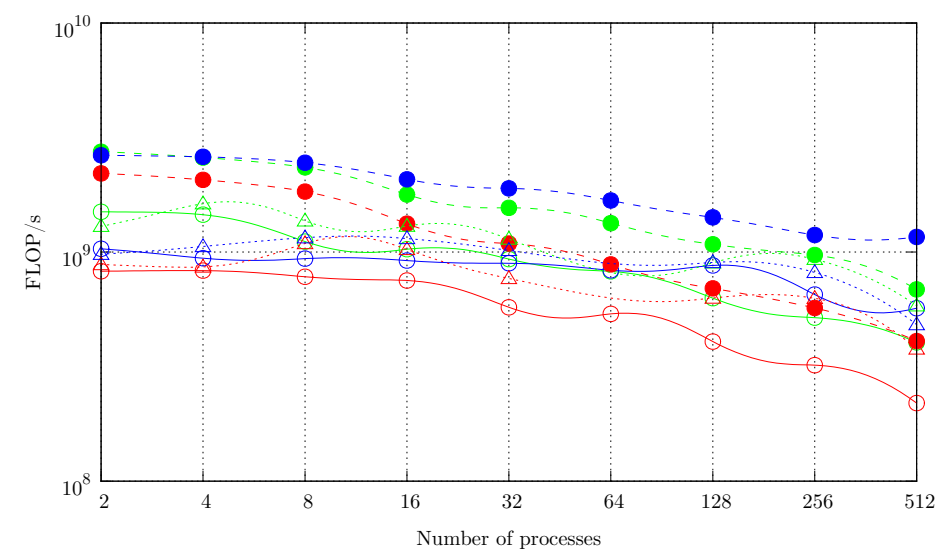

(a) HPC Wales and BlueIce2

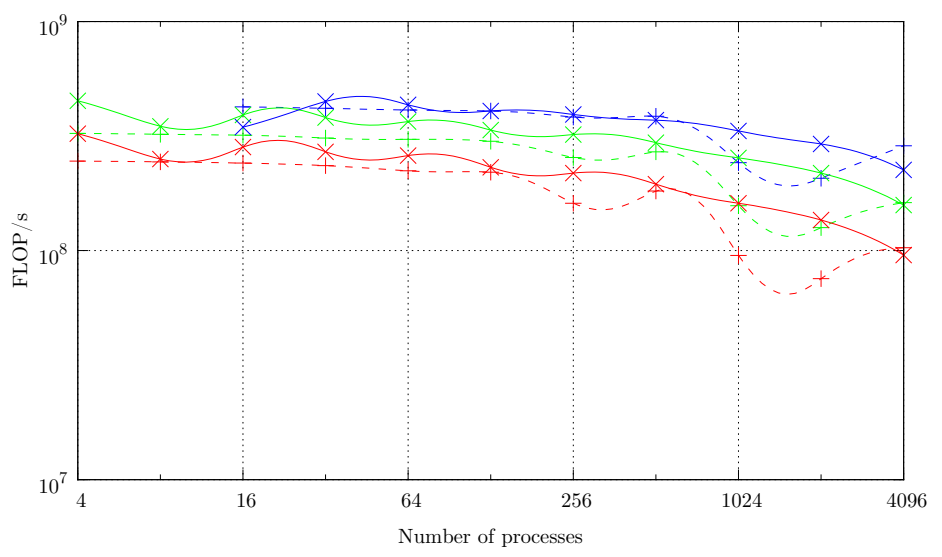

(b) Blue Gene

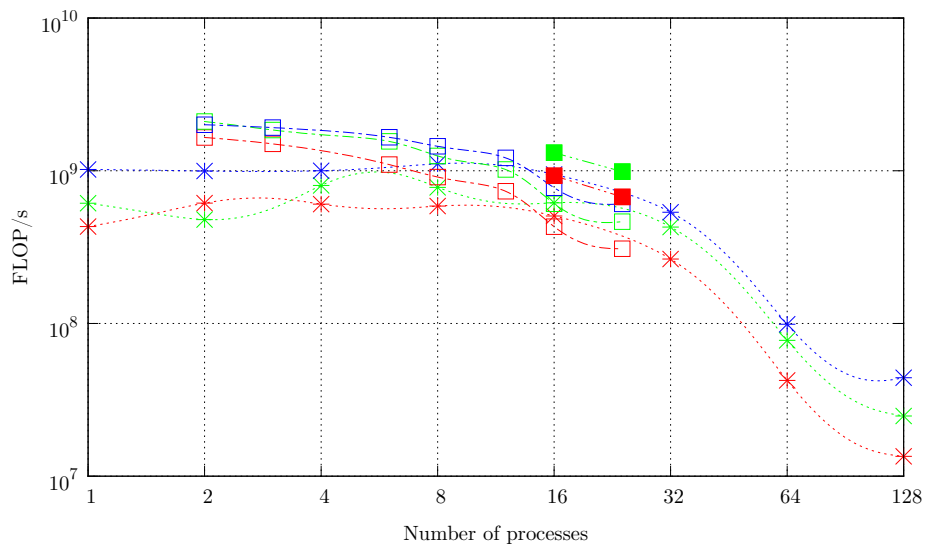

(c) Others

Figure 6.6: Results of the Dirac operator application test, plotting the average performance per MPI process. Symbols as on page 105. 


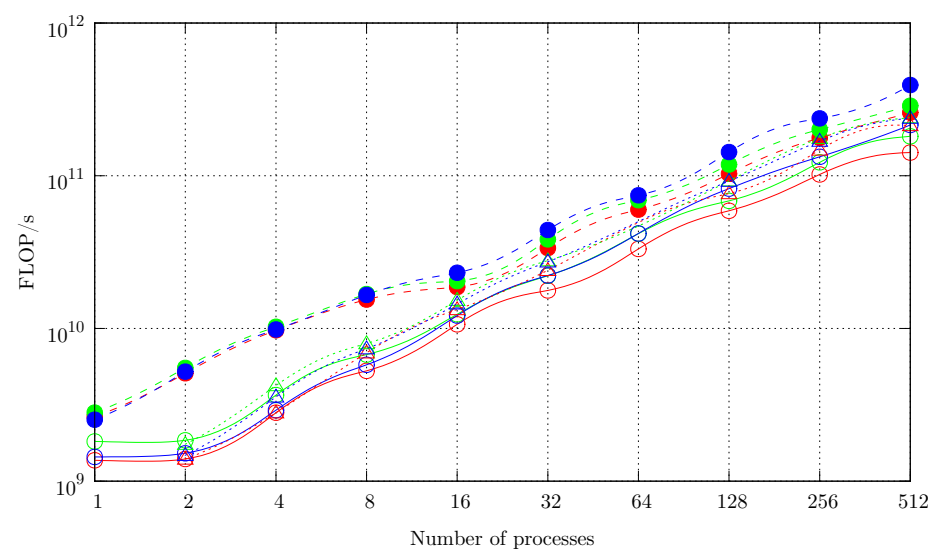

(a) HPC Wales and BlueIce2

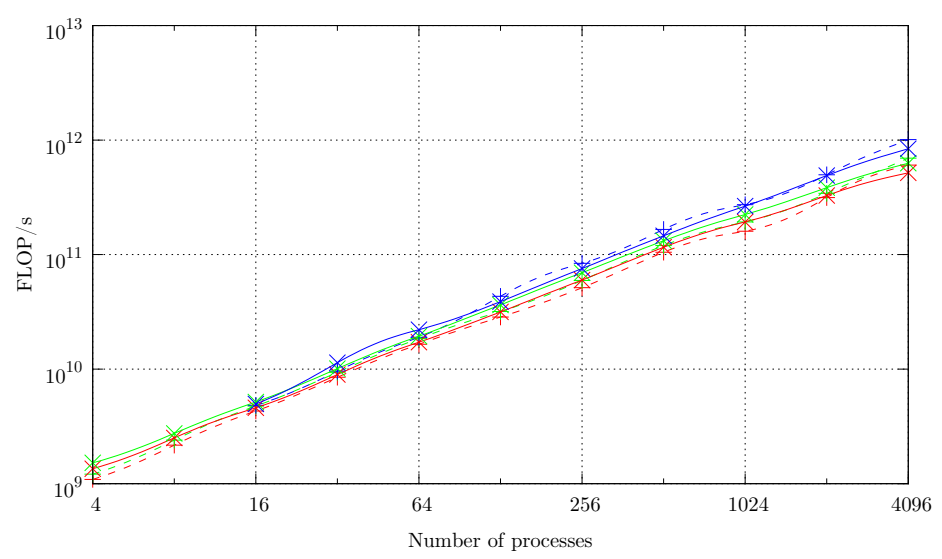

(b) Blue Gene

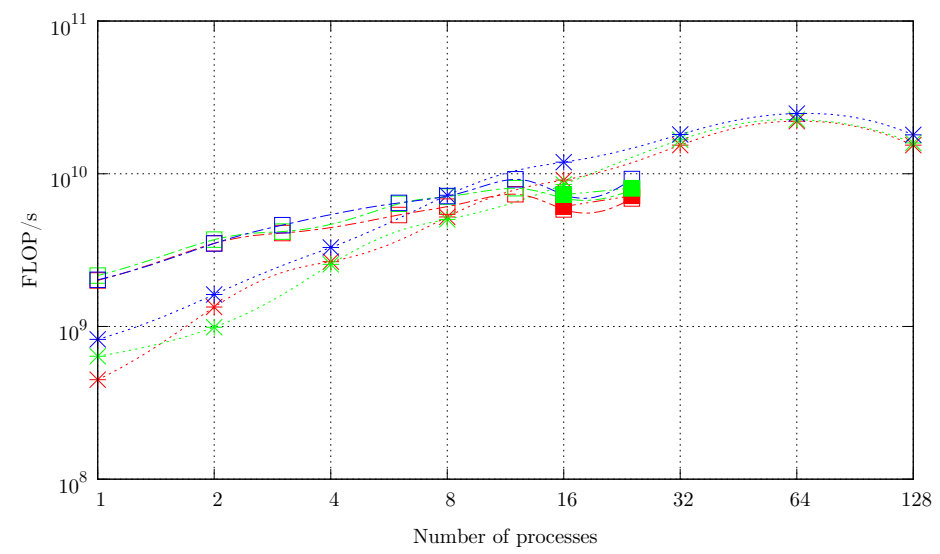

(c) Others

Figure 6.7: Total combined results of all three tests, plotted for the whole ensemble. Symbols as on page 105. 


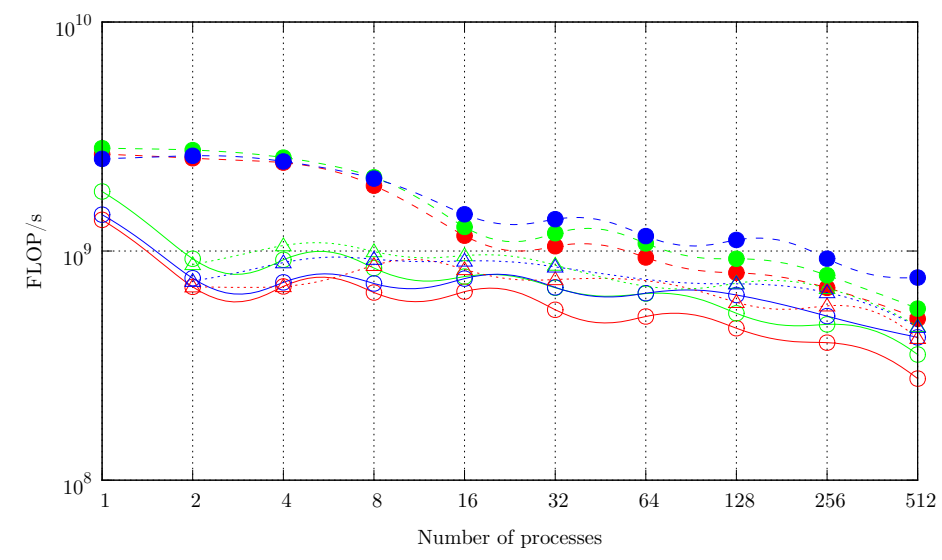

(a) HPC Wales and BlueIce2

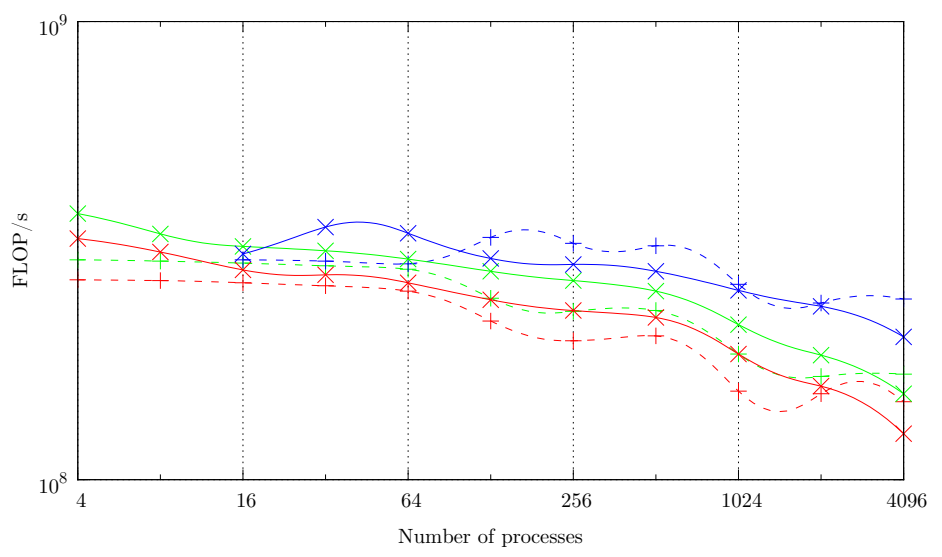

(b) Blue Gene

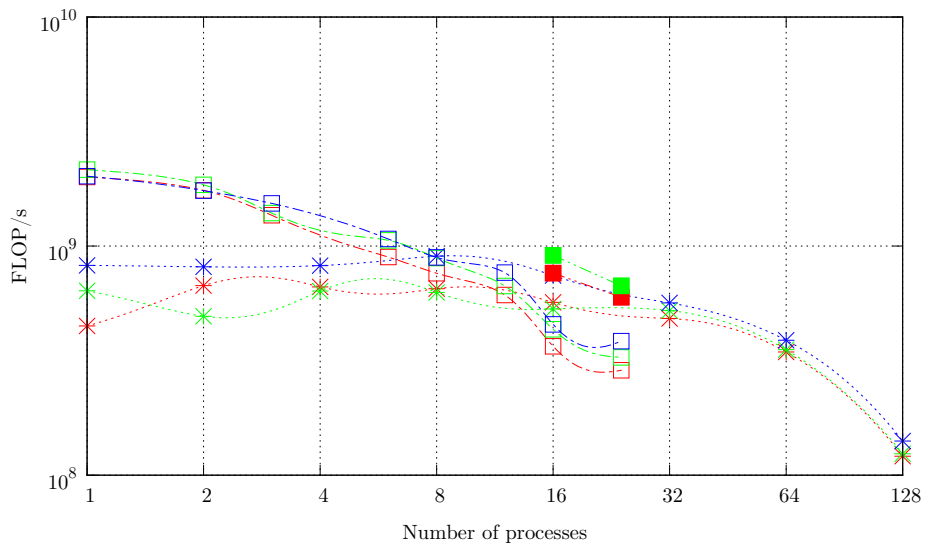

(c) Others

Figure 6.8: Total combined results of all three tests, plotting the average performance per MPI process. Symbols as on page 105. 


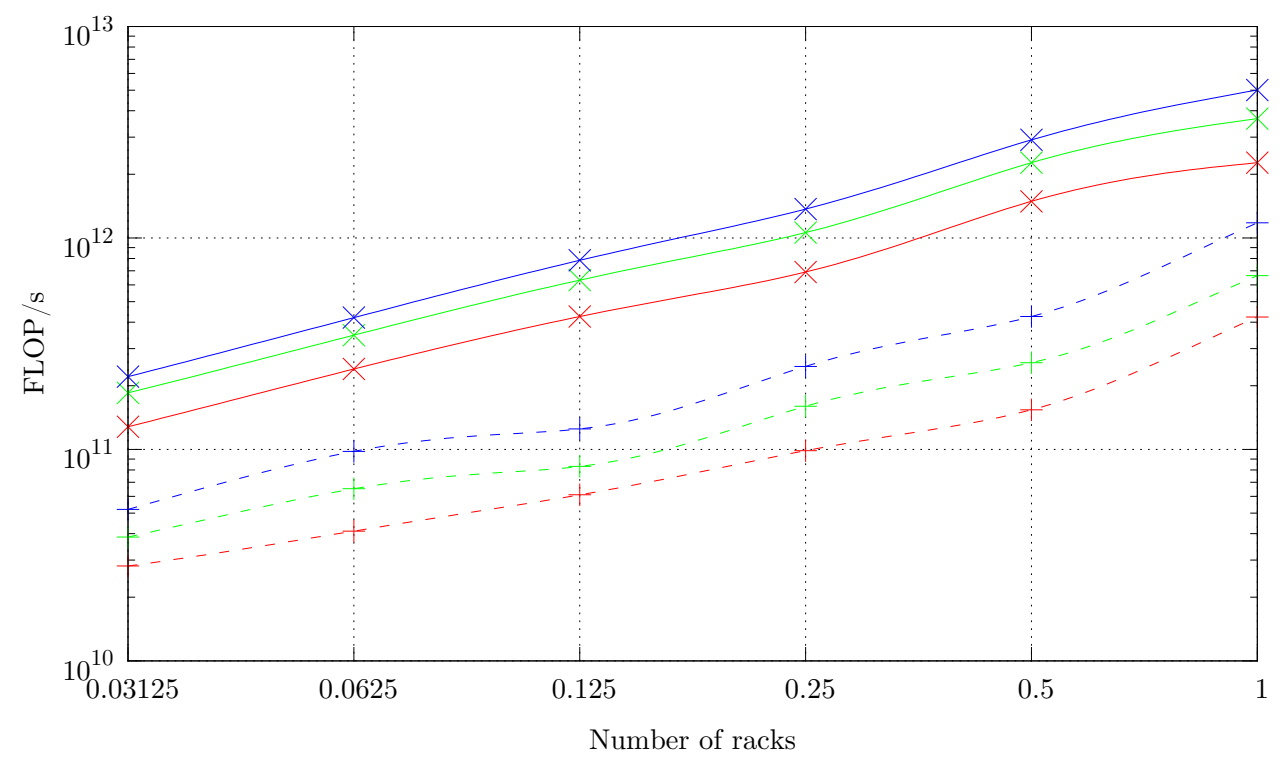

Figure 6.9: Rack-for-rack comparison between Blue Gene/P and Blue Gene/Q performance, using a larger lattice size in the case of the Blue Gene/Q to allow use of a full rack. Symbols as on page 105 .

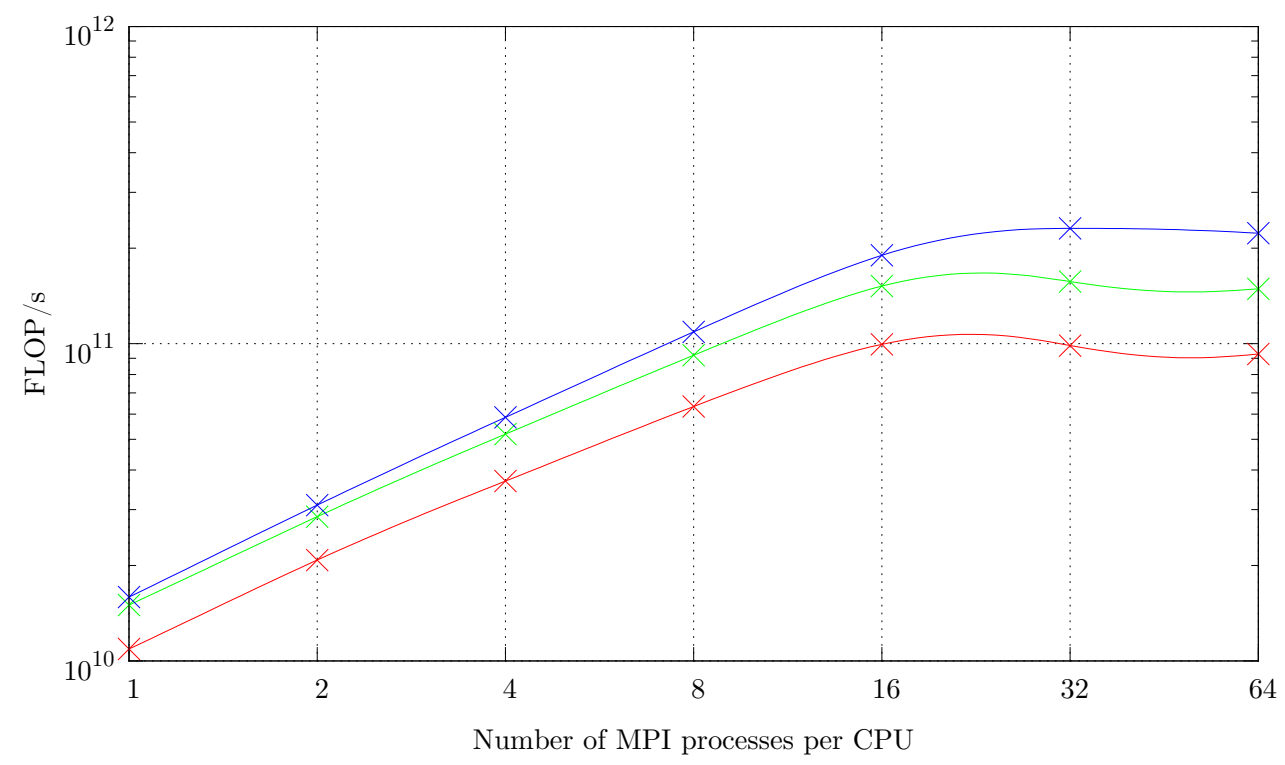

Figure 6.10: Performance comparison of a single Blue Gene/Q partition at various subdivisions; from one process per CPU through to 64 processes per CPU (four per core). Symbols as on page 105 . 


\section{HIREP CODE AND BSMBENCH}

Gene/Q. This is most likely due to the lattice geometry aligning fortuitously with the 3D torus network - it would not be seen on Blue Gene/Q due to the different network topology and core density. No tuning of the process mapping was performed for the tests; the default alignments were used. More careful choice of the alignments may be able to give a similar (or better) speedup to other partition sizes, and it is likely that a vendor looking to showcase their results with this benchmark would seek to do this.

The threading results on Blue Gene/Q are not unexpected. Since HiRep and BSMBench are single-threaded, subdividing the processors by core is equivalent to adding more cores to the problem. A significant speedup from multithreading is not expected: since multithreading only allows efficient context switching between threads rather than parallel execution (which is what multiple cores achieve), a large gain would only be possible if the code spent much of its time idle, which is not generally the case. A small gain can be found in a compute-intensive theory by having two processes rather than one per core; since this gain is effectively "free" there is no reason not to use it, unless the resulting local lattice is too small - in which case the extra overheads will outweigh the gains - or there are no more directions available to parallelise.

The Mac Pro system at 16 and 24 processes is also multithreading; notice that the performance at 16 processes drops to approximately that of 8 processes. This occurs for two reasons: firstly, the threads are localised to a single core; they cannot float between the cores and use whatever slack compute capacity there is. Secondly, the code has relatively frequent barriers to allow communication between the processes. These two factors have the effect of forcing the code to operate in lock-step on all cores, including those with only one task allocated, making the program run as slowly as if all cores had two threads running. Since the code has little idle time (even less than on the Blue Gene, since here there are no interconnect delays at all), no performance gains over 12 processes are seen at 24 processes. To test the lock-step theory, comms and balance tests $^{1}$ were performed with two 8- and 12-process runs in parallel with each other (for a total of 16 and 24 processes), and their FLOP counts added. This showed a slight enhancement over the single-run results, suggesting that the requirement to synchronise between processes does indeed reduce the potential gains from multithreading.

\footnotetext{
${ }^{1}$ The compute test required more memory than was present in the Mac Pro, so the results were not of interest.
} 


\subsection{BSMBench}

The HPC Wales machines, in particular the Sandy Bridge system, show an increasing separation between the three tests as the number of processes is increased. This nicely illustrates the assertion made earlier that the tests differ in their communications versus compute demands: the splitting begins once the processes no longer fit on the node, so must begin to use the interconnects, and increase as the interconnects are relied upon more. The slowdown is least severe for the compute, and most severe for the comms test, as we expect. This is not observed on the Blue Gene machines, whose architecture is designed from the ground up to be massively parallel, hence their advanced network topology and very high-speed interconnects. In the case of ULGQCD, the interconnects are too slow to make an informed judgement; however, the expected drop-off in performance is seen once the node size is exceeded.

The BlueIce2 system is very similar to the HPC Wales Westmere system in its makeup; their performance results are unsurprisingly also close to each other. The performance of BlueIce2 in the intermediary regime (between 16 cores where the job no longer fits on a single node, up to 256 cores) sits above the performance of the HPC Wales machine - this appears somewhat surprising, since its processors are slightly slower. In fact this enhancement is due to the job's parallelisation: on BlueIce2 it was possible to parallelise with 8 cores per node, and $N_{\text {proc }} / 8=2^{n}$ nodes, whereas allocating a given number of cores on HPC Wales generally returns the cores on the minimum number of nodes. Since the lattice is parallelised in divisions of 2, keeping the powers of 2 in the parallelisation improves the communications performance significantly; this is notable in that the comms test is the one gaining the largest performance boost on BlueIce2. Confirming this explanation is the downtick in performance (to slightly below HPC Wales) at 512 cores: since BlueIce2 only has 50 nodes, the neat power-oftwo parallelisation is no longer possible at the highest number of cores, meaning the communications speed enhancement is lost.

Potential extensions to this project would be to implement a size-independent test, where the lattice size is chosen based on the machine size. Since the total number of FLOPs is proportional to the lattice volume, a reference figure could be given for a base $4^{4}$ lattice, and multiplied up to the machine size. Results compared to the current version would be approximately comparable. It would be beneficial to find a small set of observables that would allow full characterisation of a machine's performance, to enable ranking of machines as in the TOP500 ranking (since comparison of graphs, 


\section{HIREP CODE AND BSMBENCH}

while enlightening, is time-consuming). Finding out what classes of HPC use are characterised by which test would also expand the benchmark's utility. Finally, many benchmarks now come with the ability to analytically estimate the performance on a given machine from the machine's design characteristics; such a model for BSMBench would be a powerful tool for designing new systems, in particular once other codes' performance can be modelled in terms of BSMBench's tests. 


\section{Chapter 7}

\section{Conclusions}

In the work presented in this thesis, we have explored a number of aspects of strong dynamics beyond the Standard Model. Chapter 4 presented the first lattice study of the $\mathrm{SU}(2)$ Adj Nf1 theory. After a description of how the theory can be viewed as a theory of two Majorana fermions, we observed the spectroscopy of the theory (looking at the pseudoscalar, scalar, vector, and axial vector mesons, the spin- $\frac{1}{2}$ state, and the $0^{++}$ glueball), its string tension from both Wilson and Polyakov loops, and the anomalous dimension of the fermionic condensate from the Dirac mode number.

The spectroscopy had interesting behaviour: when normalised by the square root of the string tension, all spectral observables studied (including the pseudoscalar, scalar, vector, and axial vector mesons, the spin- $\frac{1}{2}$ state, and the $0^{++}$glueball) were approximately constant in their masses throughout the observed regions, although the nonmesonic states showed slight inconsistency with the constant value at higher fermion mass. These results are inconsistent with traditional QCD-like confining behaviour, indicating a theory within or close to the conformal window; a desirable property for a candidate Walking Technicolor model. The anomalous dimension was found to be large, $0.9 \lesssim \gamma_{*} \lesssim 0.95$, another important feature of a Walking Technicolor candidate.

In chapter 5 we gave a look to the topology of two theories, SU(2)Adj Nf1 and Minimal Walking Technicolor, both $\mathrm{SU}(2)$ theories with one and two adjoint Dirac flavours respectively. The topological susceptibility and instanton size distribution were calculated, and compared to those of pure gauge $\mathrm{SU}(2)$; the results were found to be consistent between the three cases. This again is indicative of the fermion degrees of freedom becoming dynamically quenched, reaffirming the conclusion of chapter 4 


\section{CONCLUSIONS}

(and in the case of Minimal Walking Technicolor, earlier studies) that the theory is (near-)conformal.

In chapter 6 I presented the BSMBench benchmarking tool, developed from the HiRep code (which was used for all the simulations described in the preceding chapters). This tool may be used to influence machine procurement decisions for BSM physics, and also has applications outside physics, since its unique ability to adjust the ratio of communications to compute demands allows it flexibility to emulate the demand ratios of other applications - something which is not possible in most benchmarks. A number of machines' performance were compared.

There are many exciting avenues for future work developing from this project. For the SU(2)Adj Nf1 theory, it would be beneficial to extend the study to lower fermion mass (through larger lattices), and also to higher $\beta$ (to investigate both the continuum and chiral limits). It would also be interesting to extract the states that were ignored in this study, and to look at the running of the coupling. At larger lattice size it may also be possible to extract the anomalous dimension from topological observables, which would give an independent estimate to compare with that found from the Dirac mode number. Exploring other theories with these methods would also be interesting. For example, since SU(2)Adj Nf1 has too few Goldstone states to be a viable Technicolor candidate, but has a high anomalous dimension and is in or near the conformal window, and Minimal Walking Technicolor has more Goldstone states than is necessary and a lower anomalous dimension than would be desirable for a Technicolor candidate theory, it would be exciting to look at the theory with three Majorana flavours (lying directly between the two Majorana flavours of SU(2)AdjNf1 and the four of Minimal Walking Technicolor).

The benchmark is also ripe for improvement. It is currently limited in the maximum machine size it can test, since it uses a single lattice size. This restriction could be lifted by instead choosing a single local lattice size - multiplying by the number of processes to obtain the total lattice size. Developing an analytical model of the benchmark's performance would allow it to have influence into machine design decisions rather than just purchasing ones. If it were possible to distill a single number for a machine's performance in a given test rather than one per partition size, comparison between machines would be easier and ranking (as is done in the TOP500 and Graph500 benchmarks) would be possible. Finally, studying which other numerical 
problems have similar demands to the tests available in the benchmark would allow easy application of the benchmark to other fields beyond lattice physics. 
7. CONCLUSIONS 


\section{Appendix A}

\section{Visualisations of Topological Charge Distributions}

A number of visualisations were carried out on the topological charge distribution of a single $64 \times 32^{3}$ lattice configuration of SU(2)Adj Nf1 at $\beta=2.05, m=-1.51$, using the VisIt visualisation tool $[174,175]$. Positive and negative topological charge are shown in orange and blue respectively.

In figure A.1 a single timeslice is shown both before cooling and after 20 cools. Both cases are normalised by the maxima in the distribution; in spite of this, the gauge noise is clearly dominating in the uncooled case. In the cooled case, the individual instantons can be seen easily. Figure A.2 shows another timeslice, this time showing each cooling step in addition to the uncooled case. Figure A.3 adds in the time dimension: each cube is still a 3-dimensional time slice, and the time dimension runs around the ring. Moving down the columns gives the successively cooled versions of the same time slice, up to 20 cools. The removal of the noise is still clearly visible, and the plateau mentioned in chapter 5 between 10 and 20 cools that allows relative flexibility in the choice of cooling can be observed in the lower half of the image. This image was submitted to Swansea University's Research as Art and the Royal Society's Picturing Science events, and was selected as the winner of the Postgraduate Award at the former. 


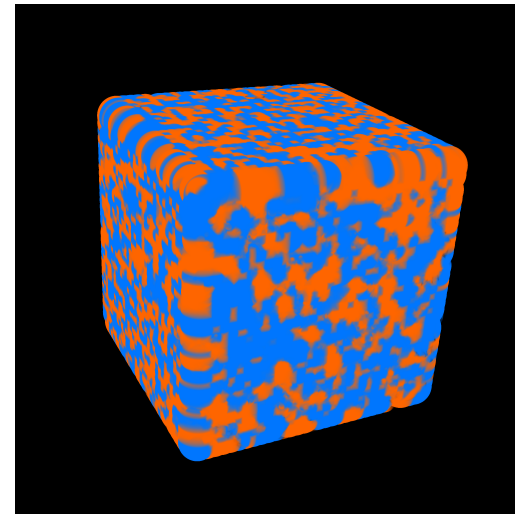

(a) Before cooling.

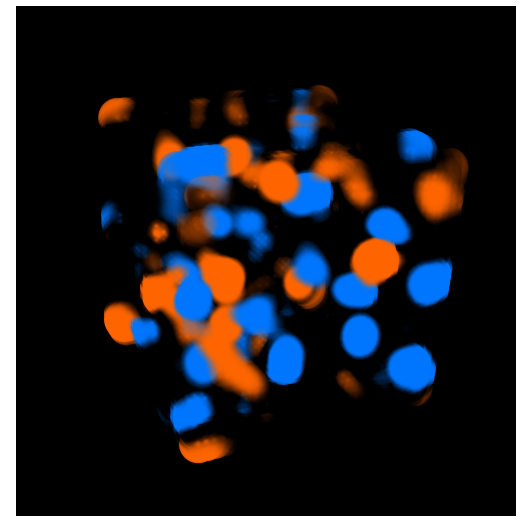

(b) After 20 cools.

Figure A.1: The topological charge distribution of a single timeslice, before and after cooling. Rendering was performed using a Mac Pro machine in Swansea University as the frontend, and the BlueIce2 cluster in Swansea University as the backend, using 480 compute cores across 40 nodes.

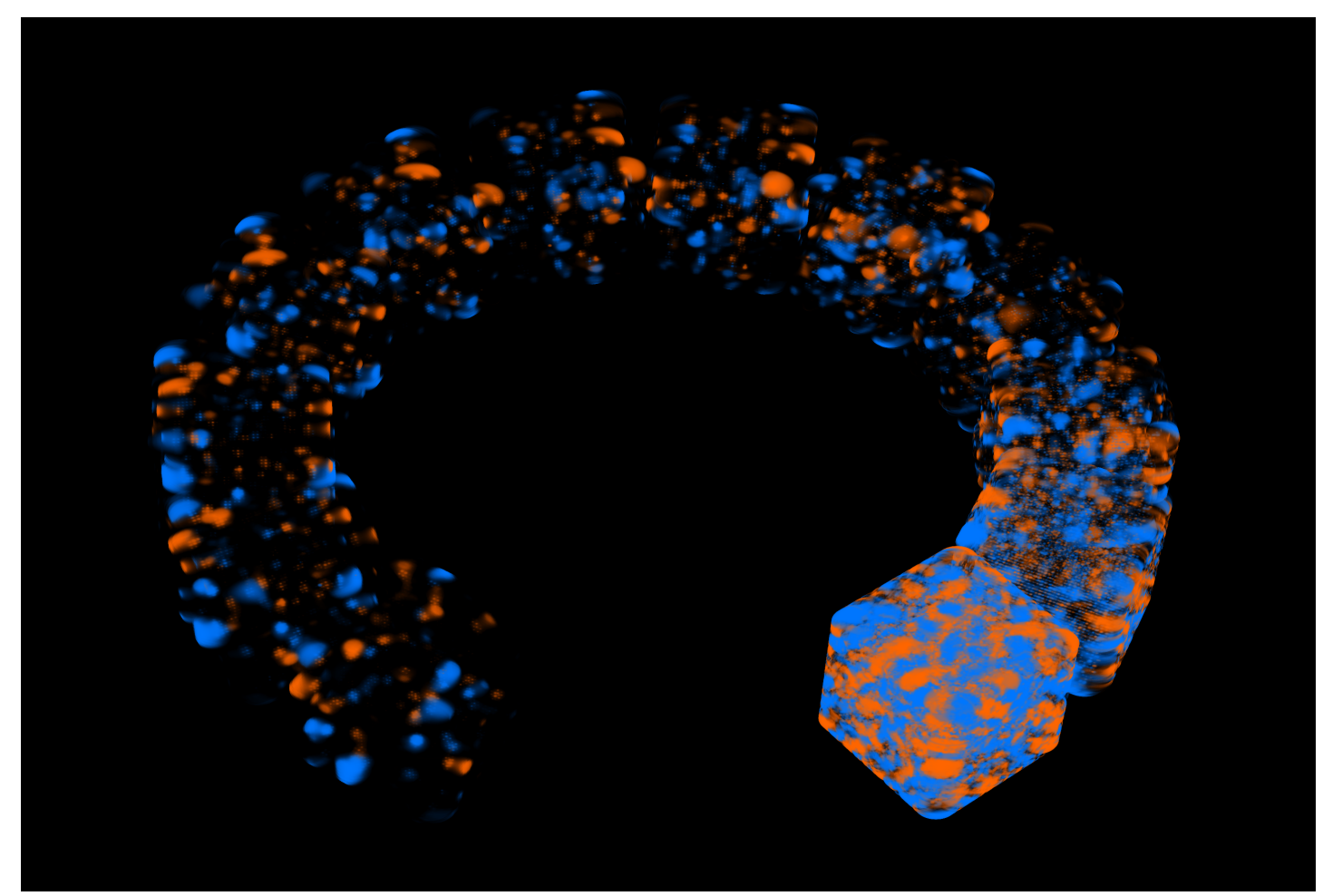

Figure A.2: The topological charge distribution of a single timeslice. Moving anticlockwise around the ring takes us from the uncooled case to 10 cools. Rendering was performed using the Mac Pro used in figure A.1 as both front and backend. 


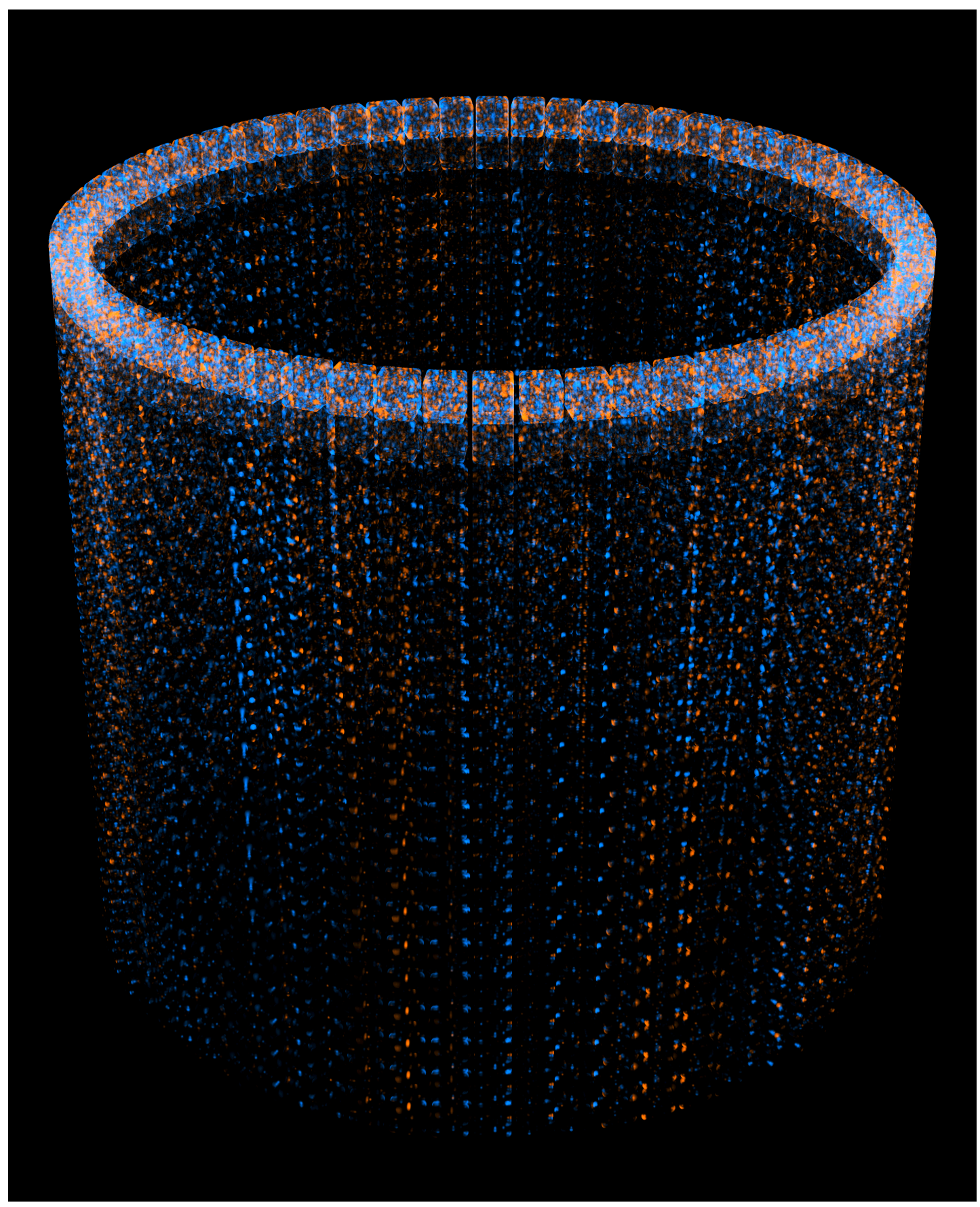

Figure A.3: The topological charge distribution of all 64 timeslices of the configuration. Moving around the ring takes us around the time dimension, while moving down the columns takes us from the uncooled state to 20 cools. This gives a total of 1344 cubes. Rendering was performed using the machine combination used for figure A.1. 
A. VISUALISATIONS OF TOPOLOGICAL CHARGE DISTRIBUTIONS 


\section{Appendix B}

\section{README and LICENSE files for BSMBench}

\section{B.1 README}

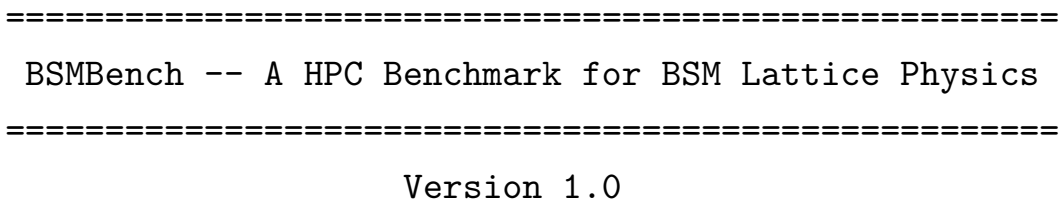

\section{Contents}

$========$

1. Compiling BSMBench

1.1 Machine configuration files

1.2 Compilation script

2. Running BSMBench

3. Interpreting output

4. Version history

1. Compiling BSMBench 


\section{B. README AND LICENSE FILES FOR BSMBENCH}

1.1 Machine configuration file

This file specifies your $C$ compiler and its flags. The syntax is

$$
\begin{aligned}
& \mathrm{CC}=[\mathrm{C} \text { compiler }] \\
& \text { CFLAGS }=[\text { flags }]
\end{aligned}
$$

Some examples are given in the machine-config directory. The files BGP.cfg and BGP-1.cfg contain the parameters used for the Blue Gene/P performance comparison data, which have been obtained running the code with the environment variable DCMF_INTERRUPT set to 1.

\subsection{Compilation script}

By default, the compilation script compiles only MPI versions of the benchmark. In this case it takes a single parameter: the machine configuration file. For example

./make.sh machine-config/generic.cfg

will compile a version suitable for a generic cluster.

If a second parameter is included, it is used as the machine configuration file for a non-MPI version. In this case, two sets of executables are generated, one ending in _1cpu. For example

./make.sh machine-config/BGP.cfg machine-config/BGP-1.cfg

will generate MPI and non-MPI files for a Blue Gene/P machine.

To avoid generating the MPI versions, set the NO_MPI environmental variable and pass a dummy first parameter. For example

export NO_MPI=YES 
./make.sh xxxx machine-config/OSX-1.cfg

will generate non-MPI executables only for a machine running Mac OS X.

Three files are generated in each set:

- bsmbench_comms[_1cpu]: Runs the communications test

- bsmbench_balance[_1cpu]: Runs the balanced test

- bsmbench_compute[_1cpu]: Runs the compute test

\section{Running BSMBench}

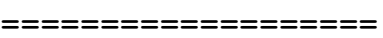

Before running a test with BSMBench, you must know:

- which test you want to run (comms, balance, or compute)

- how many processes you want to use

BSMBench is single-threaded, so ideally the number of processes should be equal to the number of cores (for example, Virtual Node mode on Blue Gene/P), or potentially more if the machine has multithreading cores.

The general syntax for running BSMBench is then:

./bsmbench_[test]\{_1cpu\}-i [input_file] -o [output file]

Input files are located in the sets directory. They are named systematically as [test]-[number-of-processes].bsmbench. The output directory is provided for convenience as a location to put output files.

For example, to run the balance test on a single core, the syntax could be ./bsmbench_balance_1cpu -i sets/balance-1.bsmbench -o output/bal-1.out

To run the tests in parallel, mpirun must be used. For example, to run the compute test on 64 cores, the syntax could be 


\section{B. README AND LICENSE FILES FOR BSMBENCH}

mpirun -np $64 . /$ bsmbench_compute -i sets/compute-64.bsmbench

-o output/compute-64.out

mpirun can then be called by your queueing system of choice.

On Blue Gene machines please export the environmental variable:

DCMF_INTERRUPT $=1$

3. Interpreting output

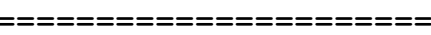

Output files start with some general remarks about the parameters used, marked with [MAIN], then move on to the benchmark results, marked with [BENCH]. To pull out the relevant results, grep can be used:

grep BENCH [output-file]

First, an indication is given of whether the precision test was successful.

Then, for each performance test, the output consists of:

- the time taken

- the average FLOP/s rating for the test

- the average FLOP/s per process for the test

- (Dphi only) a relative comparison to the speed of a Blue Gene/P machine performing the same test

Following this, an indication of success is given, followed by the average FLOP/s and FLOP/s per process across all tests, and a relative comparison to the speed of a Blue Gene/P machine performing the same test.

Of the tests, the Dphi is the most relevant for performing lattice calculations, and for testing communications bandwidth, so results from this test are compared with reference machines separately from the total aggregate figure. It is recommended that this figure be weighted most 
strongly in any comparison.

4. Version History

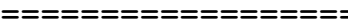

Version 1.0 (2012-04-19)

- Initial release

\section{B.2 LICENSE}

Copyright (c) 2012, Claudio Pica, Agostino Patella, Antonio Rago, Luigi Del Debbio, Biagio Lucini, Edward Bennett.

All rights reserved.

Redistribution and use in source and binary forms, with or without modification, are permitted provided that the following conditions are met:

1) Redistributions of source code must retain the above copyright notice, this list of conditions and the following disclaimer.

2) Redistributions in binary form must reproduce the above copyright notice,

this list of conditions and the following disclaimer in the documentation and/or other materials provided with the distribution.

3) Any publication in any form derived from the use of this software or any modification of it must refer explicitly to the original BSMBench package (including the official URL http://github.com/blucini/BSMBench) and cite the following two publications:

[1] L. Del Debbio, A. Patella, C. Pica, "Higher representations on the lattice: Numerical simulations. SU(2) with adjoint fermions", Phys. Rev. D81 (2010) 094503, DOI 10.1103/PhysRevD.81.094503

[2] L. Del Debbio, B. Lucini, A. Patella, C. Pica, A. Rago, "Conformal versus confining scenario in SU(2) with adjoint fermions", Phys.Rev. D80 (2009) 074507, DOI 10.1103/PhysRevD.80.074507

4) The names of the contributors may be used to endorse or promote 


\section{B. README AND LICENSE FILES FOR BSMBENCH}

products derived from this software without specific prior written permission.

THIS SOFTWARE IS PROVIDED BY THE COPYRIGHT HOLDERS AND CONTRIBUTORS "AS IS" AND ANY EXPRESS OR IMPLIED WARRANTIES, INCLUDING, BUT NOT LIMITED TO, THE IMPLIED WARRANTIES OF MERCHANTABILITY AND FITNESS FOR A PARTICULAR PURPOSE ARE DISCLAIMED. IN NO EVENT SHALL THE COPYRIGHT HOLDER OR CONTRIBUTORS BE LIABLE FOR ANY DIRECT, INDIRECT, INCIDENTAL, SPECIAL, EXEMPLARY, OR CONSEQUENTIAL DAMAGES (INCLUDING, BUT NOT LIMITED TO, PROCUREMENT OF SUBSTITUTE GOODS OR SERVICES; LOSS OF USE, DATA, OR PROFITS; OR BUSINESS INTERRUPTION) HOWEVER CAUSED AND ON ANY THEORY OF LIABILITY, WHETHER IN CONTRACT, STRICT LIABILITY, OR TORT (INCLUDING NEGLIGENCE OR OTHERWISE) ARISING IN ANY WAY OUT OF THE USE OF THIS SOFTWARE, EVEN IF ADVISED OF THE POSSIBILITY OF SUCH DAMAGE. 


\section{References}

[1] M. Piai, "Lectures on walking technicolor, holography and gauge/gravity dualities," Adv. High Energy Phys. 2010 (2010) 464302, arXiv:1004.0176 [hep-ph]. iv, 8, 17

[2] T. Pickup, Investigating the Conformal Window of $\mathrm{SU}(N)$ Gauge Theories. $\mathrm{PhD}$ thesis, University of Oxford, 2011. iv

[3] PACS-CS Collaboration Collaboration, S. Aoki et al., " $2+1$ Flavor Lattice QCD toward the Physical Point," Phys.Rev. D79 (2009) 034503, arXiv:0807.1661 [hep-lat]. vi, 42

[4] R. Dowdall, C. Davies, T. Hammant, and R. Horgan, "Precise heavy-light meson masses and hyperfine splittings from lattice QCD including charm quarks in the sea," Phys. Rev. D86 (2012) 094510, arXiv:1207.5149 [hep-lat]. vi, 42, 43

[5] ETM Collaboration Collaboration, C. Alexandrou et al., "Low-lying baryon spectrum with two dynamical twisted mass fermions," Phys.Rev. D80 (2009) 114503, arXiv:0910.2419 [hep-lat]. vi, 42, 43

[6] S. Bethke, "World Summary of $\alpha_{s}$ (2012)," arXiv:1210.0325 [hep-ex]. vi, 42, 44

[7] E. T. Neil, "Exploring Models for New Physics on the Lattice," PoS LATTICE2011 (2011) 009, arXiv:1205.4706 [hep-lat]. vi, 44, 45

[8] B. Lucini and M. Teper, "SU(N) gauge theories in four-dimensions: Exploring the approach to $N=\infty$," JHEP 0106 (2001) 050, arXiv: hep-lat/0103027 [hep-lat]. vii, 81,82 


\section{REFERENCES}

[9] T. Schäfer and E. V. Shuryak, "Instantons in QCD," Rev. Mod. Phys. 70 (Apr, 1998) 323-425, arXiv:hep-ph/9610451. vii, 75, 81

[10] L. Del Debbio, B. Lucini, A. Patella, C. Pica, and A. Rago, "Infrared dynamics of Minimal Walking Technicolor," Phys. Rev. D82 (Jul, 2010) 014510, arXiv: 1004.3206. ix, 82, 85, 86, 96

[11] P. W. Higgs, "Broken symmetries, massless particles and gauge fields," Phys. Lett. 12 (1964) 132-133. 1, 5

[12] P. W. Higgs, "Broken Symmetries and the Masses of Gauge Bosons," Phys. Rev. Lett. 13 (1964) 508-509. 1, 5

[13] E. Bennett, B. Lucini, and A. Patella, "SU(2) with one Dirac flavour in the adjoint representation," in Proceedings of Strong and Electroweak Matter (SEWM) 2012. 2012.

http://pyweb.swan.ac.uk/sewm/sewmweb/posters/bennett.pdf. 2

[14] E. Bennett and B. Lucini, "Topology of Minimal Walking Technicolor," Eur. Phys. J. C73 no. 5, (2013) 1-7, arXiv:1209.5579.

http://dx.doi.org/10.1140/epjc/s10052-013-2426-6. 2, 96

[15] E. Bennett, B. Lucini, K. Jordan, A. Patella, C. Pica, and A. Rago, "BSMBench: A HPC benchmark for beyond the standard model lattice physics," in Proceedings of the International Supercomputing Conference 2012. 2012. http://pyweb.swan.ac.uk/ pyeb/superposter.pdf. 2, 97

[16] S. Laporta and E. Remiddi, "The Analytical value of the electron $(g-2)$ at order $\alpha^{3}$ in QED," Phys. Lett. B379 (1996) 283-291, arXiv:hep-ph/9602417 [hep-ph]. 3

[17] T. Aoyama, M. Hayakawa, T. Kinoshita, and M. Nio, "Tenth-Order QED Contribution to the Electron g-2 and an Improved Value of the Fine Structure Constant," Phys. Rev. Lett. 109 (2012) 111807, arXiv:1205.5368 [hep-ph]. 3

[18] Particle Data Group Collaboration, J. Beringer et al., "Review of particle physics," Phys. Rev. D86 (Jul, 2012) 010001. 3, 5, 14 
[19] ATLAS Collaboration, The ATLAS Collaboration, "Observation of a new particle in the search for the Standard Model Higgs boson with the ATLAS detector at the LHC," Phys. Lett. B716 no. 1, (2012) 1-29, arXiv:1207.7214. 3

[20] CMS Collaboration, The CMS Collaboration, "Observation of a new boson at a mass of $125 \mathrm{GeV}$ with the CMS experiment at the LHC," Phys. Lett. B716 no. 1, (2012) $30-61$, arXiv:1207.7235. 3

[21] S. Weinberg, "A Model of Leptons," Phys. Rev. Lett. 19 (1967) 1264-1266. 5

[22] A. Salam, "Weak and Electromagnetic Interactions," Conf. Proc. $\mathbf{6 6 8 0 5 1 9}$ (1968) 367-377. 5

[23] S. Glashow, "Partial Symmetries of Weak Interactions," Nucl. Phys. B22 (1961) 579-588. 5

[24] F. Englert and R. Brout, "Broken Symmetry and the Mass of Gauge Vector Mesons," Phys. Rev. Lett. 13 (1964) 321-323. 5

[25] G. Guralnik, C. Hagen, and T. Kibble, "Global Conservation Laws and Massless Particles," Phys. Rev. Lett. 13 (1964) 585-587. 5

[26] J. Goldstone, "Field Theories with Superconductor Solutions," Nuovo Cim. 19 (1961) 154-164. 5

[27] M. E. Peskin and D. V. Schroeder, "An Introduction to quantum field theory,". 5

[28] S. Weinberg, "Implications of Dynamical Symmetry Breaking," Phys. Rev. D13 (1976) 974-996. 7

[29] S. Weinberg, "Implications of Dynamical Symmetry Breaking: An Addendum," Phys. Rev. D19 (1979) 1277-1280. 7

[30] L. Susskind, "Dynamics of Spontaneous Symmetry Breaking in the Weinberg-Salam Theory," Phys. Rev. D20 (1979) 2619-2625. 7

[31] E. Farhi and L. Susskind, "Technicolor," Phys. Rept. 74 (1981) 277. 7 


\section{REFERENCES}

[32] J. Bell and R. Jackiw, "A PCAC puzzle: pi0 $\rightarrow$ gamma gamma in the sigma model," Nuovo Cim. A60 (1969) 47-61. 8

[33] S. L. Adler, "Axial vector vertex in spinor electrodynamics," Phys. Rev. D177 (1969) 2426-2438. 8

[34] M. Kaku, Quantum field theory: A Modern introduction. Oxford University Press, 1993. 8

[35] T. Appelquist and G.-H. Wu, "The Electroweak chiral Lagrangian and new precision measurements," Phys. Rev. D48 (1993) 3235-3241, arXiv:hep-ph/9304240 [hep-ph]. 8

[36] T. Appelquist and G.-H. Wu, "The Electroweak chiral Lagrangian and CP violating effects in technicolor theories," Phys. Rev. D51 (1995) 240-250, arXiv:hep-ph/9406416 [hep-ph]. 8

[37] D. D. Dietrich and F. Sannino, "Conformal window of $\mathrm{SU}(N)$ gauge theories with fermions in higher dimensional representations," Phys. Rev. D75 (2007) 085018, arXiv:hep-ph/0611341 [hep-ph]. 9, 17, 42, 46, 47

[38] W. E. Caswell, "Asymptotic Behavior of non-Abelian Gauge Theories to Two Loop Order," Phys. Rev. Lett. 33 (1974) 244. 9, 10

[39] D. Jones, "The Two Loop beta Function for a $G(1) \times G(2)$ Gauge Theory," Phys. Rev. D25 (1982) 581. 9

[40] T. Banks and A. Zaks, "On the Phase Structure of Vector-Like Gauge Theories with Massless Fermions," Nucl. Phys. B196 (1982) 189. 10

[41] T. Appelquist, K. D. Lane, and U. Mahanta, "On the ladder approximation for spontaneous chiral symmetry breaking," Phys. Rev. Lett. 61 (1988) 1553. 10

[42] A. G. Cohen and H. Georgi, "Walking beyond the rainbow," Nucl. Phys. B314 (1989) 7. 10, 11, 12

[43] R. Fukuda and T. Kugo, "Schwinger-Dyson Equation for Massless Vector Theory and Absence of Fermion Pole," Nucl. Phys. B117 (1976) 250. 12 
[44] S. Dimopoulos and L. Susskind, "Mass Without Scalars," Nucl. Phys. B155 (1979) 237-252. 13

[45] E. Eichten and K. D. Lane, "Dynamical Breaking of Weak Interaction Symmetries," Phys. Lett. B90 (1980) 125-130. 13, 14

[46] S. Raby, S. Dimopoulos, and L. Susskind, "Tumbling Gauge Theories," Nucl.Phys. B169 (1980) 373. 14

[47] S. Chadha and M. E. Peskin, "Implications of chiral dynamics in theories of technicolor. 2. The mass of the $P^{+}, "$ Nucl. Phys. B187 (1981) 541. 14

[48] J. Sanz-Cillero, "Pion and kaon decay constants: Lattice versus resonance chiral theory," Phys. Rev. D70 (2004) 094033, arXiv:hep-ph/0408080 [hep-ph]. 14

[49] R. S. Chivukula and E. H. Simmons, "Condensate Enhancement and D-Meson Mixing in Technicolor Theories," Phys. Rev. D82 (2010) 033014, arXiv:1005.5727 [hep-lat]. 14

[50] B. Holdom, "Techniodor," Phys. Lett. B150 (1985) 301. 15

[51] K. Yamawaki, M. Bando, and K.-i. Matumoto, "Scale Invariant Technicolor Model and a Technidilaton," Phys. Rev. Lett. 56 (1986) 1335.

[52] T. W. Appelquist, D. Karabali, and L. Wijewardhana, "Chiral Hierarchies and the Flavor Changing Neutral Current Problem in Technicolor," Phys. Rev. Lett. 57 (1986) 957. 15

[53] D. Elander and M. Piai, "The decay constant of the holographic techni-dilaton and the $125 \mathrm{GeV}$ boson," arXiv:1208.0546 [hep-ph]. 17, 18

[54] N. Evans and K. Tuomine, "Holographic Modelling of a Light Techni-Dilaton," arXiv: 1302.4553 [hep-ph]. 17

[55] S. Matsuzaki and K. Yamawaki, "Holographic techni-dilaton at $125 \mathrm{GeV}$," Phys. Rev. D86 (2012) 115004, arXiv:1209.2017 [hep-ph]. 17, 18

[56] CMS Collaboration, S. Chatrchyan et al., "Observation of a new boson with mass near $125 \mathrm{GeV}$ in pp collisions at $\sqrt{s}=7$ and $8 \mathrm{TeV}$," arXiv: 1303.4571 [hep-ex]. 17 


\section{REFERENCES}

[57] R. S. Chivukula, "Lectures on technicolor and compositeness," arXiv:hep-ph/0011264 [hep-ph]. 17

[58] K. Lane, "Two lectures on technicolor," arXiv:hep-ph/0202255 [hep-ph].

[59] C. T. Hill and E. H. Simmons, "Strong dynamics and electroweak symmetry breaking," Phys. Rept. 381 (2003) 235-402, arXiv:hep-ph/0203079 [hep-ph].

[60] A. Martin, "Technicolor Signals at the LHC," arXiv:0812.1841 [hep-ph].

[61] F. Sannino, "Conformal Dynamics for TeV Physics and Cosmology," Acta Phys. Polon. B40 (2009) 3533-3743, arXiv:0911.0931 [hep-ph]. 17

[62] N. Seiberg, "Electric - magnetic duality in supersymmetric non-Abelian gauge theories," Nucl. Phys. B435 (1995) 129-146, arXiv:hep-th/9411149 [hep-th]. 18

[63] C. D. Carone, J. Erlich, and J. A. Tan, "Holographic Bosonic Technicolor," Phys. Rev. D75 (2007) 075005, arXiv:hep-ph/0612242 [hep-ph]. 18

[64] D. Elander and M. Piai, "Light scalars from a compact fifth dimension," JHEP 1101 (2011) 026, arXiv:1010.1964 [hep-th].

[65] M. Fabbrichesi, M. Piai, and L. Vecchi, "Dynamical electro-weak symmetry breaking from deformed AdS: Vector mesons and effective couplings," Phys. Rev. D78 (2008) 045009, arXiv:0804.0124 [hep-ph].

[66] M. Goykhman and A. Parnachev, "S-parameter, Technimesons, and Phase Transitions in Holographic Tachyon DBI Models," Phys. Rev. D87 (2013) 026007, arXiv:1211.0482 [hep-th].

[67] K. Haba, S. Matsuzaki, and K. Yamawaki, "S Parameter in the Holographic Walking/Conformal Technicolor," Prog. Theor. Phys. 120 (2008) 691-721, arXiv:0804.3668 [hep-ph].

[68] J. Hirn and V. Sanz, "A Negative S parameter from holographic technicolor," Phys. Rev. Lett. 97 (2006) 121803, arXiv:hep-ph/0606086 [hep-ph]. 
[69] J. Hirn and V. Sanz, "The Fifth dimension as an analogue computer for strong interactions at the LHC," JHEP 0703 (2007) 100, arXiv: hep-ph/0612239 [hep-ph].

[70] J. Hirn, A. Martin, and V. Sanz, "Describing viable technicolor scenarios," Phys. Rev. D78 (2008) 075026, arXiv:0807.2465 [hep-ph].

[71] D. K. Hong and H.-U. Yee, "Holographic estimate of oblique corrections for technicolor," Phys. Rev. D74 (2006) 015011, arXiv:hep-ph/0602177 [hep-ph].

[72] D. Kutasov, J. Lin, and A. Parnachev, "Holographic Walking from Tachyon DBI," Nucl. Phys. B863 (2012) 361-397, arXiv:1201.4123 [hep-th].

[73] D. Levkov, V. Rubakov, S. Troitsky, and Y. Zenkevich, "Constraining Holographic Technicolor," Phys. Lett. B716 (2012) 350-355, arXiv:1201.6368 [hep-ph].

[74] M. Round, "Holographic Renormalisation and the Electroweak Precision Parameters," Phys. Rev. D82 (2010) 053002, arXiv:1003.2933 [hep-ph]. 18

[75] L. Anguelova, "Electroweak Symmetry Breaking from Gauge/Gravity Duality," Nucl. Phys. B843 (2011) 429-454, arXiv:1006.3570 [hep-th]. 18

[76] L. Anguelova, P. Suranyi, and L. Wijewardhana, "Holographic Walking Technicolor from D-branes," Nucl. Phys. B852 (2011) 39-60, arXiv:1105.4185 [hep-th].

[77] L. Anguelova, P. Suranyi, and L. R. Wijewardhana, "Scalar Mesons in Holographic Walking Technicolor," Nucl. Phys. B862 (2012) 671-690, arXiv:1203.1968 [hep-th].

[78] C. D. Carone, J. Erlich, and M. Sher, "Holographic Electroweak Symmetry Breaking from D-branes," Phys. Rev. D76 (2007) 015015, arXiv:0704.3084 [hep-th].

[79] T. Clark, S. Love, and T. ter Veldhuis, "Holographic Walking Technicolor and Stability of Techni-Branes," arXiv:1208.0817 [hep-th]. 


\section{REFERENCES}

[80] T. Hirayama and K. Yoshioka, "Holographic Construction of Technicolor Theory," JHEP 0710 (2007) 002, arXiv:0705.3533 [hep-ph].

[81] O. Mintakevich and J. Sonnenschein, "Holographic technicolor models and their S-parameter," JHEP 0907 (2009) 032, arXiv:0905.3284 [hep-th]. 18

[82] L. Anguelova, P. Suranyi, and L. R. Wijewardhana, "Glueball Spectrum in a Gauge Theory with Two Dynamical Scales," arXiv:1212.1176 [hep-th]. 18

[83] D. Elander, C. Nunez, and M. Piai, "A Light scalar from walking solutions in gauge-string duality," Phys. Lett. B686 (2010) 64-67, arXiv:0908.2808 [hep-th].

[84] D. Elander, J. Gaillard, C. Nunez, and M. Piai, "Towards multi-scale dynamics on the baryonic branch of Klebanov-Strassler," JHEP 1107 (2011) 056, arXiv:1104.3963 [hep-th].

[85] C. Nunez, I. Papadimitriou, and M. Piai, "Walking Dynamics from String Duals," Int. J. Mod. Phys. A25 (2010) 2837-2865, arXiv:0812.3655 [hep-th].

[86] C. Nunez, M. Piai, and A. Rago, "Wilson Loops in string duals of Walking and Flavored Systems," Phys. Rev. D81 (2010) 086001, arXiv:0909.0748 [hep-th].

[87] S. P. Kumar, D. Mateos, A. Paredes, and M. Piai, "Towards holographic walking from $\mathscr{N}=4$ super Yang-Mills," JHEP 1105 (2011) 008, arXiv: 1012.4678 [hep-th]. 18

[88] K. G. Wilson, "Confinement of Quarks," Phys. Rev. D10 (1974) 2445-2459. 24

[89] H. B. Nielsen and M. Ninomiya, "No Go Theorem for Regularizing Chiral Fermions," Phys. Lett. B105 (1981) 219. 24

[90] K. G. Wilson, "Quarks and Strings on a Lattice,". 24

[91] J. B. Kogut and L. Susskind, "Hamiltonian Formulation of Wilson's Lattice Gauge Theories," Phys. Rev. D11 (1975) 395. 25 
[92] L. Susskind, "Lattice Fermions," Phys. Rev. D16 (1977) 3031-3039. 25

[93] Cornell-Oxford-Tel Aviv-Yeshiva Collaboration, T. Banks et al., "Strong Coupling Calculations of the Hadron Spectrum of Quantum Chromodynamics," Phys. Rev. D15 (1977) 1111. 25

[94] A. Kennedy, I. Horváth, and S. Sint, "A New exact method for dynamical fermion computations with nonlocal actions," Nucl. Phys. Proc. Suppl. $\mathbf{7 3}$ (1999) 834-836, arXiv:hep-lat/9809092 [hep-lat]. 26

[95] M. Clark, "The Rational Hybrid Monte Carlo Algorithm," PoS LAT2006 (2006) 004, arXiv:hep-lat/0610048 [hep-lat]. 26

[96] S. Duane, A. Kennedy, B. J. Pendleton, and D. Roweth, "Hybrid Monte Carlo," Phys. Lett. B195 no. 2, (1987) 216 - 222. 26

[97] R. Scalettar, D. Scalapino, and R. Sugar, "New algorithm for the numerical simulation of fermions," Phys. Rev. B34 (1986) 7911-7917.

[98] S. A. Gottlieb, W. Liu, D. Toussaint, R. Renken, and R. Sugar, "Hybrid Molecular Dynamics Algorithms for the Numerical Simulation of Quantum Chromodynamics," Phys. Rev. D35 (1987) 2531-2542. 26

[99] G. Parisi and Y.-s. Wu, "Perturbation Theory Without Gauge Fixing," Sci. Sin. 24 (1981) 483. 26

[100] A. Ukawa and M. Fukugita, "Langevin simulation including dynamical quark loops," Phys. Rev. Lett. 55 (1985) 1854.

[101] G. Batrouni, G. Katz, A. S. Kronfeld, G. Lepage, B. Svetitsky, et al., "Langevin Simulations of Lattice Field Theories," Phys. Rev. D32 (1985) 2736. 26

[102] D. J. Callaway and A. Rahman, "The microcanonical ensemble: a new formulation of lattice gauge theory," Phys. Rev. Lett. 49 (1982) 613. 26

[103] D. J. Callaway and A. Rahman, "Lattice gauge theory in microcanonical ensemble," Phys. Rev. D28 (1983) 1506. 26 


\section{REFERENCES}

[104] N. Metropolis, A. Rosenbluth, M. Rosenbluth, A. Teller, and E. Teller, "Equation of state calculations by fast computing machines," J. Chem. Phys. 21 (1953) 1087-1092. 26

[105] E. Y. Remez, General computational methods of Chebyshev approximation: the problems with linear real parameters. ERDA Div. Phys. Res., Washington, DC, 1962. 27

[106] S.-J. Dong and K.-F. Liu, "Stochastic estimation with Z(2) noise," Phys. Lett. B328 (1994) 130-136, arXiv:hep-lat/9308015 [hep-lat]. 30

[107] TXL Collaboration, J. Viehoff et al., "Improving stochastic estimator techniques for disconnected diagrams," Nucl. Phys. Proc. Suppl. 63 (1998) 269-271, arXiv:hep-lat/9710050 [hep-lat].

[108] P. Boyle, A. Juttner, C. Kelly, and R. Kenway, "Use of stochastic sources for the lattice determination of light quark physics," JHEP 0808 (2008) 086, arXiv:0804.1501 [hep-lat]. 30

[109] G. S. Bali, S. Collins, and A. Schafer, "Effective noise reduction techniques for disconnected loops in Lattice QCD," Comput. Phys. Commun. 181 (2010) 1570-1583, arXiv:0910.3970 [hep-lat]. 33

[110] J. Foley, K. Jimmy Juge, A. O'Cais, M. Peardon, S. M. Ryan, et al., "Practical all-to-all propagators for lattice QCD," Comput. Phys. Commun. 172 (2005) 145-162, arXiv:hep-lat/0505023 [hep-lat]. 33

[111] S. Collins, G. Bali, and A. Schafer, "Disconnected contributions to hadronic structure: a new method for stochastic noise reduction," PoS LAT2007 (2007) 141, arXiv:0709.3217 [hep-lat]. 33

[112] APE Collaboration, M. Albanese et al., "Glueball masses and the loop loop correlation functions," Phys. Lett. B197 (1987) 400. 34

[113] APE Collaboration, M. Albanese et al., "Glueball Masses and String Tension in Lattice QCD," Phys. Lett. B192 (1987) 163-169. 34 
[114] B. Lucini, M. Teper, and U. Wenger, "Glueballs and k-strings in $\mathrm{SU}(N)$ gauge theories: Calculations with improved operators," JHEP 0406 (2004) 012, arXiv:hep-lat/0404008 [hep-lat]. 34

[115] B. Lucini, "The Large N limit from the lattice," Few Body Syst. 36 (2005) 161-166, arXiv:hep-ph/0410016 [hep-ph]. 34

[116] M. Teper, "The scalar and tensor glueball masses in lattice gauge theory," Phys. Lett. B185 (1987) 121. 34

[117] M. Teper, "An Improved Method for Lattice Glueball Calculations," Phys. Lett. B183 (1987) 345. 34

[118] A. Patella, "A precise determination of the psibar-psi anomalous dimension in conformal gauge theories," Phys. Rev. D86 (2012) 025006, arXiv:1204.4432 [hep-lat]. 35

[119] L. Giusti and M. Lüscher, "Chiral symmetry breaking and the Banks-Casher relation in lattice QCD with Wilson quarks," JHEP 0903 (2009) 013, arXiv:0812.3638 [hep-lat]. 35, 36

[120] B. Bolder, T. Struckmann, G. S. Bali, N. Eicker, T. Lippert, et al., "A High precision study of the $\mathrm{Q}$ anti-Q potential from Wilson loops in the regime of string breaking," Phys. Rev. D63 (2001) 074504, arXiv:hep-lat/0005018 [hep-lat]. 39

[121] A. Hasenfratz and F. Knechtli, "Flavor symmetry and the static potential with hypercubic blocking," Phys.Rev. D64 (2001) 034504, arXiv:hep-lat/0103029 [hep-lat]. 39

[122] B. Efron, "Nonparametric estimates of standard error: The jackknife, the bootstrap and other methods," Biometrika 68 no. 3, (1981) 589-599. 40

[123] P. Diaconis and B. Efron, "Computer-intensive methods in statistics," Scientific American 249 (1983) 116-130.

[124] B. Efron and R. J. Tibshirani, An Introduction to the Bootstrap. Chapman \& Hall, New York, 1993. 40 


\section{REFERENCES}

[125] R. S. Van de Water, "The CKM matrix and flavor physics from lattice QCD," PoS LAT2009 (2009) 014, arXiv:0911.3127 [hep-lat]. 42

[126] Z. Fodor, K. Holland, J. Kuti, D. Nogradi, and C. Schroeder, "Chiral symmetry breaking in nearly conformal gauge theories," PoS LAT2009 (2009) 055, arXiv:0911.2463 [hep-lat]. 44

[127] A. Hasenfratz, "Investigating the critical properties of beyond-QCD theories using Monte Carlo Renormalization Group matching," Phys. Rev. D80 (2009) 034505, arXiv:0907.0919 [hep-lat]. 44

[128] K. Miura, M. P. Lombardo, and E. Pallante, "Thermodynamic Study for Conformal Phase in Large $N_{\mathrm{f}}$ Gauge Theory," PoS LATTICE2011 (2011) 207, arXiv:1111.1098 [hep-lat].

[129] LatKMI Collaboration, Y. Aoki et al., "Many flavor QCD as exploration of the walking behavior with the approximate IR fixed point," PoS LATTICE2011 (2011) 080, arXiv:1202.4712 [hep-lat].

[130] X.-Y. Jin and R. D. Mawhinney, "Lattice QCD with 8 and 12 degenerate quark flavors," PoS LAT2009 (2009) 049, arXiv:0910.3216 [hep-lat]. 44

[131] X.-Y. Jin and R. D. Mawhinney, "Evidence for a First Order, Finite Temperature Phase Transition in 8 Flavor QCD," PoS LATTICE2010 (2010) 055, arXiv: 1011.1511 [hep-lat].

[132] A. Deuzeman, M. P. Lombardo, and E. Pallante, "The Physics of eight flavours," Phys. Lett. B670 (2008) 41-48, arXiv:0804.2905 [hep-lat]. 44

[133] P. Damgaard, U. M. Heller, A. Krasnitz, and P. Olesen, "On lattice QCD with many flavors," Phys. Lett. B400 (1997) 169-175, arXiv:hep-lat/9701008 [hep-lat]. 44

[134] U. M. Heller, "The Schrodinger functional running coupling with staggered fermions and its application to many flavor QCD," Nucl. Phys. Proc. Suppl. 63 (1998) 248-250, arXiv:hep-lat/9709159 [hep-lat]. 
[135] A. Hasenfratz, "Scaling properties of many-fermion systems from MCRG studies," PoS LAT2009 (2009) 052, arXiv:0911.0646 [hep-lat]. 44

[136] LatKMI Collaboration, Y. Aoki et al., "Many flavor QCD with $N_{f}=12$ and 16," PoS LATTICE2011 (2011) 053, arXiv:1202.4916 [hep-lat]. 44

[137] T. Appelquist, G. Fleming, M. Lin, E. Neil, and D. Schaich, "Lattice Simulations and Infrared Conformality," Phys. Rev. D84 (2011) 054501, arXiv:1106.2148 [hep-lat]. 44

[138] K. Ogawa, T. Aoyama, H. Ikeda, E. Itou, M. Kurachi, et al., "The Infrared behavior of $\mathrm{SU}(3) N_{\mathrm{f}}=12$ gauge theory -about the existence of conformal fixed point-," PoS LATTICE2011 (2011) 081, arXiv:1111.1575 [hep-lat]. 44

[139] T. Karavirta, J. Rantaharju, K. Rummukainen, and K. Tuominen, "Determining the conformal window: SU(2) gauge theory with $N_{\mathrm{f}}=4,6$ and 10 fermion flavours," JHEP 1205 (2012) 003, arXiv:1111.4104 [hep-lat]. 44

[140] T. Karavirta, J. Rantaharju, K. Rummukainen, and K. Tuominen, "Exploring the conformal window: SU(2) gauge theory on the lattice," PoS LATTICE2011 (2011) 067, arXiv:1201.2037 [hep-lat]. 44

[141] R. Lewis, C. Pica, and F. Sannino, "Light Asymmetric Dark Matter on the Lattice: SU(2) Technicolor with Two Fundamental Flavors," Phys. Rev. D85 (2012) 014504, arXiv:1109.3513 [hep-ph]. 44

[142] LSD Collaboration, G. Voronov, "Lattice study of the extent of the conformal window in two-color Yang-Mills theory," PoS LATTICE2011 (2011) 093, arXiv: 1301.4141 .44

[143] F. Bursa, L. Del Debbio, L. Keegan, C. Pica, and T. Pickup, "Mass anomalous dimension and running of the coupling in $\mathrm{SU}(2)$ with six fundamental fermions," PoS LATTICE2010 (2010) 070, arXiv:1010.0901 [hep-ph].

[144] F. Bursa, L. Del Debbio, L. Keegan, C. Pica, and T. Pickup, "Mass anomalous dimension in SU(2) with six fundamental fermions," Phys. Lett. B696 (2011) 374-379, arXiv:1007.3067 [hep-ph]. 44 


\section{REFERENCES}

[145] T. DeGrand, Y. Shamir, and B. Svetitsky, "Phase structure of SU(3) gauge theory with two flavors of symmetric-representation fermions," Phys. Rev. D79 (2009) 034501, arXiv:0812.1427 [hep-lat]. 44

[146] T. DeGrand, Y. Shamir, and B. Svetitsky, "Running coupling and mass anomalous dimension of $\mathrm{SU}(3)$ gauge theory with two flavors of symmetric-representation fermions," Phys. Rev. D82 (2010) 054503, arXiv:1006.0707 [hep-lat].

[147] T. DeGrand, Y. Shamir, and B. Svetitsky, "Gauge theories with fermions in the two-index symmetric representation," PoS LATTICE2011 (2011) 060, arXiv:1110.6845 [hep-lat]. 44

[148] T. DeGrand, Y. Shamir, and B. Svetitsky, "Mass anomalous dimension in sextet QCD," Phys. Rev. D87 no. 074507, (2013), arXiv:1201.0935 [hep-lat].

[149] Z. Fodor, K. Holland, J. Kuti, D. Nogradi, and C. Schroeder, "Chiral symmetry breaking in fundamental and sextet fermion representations of SU(3) color," arXiv:1103.5998 [hep-lat].

[150] Z. Fodor, K. Holland, J. Kuti, D. Nogradi, C. Schroeder, et al., "The sextet gauge model, light Higgs, and the dilaton," PoS LATTICE2012 (2012) 024, arXiv:1211.6164 [hep-lat].

[151] D. Sinclair and J. Kogut, "QCD thermodynamics with colour-sextet quarks," PoS LAT2009 (2009) 184, arXiv:0909. 2019 [hep-lat].

[152] D. Sinclair and J. Kogut, "New results with colour-sextet quarks," PoS LATTICE2010 (2010) 071, arXiv:1008.2468 [hep-lat].

[153] D. Sinclair and J. Kogut, "QCD with colour-sextet quarks," PoS LATTICE2012 (2012) 026, arXiv:1211.0712 [hep-lat]. 44

[154] T. DeGrand, Y. Shamir, and B. Svetitsky, "SU(4) lattice gauge theory with decuplet fermions: Schrodinger functional analysis," Phys. Rev. D85 (2012) 074506, arXiv:1202.2675 [hep-lat]. 44 
[155] Y. Aoki, T. Aoyama, M. Kurachi, T. Maskawa, K.-i. Nagai, et al., "Walking signals in $N_{\mathrm{f}}=8 \mathrm{QCD}$ on the lattice," arXiv:1302.6859 [hep-lat]. 44

[156] L. Del Debbio, B. Lucini, A. Patella, C. Pica, and A. Rago, "Conformal versus confining scenario in SU(2) with adjoint fermions," Phys. Rev. D80 (2009) 074507, arXiv:0907.3896 [hep-lat]. 46, 96

[157] F. Bursa, L. Del Debbio, D. Henty, E. Kerrane, B. Lucini, A. Patella, C. Pica, T. Pickup, and A. Rago, "Improved lattice spectroscopy of Minimal Walking Technicolor," Phys. Rev. D84 (Aug, 2011) 034506, arXiv:1104.4301. 46, 47, 96

[158] G. Bergner, T. Berheide, G. Munster, U. D. Ozugurel, D. Sandbrink, et al., "The gluino-glue particle and finite size effects in supersymmetric Yang-Mills theory," JHEP 1209 (2012) 108, arXiv:1206.2341 [hep-lat]. 64

[159] K. Levenberg, "A method for the solution of certain non-linear problems in least squares," Q. J. Appl. Math. II no. 2, (1944) 164-168. 70

[160] D. Marquardt, "An algorithm for least-squares estimation of nonlinear parameters," J. Soc. Ind. Appl. Math. 11 no. 2, (1963) 431-441. 70

[161] S. Kirkpatrick, C. D. Gelatt, and M. P. Vecchi, "Optimization by simulated annealing," Science $\mathbf{2 2 0}$ no. 4598, (1983) 671-680. 72

[162] V. Černý, "Thermodynamical approach to the traveling salesman problem: An efficient simulation algorithm," J. Optimiz. Theory App. 45 no. 1, (Jan., 1985) 41-51. 72

[163] C. G. Callan, R. Dashen, and D. J. Gross, "Toward a theory of the strong interactions," Phys. Rev. D17 (May, 1978) 2717-2763. 76

[164] A. Belavin, A. M. Polyakov, A. Schwartz, and Y. Tyupkin, "Pseudoparticle Solutions of the Yang-Mills Equations," Phys. Lett. B59 (1975) 85-87. 77

[165] S. Coleman, Aspects of Symmetry: Selected Erice Lectures. Cambridge University Press, 1988.

http://books.google.co.uk/books?id=PX2A18LE9FkC. 78 


\section{REFERENCES}

[166] S.-i. Nam, "QCD magnetic susceptibility at finite temperature beyond the chiral limit," arXiv:1304.1265 [hep-ph]. 78

[167] S.-i. Nam, "Electrical conductivity of quark matter at finite T under external magnetic field," Phys. Rev. D86 (2012) 033014, arXiv:1207.3172 [hep-ph]. 78

[168] UKQCD Collaboration, D. A. Smith and M. J. Teper, "Topological structure of the SU(3) vacuum," Phys. Rev. D58 (May, 1998) 014505, arXiv:hep-lat/9801008. 78

[169] M. Teper, "Instantons in the Quantized SU(2) Vacuum: A Lattice Monte Carlo Investigation," Phys. Lett. B162 (1985) 357. 79

[170] F. Bruckmann, F. Gruber, K. Jansen, M. Marinkovic, C. Urbach, et al., "Comparing topological charge definitions using topology fixing actions," Eur. Phys. J. A43 (2010) 303-311, arXiv:0905.2849 [hep-lat]. 79

[171] M. García Pérez, T. G. Kovács, and P. van Baal, "Comments on the instanton size distribution," Phys. Lett. B472 (2000) 295-301, arXiv:hep-ph/9911485 [hep-ph]. 84

[172] C. Michael and P. Spencer, "Cooling and the SU(2) instanton vacuum," Phys. Rev. D52 (1995) 4691-4699, arXiv:hep-lat/9503018 [hep-lat].

[173] S. Hands and P. Kenny, "Topological fluctuations in dense matter with two colors," Phys. Lett. B701 (2011) 373-377, arXiv:1104.0522 [hep-lat]. 84

[174] H. Childs, E. S. Brugger, K. S. Bonnell, J. S. Meredith, M. Miller, B. J. Whitlock, and N. Max, "A contract-based system for large data visualization," in Proceedings of IEEE Visualization 2005, pp. 190-198. 2005. 85, 121

[175] "Visit visualization tool." https://wci.llnl.gov/codes/visit/. 85, 121

[176] "What if? - FedEx bandwidth," January, 2013.

http://what-if.xkcd.com/31/. 95 
[177] L. Del Debbio, A. Patella, and C. Pica, "Fermions in higher representations. Some results about SU(2) with adjoint fermions," PoS LATTICE2008 (2008) 064, arXiv:0812.0570 [hep-lat]. 96

[178] L. Del Debbio, A. Patella, and C. Pica, "Higher representations on the lattice: Numerical simulations. SU(2) with adjoint fermions," Phys. Rev. D81 (2010) 094503, arXiv:0805.2058 [hep-lat]. 96

[179] A. Armoni, B. Lucini, A. Patella, and C. Pica, "Orientifold Planar Equivalence: The Chiral Condensate," PoS LATTICE2008 (2008) 054, arXiv:0809.5015 [hep-lat].

[180] B. Lucini, G. Moraitis, A. Patella, and A. Rago, "Orientifold Planar Equivalence: The Quenched Meson Spectrum," PoS LATTICE2010 (2010) 063, arXiv:1010.6053 [hep-lat].

[181] A. Patella, L. Del Debbio, B. Lucini, C. Pica, and A. Rago, "Confining vs. conformal scenario for SU(2) with adjoint fermions. Gluonic observables," PoS LATTICE2010 (2010) 068, arXiv:1011.0864 [hep-lat].

[182] S. Catterall, L. Del Debbio, J. Giedt, and L. Keegan, "Systematic Errors of the MCRG Method," PoS LATTICE2011 (2011) 068, arXiv:1110.1660 [hep-ph].

[183] G. Moraitis, "Spectrum of orientifold QCD in the strong coupling and hopping expansion approximation," Phys. Lett. B690 no. 1, (2010) 96 - 101, arXiv:0911.5111.

[184] R. Lewis, C. Pica, and F. Sannino, "Light asymmetric dark matter on the lattice: SU(2) technicolor with two fundamental flavors," Phys. Rev. D85 (Jan, 2012) 014504, arXiv:1109.3513.

[185] S. Catterall, L. Del Debbio, J. Giedt, and L. Keegan, "Monte Carlo Renormalization Group Minimal Walking Technicolor," Phys. Rev. D85 (May, 2012) 094501.

[186] S. Catterall, L. Del Debbio, J. Giedt, and L. Keegan, "MCRG Minimal Walking Technicolor," PoS LATTICE2010 (2010) 057, arXiv:1010.5909 [hep-ph]. 


\section{REFERENCES}

[187] A. Patella, "A precise determination of the $\bar{\psi} \psi$ anomalous dimension in conformal gauge theories," Phys. Rev. D86 (Jul, 2012) 025006, arXiv: 1204.4432 .96

[188] "International Supercomputing Conference - ISC." http://isc-events.com/isc12/. 97

[189] "The LINPACK benchmark." http://www.top500.org/project/linpack. 98

[190] "TOP500 supercomputer sites." http://www.top500.org/. 98

[191] P. Luszczek, J. J. Dongarra, D. Koester, R. Rabenseifner, B. Lucas, J. Kepner, J. Mccalpin, D. Bailey, and D. Takahashi, "Introduction to the HPC Challenge Benchmark Suite," tech. rep., 2005. 98

[192] M. Tolentino and K. W. Cameron, "The optimist, the pessimist, and the global race to exascale in 20 megawatts," Computer 45 no. 1, (Jan., 2012) 95-97. 98

[193] "The Graph 500 list." http://www.graph500.org/. 98

[194] X.-Q. Luo, E. Gregory, H. Xi, J. Yang, Y. Wang, et al., "High performance Beowulf computer for lattice QCD," Nucl. Phys. Proc. Suppl. 106 (2002) 1046-1048, arXiv:hep-lat/0202007 [hep-lat]. 98

[195] M. Lüscher, "Lattice QCD on PCs?," Nucl. Phys. B Proc. Suppl. 106-107 no. 0, (2002) $21-28$, arXiv:hep-lat/0110007. LATTICE 2001 Proceedings of the XIXth International Symposium on Lattice Field Theory. 98, 100

[196] D. J. Lilja, Measuring Computer Performance: A practitioner's guide. Cambridge, 2005. 98 